\title{
Measurement of the Survival Probability and Determination of the Three-Flavor Neutrino Oscillation Parameters at the Sudbury Neutrino Observatory
}

by

Olivier Simard, M. Sc.

\author{
A thesis submitted to the \\ Faculty of Graduate Studies and Research \\ in partial fulfillment of the requirements \\ for the degree of \\ Doctor of Philosophy \\ Ottawa-Carleton Institute for Physics \\ Department of Physics \\ Carleton University \\ Ottawa, Ontario, Canada \\ September, 2009 \\ (C)copyright \\ 2009, Olivier Simard
}




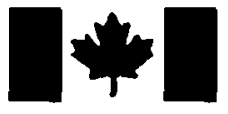

Library and Archives

Canada

Published Heritage

Branch

395 Wellington Street

Ottawa ON K1A ON4

Canada
Bibliotheque et

Archives Canada

Direction du

Patrimoine de l'édition

395, rue Wellington

Ottawa ON K1A ON4

Canada
Your file Votre référence
ISBN: 978-0-494-60122-8
Our file Notre référence
ISBN: 978-0-494-60122-8
NOTICE:

The author has granted a nonexclusive license allowing Library and Archives Canada to reproduce, publish, archive, preserve, conserve, communicate to the public by telecommunication or on the Internet, loan, distribute and sell theses worldwide, for commercial or noncommercial purposes, in microform, paper, electronic and/or any other formats.

The author retains copyright ownership and moral rights in this thesis. Neither the thesis nor substantial extracts from it may be printed or otherwise reproduced without the author's permission.
AVIS:

L'auteur a accordé une licence non exclusive permettant à la Bibliothèque et Archives Canada de reproduire, publier, archiver, sauvegarder, conserver, transmettre au public par télécommunication ou par l'Internet, prêter, distribuer et vendre des thèses partout dans le monde, à des fins commerciales ou autres, sur support microforme, papier, électronique et/ou autres formats.

L'auteur conserve la propriété du droit d'auteur et des droits moraux qui protège cette thèse. Ni la thèse ni des extraits substantiels de celle-ci ne doivent être imprimés ou autrement reproduits sans son autorisation.
In compliance with the Canadian Privacy Act some supporting forms may have been removed from this thesis.

While these forms may be included in the document page count, their removal does not represent any loss of content from the thesis.
Conformément à la loi canadienne sur la protection de la vie privée, quelques formulaires secondaires ont été enlevés de cette thèse.

Bien que ces formulaires aient inclus dans la pagination, il n'y aura aucun contenu manquant. 
Find out what it is in life that you don't do well, and then don't do that thing.

Stay thirsty my friends.

- The most interesting man in the world 


\section{Abstract}

The Sudbury Neutrino Observatory (SNO) has the ability to measure the total and electronic components of the solar neutrino flux, simultaneously, employing independent and complementary techniques. This thesis first introduces the physics of solar neutrinos as well as the mechanism of neutrino oscillation via mixing in the leptonic sector, and possible extensions to the standard model of particle physics. Then the SNO detector is described with a detailed summary of the optical calibration methods. In particular, the extraction of the relative efficiencies of SNO's photomultiplier tubes is relevant towards improvements of the last phase of the SNO experiment, and in the new low energy threshold analysis of the SNO data. The optical calibration in the last phase of the SNO experiment has shown that the detector optics had not been altered compared to previous phases, despite the major changes introduced by the insertion of an array of proportional counters to detect neutrons in SNO.

The low energy threshold analysis, that is built on numerous improvements of calibration, simulation, and analysis methods, is then introduced. It leads to the extraction of solar neutrino fluxes, but more importantly, to the measurement of the survival probability and the determination of the oscillation parameters relevant to solar neutrinos. The interpretation of the flux and spectrum results into survival probabilities are obtained from the combined low energy threshold analysis of the data of the pure heavy water and salt phases of the experiment. Especially relevant to our understanding of the neutrinos and the Sun, the survival probability is extracted with two independent parameterizations as a function of neutrino energy. Finally, a novel 
three-flavor analysis of matter-enhanced oscillation is performed leading to additional information on the neutrino mixing angle $\theta_{13}$ that has never been directly investigated with solar neutrino data. 


\section{Acknowledgements}

Encore une fois merci à ma famille et tous ceux qui m'ont accompagné durant

les cinq dernières années. Vous savez qui vous êtes et d'où vous venez. Oui, j'étais seulement venu pour faire mes études ici, mais en suis retourné avec un incroyable lot de nouvelles connaissances, d'ami(e)s, d'expériences, d'histoires, et j'en passe... sans l'ombre d'un doute j'ai beaucoup grandi. Et ça continue.

Aussi un grand merci à mes potes de bureau, Etienne Rollin et Pierre-Luc Drouin, et au plus vieux, Alain. Merci d'avoir su tolérer mes sauts d'humeurs, mes commentaires désobligeants, mes critiques saignantes, et mes innombrables blagues de routine auxquelles vous avez peut-être fini par prendre goût. Votre persévérance, intelligence et calme m'ont beaucoup aidé à travers les années. Espérons que nos chemins se recroiseront dans le futur!

Thanks to my supervisor Alain Bellerive for his advice, patience, devotion, and sense of humor. The French guys have accomplished great things within SNO after all. At Carleton, the SNO group has continuously provided great ideas, positive feedback, and shared the French-English translations of the weirdest expressions one could come up with. Thanks to Richard Hemingway who has believed in me and approached difficult situations with humor but honesty. To Peter Watson who has brought a lot of exotic ideas in the discussions. To Gordana Tešić who has traded her knowledge in theory against some computing tips. Tikish-pish, glopod! Many 
thanks to our past and current post-docs for their precious help: David Waller, Khalil Boudjemline, Feng Zhang, and Laurel Sinclair.

Among the department, thanks to all the students that have been around. I enjoyed fighting for spots in the Unicentre, having lunch at the river, and the discussions over beer and samosas at Mike's place. Thanks to John-Paul Archambault, who used to be in Alberta when I met him at TRIUMF, and who was shipped back to us in Ottawa. Let's play some banjo while we are watching people in the lobby at the next Christmas party. I'll bring the eggplant. Thanks to the older guys who have left already: Malachi and Claudiu. Many thanks to the EXO people and others: Chad, James, Russel, Deanna and Christina. Wow, you guys can drink! I also enjoyed the company of Kathleen, Ossama, Ryuichi, Thomas, Ken and Adam. Best of luck in finding the Higgs. Many thanks also to Dave Rogers and Gerald Oakham for their assistance in computing matters. These thanks of course extend to the staff at Carleton, Bill, Wade, and Dag. As many have said before me, our work would not have been possible without your support. Turn on the A/C we have some work to do!

Within the SNO Collaboration, many thanks to the LETA WG: Ryan MacLellan, Mark Kos, Stan Seibert, Gabriel Orebi-Gann, Joshua Klein and Monica Dunford. I enjoyed your great energy on phone calls, your ease of understanding complex problems, and your ability to do work in a lobby, airport, or hotel room. I also had good times with Jeff Secrest, Bill Heintzelman, Alan Poon, Jacques Farine, Ryan Martin, Chris Howard, and many others... Also big thanks to the calibration crew, Peter Skensved, José Maneira, Nuno Barros, and the Kraus(s)'s. There was for sure lots of data and obstacles, and we were successful in figuring it out. I wish you all the best in the future. More thanks to the people at Queen's, who always been very supportive 
of their Carleton friends. Special thanks to our great leader, Art McDonald. Thanks to Mark Chen for his advice on physics matters and to Aksel Hallin for his knowledge on SNO. Thanks also to the newer people on SNO, Nikolai Tolich and Jason Detwiler. In Japan, arigato to Masayuki Nakahata who has taken time off his busy schedule to introduce me to everybody at Kamioka and has shown me everything I needed to see in the laboratories. Thanks also to the boss Yoichiro Suzuki, and the extremely experienced staff Moriyama-san, Obayashi-san, and Hayato-san. Thanks to my Honda-fellows and hard-working colleges, Kota Ueshima, Takashi Iida, and Motoyasu Ikeda. Time has gone so wonderfully fast, but I enjoyed hanging out with you, trying to reverse-translate your stories about girls.

In Portugal, obrigado to José as well as the unlucky Nuno (pronounced Nonoo). Thank you guys for the great teamwork, for your warm welcome when I was in Lisbon, and for the great steaks when you were in Canada.

Many thanks to Kevin Graham, who has an unbelievable life, his wife Angela, and their blond kids. Bonk beds, inflatable mattress, couch, I lost my keys, and B\&M's house... you have entertained me so much. Thanks for your support and the faith you have put in me though. Thanks also for the endless discussions, most of them being on random subjects such as scotch, sushis, bad temper, and coat hangers.

Finally my finest appreciation to Louise Heelan, who has been a source of infinite support and who contained me whenever I was ready to burst out. There are so many things we have not done, which we should have done, because we had too many things to get done... the life of a physicist. I sincerely hope we will get to do them one day. Love you girl!

Olivier Simard, Ottawa, July 2009 


\section{Statement of Originality}

The work presented in this thesis is the author's own and is original except where noted. All use of work other than the author's and references to additional information are cited in the text. Here follows a list of accomplishments from the author's work that contributed to the success of the hereby thesis and of the Sudbury Neutrino Observatory (SNO) experiment:

SNO was designed and constructed before the author joined the Collaboration. The author has contributed to data collection and analysis since the beginning of the salt phase.

Chapters 6 and 7 reflect the main contribution of the author towards the forthcoming and final SNO neutrino physics publications. The survival probability fit is an idea that the SNO Collaboration has spoken about for many years. The author's original idea was to implement an independent framework that could not only handle the SNO data but also data from other experiments. The global fits of the survival probability has been performed for the first time by the author. The analysis of the neutrino oscillation parameters was developed and performed by the author, with precious help from Mark Chen, Ryan Martin, and Gordana Tešić. The first extraction of the mixing angle $\theta_{13}$ by SNO was performed by the author.

The code that performs the numerical integration of systems of coupled differential equations to obtain the neutrino survival probability was inspired from the Numerical Recipes [106] but adapted for precision and performance purposes partly by the author. The survival probability formulae were obtained independently by 
the author based on standard techniques that can be found in the neutrino physics literature. The code that performs the survival probability fits and global fits of neutrino oscillation parameters was written by the author in $\mathrm{C}++$ using the ROOT [102] libraries, and integrated into the SNO code package named qphysics. The latter code is independent from previous codes employed by the Collaboration to perform similar analysis.

The low energy threshold analysis, although the author's participation was exhaustive, was mainly developed by Josh R. Klein, Monica Dunford, Gabriel Orebi-Gann and Stan Seibert, with substantial contributions from many others. The data selection rules, corrections, assignment of the systematic uncertainties were set for the official analysis by the above people and used by the author to get the results presented in this thesis. The signal extraction package used in this thesis was one of the officially-approved codes by the SNO Collaboration, developed by Pierre-Luc Drouin at Carleton University. The configuration files were arranged to perform the analysis specific to this thesis, mainly with the day/night separation and variable energy bin widths.

The optical constants in the commissioning and NCD phases of the SNO experiment were extracted by the author. The optical constants in the $\mathrm{D}_{2} \mathrm{O}$ and salt phases had been extracted in the past by Bryce A. Moffat, Darren R. Grant, and Ranpal S. Dosanjh, but were re-extracted by the author after several improvements were made to the analysis framework by the author and other collaborators. Hence the author has handled the entire SNO experiment optical calibration data set and has determined the optical constants of the detector for the three phases of the experiment.

The data collected during optical calibration runs were the result of the collective 
work of the SNO calibration group. The improvements in the calibration analyses were jointly implemented by the author, based on work that had been previously done for previous phases of SNO, with the help of José C. Maneira, Aksel Hallin, Peter Skensved, Christine Kraus, and Nuno F. Barros. The new concepts developed for the NCD phase such as the NCD position fit method and reflectivity geometrical corrections were the original ideas of the author.

This thesis was typeset using $\operatorname{taT}_{\mathrm{E}} \mathrm{X} 2 \varepsilon$ by the author. Figure 1.3, page 13, was made using Jaxodraw [157]. Figure 4.4b, page 94, was made using XSnoed, the SNO event display software [158]. All other figures are the author's original creation, with the help of the ROOT graphical libraries [102].

The author has no responsibility for the persistence or accuracy of URLs for external or third-party internet websites referred to in this thesis, and does not guarantee that any content on such websites is, or will remain, accurate or appropriate. 


\section{Contents}

Abstract $\quad$ iii

$\begin{array}{ll}\text { Acknowledgements } & \text { v }\end{array}$

Statement of Originality viii

Contents $\quad$ xi

List of Tables $\quad$ xvi

List of Figures $\quad$ xviii

List of Abbreviations and Symbols xxii

1 Introduction 1

1.1 Standard Model of Particle Physics . . . . . . . . . . . . . . 3

1.2 Astrophysics and Neutrino Physics . . . . . . . . . . . . 5

1.2.1 Solar Standard Model . . . . . . . . . . . . 6

1.2.2 Neutrino Oscillation . . . . . . . . . . . . 9

1.2.3 Dual Search with Solar Neutrinos . . . . . . . . . . 15

1.3 Solar Neutrino Experiments . . . . . . . . . . . . . . 16

1.3.1 First-Generation Experiments . . . . . . . . . . . 16

1.3.2 Second-Generation Experiments . . . . . . . . . . 18

1.3.3 SNO and the Future . . . . . . . . . . . . . 21

1.4 Synopsis . . . . . . . . . . . . . . . . . . 27

2 The SNO Detector 28

2.1 Detector Configurations . . . . . . . . . . . . . . 31

2.1.1 Pure Heavy Water Phase . . . . . . . . . . . . . . 31

2.1 .2 Salt Phase . . . . . . . . . . . . . . 32

2.1.3 Neutral Current Detector Phase . . . . . . . . . . . . 32 
2.2 PMT System . . . . . . . . . . . . . . . . . . 33

2.2 .1 Electronic and Trigger Systems _. . . . . . . . . 33

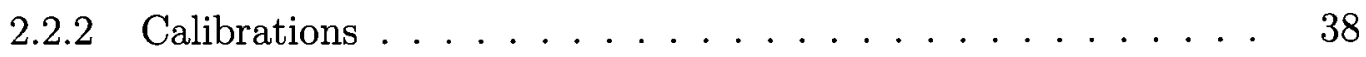

2.3 NCD System . . . . . . . . . . . . . . . . . . . 42

2.3.1 Electronic and Trigger Systems _ . . . . . . . . . . . 43

2.3 .2 Calibrations . . . . . . . . . . . . . . . . 45

2.4 Backgrounds and Water Systems $\ldots \ldots \ldots \ldots \ldots$

2.4.1 Low Energy Backgrounds . . . . . . . . . . . . . . 48

2.4.2 Background Measurement Techniques . . . . . . . . . 52

2.5 Detector Simulation . . . . . . . . . . . . . . . . . 55

3 Optical Calibration in the NCD Commissioning Phase 58

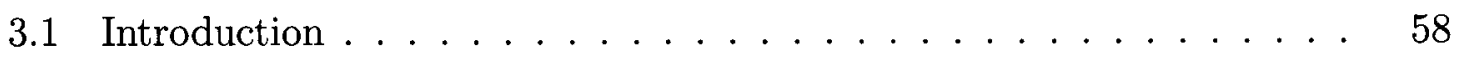

3.2 Optical Calibration Concepts $\ldots \ldots \ldots \ldots \ldots \ldots$

3.2 .1 Optical Model . . . . . . . . . . . . . . . . . . . . 61

3.2 .2 Occupancy-Ratio Method . . . . . . . . . . . 63

3.2 .3 Occupancy-Efficiency Method . . . . . . . . . . . 66

3.3 Extraction of Relative PMT Efficiencies . . . . . . . . . . . . 66

3.3.1 Normalization of Raw Efficiencies . . . . . . . . . . . 67

3.3.2 Statistical Uncertainties . . . . . . . . . . . . . . . 69

3.3 .3 PMT Variability . . . . . . . . . . . . 70

3.3.4 Local Response Variations . . . . . . . . . . . . . . . . 72

3.4 Extraction of the Optical Model Parameters . . . . . . . . . . 72

3.4.1 Results and Comparison of the Methods . . . . . . . . . . 74

3.4.2 Systematic Uncertainties due to PMT Efficiencies . . . . . . . 74

3.4 .3 Summary . . . . . . . . . . . . . . 77

4 Optical Calibration in the NCD Phase $\quad \mathbf{7 8}$

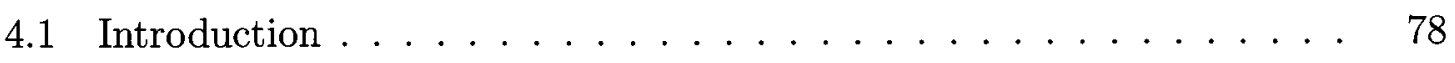

4.1.1 Optical Calibration Method and Data Sets . . . . . . . 78

4.1 .2 Overview of the Analysis . . . . . . . . . . . . 79

4.2 Laserball Source Positions . . . . . . . . . . . . . . . . . . 81

4.2 .1 PMT Times . . . . . . . . . . . . . 81 
4.2 .2 Source Position Fit . . . . . . . . . . . . . . . . . 82

4.2 .3 Uncertainties . . . . . . . . . . . . . . . . . 82

4.3 Extraction of the NCD Positions . . . . . . . . . . . . 83

4.3.1 Method of Relative Occupancy Mapping . . . . . . . . . 84

4.3 .2 Results . . . . . . . . . . . . . . . . . . 87

4.3.3 Average NCD Positions . . . . . . . . . . . . . . 91

4.4 NCD Shadows and Reflections . . . . . . . . . . . . . . . . 91

4.4 .1 NCD Shadows . . . . . . . . . . . . . . . . . . . . 92

4.4 .2 NCD Reflections . . . . . . . . . . . . . . . 94

4.4.3 Changes in the NCD Phase . . . . . . . . . . . 96

4.5 Optical Parameters for the NCD Phase . . . . . . . . . . . . 97

4.5.1 Data Set and Selection . . . . . . . . . . . . . . 97

4.5 .2 Results . . . . . . . . . . . . . . . . . 98

4.6 Uncertainties due to Optics . . . . . . . . . . . . . . . . . . 104

4.6.1 Systematic Uncertainties of the Optical Constants . . . . . . 105

4.6.2 Effects on Vertex Reconstruction . . . . . . . . . . . 106

4.6.3 Effects on Energy Estimation . . . . . . . . . . . . 107

4.7 Conclusion on Optical Calibration . . . . . . . . . . . . . . 109

5 Extraction of the SNO Signals $\quad 112$

5.1 The Low Energy Threshold Analysis . . . . . . . . . . . . . . 112

5.1.1 Observables and Combined Analysis . . . . . . . . . 113

5.1 .2 Treatment of Backgrounds . . . . . . . . . . . . . 117

5.1.3 Treatment of Systematic Uncertainties . . . . . . . . . . 118

5.2 Likelihood Fit . . . . . . . . . . . . . . . . . . . . . . . . . . 119

5.2 .1 Formalism . . . . . . . . . . . . . . . . . . 119

5.2 .2 Configuration . . . . . . . . . . . . . . . . 121

5.2.3 Verification of the Extraction Method . . . . . . . . . 126

5.3 Results . . . . . . . . . . . . . . . . . . . . . 128

$5.3 .1 \quad$ NC Flux . . . . . . . . . . . . . . . . . . . . . . . . . . . . 129

$5.3 .2 \quad \mathrm{CC}$ and ES Spectra . . . . . . . . . . . . 130

5.3.3 Background and Systematic Parameters _. . . . . . . . 131

5.3.4 Summary, but not the End . . . . . . . . . . . . . . . 134 
6 Extraction of the Survival Probability of Solar Neutrinos

6.1 From Neutrino Interactions to Observed Rates . . . . . . . . . . . 136

6.1.1 Solar Neutrino Spectrum and Survival Probability . . . . . . 137

6.1.2 Interaction Cross-Sections . . . . . . . . . . . . . 138

6.1.3 Detector Response . . . . . . . . . . . . . . . . . . . 139

6.1 .4 Analytic Convolution . . . . . . . . . . . . . . . . . 142

6.1 .5 Fractional Rates . . . . . . . . . . . . . . . . 143

6.1 .6 Figure of Merit . . . . . . . . . . . . . . . . . . . 144

6.2 Survival Probability Functions . . . . . . . . . . . . . 146

6.2 .1 Analytic Function . . . . . . . . . . . . . . . . 147

6.2 .2 Binned Function . . . . . . . . . . . . . . . . 148

6.2.3 Verification of the Extraction Method . . . . . . . . . 148

6.3 Results . . . . . . . . . . . . . . . . . . . . . . 151

6.3 .1 Results from SNO . . . . . . . . . . . . . . . . . . 151

6.3.2 Results from Solar Neutrino Experiments . . . . . . . . . 156

6.3 .3 Conservation of Unitarity . . . . . . . . . . . . . 160

6.3 .4 Summary . . . . . . . . . . . . . . 163

7 Extraction of the Neutrino Oscillation Parameters $\quad 165$

7.1 Survival Probability Formulae . . . . . . . . . . . . . . 165

7.1.1 Phenomenology of Neutrino Oscillation . . . . . . . . . 166

7.1 .2 Two-State Formula . . . . . . . . . . . . . . . . . . . 169

7.1.3 Three-State Formula . . . . . . . . . . . . . . . . . . . 169

7.2 Calculation of the Survival Probability . . . . . . . . . . . 171

7.2 .1 Propagation in the Sun . . . . . . . . . . . . . 173

7.2 .2 Propagation in the Earth . . . . . . . . . . . . 175

7.2.3 Effects of the Third Mass Eigenstate . . . . . . . . . 178

7.3 Parameter Constraints . . . . . . . . . . . . . . . . . . 182

7.3 .1 Results from SNO . . . . . . . . . . . . . . . . 183

7.3.2 Results from Solar Neutrino Experiments . . . . . . . . . . 186

7.3.3 Results from the Global Fit . . . . . . . . . . . . . . . 189

7.3 .4 Summary . . . . . . . . . . . . . . . . . . . . 194 
A More on Optical Calibration $\quad 202$

A.1 PMT Occupancy Corrections . . . . . . . . . . . . . . 202

A.1.1 Multiple Photo-Electron Correction . . . . . . . . . . . . 203

A.1.2 NCD Reflection Correction . . . . . . . . . . . . . . . 204

A.2 Systematic Uncertainties in the NCD Phase . . . . . . . . . . 208

A.2.1 Existing Systematic Errors . . . . . . . . . . . . . . . 209

A.2.2 PMT Efficiencies and Detector Response . . . . . . . . . 214

A.2.3 New Systematic Errors for the NCD Phase . . . . . . . . . 215

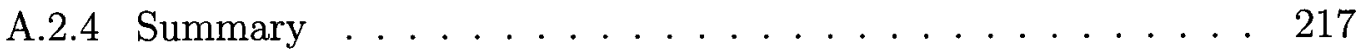

A.3 Media Attenuation Lengths $\ldots \ldots \ldots \ldots . \ldots . \ldots 218$

A.3.1 Total Extinctions . . . . . . . . . . . . . . . . . . 221

A.3.2 Pure Media Attenuation Lengths . . . . . . . . . . . . 221

A.3.3 Contributions from NCD Reflections . . . . . . . . . . . . 222

A.4 PMT Angular Response . . . . . . . . . . . . . . . . . 224

$\begin{array}{ll}\text { B Parameters in Signal Extraction } & 227\end{array}$

C More on the Survival Probability and Oscillation Parameters 229

C.1 More on Survival Probabilities . . . . . . . . . . . . . . . . . 229

C.1.1 SNO Response to Neutrons . . . . . . . . . . . . . . . . 229

C.1.2 Survival Probability Binned Function . . . . . . . . . . . 230

C.1.3 Solar Neutrino Experiments . . . . . . . . . . . . . . . 233

C.2 More on Neutrino Oscillation Parameters . . . . . . . . . . . 235

C.2.1 KamLAND . . . . . . . . . . . . . . . . 236

C.2.2 SSM Partial Derivatives . . . . . . . . . . . . . 236

C.2.3 Contours from Individual Experiments . . . . . . . . . . 238

C.2.4 Recipe to Interpret Survival Probabilities . . . . . . . . . . 239

$\begin{array}{ll}\text { Bibliography } & 243\end{array}$ 


\section{List of Tables}

1.1 Solar neutrino production reactions of the proton-proton chain and carbon-nitrogen-oxygen cycle. . . . . . . . . . . . . 8

2.1 Phases of the SNO experiment. . . . . . . . . . . . . 31

2.2 Wavelengths available for optical calibrations. . . . . . . . . . 41

2.3 Main sources of backgrounds in SNO . . . . . . . . . . . . . 54

4.1 Full optical scans taken during the NCD phase. . . . . . . . . . . . 80

4.2 Average NCD positions from all optical scans. . . . . . . . . . . . 90

4.3 Statistics available for the extraction of the optical constants throughout the NCD phase. . . . . . . . . . . . . . . . . . . 99

4.4 Summary of the systematic uncertainties in the NCD phase. . . . . 106

5.1 Binning and analysis range of the signal extraction observables. . . . 124

5.2 Parameters in the signal extraction fit. . . . . . . . . . . . . 125

6.1 Analytic survival probability parameters extracted from the SNO-LETA day/night analysis. . . . . . . . . . . . . . . 155

6.2 Binned survival probability parameters extracted from the SNO-LETA day/night analysis. . . . . . . . . . . . . . 155

6.3 Summary of the analytic survival probability parameters. . . . . . . 163

7.1 Limits and step sizes in the neutrino oscillation parameter scan. . . . 172

7.2 Confidence levels in one and two dimensions. . . . . . . . . . . . 183

7.3 Summary of the measurements of the neutrino oscillation parameters. 196

A.1 Systematic uncertainties on the optical constants in the NCD phase. . 209

A.2 Decomposition of the systematic errors on the media attenuations. . . 219

A.3 Decomposition of the systematic errors on the PMT angular response. 220

A.4 Heavy water inverse total extinction lengths in the NCD phase. . . . 221

A.5 Light water inverse total extinction lengths in the NCD phase. . . . . 221 
A.6 Reference values of Rayleigh scattering attenuation lengths. . . . . . . 222

A.7 Media pure inverse attenuation lengths in the NCD phase. . . . . . 223

A.8 Average absorption coefficients from NCD reflections. . . . . . . . . 223

B.1 Name and description of the parameters in the signal extraction fit. . 228

C.1 Data from other solar neutrino experiments. . . . . . . . . . . . 234

C.2 Systematic uncertainties of the SSM in the form of partial derivatives. 237 


\section{List of Figures}

1.1 Nuclear reactions involved in the proton-proton fusion chain. . . . . . 7

1.2 Neutrino flux spectra. . . . . . . . . . . . . . . . . 9

1.3 Feynman diagrams that generate the $\mathrm{CC}$ and NC potentials in matterenhanced oscillation. . . . . . . . . . . . . . 13

1.4 Confidence regions of the oscillation parameters obtained with the solar neutrino and KamLAND experiments. . . . . . . . . . . . . . 24

1.5 Ratio of experimental rates to the SSM prediction. . . . . . . . 25

2.1 Schematic view of the SNO detector and PMT specifications. . . . . . 30

2.2 The SNO geometry during the NCD phase. . . . . . . . . . . . 34

2.3 Schematic of a SNO Neutral Current Detector. . . . . . . . . . . 35

2.4 Schematics of the SNO source manipulator system and laserball source. 40

2.5 Radioactive decay chains of ${ }^{238} \mathrm{U}$ and ${ }^{232} \mathrm{Th} . \ldots \ldots$. . . . . . . 50

3.1 Distributions of relative efficiencies extracted from the October 2003 data at $500 \mathrm{~nm}$. . . . . . . . . . . . . . . 68

3.2 Correlation and relative difference between the PMT relative efficiencies extracted from the $\mathrm{D}_{2} \mathrm{O}$ scans. . . . . . . . . . . . . . . 69

3.3 Distribution of the uncertainties on the PMT efficiencies extracted from the $\mathrm{D}_{2} \mathrm{O}$ scans. . . . . . . . . . . . . . . 70

3.4 PMT variability as a function of PMT angle of incidence extracted from the $\mathrm{D}_{2} \mathrm{O}$ scans. . . . . . . . . . . . . . . . 71

3.5 PMT relative efficiencies as a function of PMT coordinates extracted from the $\mathrm{D}_{2} \mathrm{O}$ scans. . . . . . . . . . . . . . . 73

3.6 Media attenuation coefficients obtained from the $\mathrm{D}_{2} \mathrm{O}$ scans. . . . . 75

3.7 PMT relative angular response obtained from the $\mathrm{D}_{2} \mathrm{O}$ scans. . . . . 75

4.1 Mechanics of the relative occupancy mapping method. . . . . . . . 86

4.2 Selected M-series NCD positions fitted from the February 2006 scan. 88 
4.3 Selected M-series NCD tilts fitted from the February 2006 scan. . . . 89

4.4 Effect and pattern of the NCD shadow cut. . . . . . . . . . . 94

4.5 Quantities involved in the PMT occupancy correction due to NCD reflections. ........................... 95

4.6 Media inverse total extinction lengths as a function of wavelength in the NCD phase. . . . . . . . . . . . . . . . 101

4.7 PMT relative angular response in the NCD phase. . . . . . . . . . 102

4.8 Laserball relative intensity distribution and mask function in the NCD phase. ........................ 103

4.9 Media inverse total extinction lengths as a function of time in the NCD phase. . . . . . . . . . . . . . . . . 104

4.10 Effect of the new optics systematic errors on vertex reconstruction obtained with FTN. . . . . . . . . . . . . . . . . 108

4.11 Effect of the new optics systematic errors on energy scale and resolution. 109

5.1 Expected SNO signal shapes due to solar neutrinos in the low energy threshold analysis. . . . . . . . . . . . . . . 114

5.2 Mean biases and pulls of the signal extraction procedure. . . . . . . 129

5.3 Result of the signal extraction procedure. . . . . . . . . . . . . 132

5.4 Correlation coefficients between the SNO signals. . . . . . . . . . . 133

5.5 Background and systematic uncertainty parameters. . . . . . . . . 134

6.1 Cross-sections of the SNO reactions. . . . . . . . . . . . . 140

6.2 SNO electron response function. . . . . . . . . . . . . . . . . 142

6.3 Comparison of the SNO signal shapes obtained from the analytic model and MC simulation. . . . . . . . . . . . . . . 145

6.4 Mean bias and pull of survival probability fits performed on ensemble data sets. . . . . . . . . . . . . . . . 149

6.5 Mean bias of analytic survival probability fits for different orders performed on ensemble data sets. . . . . . . . . . . . . . . 151

6.6 Survival probability functions extracted from the SNO-LETA day/night analysis. . . . . . . . . . . . . . . . . 154 
6.7 Survival probability function extracted from all solar neutrino experiments. . . . . . . . . . . . . . . . . . . . 161

6.8 Survival, transition, and total probability functions extracted from all solar neutrino experiments. . . . . . . . . . . . . . . . 162

7.1 Radial profiles of the electron density and neutrino production as a function of position in the Sun. . . . . . . . . . . . . . . . . 174

7.2 Profile of the matter density inside the Earth. . . . . . . . . . 177

7.3 Experimental livetime distributions of the three SNO phases. . . . . 178

7.4 Effects of $\theta_{13}$ on the three-neutrino survival probabilities. . . . . 180

7.5 Effects of $\Delta m_{31}^{2}$ on the three-neutrino survival probabilities. . . . . 181

7.6 Confidence regions of the oscillation parameters obtained with SNOLETA in the two-dimensional space. . . . . . . . . . . . . . 185

7.7 Confidence regions of the oscillation parameters obtained with solar experiments. . . . . . . . . . . . . . . . . 187

7.8 Confidence regions of the oscillation parameters obtained with solar experiments and KamLAND. . . . . . . . . . . . . . . . . . 190

7.9 Confidence regions of the oscillation parameters obtained with the combined fit of solar and KamLAND experiments for the two-flavor model. 191

7.10 Confidence regions of the oscillation parameters obtained with the combined fit of solar and KamLAND experiments for the three-flavor model.193

7.11 Projections of the $\chi^{2}$-space onto each parameter axis in the three-flavor analysis. . . . . . . . . . . . . . . . 195

8.1 Survival probability functions extracted from solar neutrino data. . . 199

A.1 PMT quantum efficiency and angular response extrapolation. . . . . 226

A.2 PMT response surface as a function of wavelength and incident angle. 226

C.1 SNO neutron response function. . . . . . . . . . . . . . . 231

C.2 Comparison of the SNO NC signal shapes obtained from the analytic model and MC simulation. . . . . . . . . . . . . . . . 231

C.3 Effect of binning the survival probability on the expected SNO CC spectrum. . . . . . . . . . . . . . . . . 232 
C.4 Confidence regions of the oscillation parameters obtained with the lowenergy experiments. . . . . . . . . . . . . . . . . 240

C.5 Confidence regions of the oscillation parameters obtained with the lowenergy and Super-Kamiokande experiments. . . . . . . . . . 241 


\section{List of Abbreviations and Symbols}

\begin{tabular}{|c|c|}
\hline $\mathrm{ADC}$ & Analog-to-Digital Converter \\
\hline AV & Acrylic Vessel \\
\hline BG & BackGround \\
\hline $\mathrm{CC}$ & Charged Current \\
\hline CKM & Cabibbo-Kobayashi-Maskawa (mixing matrix) \\
\hline CL & Confidence Level \\
\hline $\mathrm{CPU}$ & Central Processing Unit (referred to as computing power) \\
\hline DAQ & Data AQuisition system \\
\hline DCR & Deck Clean Room \\
\hline ES & Elastic Scattering \\
\hline FEE & Front-End Electronics \\
\hline FTK & SNO event energy estimation algorithm (LETA) \\
\hline FTN & SNO event position reconstruction algorithm (NCD phase) \\
\hline FTP & SNO event position reconstruction algorithm (LETA) \\
\hline GALLEX & GALLium EXperiment \\
\hline GNO & Gallium Neutrino Observatory \\
\hline GS & Grid-Scan (method to extract oscillation parameters) \\
\hline $\mathrm{HE}$ & High Energy \\
\hline $\mathrm{IH}$ & Inverted Hierarchy (of neutrino masses) \\
\hline KamiokaNDE & Kamioka Nucleon Decay Experiment \\
\hline KamLAND & Kamioka Liquid scintillator ANtineutrino Detector \\
\hline LB & LaserBall \\
\hline LE & Low Energy \\
\hline LETA & Low Energy Threshold Analysis \\
\hline LOW & LOW (solution) \\
\hline LMA & Large Mixing Angle (solution) \\
\hline $\mathrm{MC}$ & Monte Carlo (simulation) \\
\hline MINOS & Main Injector Neutrino Oscillation Search \\
\hline
\end{tabular}




$\begin{array}{ll}\text { MN } & \text { MiNimization (method to extract oscillation parameters) } \\ \text { MNSP } & \text { Maki-Nakagawa-Sakata-Pontecorvo (mixing matrix) } \\ \text { MPE } & \text { Multi Photo-Electron (effect) } \\ \text { MSW } & \text { Mikheyev-Smirnov-Wolfenstein (effect) } \\ \text { NC } & \text { Neutral Current } \\ \text { NCD } & \text { Neutral Current (rather neutron) Detector } \\ \text { NCDR } & \text { NCD Reflection } \\ \text { NDF/ndf } & \text { Number of Degrees of Freedom } \\ \text { NH } & \text { Normal Hierarchy (of neutrino masses) } \\ \text { OCA } & \text { Optical CAlibration } \\ \text { OccRatio } & \text { Occupancy Ratio } \\ \text { OWL } & \text { OutWard Looking (PMT) } \\ \text { PDF } & \text { Probability Density Function } \\ \text { PE } & \text { Photo-Electron } \\ \text { PMT } & \text { PhotoMultiplier Tube } \\ \text { PMTR } & \text { PhotoMultiplier Tube angular Response } \\ \text { PSUP } & \text { PMT SUPport structure } \\ \text { QSNO } & \text { SNO C++ analysis package } \\ \text { RMS } & \text { Root Mean Square } \\ \text { RS } & \text { Rayleigh Scattering } \\ \text { RSP } & \text { SNO event energy estimation algorithm (NCD phase) } \\ \text { SAGE } & \text { Soviet-American Gallium Experiment } \\ \text { SigEx } & \text { Signal Extraction } \\ \text { SK } & \text { Super-KamiokaNDE } \\ \text { SM } & \text { Standard Model (of particle physics) } \\ \text { SNO } & \text { Sudbury Neutrino Observatory } \\ \text { SNOMAN } & \text { SNO Monte carlo and ANalysis program } \\ \text { SNP } & \text { Solar Neutrino Problem } \\ \text { SNU } & \text { Solar Neutrino Unit } \\ \text { SSM } & \text { Solar Standard Model } \\ \text { stat } & \text { Statistical (uncertainty) } \\ \text { syst } & \text { Systematic (uncertainty) } \\ & \\ & \\ & \\ \text { SMxiii }\end{array}$




$\begin{array}{ll}\oplus & \text { Earth } \\ \odot & \text { Sun } \\ A & \text { Number of nucleons } \\ Z & \text { Number of protons } \\ { }_{Z} \mathrm{X} & \text { Element chemical symbol } \\ X(a, b) Y & \text { Reaction of the type } a+X \rightarrow Y+b \\ \check{\mathrm{C}} & \text { Cerenkov/Cherenkov (radiation) } \\ \mathrm{MeV} & \text { Mega Electron-Volt (energy) } \\ \nu_{i} / \nu(i) & \text { Neutrino particle (from nuclear reaction } i \text { ) } \\ n \mathrm{D} & n \text {-dimensional, where } n \text { in an integer } \\ P_{e e} & \text { Survival probability (of electron neutrinos) } \\ \mathrm{d} & \text { Detector } \\ T_{\mathrm{eff}} & \text { Event effective kinetic energy } \\ \beta_{14} & \text { Event isotropy } \\ \rho & \text { Volume weighted radial event position } \\ \cos \theta_{\odot} & \text { Event direction relative to the Sun-Earth axis } \\ \theta_{j k} & \text { Mixing angle for mass eigenstates } j \text { and } k \text {. } \\ \Delta m_{k j}^{2} & \text { Squared mass difference for mass eigenstates } k \text { and } j . \\ \mathrm{Tl} & \text { Thallium } \\ \mathrm{Bi} & \text { Bismuth } \\ \mathrm{Po} & \text { Polonium } \\ \mathrm{Rn} & \text { Radon } \\ \mathrm{Th} & \text { Thorium } \\ \mathrm{U} & \text { Uranium } \\ \mathrm{PMT} & \text { Radioactivity of the form } \beta-\gamma \text { from PMT components } \\ & \end{array}$




\section{CHAPTER 1}

\section{Introduction}

The story of neutrinos $(\nu)$ starts in 1930 when Pauli first postulates the existence of a neutral particle emitted simultaneously with an electron by decaying nuclei. The neutrino was the perfect solution to explain the observed continuous energy spectrum of the electron without breaking the fundamental energy conservation rule (see Chapter 1 of [1] for a complete historical review). Since then the neutrinos have been associated with the weak interaction, which accounts for their extremely long penetration lengths in matter and complicates their detection. Nuclear fusion also proceeds via the weak interaction but, unlike fission, is a process that is hard to recreate and observe in a laboratory because of the high temperatures, pressures, and energies involved. The Sun, however, shines huge quantities of energy by the means of thermonuclear fusion and is expected to emit a large number of solar neutrinos, that, because of their interaction with matter, should be detectable on Earth.

From 1970 to 1990 solar neutrinos have been observed on Earth, establishing a new and very active research area in both astrophysics and particle physics: neutrino physics. The activity in that sector increased even more lately with the emergence of complex real-time detectors and longer data acquisition periods. The observation of solar neutrinos was a great experimental success story until the measurements of the solar neutrino fluxes on Earth were shown to disagree by many standard deviations 
with the rates predicted by solar models. The rates were determined to be between $1 / 3$ and $1 / 2$ with respect to the model expectations. This so-called solar neutrino problem (SNP) was later solved by allowing transitions between neutrino flavors. These transitions, which cause the solar neutrinos produced as electron neutrinos $\left(\nu_{e}\right)$ to change into the other two flavors $\left(\nu_{\mu}\right.$ and $\left.\nu_{\tau}\right)$, explained the observed deficits. This quantum mechanical phenomenon, called oscillation, can only occur if neutrinos are mixed, and can only be mixed if they have masses. Thus the SNP was solved and opened the door to another branch of research in particle physics: neutrino oscillation. Although some refinements are needed to understand the Sun more precisely, the neutrinos are being investigated intensively so that their type, mass, and mixing mechanism can be determined with higher accuracy.

Until today, the field of neutrino physics has been rich in hypotheses and measurements to confirm or refute theoretical models or experimental results. The future will bring more precision on what is already known about neutrinos, and perhaps even new information. This thesis, written in the context of the data taken at the Sudbury Neutrino Observatory, stands in between the experimental and theoretical aspects, and at the boundary between the discovery and the precision era in solar neutrino oscillation physics. The goal is twofold: characterize neutrino mixing, probing the fundamental theory of particle physics, and confirm the solar nuclear reaction rates predicted by models to strengthen the understanding of stellar physics. This chapter introduces the basic concepts and framework leading to the characterization of solar neutrinos. 


\subsection{Standard Model of Particle Physics}

The building blocks of nature, or elementary particles, are classified into half-integer and integer spin particles. The half-integer spin particles are called fermions, broken into quarks and leptons, and integer-spin particles are called bosons. The standard model (SM) of particle physics describes the interactions of these elementary particles through a set of gauge bosons, the force carriers, each responsible for specific types of interactions. The SM is therefore a gauge theory, formulated in the framework of quantum field theory, that explains the electromagnetic, weak, and strong interactions. The corresponding local symmetry group is $\mathrm{SU}(3)_{C} \times \mathrm{SU}(2)_{L} \times \mathrm{U}(1)_{Y}$, where $C$, $L$ and $Y$ are quantum numbers called color, chirality, and hypercharge, respectively. Unlike quarks that carry all three quantum numbers of the theory, the interactions of leptons, like neutrinos and electrons, can be described by the electroweak part of the model, $\mathrm{SU}(2)_{L} \times \mathrm{U}(1)_{Y}$, only. The mass of the fermions in the $\mathrm{SM}$ are created through the Higgs mechanism, which is the interaction of the fermions with a scalar, spinless, particle, the Higgs boson, that has not been discovered yet in experiments. The latter mechanism, though, cannot predict the masses of the particles which need to be measured experimentally. Neutrinos are chargeless and massless in the minimal SM. Hence in the context of the SM the neutrinos neither participate in electromagnetic processes nor interact with the Higgs.

The fermions are divided into sub-categories, the families or generations, usually associated with an additional quantum number, flavor, and differentiated by mass. The flavor can take three different values for leptons denoted by the letters $e, \mu$, and $\tau$. The number of flavors of neutrinos was measured by the Large Electron-Positron 
collider (LEP) experiments at the European Centre for Nuclear Research (CERN) to be $N_{\nu}=2.9840 \pm 0.0082[2]$.

In general the fermion flavor and mass eigenstates can be different from the point of view of the interactions through which observations are made. The correspondence between the flavor and mass bases is given by a unitary transformation, called mixing matrix. In the quark sector, the Cabibbo-Kobayashi-Maskawa (CKM) matrix describes the mixing of the quark mass eigenstates to the flavor eigenstates. The most popular parameterization [2] of the complex matrix depends on three mixing angles $\left(\theta_{j k}\right.$ with $j, k=1,2,3$ and $\left.j \neq k\right)$ and one phase $\delta$ :

$$
V_{\mathrm{CKM}}=\left(\begin{array}{ccc}
c_{12} c_{13} & s_{12} c_{13} & s_{13} e^{-i \delta} \\
-s_{12} c_{23}-c_{12} s_{23} s_{13} e^{i \delta} & c_{12} c_{23}-s_{12} s_{23} s_{13} e^{i \delta} & s_{23} c_{13} \\
s_{12} s_{23}-c_{12} c_{23} s_{13} e^{i \delta} & -c_{12} s_{23}-s_{12} c_{23} s_{13} e^{i \delta} & c_{23} c_{13}
\end{array}\right),
$$

where $c_{j k} \equiv \cos \theta_{j k}$ and $s_{j k} \equiv \sin \theta_{j k}$. The phase $\delta$ is the CP-violating phase, which if different from zero, breaks the symmetry of interactions involving the mixing matrix under the combined transformation of charge conjugation $(\mathrm{C})$ and parity $(\mathrm{P})$. The CP symmetry combined with time reversal $(\mathrm{T})$, or CPT, is always enforced and is a conservation law of nature in quantum field theory.

A formalism similar to the CKM matrix can be constructed for neutrinos if they are massive. This new formalism in the lepton sector is a modification of the SM, or the manifestation of new physics. The evidence for neutrino oscillation from the observation of deficits in atmospheric neutrino rates by the Super-Kamiokande Collaboration [3] was the first strong evidence for such physics beyond the SM. The neutrino mass should be inversely proportional to an unknown symmetry-breaking 
scale beyond the SM, and the smallness of neutrino mass is an early indication that the latter scale is very high in energy. Neutrinos can indirectly provide valuable information about the new physics related to those energy scales. In addition, the nature of neutrinos, whether they are their own anti-particles (Majorana) or not (Dirac), needs to be established. The very first step though is to understand neutrino oscillation and perhaps the differences between the mixing of quarks and leptons. Section $1.2 \mathrm{ex}$ plains how the solar neutrinos can undergo flavor transition and how the experimental solution to the SNP confirmed that mechanism.

\subsection{Astrophysics and Neutrino Physics}

The Sun is a star of the main sequence, at the hydrogen-burning epoch of stellar evolution, with a luminosity of $L_{\odot}=2.4 \times 10^{39} \mathrm{MeV} \mathrm{s}^{-1}$, a mass of $M_{\odot}=1.988 \times 10^{30} \mathrm{~kg}$, and a radius of $R_{\odot}=6.961 \times 10^{5} \mathrm{~km}$. The thermonuclear reactions that power the Sun produce both thermal energy (photons) and neutrinos. The thermal energy takes up to $10^{4}$ years before reaching the surface in the form of radiation; oppositely neutrinos escape the Sun in about 2 seconds. Thus neutrinos carry instantaneous and valuable information about the interior of the Sun. With an approximate neutrino luminosity of $L_{\odot}^{\nu} \simeq 0.02 L_{\odot}$, and at a distance of one astronomical unit (AU), corresponding to $149.6 \times 10^{6} \mathrm{~km}$, the expected energy flux of neutrinos at the Earth is about $\Phi^{\nu} \simeq 10^{10} \mathrm{MeV} \mathrm{cm}^{-2} \mathrm{~s}^{-1}$.

Figure 1.1 summarizes the nuclear reactions of the proton-proton (pp) fusion chain, highlighting the neutrino types that are produced in parentheses. Each type corresponds to a signature reaction. The notation ${ }_{Z}^{A} \mathrm{X}$ gives the composition of the elements, where $A$ is the number of nucleons or the mass number, $Z$ is the number of 
protons, and $\mathrm{X}$ is the chemical symbol of the element. The termination fractions are indicated at each step. Not all neutrino types are produced with the same intensity. In Subsection 1.2.1, the neutrino fluxes are broken down by neutrino type and their fluxes are further decomposed into energy spectra.

The study of solar neutrinos with a terrestrial detector implies the knowledge of the source of neutrinos and the understanding of neutrino propagation within the Sun, in the vacuum space between the Sun and the Earth, and then finally through the Earth's atmosphere and internal layers before they reach the detector. The source of neutrinos and the composition of the Sun is introduced in Subsection 1.2.1. The propagation of neutrinos from the source to the detector and neutrino oscillation are briefly explained in Subsection 1.2.2.

\subsubsection{Solar Standard Model}

The standard solar model (SSM) [4-6] is not to be confused with the SM of particle physics. The SSM is a complex model which depends on many input parameters, such as the composition of heavy element abundances in the Sun atmosphere and interior opacities, independently obtained from the observation of the solar surface. The SSM, based on its inputs, evolves the Sun from its formation to the current epoch. Depending on the inputs, a SSM prediction is given a different name. The version used in this thesis is called BS05(OP) [5], after the calculation of Bahcall (B) and Serenelli (S) in 2005 (05), with the opacities provided by the Opacity Project (OP). The BS05(OP) model predicted observables such as the helium abundances at the surface and the depth of the convective zone that agree well with helioseismological measurements. Unfortunately there are many internal parameters in the SSM that 


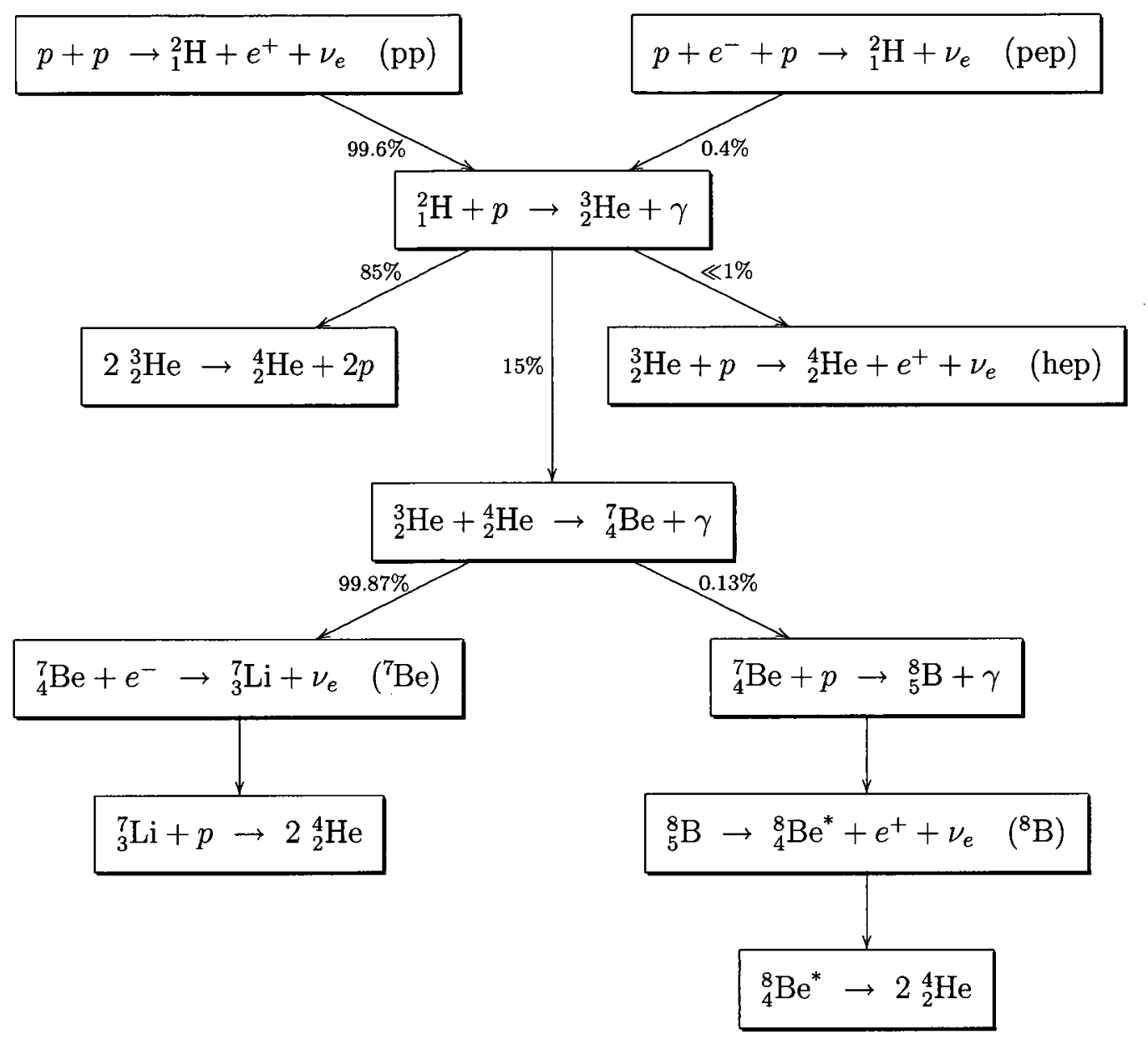

Figure 1.1: Nuclear reactions involved in the proton-proton ( $\mathrm{pp}$ ) fusion chain. The five pp-chain neutrino types, in decreasing order of intensity, are pp, ${ }^{7} \mathrm{Be}, \mathrm{pep},{ }^{8} \mathrm{~B}$, and hep.

cannot be compared to independent measurements.

The parameters of interest which the SSM can predict with good accuracy are the rates of the nuclear fusion reactions that power the Sun. Table 1.1 summarizes the reactions of the solar pp fusion chain and carbon-nitrogen-oxygen (CNO) cycle and lists the predicted neutrino fluxes at the Earth given by the BS05(OP) calculation. The bottom part of Table 1.1 gives the remaining sources of neutrinos, coming from the CNO cycle, that have not been observed to date. Since the elements are heavier in 


\begin{tabular}{|c|c|c|c|c|c|}
\hline \multicolumn{3}{|c|}{ Reaction } & \multirow{2}{*}{$\begin{array}{c}\begin{array}{c}\text { Termination } \\
(\%)\end{array} \\
99.6\end{array}$} & \multirow{2}{*}{$\begin{array}{c}\begin{array}{c}\nu_{e} \text { Flux } \\
\left(10^{10} \mathrm{~cm}^{-2} \mathrm{~s}^{-1}\right)\end{array} \\
.99(1.00 \pm 0.01)\end{array}$} & \multirow{2}{*}{$\begin{array}{c}\text { Type } \\
\text { pp } \\
\text { pep }\end{array}$} \\
\hline $\begin{array}{r}p+p \\
p+e^{-}+p\end{array}$ & $\begin{array}{l}\longrightarrow \\
\text { or }\end{array}$ & $\begin{array}{l}{ }_{1}^{2} \mathrm{H}+e^{+}+\nu_{e} \\
{ }_{1}^{2} \mathrm{H}+\nu_{e}\end{array}$ & & & \\
\hline${ }_{1}^{2} \mathrm{H}+p$ & $\longrightarrow$ & ${ }_{2}^{3} \mathrm{He}+\gamma$ & 100 & & \\
\hline $\begin{array}{l}{ }_{2}^{3} \mathrm{He}+{ }_{2}^{3} \mathrm{He} \\
{ }_{2}^{3} \mathrm{He}+{ }_{2}^{4} \mathrm{He} \\
{ }_{2}^{3} \mathrm{He}+p\end{array}$ & $\begin{array}{l}\longrightarrow \\
\stackrel{\text { or }}{\longrightarrow} \\
\longrightarrow\end{array}$ & $\begin{array}{l}{ }_{2}^{4} \mathrm{He}+2 p \\
{ }_{4}^{7} \mathrm{Be}+\gamma \\
{ }_{2}^{4} \mathrm{He}+e^{+}+\nu_{e}\end{array}$ & $\begin{array}{c}85 \\
15 \\
2 \times 10^{-5}\end{array}$ & $7.93(1.00 \pm 0.16) \times 10^{-7}$ & hep \\
\hline $\begin{array}{r}{ }_{4}^{7} \mathrm{Be}+e^{-} \\
{ }_{3}^{7} \mathrm{Li}+p \\
\\
{ }_{4}^{7} \mathrm{Be}+p \\
{ }_{5}^{8} \mathrm{~B} \\
{ }_{4}^{8} \mathrm{Be}{ }^{*}\end{array}$ & $\begin{array}{l}\longrightarrow \\
\text { or } \\
\longrightarrow \\
\longrightarrow\end{array}$ & $\begin{array}{l}{ }_{3}^{7} \mathrm{Li}+\nu_{e} \\
2{ }_{2}^{4} \mathrm{He} \\
{ }_{5}^{8} \mathrm{~B}+\gamma \\
{ }_{4}^{8} \mathrm{Be}^{*}+e^{+}+\nu_{e} \\
2{ }_{2}^{4} \mathrm{He}\end{array}$ & $\begin{array}{c}15 \\
15 \\
0.0195 \\
0.0195 \\
0.0195\end{array}$ & $\begin{array}{l}4.84(1.00 \pm 0.11) \times 10^{-1} \\
5.69(1.00 \pm 0.16) \times 10^{-4}\end{array}$ & ${ }^{7} \mathrm{Be}$ \\
\hline $\begin{array}{r}{ }_{6}^{12} \mathrm{C}+p \\
13 \mathrm{~N} \\
\end{array}$ & $\longrightarrow$ & $\begin{array}{l}{ }_{7}^{13} \mathrm{~N}+\gamma \\
{ }_{6}^{13} \mathrm{C}+e^{+}+\nu_{e}\end{array}$ & $\begin{array}{l}100 \\
100 \\
\end{array}$ & $3.07\left(1.00_{-0.28}^{+0.31}\right) \times 10^{-2}$ & ${ }^{13} \mathrm{~N}$ \\
\hline $\begin{array}{r}{ }_{6}^{13} \mathrm{C}+p \\
{ }_{7} \mathrm{~N}+p \\
{ }_{8} \mathrm{O}\end{array}$ & $\begin{array}{l}\longrightarrow \\
\longrightarrow\end{array}$ & $\begin{array}{l}{ }_{7}^{14} \mathrm{~N}+\gamma \\
{ }_{8}^{15} \mathrm{O}+\gamma \\
{ }_{7}^{15} \mathrm{~N}+e^{+}+\nu_{e}\end{array}$ & $\begin{array}{l}100 \\
100 \\
100\end{array}$ & $2.33\left(1.00_{-0.29}^{+0.33}\right) \times 10^{-2}$ & ${ }^{15} \mathrm{O}$ \\
\hline $\begin{array}{r}{ }_{7}^{15} \mathrm{~N}+p \\
\\
{ }_{7}^{15} \mathrm{~N}+p \\
{ }_{8}^{16} \mathrm{O}+p \\
{ }_{9}^{17} \mathrm{~F} \\
{ }_{8}^{17} \mathrm{O}+p\end{array}$ & $\begin{array}{l}\overrightarrow{\mathrm{or}} \\
\longrightarrow \\
\longrightarrow\end{array}$ & $\begin{array}{l}{ }_{6}^{12} \mathrm{C}+{ }_{2}^{4} \mathrm{He} \\
\\
{ }_{8}^{16} \mathrm{O}+\gamma \\
{ }_{9}^{17} \mathrm{~F}+\gamma \\
{ }_{8}^{17} \mathrm{O}+e^{+}+\nu_{e} \\
{ }_{7}^{14} \mathrm{~N}+{ }_{2}^{4} \mathrm{He}\end{array}$ & $\begin{array}{l}0.1 \\
0.1 \\
0.1 \\
0.1\end{array}$ & $5.84(1.00 \pm 0.52) \times 10^{-4}$ & ${ }^{17} \mathrm{~F}$ \\
\hline
\end{tabular}

Table 1.1: Reactions producing $\nu_{e}$ 's in the pp chain and CNO cycle. The termination is the percentage of time each reaction occurs for every iteration. The flux values and model uncertainties are taken from the BS05(OP) SSM [5]. The type names are the common abbreviations used to refer to each $\nu_{e}$ reaction. 


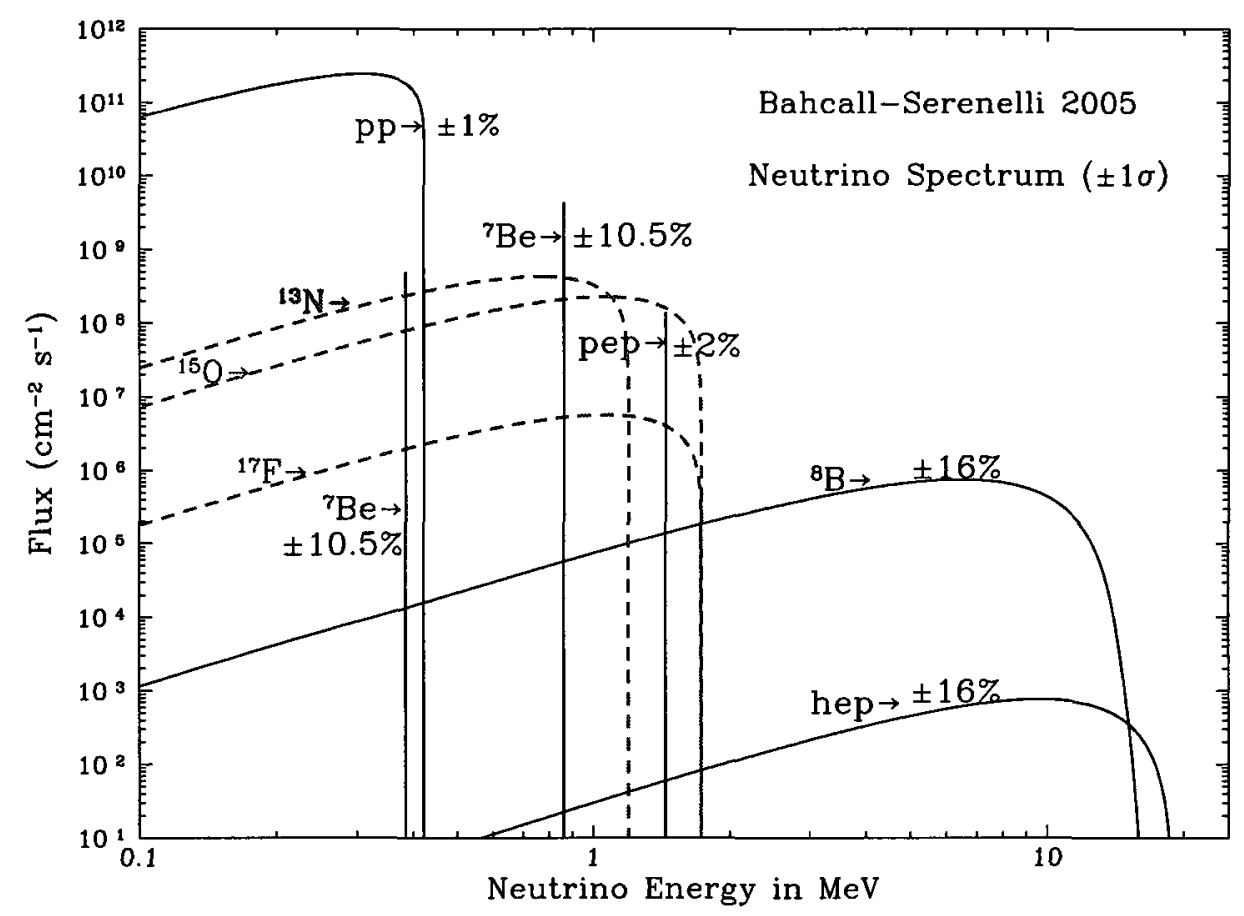

Figure 1.2: Neutrino flux spectra with model uncertainties [5]. Neutrinos produced through the pp chain are shown in continuous lines; those produced by the CNO cycle are shown in dashed lines and have large uncertainties (see Table 1.1). Additional small CNO neutrino fluxes can be produced at higher energy (not shown) [7].

the $\mathrm{CNO}$ cycle, the model prediction of their rates is not as precise as rates resulting from the pp chain. Figure 1.2 shows the spectral distributions for each reaction type. The total neutrino flux is dominated by the pp neutrinos. Unfortunately, most of the current experiments are not sensitive to their low energy $\left(E_{\nu}<0.43 \mathrm{MeV}\right)$, but rather to the less intense high-energy ${ }^{8} \mathrm{~B}$ and hep fluxes.

\subsubsection{Neutrino Oscillation}

The Maki-Nakagawa-Sakata-Pontecorvo (MNSP) matrix is the equivalent of the CKM matrix in the neutrino sector. The parameterization of the MNSP matrix is the same as the CKM matrix, but numerical values of the parameters describing the mixing do 
not have to be the same since there is no such restriction imposed by the SM. However, to incorporate the unknown Majorana-Dirac nature of neutrinos, the general form of the matrix is often written as:

$$
U \equiv U_{\mathrm{MNSP}}=U^{\text {Dirac }} e^{i \lambda_{\mathrm{M}}} \quad U^{\text {Dirac }}=\left(\begin{array}{ccc}
U_{e 1} & U_{e 2} & U_{e 3} \\
U_{\mu 1} & U_{\mu 2} & U_{\mu 3} \\
U_{\tau 1} & U_{\tau 2} & U_{\tau 3}
\end{array}\right)
$$

where the Dirac part has the same form as in Equation (1.1), and where the Majorana part $e^{i \lambda_{M}}$ is a diagonal matrix with two Majorana phases $\lambda_{\mathrm{M}}$. The Majorana phases contribute to an overall phase shift for all neutrino flavors meaning they cannot be measured in oscillation experiments or any experiment involving the kinetic energy of the neutrinos. Therefore in the context of neutrino oscillation, only the Dirac part of the matrix is relevant and the form of the MNSP matrix reduces to $U_{\mathrm{MNSP}}=V_{\mathrm{CKM}}$. A neutrino in a definite flavor state, with flavor $\alpha=e, \mu, \tau$, is a superposition of the mass eigenstates $i=1,2,3$. This also means a state of definite flavor observed in a given interaction does not have a definite mass. The mixing matrix therefore allows one to transform a state from one basis to another:

$$
\left|\nu_{\alpha}\right\rangle=\sum_{i=1}^{3} U_{\alpha i}^{*}\left|\nu_{i}\right\rangle \quad\left|\nu_{i}\right\rangle=\sum_{\alpha} U_{\alpha i}\left|\nu_{\alpha}\right\rangle
$$

and constitutes the basic principle of oscillation calculations, where the mass eigenstates are also the eigenstates of the Hamiltonian of the propagating neutrinos. 


\section{Oscillation in Vacuum}

As a result of solving the Schrödinger equation, the time evolution of a neutrino state is described by a plane wave $\left|\nu_{i}(t)\right\rangle=e^{-i E_{i} t}\left|\nu_{i}\right\rangle$, where $E_{i}=\sqrt{\vec{p}^{2}+m_{i}^{2}}$. The initial condition of the state is assumed to be a definite flavor state, as in the Sun for example, $\left|\nu_{\alpha}(0)\right\rangle=\left|\nu_{e}\right\rangle$. The time evolution of the flavor state is obtained by using Equation (1.3):

$$
\left|\nu_{\alpha}(t)\right\rangle=\sum_{i=1}^{3} U_{\alpha i}^{*} e^{-i E_{i} t}\left|\nu_{i}\right\rangle=\sum_{\beta}\left(\sum_{i=1}^{3} U_{\alpha i}^{*} e^{-i E_{i} t} U_{\beta i}\right)\left|\nu_{\beta}\right\rangle,
$$

and the probability of observing a different flavor is the amplitude squared:

$$
P_{\nu_{\alpha} \rightarrow \nu_{\beta}}(t)=\left|\left\langle\nu_{\beta} \mid \nu_{\alpha}(t)\right\rangle\right|^{2}=\sum_{k, j} U_{\alpha k}^{*} U_{\beta k} U_{\alpha j} U_{\beta j}^{*} e^{-i\left(E_{k}-E_{j}\right) t}
$$

At solar neutrino energies $E_{\nu}$, the neutrinos are ultrarelativistic and the total energy of the mass eigenstates $k, E_{k}$, can be approximated to

$$
E_{k}=\sqrt{E_{\nu}{ }^{2}+m_{k}^{2}}=E_{\nu}\left(1+\frac{m_{k}^{2}}{E_{\nu}{ }^{2}}\right)^{\frac{1}{2}} \simeq E_{\nu}\left(1+\frac{m_{k}^{2}}{2 E_{\nu}{ }^{2}}\right)=E_{\nu}+\frac{m_{k}^{2}}{2 E_{\nu}},
$$

which transforms the difference in the energies of the massive states into a squaredmass difference:

$$
E_{k}-E_{j} \simeq \frac{m_{k}^{2}-m_{j}^{2}}{2 E_{\nu}}=\frac{\Delta m_{k j}^{2}}{2 E_{\nu}},
$$

where the $\Delta m^{2}$ parameters are defined as:

$$
\Delta m_{k j}^{2} \equiv m_{k}^{2}-m_{j}^{2} \quad \text { for } \quad k>j, k \neq j
$$


The ultrarelativistic nature of the neutrinos also allows one to convert times $t$ into distances $L$ which are more convenient for the observation of the oscillation effects given the distance from a detector to the source. The probability of Equation (1.5) then becomes an oscillating function of $L$ and $E_{\nu}$ weighted by the products of the mixing matrix elements:

$$
P_{\nu_{\alpha} \rightarrow \nu_{\beta}}\left(L, E_{\nu}\right)=\sum_{k, j} U_{\alpha k}^{*} U_{\beta k} U_{\alpha j} U_{\beta j}^{*} \exp \left(-i \frac{\Delta m_{k j}^{2} L}{2 E_{\nu}}\right)
$$

The survival probability of flavor $\alpha$ is defined as $P_{\alpha \alpha} \equiv P_{\nu_{\alpha} \rightarrow \nu_{\alpha}}$, as opposed to the transition probability $P_{\alpha \beta} \equiv P_{\nu_{\alpha} \rightarrow \nu_{\beta}}$. Unitarity enforces the conservation of the total probability

$$
\sum_{\beta}^{N_{\nu}} P_{\alpha \beta}=P_{\alpha e}+P_{\alpha \mu}+P_{\alpha \tau}=1
$$

Neutrino oscillations do not allow one to differentiate between Dirac and Majorana neutrinos but can provide evidence of physics beyond the minimal SM through the observation of $P_{\alpha \alpha}$ or $P_{\alpha \beta}$ explained by non-vanishing values of the $\Delta m^{2}$ parameters and mixing matrix elements.

\section{Oscillation in Matter}

Flavor states are affected by effective potentials, due to matter, changing the vacuum evolution of Equation (1.4). The matter potentials arise from coherent interactions with the medium, through forward charged-current (CC) and neutral-current (NC) weak elastic scattering, where coherent means that the medium itself, the matter, is not affected by the passage of the neutrinos. Figure 1.3 shows the interactions of the neutrinos with the components of matter, electrons and nucleons, responsible for the 


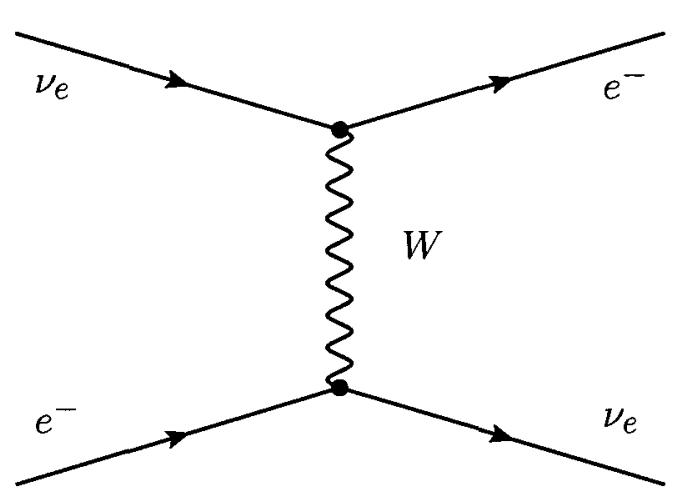

(a) $V_{\mathrm{CC}}$.

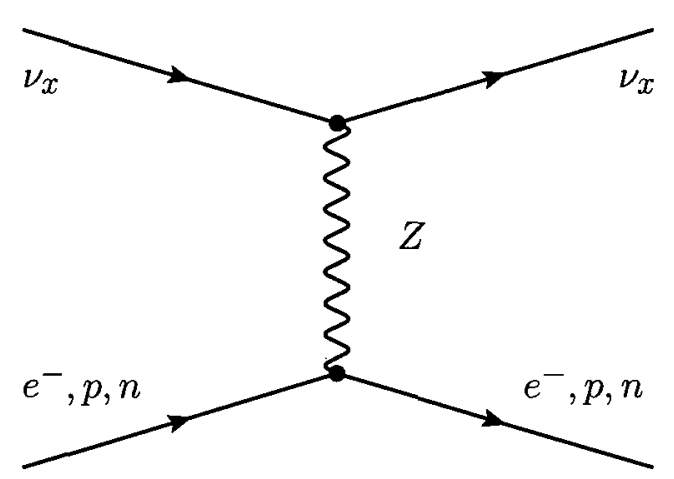

(b) $V_{\mathrm{NC}}$.

Figure 1.3: Feynman diagrams that generate the (a) $\mathrm{CC}$ and (b) NC potentials in matterenhanced oscillation. Here $x=e, \mu, \tau$.

creation of the matter potentials $V_{\mathrm{CC}}$ and $V_{\mathrm{NC}}$.

The coherent scattering effect can enhance the flavor conversion depending on the values of the mixing parameters, which is called the Mikheyev-Smirnov-Wolfenstein (MSW) effect [8-11]. The electron neutrinos produced in the Sun are sensitive to both the $\mathrm{CC}$ and $\mathrm{NC}$ potentials. However, the electrical neutrality of matter in astrophysical environments, such as the Sun, implies that the number of electrons and protons are the same, leaving only the effect of neutrons in the $V_{\mathrm{NC}}$ potential. Furthermore, in the SM, the three flavors of neutrinos are equally sensitive to $V_{\mathrm{NC}}$, which contribute to an overall phase, common to all flavors, which can be removed. Hence the effect of matter in oscillation is taken into account by simply adding the contribution of $V_{\mathrm{CC}}$ to the Hamiltonian, affecting only $\nu_{e}$ 's through the density of electrons in matter. In models that are beyond the SM, such as models with sterile neutrino components [12], the NC potential needs to be re-inserted if the neutrino states are affected differently by $V_{\mathrm{NC}}$. 
The Hamiltonian of the flavor basis $H_{f}$ then takes the form:

$$
H_{f}=\frac{1}{2 E_{\nu}}\left(U M U^{\dagger}+A\right) \quad M=\left(\begin{array}{ccc}
0 & 0 & 0 \\
0 & \Delta m_{21}^{2} & 0 \\
0 & 0 & \Delta m_{31}^{2}
\end{array}\right) \quad A=\left(\begin{array}{ccc}
A_{\mathrm{CC}} & 0 & 0 \\
0 & 0 & 0 \\
0 & 0 & 0
\end{array}\right)
$$

with $A_{\mathrm{CC}}=2 E_{\nu} V_{\mathrm{CC}}=2 \sqrt{2} E_{\nu} G_{\mathrm{F}} n_{e}$, where $G_{\mathrm{F}}$ is the Fermi constant, and $n_{e}$ is the electron density of the medium. Since $G_{\mathrm{F}}$ is very small, the matter potential is also very small, unless the density of electrons becomes very large.

For a given and constant value of $n_{e}$, matter oscillation can be described in the same form as vacuum oscillation, with the effective mixing matrix $U_{m}$ and Hamiltonian $H_{m}=U_{m}^{T} H_{f} U_{m}$ leading to effective mixing angles $\Theta_{j k}$ and mass differences $\Delta M_{k j}^{2}$. The MSW enhancement arises, for solar neutrinos, when the resonance conditions are met:

$$
\tan 2 \Theta_{12}=\frac{\tan 2 \theta_{12}}{1-\frac{A_{C \mathrm{C} C} \cos ^{2} \theta_{13}}{\Delta m_{21}^{2} \cos 2 \theta_{12}}} \quad A_{\mathrm{CC}}^{r} \cos ^{2} \theta_{13}=\Delta m_{21}^{2} \cos 2 \theta_{12}
$$

where $A_{\mathrm{CC}}^{r}$ is the matter potential at resonance. Therefore oscillations in matter are different than in the vacuum and allow the sign of $\Delta m_{21}^{2}$ to be determined based on the values of the mixing angles.

Solar neutrinos are born as $\nu_{e}$ near the core of the Sun, at distances less than $0.3 R_{\odot}$ from its center, and go through regions of large and varying matter densities. The analytic determination of the oscillation probabilities are therefore complicated and need to be calculated by using successive regions of approximate constant density with the Hamiltonian $H_{m}$, or solved numerically using Equation (1.11). 


\subsubsection{Dual Search with Solar Neutrinos}

From the perspective of an observer on Earth, the two fundamental quantities that solar neutrino physicists are interested in, namely the neutrino flux normalization (neutrino brightness) and attenuation (neutrino flavor conversion rate), are correlated. Indeed, the multiplicative effect of increasing or decreasing the normalization and the attenuation simultaneously gives the same effective flux on Earth. The study of solar neutrinos can answer these two correlated questions of Subsections 1.2.1 and 1.2.2 in many, complementary, ways.

The first solution is to build a detector that is not sensitive to neutrino oscillation, that could measure the sum of all active neutrino components. The data from that detector would constrain or confirm the flux normalization, with the limitations associated to the measurement techniques. Unfortunately such detector would not be able to quantify the possible attenuation introduced by neutrino oscillation.

The second solution then comes naturally by asking the help of another experiment, that would provide the missing information on the oscillated flux only. A detector that is sensitive only to $\nu_{e}$ interactions can provide that information. The combined analysis of both experiment's data would simultaneously answer both questions. However, the fact that the two experiments are different requires care in the treatment of the data, and each detector's source of systematic uncertainties must be added, such that the combined analysis would yield an uncertainty that is hard to improve.

The ultimate solution to the above problems in solar neutrino physics comes from the combined analysis of many great experiments, from which one, the Sudbury Neutrino Observatory (SNO) experiment, can measure both the flux normalization and 
attenuation simultaneously. These experiments are briefly introduced in Section 1.3.

\subsection{Solar Neutrino Experiments}

A short description of past, current, and future solar neutrino experiments follows. The listed reactions are the neutrino interaction processes with the target material of a given detector, primarily through the charged-current (CC), neutral-current (NC), and elastic-scattering (ES) channels, involving the exchange of $W$ or $Z$ bosons or both, similar to the diagrams in Figure 1.3. Reported uncertainties are either statistical (stat) or evaluated from known sources of systematic errors (syst).

\subsubsection{First-Generation Experiments}

First-generation experiments consist in counting the by-products of the neutrino interactions after a long exposure of the target. The missing event-by-event information in such experiment only allows for the total neutrino rate to be extracted. Depending on the energy threshold introduced by the cross-section of the reactions, these experiments can be sensitive to all eight solar flux types.

Radiochemical experiments quantify the rate of absorption of $\nu_{e}$ 's on large nuclei, chlorine and gallium, by either counting the rate of decays of the by-products or by detecting Auger electrons. 


\section{Chlorine: Homestake}

The Homestake experiment [13] used the inverse $\beta$-decay reaction:

$$
\mathrm{CC}: \quad \nu_{e}+{ }^{37} \mathrm{Cl} \rightarrow{ }^{37} \mathrm{Ar}+e^{-},
$$

with a threshold energy of $0.814 \mathrm{MeV}$. The threshold results in a limited sensitivity to low-energy neutrinos such as $\nu(\mathrm{pp})$. However the rise of the cross-section with $E_{\nu}$ increases the sensitivity to $\nu\left({ }^{8} \mathrm{~B}\right)$ 's. The final result of the experiment for the $\nu_{e}$ flux, from the analysis of the data taken from March 1970 to February 1994, is $2.56 \pm 0.16$ (stat) \pm 0.16 (syst) $\mathrm{SNU}^{1}$, a factor of 3 lower than the predicted rates without oscillation.

\section{Gallium: GALLEX, GNO, and SAGE}

The three gallium experiments, GALLEX, GNO [14] and SAGE [15], used the reaction:

$$
\mathrm{CC}: \quad \nu_{e}+{ }^{71} \mathrm{Ga} \rightarrow{ }^{71} \mathrm{Ge}+e^{-},
$$

with a threshold energy of $0.233 \mathrm{MeV}$, allowing the rates from all eight solar flux types to be measured. The calculated cross-section predicts that more than $50 \%$ of the total rate comes from $\nu(\mathrm{pp})$ 's therefore probing the pp-chain with great sensitivity.

GNO+GALLEX exposed about 30 tons of Ga from May 1991 to January 1997, leading to a rate of $69.3 \pm 4.1$ (stat) \pm 3.6 (syst) SNU, a factor of 2 lower than the predicted rates without oscillation. The result of SAGE, after taking data from January 1990 to December 2001, is $70.8_{-5.2}^{+5.3}(\text { stat })_{-3.2}^{+3.7}$ (syst) SNU, consistent with

\footnotetext{
${ }^{1}$ The solar neutrino units (SNU) correspond to $10^{-36}$ neutrino captures per atom per second.
} 
GALLEX+GNO. A more recent combined measurement for the $\nu_{e}$ flux of all three Gallium experiments is $66.1 \pm 3.1 \mathrm{SNU}$, where all statistical and systematic uncertainties have been combined [16].

\subsubsection{Second-Generation Experiments}

Experiments of the second generation consist in the real-time observation of Čerenkov

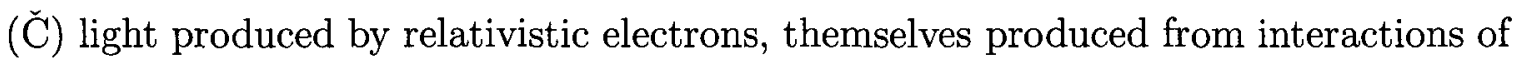
solar neutrinos with a target. Charged particles that have a speed $v>1 / n(c=1)$, where $n$ is the index of refraction of the medium, emit $\check{C}$ radiation in a cone with opening angle $\theta$, with $\cos \theta=1 / n v$. The differential intensity $d N$ of the radiation along the path length $d x$ is

$$
\frac{d N}{d \lambda d x}=\frac{2 \pi \alpha}{\lambda^{2}}\left[1-\left(\frac{1}{n v}\right)^{2}\right]
$$

where $\lambda$ is the wavelength of the light, and $\alpha$ is the fine-structure constant.

In light $\left(\mathrm{H}_{2} \mathrm{O}\right)$ or heavy $\left(\mathrm{D}_{2} \mathrm{O}\right)$ water, the $\check{\mathrm{C}}$ light created in the ultraviolet and visible range, $\lambda \in[300,700] \mathrm{nm}$, is intense enough to be detected by an array of photomultiplier tubes (PMTs). Such system allows one to record each event with information such as the time and PMT charge. The calibration and analysis of the data further lead to the evaluation of the energy of the events and the reconstruction of their position and direction relative to the Sun. Therefore there is much more information available in real-time experiments. However the production of $\breve{\mathrm{C}}$ light limits these experiments to the detection of high-energy neutrinos. 


\section{Light Water: Super-Kamiokande}

The Super-Kamiokande (SK) detector [17] is a large light-water Č detector looking at neutrino events through the reaction:

$$
\mathrm{ES}: \quad \nu_{x}+e^{-} \rightarrow \nu_{x}+e^{-},
$$

where $\nu_{x}$ refers to any active neutrino flavor, with $x=e, \mu, \tau$, but the reaction is predominantly sensitive to $\nu_{e}(\sim 85 \%)$. SK is the successor of the 3-kton Kamiokande experiment, which measured the flux of $\nu\left({ }^{8} \mathrm{~B}\right)$ 's and $\nu$ (hep)'s to be $2.80 \pm 0.19$ (stat) \pm 0.33 (syst) $\times 10^{6} \mathrm{~cm}^{-2} \mathrm{~s}^{-1}[18]$ after 9 years of operation. With its enlarged 50 kton volume, the SK Collaboration has released the results from the two first phases, SK-I and SK-II, with measured fluxes of $2.35 \pm 0.02$ (stat) \pm 0.08 (syst) $\times 10^{6} \mathrm{~cm}^{-2} \mathrm{~s}^{-1}[19]$

and $2.38 \pm 0.05$ (stat) $)_{-0.15}^{+0.16}(\mathrm{syst}) \times 10^{6} \mathrm{~cm}^{-2} \mathrm{~s}^{-1}[20]$, respectively. The predicted rates without oscillation are about twice as large, and the discrepancy is of the order of two standard deviations, in agreement with the conclusion drawn with radiochemical experiments.

\section{Heavy Water: SNO}

The SNO experiment [21] is the subject of this dissertation. SNO is also a secondgeneration experiment, but is more complex than the previous experiments as explained below and in more detail in Chapter 2. The experiment was designed [22] to solve the SNP [23] that past experiments could not resolve completely. SNO employs heavy water made of deuterons $(d)$ as target material, making the experiment 
sensitive to all three active flavors of neutrinos via three reactions:

$$
\begin{array}{ll}
\mathrm{CC}: & \nu_{e}+d \rightarrow e^{-}+p+p-1.442 \mathrm{MeV} \\
\mathrm{ES}: & \nu_{x}+e^{-} \rightarrow \nu_{x}+e^{-} \\
\mathrm{NC}: & \nu_{x}+d \rightarrow \nu_{x}+n+p-2.224 \mathrm{MeV},
\end{array}
$$

where the reaction thresholds are indicated. The ES reaction is similar to SK. The $\mathrm{CC}$ and $\mathrm{NC}$ interactions with the deuteron also lead to the production of $\check{\mathrm{C}}$ light by the electrons in the $\mathrm{CC}$ reaction, and by the neutron capture by-products in the $\mathrm{NC}$ reaction. The SNO experiment is further divided into three phases, each with a different neutron capture process, which are explained in more details in Chapter 2.

The SNO Collaboration has already confirmed the hypothesis of neutrino oscillation with the simultaneous measurement of the $\mathrm{CC}$ and $\mathrm{NC}$ fluxes, in the form of the $\mathrm{CC} / \mathrm{NC}$ ratio $[24-26]$ :

$$
\frac{\Phi\left(\nu_{e}\right)}{\Phi\left(\nu_{x}\right)} \sim \frac{\Phi(\mathrm{CC})}{\Phi(\mathrm{NC})}=0.340 \pm 0.023 \text { (stat) }{ }_{-0.031}^{+0.029} \text { (syst) }
$$

which proves the conversion of $\nu_{e}$ 's into $\nu_{\mu, \tau}$ 's between the neutrino production region in the Sun and the various detectors on Earth, while the NC flux agrees with the SSM predicted flux [27]:

$$
\Phi(\mathrm{NC})=5.54{ }_{-0.31}^{+0.33}(\text { stat })_{-0.34}^{+0.36}(\text { syst }) \times 10^{6} \mathrm{~cm}^{-2} \mathrm{~s}^{-1} .
$$

Since the publication of the solution to the SNP in 2001, the new aim of SNO has been the precise determination of the parameters that describe the oscillation effects 
measured on Earth. This quest is also the main topic of this thesis. Complementary searches included the analysis of solar neutrino rates independently for day- and night-time [28-30] in order to search for evidence of matter effects in the Earth. Secondary analyses also investigated the existence of $\nu$ (hep)'s [31], the periodicity of single neutrino events [32, 33], and the muon flux at SNO [34]. The analysis presented in this thesis directly impacts the latest set of SNO publications [27, 34-36], where the treatment of experimental backgrounds and systematic uncertainties has been refined, leading to the measurement of the CC and ES energy spectra with a low kinetic energy analysis threshold of $3.5 \mathrm{MeV}$. This improved analysis is expected to enhance the precision of the $\mathrm{CC} / \mathrm{NC}$ ratio.

\subsubsection{SNO and the Future}

The success of the neutrino branch of the SSM is experimentally due to SNO which measured the solar $\nu_{e}$ and total fluxes at high energy. However the low energy fluxes cannot be verified with great precision with the current solar neutrino experiments. Therefore a new generation of experiments is needed to measure the fluxes at low energy and verify if the flux normalization and mixing parameters apply at those energies.

The future generation of experiments are also real-time experiments and employ liquid scintillators to detect the light yield created from neutrino interactions. The scintillators work with almost any charged particles, thus both neutrinos or antineutrinos $(\bar{\nu})$ can be detected through an extensive list of reactions. While some experiments can use scintillators to lower their energy threshold to study solar neutrinos, others can detect neutrinos from terrestrial sources, such as those produced by 
beams of decaying pions or created by nuclear power plants. The following describes the current and future prospect experiments that employ these techniques.

\section{Borexino}

The Borexino experiment [37] has the ability to measure the $0.862 \mathrm{MeV}^{7} \mathrm{Be}$ solar neutrinos through the ES reaction. The $\nu\left({ }^{7} \mathrm{Be}\right)$ 's are interesting because of their large flux and single energy value (see Figure 1.2). The Borexino Collaboration measured a $\nu\left({ }^{7} \mathrm{Be}\right.$ )-induced rate of $49 \pm 3$ (stat) \pm 4 (syst) counts per day per 100 ton $[38,39]$ and plan for further precision measurements and the possible observation of higher energy neutrinos.

\section{KamLAND}

The KamLAND experiment [40-42] measures the $\bar{\nu}_{e}$ rate from nuclear reactors in Japan through the reaction $\bar{\nu}_{e}+p \rightarrow n+e^{+}$with a threshold of $1.8 \mathrm{MeV}$. Although the KamLAND detector is not looking for solar neutrinos, the distance that separates the detector from the various sources of $\bar{\nu}_{e}$ is much shorter than the distance SunEarth, which makes the experiment more sensitive to $\Delta m_{21}^{2}$. The current best result from the KamLAND Collaboration is $\tan ^{2} \theta_{12}=0.56_{-0.07}^{+0.10}$ (stat) $)_{-0.06}^{+0.10}$ (syst) and $\Delta m_{21}^{2}=$ $7.58_{-0.13}^{+0.14}$ (stat) $)_{-0.15}^{+0.15}($ syst $) \times 10^{-5} \mathrm{eV}^{2}[42]$.

KamLAND was designed to confirm of the oscillation parameters measured by the solar neutrino experiments. Thus it is standard procedure to combine the measurement of KamLAND with those of solar neutrino experiments to obtain the global fit oscillation parameters, assuming the $\mathrm{CPT}$ invariance which allows one to treat $\nu$ 's and $\bar{\nu}$ 's in the same way. 


\section{Global Fit of Oscillation Parameters}

The excellent agreement between the oscillation parameters obtained with solar $\nu_{e}$ 's and terrestrial $\bar{\nu}_{e}$ 's reflects the great success of the neutrino oscillation experiments. Figure 1.4 shows the combined analysis or global fit of $\tan ^{2} \theta_{12}$ and $\Delta m_{21}^{2}$, in the context of an effective $2 \nu$ oscillation model with an electron and an active component $\left(\nu_{e}, \nu_{a}\right)$, where $\nu_{a}=\nu_{\mu, \tau}$. In this effective model, the effects of $\theta_{13}$ and $\Delta m_{31}^{2}$ are neglected. In addition, neither experiment is sensitive to the transitions generated by $\theta_{23}$ or the phase $\delta$. The best-fit point is $\Delta m_{21}^{2}=7.59 \times 10^{-5} \mathrm{eV}^{2}, \tan ^{2} \theta_{12}=0.468$, and the scale of the $\nu\left({ }^{8} \mathrm{~B}\right)$ flux is $f_{8_{\mathrm{B}}}=0.864$ with respect to the BS05(OP) model prediction.

Figure $1.5 \mathrm{a}$ is a summary of the rates measured by solar neutrino experiments relative to the $\mathrm{BS} 05(\mathrm{OP})$ model prediction as a function of operation time. The model prediction varies from one experiment to another due to their different sensitivities to various fluxes and energy ranges. The discrepancy of all rates, except for SNO's $\mathrm{NC}$, is due to oscillations. Figure $1.5 \mathrm{~b}$ shows the same rates after correcting for the effect of $\bar{P}_{e e}$, the average survival probability for each experiment. The mixing parameters used to generate the probabilities were those of KamLAND such that the corrected rates were independent of any solar neutrino data. The corrected rates have come closer to the SSM prediction, but differences still exist, especially the $\mathrm{CC}$ rates of SNO. As for the confidence regions in Figure 1.4, this might suggest a residual discrepancy between the solar experiments and KamLAND. This motivates a more precise analysis of the oscillation parameters, and a study of the differences between the $\nu_{e}$ in solar experiments and $\bar{\nu}_{e}$ in terrestrial experiments. In particular, the analysis presented in this thesis also includes the sub-leading effect of the $\theta_{13}$ 

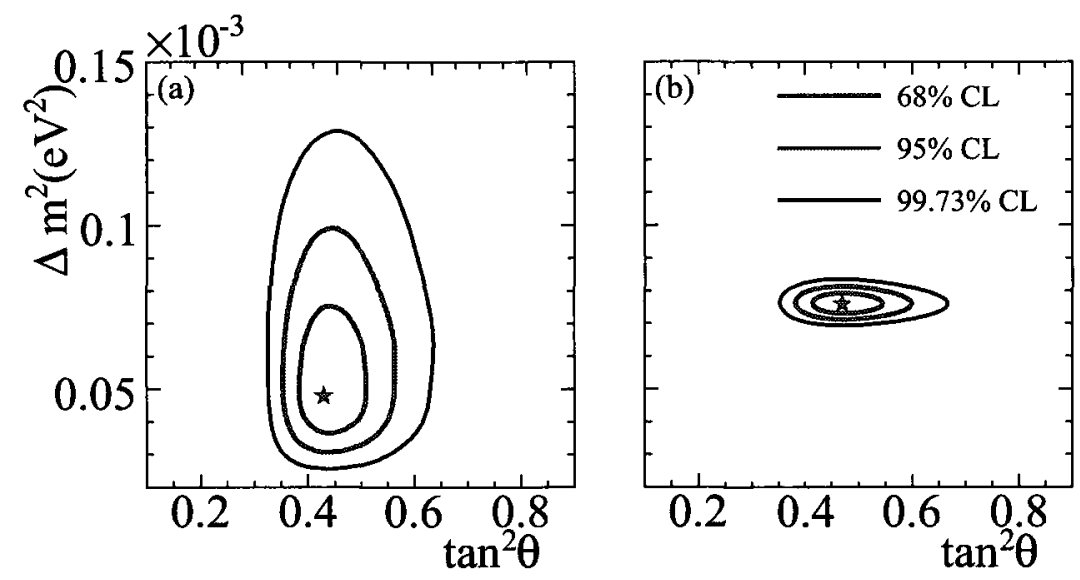

Figure 1.4: Confidence levels (CL) of the $2 \nu$ oscillation parameters, where $\tan ^{2} \theta \equiv \tan ^{2} \theta_{12}$ and $\Delta m^{2} \equiv \Delta m_{21}^{2}$, obtained with (a) the solar neutrino experiments and (b) solar with KamLAND. Figure from [27].

and $\Delta m_{31}^{2}$ in a full $3 \nu$ oscillation analysis, which can potentially improve the overall agreement when performing global fits.

\section{Current and Future Experiments}

After the success of SNO, SK, KamLAND, and radiochemical experiments at determining the first-order oscillation parameters, $\theta_{12}$ and $\Delta m_{21}^{2}$, several experiments, the third generation, have been proposed to measure either the low energy solar neutrinos or the precise effect of the oscillation to higher orders in $\theta_{13}$ and $\Delta m_{31}^{2}$. Beyond oscillation experiments, others are proposed to investigate the mass hierarchy $\left(m_{3}>m_{2}>m_{1}\right.$ or $\left.m_{2}>m_{1}>m_{3}\right)$, determine the neutrino nature (Majorana or Dirac), or measure the absolute masses of the neutrinos. The following briefly highlights these experiments.

Terrestrial oscillation experiments are directed towards the precise measurement of the remaining oscillation parameters. Similar to KamLAND, experiments looking at $\bar{\nu}_{e}$ fluxes at nuclear reactors can vary their sensitivity to the mixing angles by 


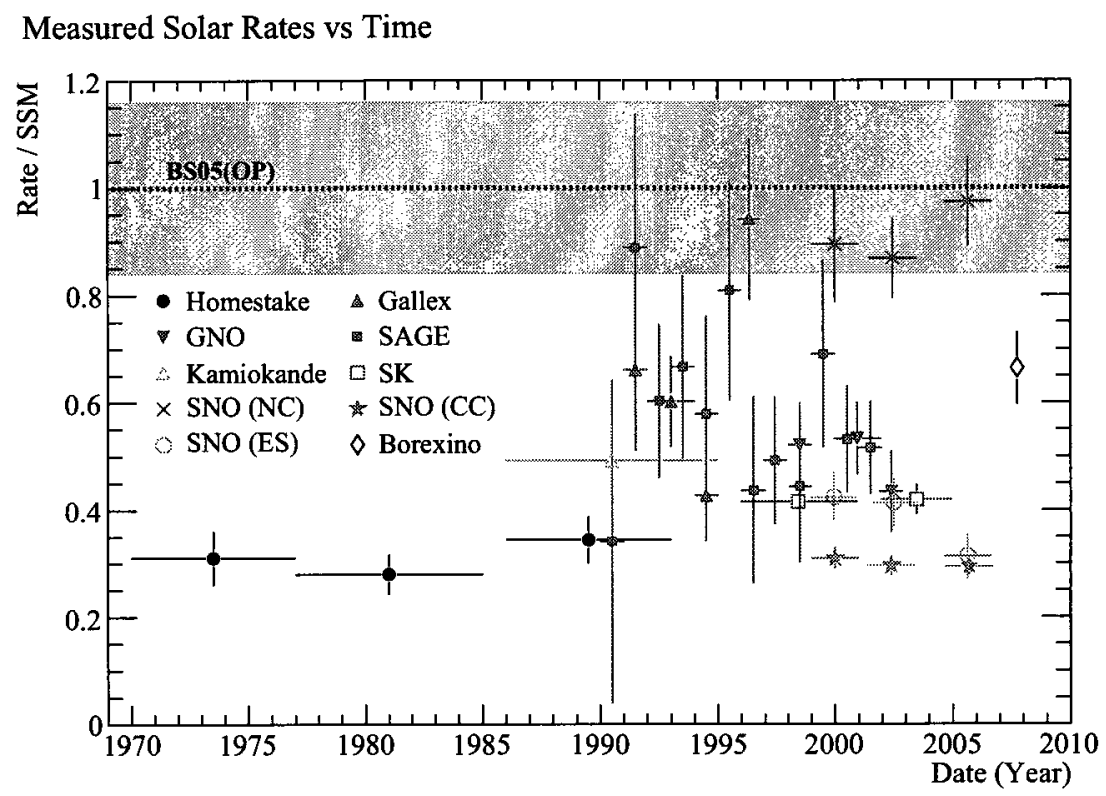

(a) Rate over the SSM prediction in arbitrary units.

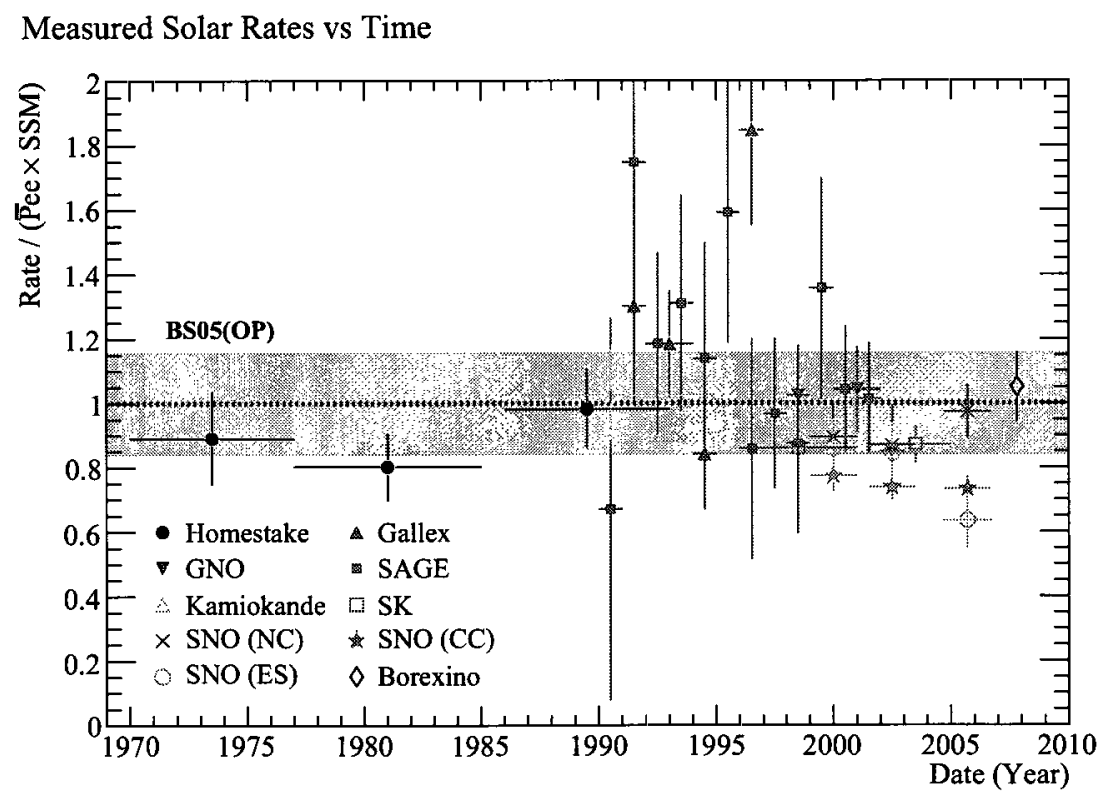

(b) Corrected rate using the KamLAND best-fit parameters.

Figure 1.5: Ratio of experimental rates to the SSM prediction. The error band shown is the $16 \%$ error on the ${ }^{8} \mathrm{~B}$ flux in the BS05(OP) model. (a) The reported rates are plotted without oscillation corrections. (b) The effect of the average survival probability, $\bar{P}_{e e}$, has been removed. The oscillation parameters of KamLAND [42] were assumed. Figures adapted from [43]. 
choosing the optimal distance to the source. Namely, the CHOOZ experiment [44] has determined the best limit on $\theta_{13}$ by measuring the flux at a distance of about $1 \mathrm{~km}$. Their limit is $\sin ^{2} 2 \theta_{13}<0.19$ at the $90 \%$ confidence level. The successor, Double-CHOOZ [45], will reuse the $\mathrm{CHOOZ}$ detector with the addition of a neardetector to measure the flux simultaneously at two different distances, canceling the effects of the systematic uncertainties and improving the sensitivity to small values of $\theta_{13}$.

Longer baseline experiments measure the flux of neutrinos produced in accelerators. In the $\mathrm{K} 2 \mathrm{~K}$ [46] and $\mathrm{T} 2 \mathrm{~K}$ experiments in Japan, protons are accelerated and directed onto a target which results in an intense beam of pions which decay into muons and $\nu_{\mu}$ 's. The neutrinos are focused towards the SK detector such that the effect of oscillation can be measured over distances of about $250 \mathrm{~km}$. MINOS [47] is a similar experiment with a baseline of $730 \mathrm{~km}$ which recently started to analyze data.

$\mathrm{SNO}+[48]$ is a scintillator experiment that plan to re-use the SNO detector. Because there are not enough nuclear plants around Sudbury in Canada to study $\bar{\nu}_{e}$ 's, the experiment is rather oriented towards the measurement of low energy pep and $\mathrm{CNO}$ neutrinos. There are also plans for SNO+ to look for a distinct neutrino-less double-beta decay signal and potentially identify the Majorana nature of neutrinos. The EXO [49] experiment, to name only this one, plans to use enriched Xenon and time-projection chambers to look for the distinctive signal of barium atoms when Xenon decays through the neutrino-less double-beta channel as well. Both SNO+ and EXO will improve the limits on the smallness of the absolute neutrino mass scale if the neutrino is a Majorana particle. 


\subsection{Synopsis}

The solar neutrino experiments were just the start of a rich and wonderful quest for the precise characterization of neutrinos in general and the search for answers to open questions concerning the SM of particle physics.

This thesis is aimed at the measurement of the survival probability and the determination of the oscillation parameters relevant to solar neutrinos detected by the SNO experiment. Especially an attempt is made to set a limit on $\theta_{13}$ with solar neutrino data. This angle is expected to be small and is the last of the MNSP matrix to be measured. A value of $\theta_{13}$ different from zero would open the possibility of leptonic CP-symmetry breaking through the phase $\delta$, which has not been observed yet because of the smallness of $\theta_{13}$. Although terrestrial and future experiments are dedicated to this precise measurement, the solar neutrino data can also constrain its value, as it will be shown in Chapter 7 .

Chapter 2 describes the main features of the SNO detector. Chapter 3 details the calibration work done in preparation for the operation of SNO in its third and final phase. Chapter 4 contains the details of the optical calibration of the SNO detector for the latter phase. The main discussion being neutrino astrophysics, the technical topics in Chapters 3 and 4 may be skipped without loss of generality. Chapter 5 summarizes the statistical separation method employed to find neutrino events in the context of the improved low energy threshold analysis. Finally, Chapters 6 and 7 present the survival probability analyses of the SNO energy spectra, where the survival probability and mixing parameters are extracted, and Chapter 8 summarizes the achievements. 


\section{CHAPTER 2}

\section{The SNO Detector}

The Sudbury Neutrino Observatory (SNO) was located in Vale INCO's Creighton mine near Sudbury, Ontario, Canada. At $2 \mathrm{~km}$ deep underground, the rock overburden of $6010 \pm 20 \mathrm{~m}$ of water equivalent resulted in less than 80 cosmic ray muons passing through the detector everyday. As depicted in the schematic of Figure 2.1a, the SNO detector consists of two almost-concentric spheres. The innermost sphere is a $12 \mathrm{~m}$ diameter and $5.5 \mathrm{~cm}$-thick acrylic vessel (AV) containing SNO's unique target mass of 1000 tonnes of ultra-pure heavy water $\left(\mathrm{D}_{2} \mathrm{O}\right)$. The neck at the top of the AV opens up to the deck clean room where sources were inserted into the detector for calibration. The AV is surrounded by light water $\left(\mathrm{H}_{2} \mathrm{O}\right)$ and then by a $17.8 \mathrm{~m}$ diameter outer geodesic sphere, the PMT support structure (PSUP), which holds 9438 photomultiplier tubes (PMTs) oriented towards the $\mathrm{D}_{2} \mathrm{O}$ target. The inner ultra-pure $\mathrm{H}_{2} \mathrm{O}$ volume of 1700 tonnes between the AV and PSUP shields the $\mathrm{D}_{2} \mathrm{O}$ target volume from radioactivity in the PSUP and PMTs. Past the PSUP, 5300 tonnes of $\mathrm{H}_{2} \mathrm{O}$ fills the remainder of the cavity and also acts as a shield against radioactivity from the cavern walls, particles, and radiation entering the detector from outside.

The SNO detector relies on the PMTs to see the signals due to neutrino interactions in the $\mathrm{D}_{2} \mathrm{O}$. Figure $2.1 \mathrm{~b}$ shows a typical SNO PMT with specifications. The Hamamatsu R1408 tubes, that are made of Schott glass to minimize the intrinsic 
radioactivity, provide a short photo-electron $(\mathrm{PE})$ transit time of $30 \mathrm{~ns}$ with a resolution of $1.5 \mathrm{~ns}$ and a maximum detection efficiency of $21.5 \%$ at a wavelength of $420 \mathrm{~nm}$. With the PMTs alone, the photo-cathode coverage of the detector would be $31 \%$. However, each PMT is mounted on the PSUP with a light reflector of $26.9 \mathrm{~cm}$ in diameter which increases the overall coverage to $54 \%$ and decreases the chances of detecting light created by radioactive decays in the mine rock, beyond the PSUP. In addition to the inward facing tubes, there are 91 outward looking (OWL) PMTs pointing towards the outer light water volume. The OWLs provide the important tag of events that originate from the detector surroundings such as products created by cosmic ray muons.

At a latter stage of the experiment, an array of ${ }^{3} \mathrm{He}$ proportional counters was introduced in the AV to observe NC events independently from the PMTs. The total array length was $398 \mathrm{~m}$, including 36 independent channels to detect neutrons and 4 additional channels to identify backgrounds. The array is described in more details in the following sections. More details on the components of the SNO detector can be found in [21].

The remainder of this chapter discusses the various systems that composed the SNO detector, separately, with a conclusion on the simulation software and its relation with the analysis presented in the next chapters. Section 2.1 defines the various configurations of the SNO detector and lists their main characteristics. Sections 2.2 and 2.3 explain the PMT and NCD systems of the SNO detector. Then Section 2.4 lists the backgrounds and the roles of the water systems that were in place to control them, and Section 2.5 is a description of the simulation software. 


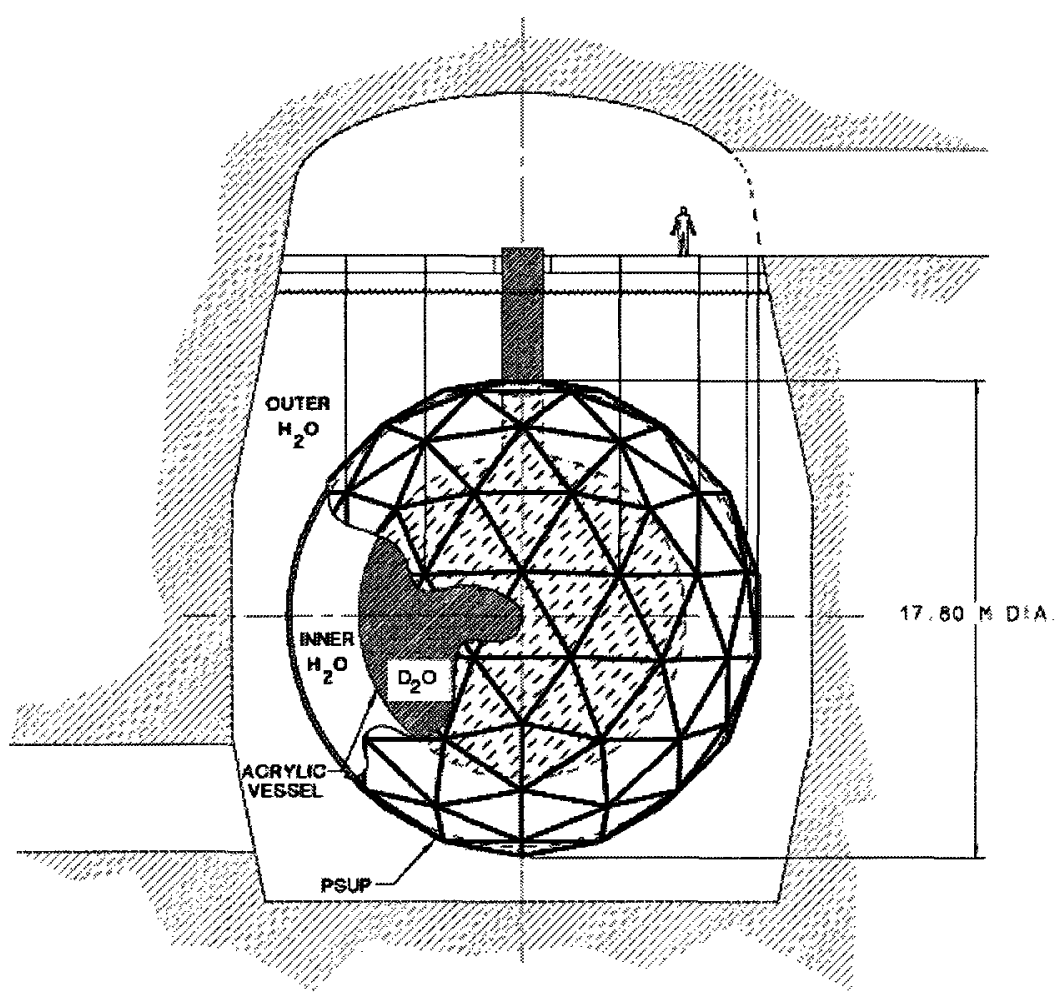

(a) Schematic view of the SNO detector.

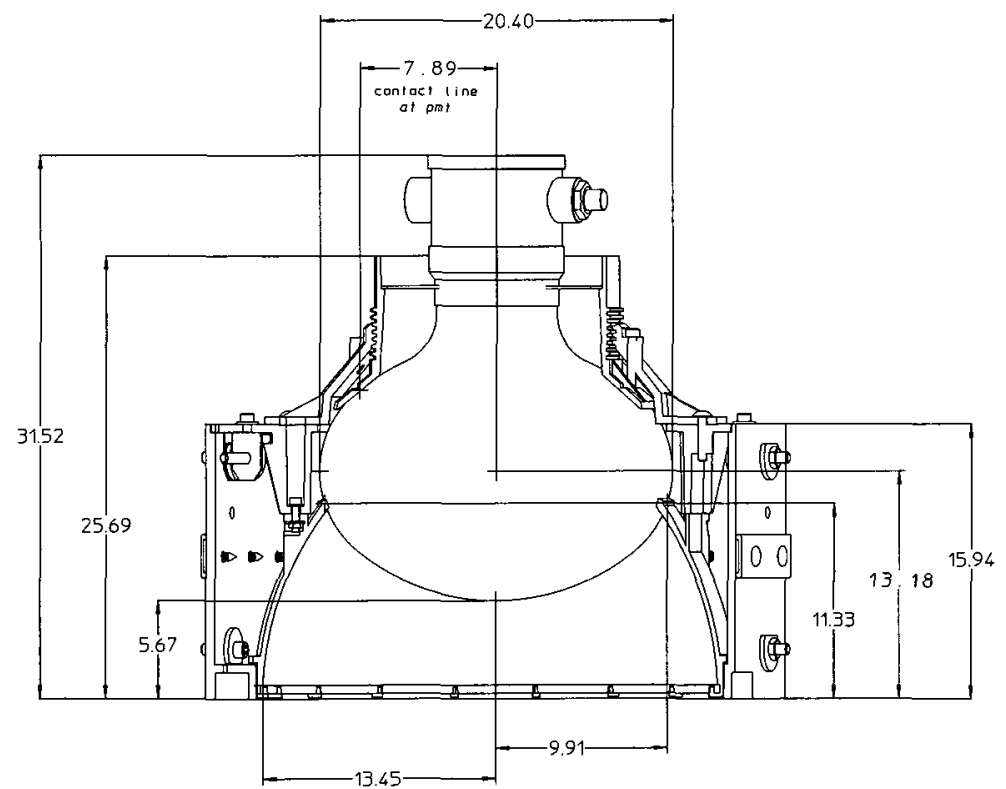

(b) A SNO PMT and its reflector assembly. Dimensions are given in $\mathrm{cm}$.

Figure 2.1: The SNO detector and a SNO PMT with its reflector assembly [21]. 


\begin{tabular}{|l|c|c|c|}
\hline \hline Phase & $\mathrm{D}_{2} \mathrm{O}$ & Salt & NCD \\
\hline Neutron Capture & $n+\mathrm{D}_{2} \mathrm{O}$ & $n+{ }^{35} \mathrm{Cl}$ & $n+{ }^{3} \mathrm{He}$ \\
Cross Section (b) & $5 \times 10^{-4}$ & 44 & 5333 \\
Signature & $6.25 \mathrm{MeV} \gamma$ & $8.6 \mathrm{MeV} \gamma$ 's & $573 \mathrm{keV} \mathrm{p}+191 \mathrm{keV}{ }^{3} \mathrm{H}$ \\
Dates of Operation & $11 / 1999$ to $05 / 2001$ & $07 / 2001$ to $08 / 2003$ & $11 / 2004$ to $11 / 2006$ \\
Livetime (days) & 279.27 & 389.85 & 385.17 \\
\hline \hline
\end{tabular}

Table 2.1: The phases of the SNO experiment. 1 barn $(b)=10^{-24} \mathrm{~cm}^{2}$.

\subsection{Detector Configurations}

The SNO experiment consists of three sub-experiments sharing the common goal of measuring the NC flux with different neutron detection techniques, while still allowing the observation of CC and ES events. The sub-experiments, also called phases, span many years of data taking, with a total livetime of $1054.29 \pm 0.03$ days. The names, dates of data acquisition, and main features of the various phases are given in Table 2.1 .

\subsubsection{Pure Heavy Water Phase}

The pure heavy water phase, also called $\mathrm{D}_{2} \mathrm{O}$ phase, is defined as the period of running the SNO detector between November 1999 and May 2001 with $\mathrm{D}_{2} \mathrm{O}$ molecules as a target for incoming solar neutrinos. The NC reaction cross-section of $5 \times 10^{-4} \mathrm{~b}$ includes the pure cross-section on deuterons, $\nu_{x}+d$, and the efficiency of the $\mathrm{D}_{2} \mathrm{O}$ molecules to capture neutrons and release a mono-energetic $\gamma$-ray of $6.25 \mathrm{MeV}$. The Compton-scattering of the $\gamma$-ray with one of the surrounding electrons then initiates the production of $\check{C}$ light that is detected by the PMTs. 
From an analysis point of view, the NC, CC, and ES events are practically impossible to distinguish and a statistical separation technique is needed to extract the relative fractions of each class of events. This technique is explained in Chapter 5. The complete archival of the first phase of SNO can be found in [26].

\subsubsection{Salt Phase}

The salt phase is defined as the period of running the SNO detector between July 2001 and August 2003 with 2 tonnes of clean $\mathrm{NaCl}$ dissolved within the $\mathrm{D}_{2} \mathrm{O}$ volume. While the $\nu_{x}+d$ cross-section is the same as in the $\mathrm{D}_{2} \mathrm{O}$ phase, the $\mathrm{Cl}$ increases the capture rate of the released neutrons, and facilitates their observation with a high total $\gamma$ energy of $8.6 \mathrm{MeV}$. The ${ }^{35} \mathrm{Cl}$ in the $\mathrm{NaCl}$ captures the free neutron and excites to ${ }^{36} \mathrm{Cl}^{*}$ before the emission of multiple $\gamma$-rays. The latter create events that are more isotropic in the detector making the identification of the $\mathrm{NC}$ events somewhat easier compared to the $\mathrm{D}_{2} \mathrm{O}$ phase. More information about the technical details, analysis methods, and physics results of the salt phase can be found in [30].

\subsubsection{Neutral Current Detector Phase}

The Neutral Current Detector (NCD) phase, is defined as the period of running the SNO detector between December 2004 and December 2006 after 40 NCDs were installed inside the $A V$. The neutron detectors were submerged in the $\mathrm{D}_{2} \mathrm{O}$, after the desalination period. The installation required the disassembly of the calibration systems on top of the detector, such that the NCDs could be introduced through the AV chimney. This disruption period is referred to as the NCD commissioning phase, which started in October 2003 and ended in August 2004. The NCDs are 
lighter than the heavy water thus they were attached at the bottom of the AV with strings holding them to specific AV panels that have anchors. Figure 2.2 shows the NCD array configuration in the detector's $x y$-plane, to scale, with the AV and PSUP spheres.

The NCDs are cylindrical proportional counters filled with ${ }^{3} \mathrm{He}$ gas, as depicted in Figure 2.3. NC events arise from the neutron interaction with ${ }^{3} \mathrm{He}$ :

$$
n+{ }^{3} \mathrm{He} \rightarrow p+{ }^{3} \mathrm{H}+764 \mathrm{keV},
$$

where the produced proton $(573 \mathrm{keV})$ and triton $(191 \mathrm{keV})$ ions have enough kinetic energy to create over $20 \mathrm{k}$ electron-ion pairs. The drift and multiplication of the electrons at the anode wire results in a distinct and measurable signal. More details on the NCD installation procedure and operation can be found in $[50,51]$.

\subsection{PMT System}

The PMT system was in operation in all three phases of the SNO experiment. Subsection 2.2.1 introduces the PMT electronics and trigger systems. Extensive details of the SNO PMT electronics and trigger can be found in [52]. Then Subsection 2.2.2 is an overview of the calibration methods and hardware used to deploy calibration sources in the detector.

\subsubsection{Electronic and Trigger Systems}

Cerenkov light is detected by 9438 PMTs in SNO. The resulting pulses travel from the base of the PMTs to the front-end electronics (FEE) via $32 \mathrm{~m}$ of waterproof coaxial 


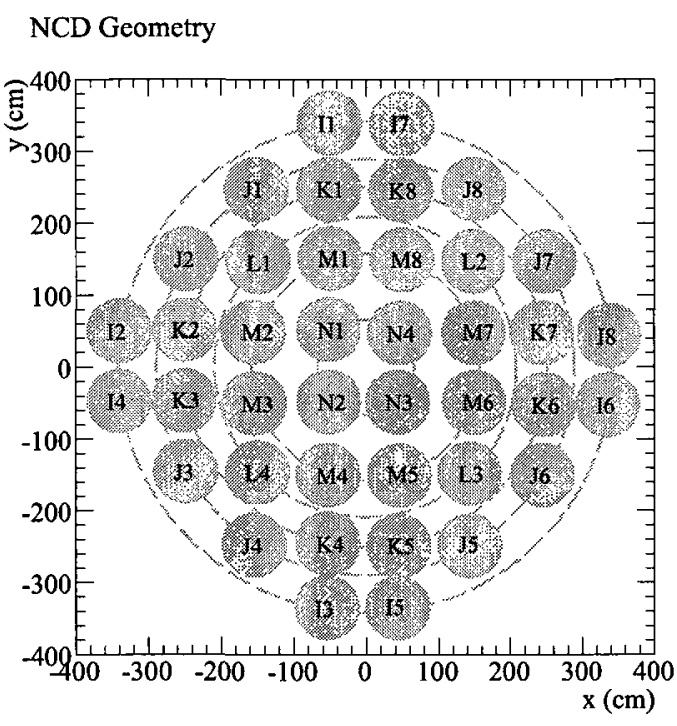

(a) NCD Array.

\section{SNO Geometry}

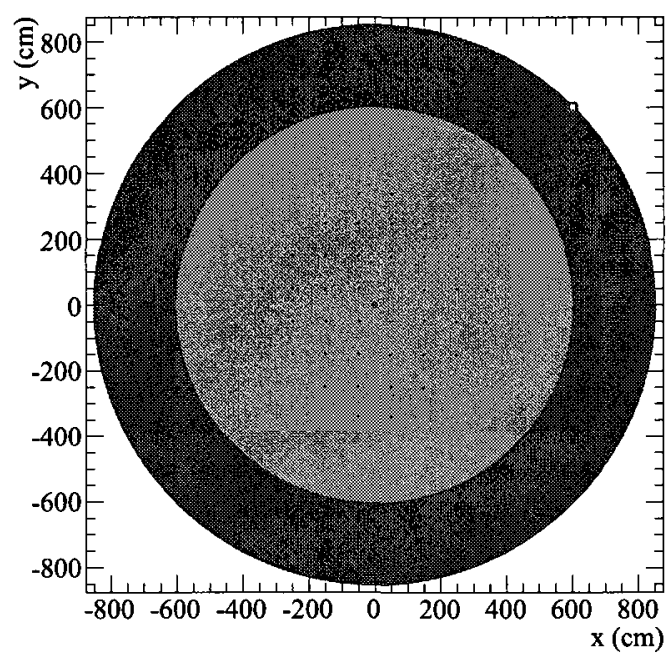

(b) NCD Array in SNO.

Figure 2.2: The SNO geometry during the NCD phase, viewed from the top of the detector, including the 40 parallel NCD counters deployed in the AV volume. (a) The name of each counter is shown, with their identifying rings. The array is not perfectly symmetric. (b) Also shown, to scale, the laserball source (center point), the AV at $r=600 \mathrm{~cm}$, the PSUP at $r=850 \mathrm{~cm}$, and a PMT on the PSUP at $x=y \simeq 600 \mathrm{~cm}$.

cable. The main purpose of the electronics is to determine if a PMT signal is above threshold, and if so, to produce multiple trigger signals to be summed over all electronic channels. When a pre-determined number of PMTs have fired in coincidence, a global trigger (GT) is initiated and the FEE digitizes the integrated charge and time of all PMTs. The digitized information is then read out by the Data Acquisition System (DAQ) to be stored.

The FEE consists of 19 crates each with 512 channels in 16 boards of 32 channels. The PMT cables are assembled onto a paddle card in groups of eight. Four paddle cards, or 32 channels, are connected to the PMT Interface Card (PMTIC), which provides high voltage (HV) to the PMTs. There are 16 PMTICs in a single crate. Once the HV has been subtracted from the PMT signal, the signal is delivered to 


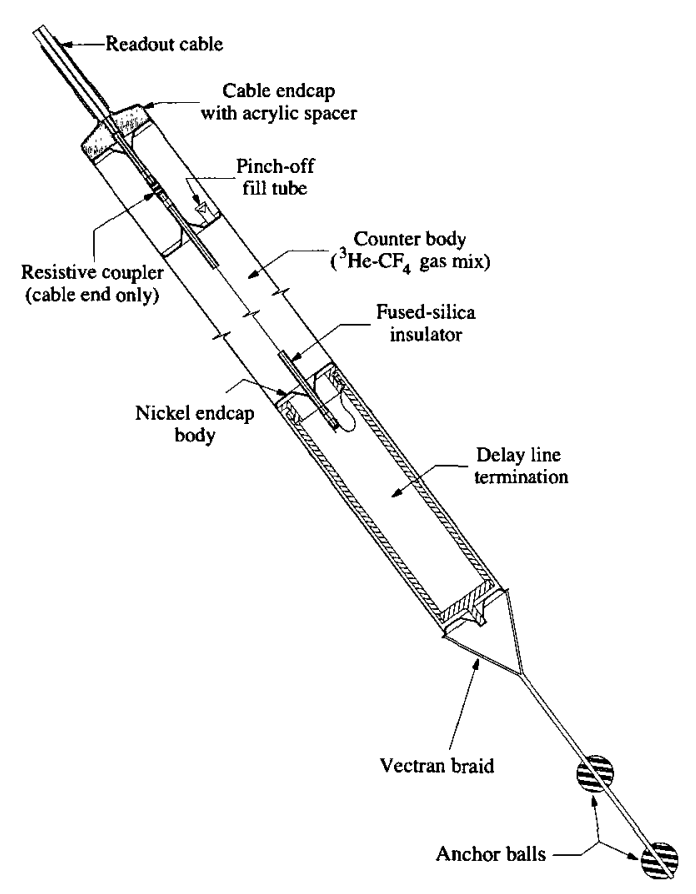

Figure 2.3: Schematic of a NCD string with readout cable, active region, delay line, and anchor system. The outer cylinder radius is $R_{\mathrm{ncd}}=2.579 \mathrm{~cm}$. The counter body, not to scale on the figure, consisted of 9 to $11 \mathrm{~m}$ long active regions, made of three or four inter-connected individual gas volumes.

one of the 16 motherboards, which are directly interfaced with the PMTICs. There are four daughterboards connected to each motherboard. On each daughterboard, custom application-specific integrated circuits (ASICs) determine if the PMT signal is above threshold.

When the signal passes the ASIC requirements, four main trigger signals are produced: long and short time coincidence, and high and low gain charge trigger pulses. The four trigger types are continuously summed by the Crate Trigger Card (CTC) for each crate. The summed trigger signals from all crates are sent to an analog master trigger card (MTC/A) to determine the sum of all 19 crates. When the latter is above the limit set on the MTC/A by the DAQ, the information is sent to the digital 
master trigger card (MTC/D). The MTC/D determines if the trigger that crossed threshold is enabled by the DAQ and if so, initiates the GT which is sent back to all crates via the CTC. Once the FEE receives the GT, the integrated charge and time for the hit PMTs, calculated by the ASICs on the daughterboard, are digitized and stored temporarily on the motherboard. The PMT information is read from the motherboard's memory by the DAQ via a pair of translator cards (XL1 and XL2).

The MTC/D sends GTs to all crates in synchronization with a $50-\mathrm{MHz}$ clock to allow the time of the trigger to be known. The time is also recorded from a commercial global positioning system (GPS) 10-MHz clock. The MTC/D records the state of all raw triggers at the time of the GT. After sending a GT, the MTC/D rejects any other GT within a gate of $440 \mathrm{~ns}$, allowing for scattered and reflected light to be included in the event information.

\section{Trigger Types}

The goal of any trigger system is to select events that are of physics interest and notify the DAQ to store those events. The SNO trigger system was an analog system designed with several possible trigger types: number of hit PMTs within a given time window, integrated charge in the PMTs, software, and external triggers. Each trigger is listed below with brief explanations.

The N100 trigger is the main physics trigger type and is based on the number of hit PMTs. When the discriminator fires, each channel produces a square pulse $93 \mathrm{~ns}$ in width. All channels are summed to determine if the preset threshold is crossed. Throughout the experiment, 16 hit PMTs within a 100 ns coincidence time window were required before issuing a GT. The $100 \%$ trigger efficiency for N100 was reached 
at 23 hits or an energy of about $3 \mathrm{MeV}$ [26].

The N20 trigger is similar to N100 except that the pulse width is $20 \mathrm{~ns}$. The N20 was designed to be a low energy background trigger but due to electronic pick-up, the hit threshold for the $\mathrm{D}_{2} \mathrm{O}$ and salt phases was the same as the $\mathrm{N} 100$ trigger.

The OwlN trigger is identical to the N100 trigger, except that it is specific to the OWL tubes. The OWL triggers are separate from the inward channels and are efficient at tagging particles entering the detector from outside, such as muons.

The charged-based triggers, ESumHi, ESumLo, OwlEHi, and OwlELo, are the low- and high-charge triggers for inward and OWL PMTs, respectively. For each PMT, an analog copy of each pulse is produced. The pulses from all tubes are summed together to provide a trigger based on the amount of charge deposited in the detector. Since the shape of the pulse is not sharp in time like the N100 trigger, the ESum triggers are too slow for online physics event tagging, but remain useful for offline detector diagnostics and identification of instrumental and non-physics backgrounds.

Other triggers include fixed interval, software, and external triggers. The Pulsed Global Trigger (PGT) is a pulser-generated calibration trigger. This $5-\mathrm{Hz}$ trigger was used to measure the PMT noise hits in the detector and served as a verification for livetime measurements. Software initiated triggers only marked the beginning and end of a run. In addition, the trigger system can handle up to eight external triggers. These were used to trigger on events from tagged calibration sources. Among these, the asynchronous external trigger could send GTs independently of the 50-MHz clock and was mainly designed to trigger on laserball events used to calibrate the detector response. 


\subsubsection{Calibrations}

Calibrations of the SNO detector are essential in understanding both signal and background events. They consist of electronic calibrations, through which the basic charge and timing information of the PMTs are obtained, and source calibrations which, after offline analysis, determine the response of the detector to signal and background events.

\section{Electronic Calibrations}

The calibration of the PMT electronics is performed through electronics and PMT calibrations. Electronic calibration (ECA) [53] consists in two sets of dedicated runs. The pedestal run calculates the pedestal value of the charge analog-to-digital conversion (ADC) and the time-slope run calculates the slope of the time-to-amplitude conversion (TAC). The DAQ sends programmed pulses to a specific channel, causing its discriminator to fire, to determine the pedestal values and TAC slopes. Each pulse is followed at a fixed delay by a GT. The time slope is measured by varying the delay between the input pulse and the GT. Since the pedestal values and TAC slope may change over time, these calibration runs were done on a bi-weekly and bi-monthly basis for the pedestal and TAC measurements, respectively.

PMT calibration (PCA) [54] mimics data taking to ensure proper timing of the PMT system. During the PCA, the PMT discriminator fires when the leading edge of a pulse crosses the voltage threshold. The recorded time thus depends on the rise time of the pulse. To remove PMT-to-PMT timing differences due to possible discriminator walk, an almost-isotropic source, the laserball (LB), was deployed in the center of the detector to measure the relationship between PMT charges and 
discriminator firing time. PCA runs were performed on a monthly basis.

\section{Detector Calibrations}

The SNO Collaboration employs several calibration sources to measure the overall PMT collection efficiency, PMT and concentrator angular response, optical attenuations, energy response as a function of detector position for both electrons and neutrons, and detector acceptance to low energy backgrounds. The SNO calibration system is flexible, allowing to insert multiple different source types with various geometries and energy ranges into the detector, both inside and outside the AV. As seen in Figure 2.4a, the calibration system had the capability of deploying sources along the central $z$-axis, in the $x z$ - and $y z$-planes, and also outside of the AV using guide tubes that are parallel to the $z$-axis.

The main calibration sources are described briefly below. Emphasis is put on the laserball and laser systems as an introduction for the optical calibration techniques presented in Chapters 3 and 4.

\section{Laser System and Laserball}

A $\mathrm{N}_{2}$ laser coupled to dyes provides intense almost-monochromatic light at six wavelengths in the sensitive range of the PMTs. Table 2.2 gives the central wavelengths obtained and their spread due to the dyes. The laser system allows the selection of neutral densities from two wheels to reduce the intensity of the laser light before it is directed to an optical fiber which brings the light to the LB. This step was necessary during optical calibrations, where the total intensity of the light was adjusted at the level of a single photo-electron (PE) per PMT, corresponding to almost 300 hit 


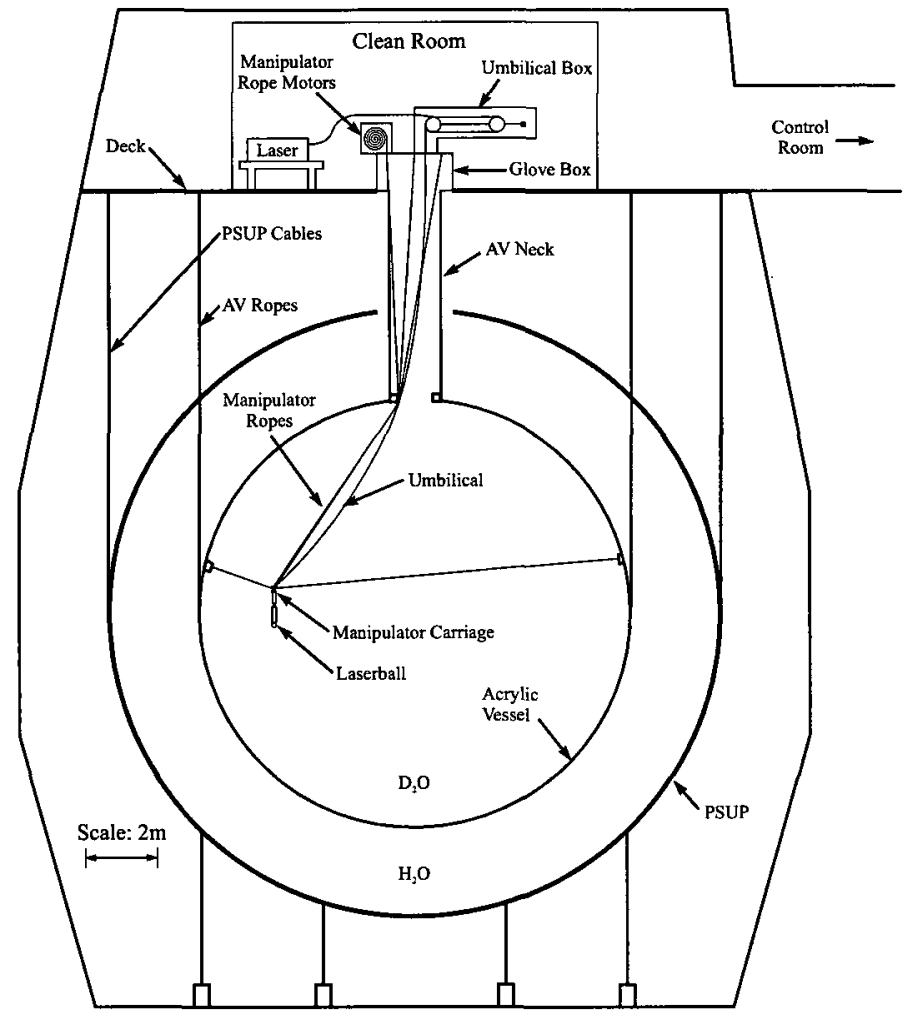

(a) Source manipulator system.

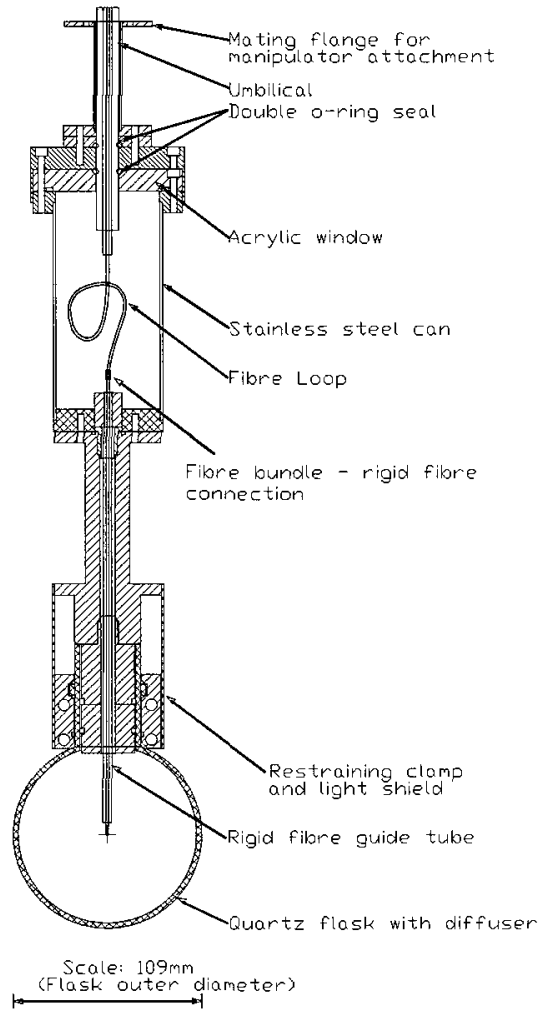

(b) Laserball.

Figure 2.4: Schematics of (a) the SNO source manipulator system and (b) laserball source [55]. The diffuser of the laserball source used for PMT and optical calibrations has an outer radius of $R_{\mathrm{LB}}=5.45 \mathrm{~cm}$.

PMTs per event. The light guide is contained within a specially-made cable for SNO calibrations, called the umbilical [56], which is also partly responsible for holding the source. Figure $2.4 \mathrm{~b}$ shows a schematic of the LB depicting the umbilical attachment at the top, and the fiber positioned at the center of the diffuser. The light scatters and propagates through the flask medium to come out almost isotropically at the surface. Hence the LB provides an excellent source of photons for calibrations. The PMT times (PCA), media attenuation lengths, PMT angular response, PMT relative efficiencies, and LB isotropy are all extracted from LB data. Technical details on the 


\begin{tabular}{|l|c|c|c|c|c|c|}
\hline \hline & \multicolumn{6}{|c|}{ Wavelength (nm) } \\
\hline Nominal & 337.1 & 369 & 386 & 420 & 500 & 620 \\
Spread & 0.1 & 10 & 8 & 8 & 14 & 10 \\
\hline
\end{tabular}

Table 2.2: Wavelengths available for optical calibrations. PMT timing calibration (PCA) was performed at $500 \mathrm{~nm}$ only.

laser system, umbilical, and LB can be found in [55].

\section{Energy, Electron, Neutron, and Other Calibrations}

The ${ }^{16} \mathrm{~N}$ triggered source $[57,58]$ is the main calibration source deployed to measure the overall collection efficiency, energy systematic uncertainties such as position-toposition uncertainties, time variations in energy, and accuracy of the position vertex fitter. It is a $6.13-\mathrm{MeV} \gamma$-ray source, emitted by the de-excitation of the ${ }^{16} \mathrm{O}$ resulting from the $\beta$-decay of ${ }^{16} \mathrm{~N}$. The Compton scattering effect allows the ${ }^{16} \mathrm{~N}$ source to be treated as an electron source.

The ${ }^{8} \mathrm{Li}$ source [59] is a triggered low-rate electron source with an endpoint of $14 \mathrm{MeV}$ obtained from the $\beta$-decay of ${ }^{8} \mathrm{Li}$. As a low statistics source, it serves to confirm and extrapolate of the energy systematic uncertainties derived from the ${ }^{16} \mathrm{~N}$ source at higher energy, as well as to determine possible energy scale non-linearities.

The $\mathrm{pT}$ source [60] is a non-triggered source that collides protons on a ${ }^{3} \mathrm{H}$ target producing ${ }^{4} \mathrm{He}$ which through de-excitation produces a $19.8-\mathrm{MeV} \gamma$. This source was not deployed in the salt phase due to overwhelming production of neutrons. It was mainly used to determine the energy scale non-linearities at high energy.

The ${ }^{252} \mathrm{Cf}$ source is a non-triggered neutron source obtained from the fission of ${ }^{252} \mathrm{Cf}$. It is the main source deployed to determine the neutron capture efficiency in 
SNO [61] in all three phases.

The AmBe source is a manually-triggered source with a $4.14 \mathrm{MeV} \gamma$-ray and neutron in coincidence. It is triggered by requiring the latter coincidence. It was used to verify the neutron capture efficiency. This source was not deployed during the $\mathrm{D}_{2} \mathrm{O}$ phase.

The radioactive and acrylic sources [62] are either ${ }^{238} \mathrm{U}$ or ${ }^{232} \mathrm{Th}$ sources encapsulated in acrylic, used to measure the detector's acceptance to low energy backgrounds. These sources can either be canned (acrylic is surrounded by plastic which prevents $\beta$ 's from producing light in the detector) or uncanned. None of these sources can be triggered easily. However, they were made very hot to overwhelm the nominal amounts of radioactivity.

The radon spike [63] is a controlled injection of $\mathrm{Rn}$ in the detector either into the $\mathrm{D}_{2} \mathrm{O}$ or $\mathrm{H}_{2} \mathrm{O}$ through the water systems. It was a highly isotropic source used to measure the detector response to low energy backgrounds. Similar to other radioactive sources, the source is not triggered but overwhelms the low inherent radioactivity within the detector.

\subsection{NCD System}

The NCD system was operational only during the NCD phase of the SNO experiment. The system, consisting of 40 counters and associated electronics, added 40 independent channels to the experiment. Subsection 2.3.1 focuses on the NCD trigger system. Then Subsection 2.3 .2 briefly covers the calibration of the NCDs. Extensive details of the NCD electronics and calibrations can be found in [52]. Figure 2.3 showed a NCD and its geometrical characteristics. 


\subsubsection{Electronic and Trigger Systems}

The NCD electronics were designed with several goals: perform pulse-shape discrimination to distinguish neutron-capture signals from $\alpha$-particle backgrounds and instrumental events, measure of the total charge of the events, and acquire the information at the $\mathrm{kHz}$ rates in the event of a galactic supernova. These goals were satisfied with two independently-triggered readout systems. The first is a fast data path using shaper-ADCs to measure the total energy of the signal. The second is a multiplexed digitizing path to record the pulse shape information for particle identification.

\section{Preamplifiers and Multiplexers}

Current preamplifiers were needed to amplify the NCD signal currents to voltages $(27.5 \mathrm{mV} / \mu \mathrm{A})$ before digitization. The preamplifiers were located above the top of the AV neck on the deck, minimizing their distance from the NCD strings. Their output cables were connected to one of the multiplexer input channels located in NCD electronics racks.

The four multiplexer boxes each had 12 channels connected to two parallel buffer amplifiers, reaching the shaper-ADC system on one side and the digitizing system consisting of two oscilloscopes on the other. Logarithmic amplifiers digitized the signals exceeding the discriminator thresholds. The 12 channels shared a single logarithmic amplifier output, so simultaneous events on different channels were read out as a sum of the signals on all channels above their discriminator levels. The information on which particular multiplexer box had triggered was sent to the multiplexer controller for each digitized event so that the event could be correlated with the appropriate NCD string. The DAQ system then received the channel hit pattern for each mul- 
tiplexer box. The average event rate of $0.3 \mathrm{~Hz}$ was low enough such that events associated with multiple channels were rare. The associated read-out dead time was approximately $1 \mathrm{~ms}$.

\section{Oscilloscopes and Digitization}

Upon receiving notification of a multiplexer channel exceeding its discriminator, the multiplexer control board triggered one of two Tektronix TDS754A oscilloscopes to digitize the signal waveform from the logarithmic amplifier. Only the signal from the appropriate boxes was read out by the DAQ when notified by the multiplexer controller which boxes and oscilloscopes contained the event. The sample rate of the digitization at the oscilloscopes was $1 \mathrm{GHz}$ to provide good vertical position resolution and pulse-shape discrimination. The multiplexer controller could only trigger one of the four available channels on the oscilloscopes, allowing for a maximum waveform digitization rate of $1.8 \mathrm{~Hz}$, adequate for the low neutrino data-taking rates.

\section{Shapers and Energy}

Due to the long digitization time of the waveforms, the NCD signals were also sent from the multiplexer to the input of shaper-ADC cards, each containing eight channels. The shaper-ADCs were capable of recording the $\mathrm{kHz}$ event rates expected from a supernova event burst. The signal was integrated and shaped by a four-stage network of operational amplifiers. The ADCs were calibrated to energy using the characteristic shape of the neutron energy spectrum and by determining the linear conversion of $\mathrm{ADC}$ values from the $191 \mathrm{keV}$ cut-off to the $764 \mathrm{keV}$ peak. Although each shaper channel was independent, the DAQ only allowed one shaper at the time to be read, 
which induced a dead time of $236 \pm 5 \mu \mathrm{s}$.

\section{Triggering with PMTs}

Signals from the independent DAQ hardware of the NCD and PMT arrays were integrated in a GT system that combined both data streams with timing information of the events. This was accomplished using the SNO Master Trigger Card (SNOMTC) that assigned a GT identification number (GTID) and recorded the times from both the 10- and 50-MHz clocks. The SNO-MTC was capable of triggering on a number of external inputs and two of them were used by the NCD trigger system. As explained above, the NCD system was triggered by the shaper-ADC or the multiplexer system whenever an event exceeded the threshold in either system. The NCD trigger system was controlled by a NCD Trigger Card (NCD-TC) containing a local GTID register that was synchronized with the SNO-MTC GTID register at all times. Thus, when a NCD event occurred, the SNO-MTC was informed and assigned a GTID, and this GTID was available for local readout in the NCD data stream. This allowed the NCD and PMT data to be combined based on the GTID of the event.

\subsubsection{Calibrations}

The following briefly describes the electronics and neutron calibrations needed to determine the properties and accuracy of the NCD system.

\section{Electronics and Energy}

The NCD-ECA [64] was performed on a weekly basis to quantify the parameters of an electronics model that described the logarithmic amplification and digitization of 
NCD waveforms, linear response of the system, and threshold of each shaper-ADC and digitization channel. These measurements were obtained by injecting waveforms into each preamplifier's pulser input and observing the output signal.

One set of calibrations measured the effects due to logarithmic amplification and waveform digitization. The parameters, that were extracted by fitting the sine-wave portion of the pulses, were then stored for pulse-shape data analysis. The electronics model parameters changed by less than $3 \%$ over the two-year duration of the NCD phase.

Another set was the linearity calibration that measured the gains and pedestals, and tested the linear response of the system. Each shaper-ADC channel was pulsed at a rate of $50 \mathrm{~Hz}$ for five seconds with $1.1-\mu \mathrm{s}$ wide, negative-polarity square waves at five different amplitudes from $36 \mathrm{mV}$ to $155 \mathrm{mV}$. In addition, an extended linearity calibration was performed, with 19 different amplitudes from $13 \mathrm{mV}$ to $248 \mathrm{mV}$, on a monthly basis. The gain and pedestal of each channel were obtained from a linear fit to the shaper-ADC values as a function of the injected pulse charge. The intercept from the linear equation and the peak position in the neutron calibration spectrum were then combined to convert shaper-ADC values to energies. The non-linearity is the difference between the measured and the linear fit charge value; it was found to be less than $0.5 \%$ over all channels.

Finally, the threshold calibration extracted the thresholds of each shaper-ADC and multiplexer channel as a function of the input pulse amplitude and charge. Offset, single-cycle sinusoidal waves $1 \mu \mathrm{s}$ in width were injected with amplitudes varying from $6 \mathrm{mV}$ to $31 \mathrm{mV}$, at a rate of $10 \mathrm{~Hz}$ for $5 \mathrm{~s}$ on each channel. The thresholds for each channel were determined by noting which pulses triggered both systems. The 
variation was less than $3 \%$ over the entire NCD phase. Uniform response across the $36{ }^{3} \mathrm{He}$ NCD strings is achieved with consistent threshold settings, particularly the multiplexer thresholds. Low thresholds were desirable to collect more neutron-capture events, however the final configuration included larger thresholds to avoid high noise trigger rates that caused large dead time in the oscilloscopes. The approximate digitization rate of $0.3 \mathrm{~Hz}$ caused the oscilloscopes to be dead about $3 \%$ of the time, which was accounted for in the livetime calculation. The multiplexer thresholds missed less than $2 \%$ of all neutron-capture events. This loss was incorporated into the neutron detection efficiency.

\section{Neutrons}

Extensive calibrations were carried out to characterize the response of the NCDs to neutrons. The ${ }^{252} \mathrm{Cf}$ and $\mathrm{AmBe}$ point-sources described in Subsection 2.2.2 were used for these calibrations. The source positions were varied using the calibration system to expose the sub-counters near the accessible axes and verify the neutron event rate and position in each string [65].

An isotropic source of neutrons, produced by mixing ${ }^{24} \mathrm{Na}[66]\left(\tau_{1 / 2}=14.959\right.$ hours) in the $\mathrm{D}_{2} \mathrm{O}$, was used twice to calibrate the response of the NCD system. Neutrons are produced by deuterium photo-disintegration induced by the release of $2.754-\mathrm{MeV} \gamma$ 's from the decay of ${ }^{24} \mathrm{Na}$. The uniformity of this neutron source approximately reproduces the expected $\mathrm{NC}$ interaction distribution of neutrons in the $\mathrm{D}_{2} \mathrm{O}$. However, this calibration was lengthy because it required at least two weeks before the ${ }^{24} \mathrm{Na}$ activity completely decayed away. The efficiency of the NCD array to capture the uniformly distributed neutrons has been measured with this technique 
to be $(21.1 \pm 0.7) \%$ [27]. In addition, these calibrations were used to track the gain of each NCD string over time with the position of the $764-\mathrm{keV}$ neutron-capture peak.

\subsection{Backgrounds and Water Systems}

The primary goal of the water purification system was to control the level of impurities in the $\mathrm{D}_{2} \mathrm{O}$ and $\mathrm{H}_{2} \mathrm{O}$ to consequently reduce the backgrounds to the low energy neutrino events. This section introduces the different types of backgrounds that are expected in SNO, and the main functionalities of the water systems to measure and control a few of them.

\subsubsection{Low Energy Backgrounds}

This subsection describes the known sources of backgrounds that are the most significant and relevant in the context of a low neutrino energy analysis. In such analysis, all sources of background from a reconstructed energy of $3.5 \mathrm{MeV}$ need to be considered. These backgrounds are measured, constrained, and fitted simultaneously with the neutrino signals in the signal extraction procedure that is explained in Chapter 5.

There are two classes of backgrounds in SNO, defined with their origin with respect to the fiducial $\mathrm{D}_{2} \mathrm{O}$ volume: external or internal. External backgrounds come from radioactivity in the AV, light water, PSUP, and PMT glass and reflectors. Events that result from these backgrounds have distinct radial distributions. Internal backgrounds arise from extremely small traces of radioactivity left in the $\mathrm{D}_{2} \mathrm{O}$ and NCDs and cannot be subtracted from neutrino events other than by measuring their concentration in the detector. 
Those two classes then break into two sub-classes of contamination. The radioactive elements responsible for neutron backgrounds can release energy in the form of $\gamma$-rays with energies above $2.224 \mathrm{MeV}$, the threshold of the NC reaction, and disintegrate deuterons to create neutrons. These $\gamma$ 's can also Compton-scatter and initiate Čerenkov background events. Similarly, the elements responsible for Čerenkov backgrounds release charged $\beta$ 's that can initiate the creation of C light. The sub-classes are treated separately below but are in practice hard to distinguish from one another.

Figure 2.5 shows the dominant decay chains of ${ }^{238} \mathrm{U}$ and ${ }^{232} \mathrm{Th}$ and their respective daughters. These decay chains are responsible for the bulk of the neutron- and electron-like backgrounds discussed below.

\section{Neutron Backgrounds}

The energies of neutron events in SNO are uncorrelated with the incoming neutrino energies. While extracting the number of neutrons due to neutrinos it is therefore crucial to take into account all sources of neutron backgrounds.

Figure 2.5a shows the decay chain of ${ }^{238} \mathrm{U}$ leading to the ${ }^{214} \mathrm{Bi}$ daughter that decays with an endpoint energy of $3.27 \mathrm{MeV}$. It also decays $2.8 \%$ of the time to an excited state of ${ }^{214} \mathrm{Po}$ that emits a $\gamma$-ray of $2.445 \mathrm{MeV}$. This energy can initiate the deuteron photo-disintegration and produce at most one background neutron per decay chain.

Figure $2.5 \mathrm{~b}$ shows the decay chain of ${ }^{232} \mathrm{Th}$ producing the ${ }^{208} \mathrm{Tl}$ daughter that decays to an excited state of ${ }^{208} \mathrm{~Pb}$ through several $\beta$ branches. The de-excitation of ${ }^{208} \mathrm{~Pb}^{*}$ always emits $2.614 \mathrm{MeV} \gamma$ 's which can also photo-disintegrate the deuteron and produce a background neutron. Both ${ }^{214} \mathrm{Bi}$ and ${ }^{208} \mathrm{Tl}$ can at most generate one neutron each per decay chain. Therefore the measurement of their concentrations in 


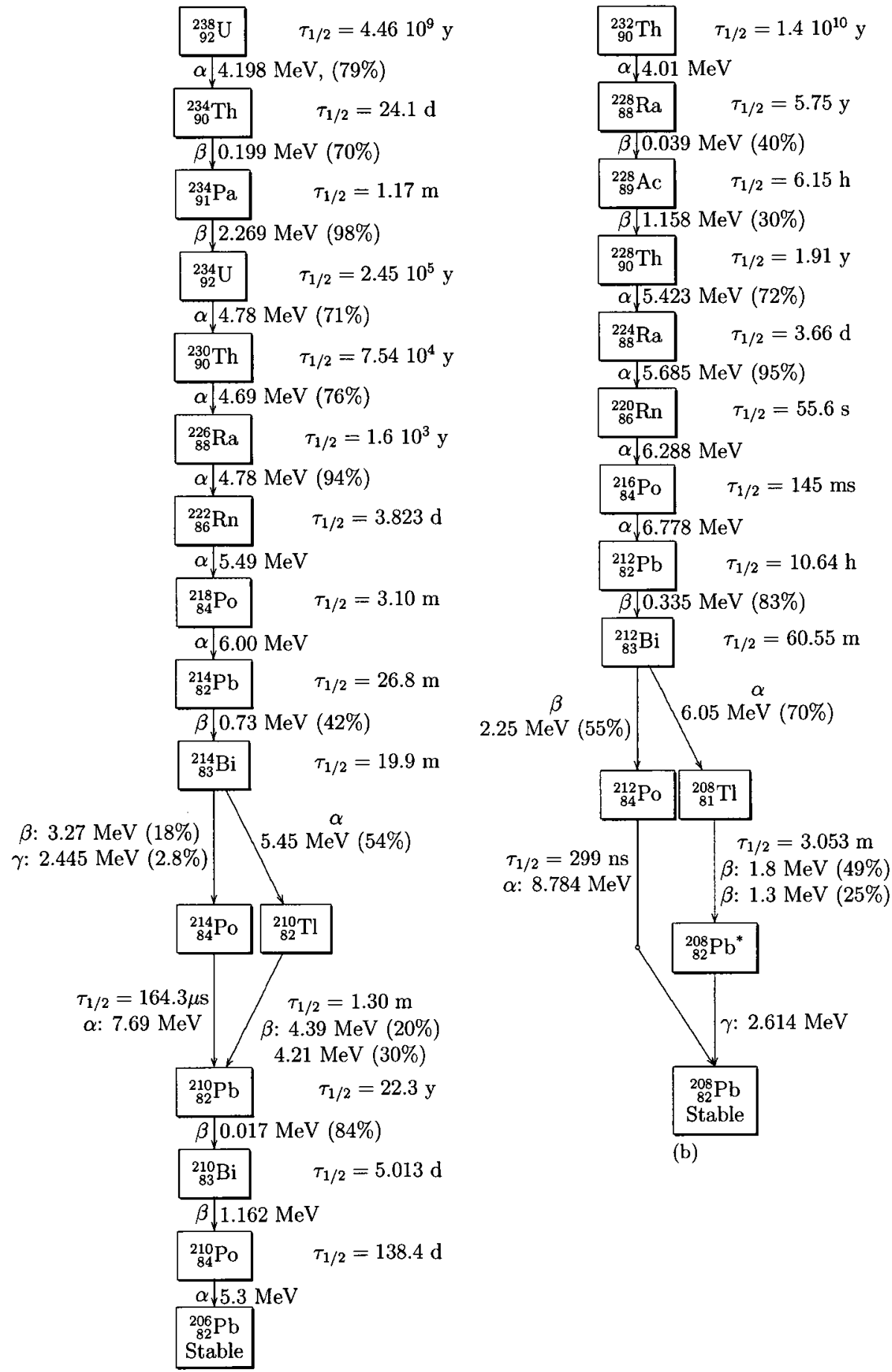

(a)

Figure 2.5: Radioactive decay chains of (a) ${ }^{238} \mathrm{U}$ and (b) ${ }^{232} \mathrm{Th}$. The steps in each chain are labeled with half-life, $\tau_{1 / 2}$, probability in percent, particles emitted, and released energy. 
the $\mathrm{D}_{2} \mathrm{O}$ and $\mathrm{H}_{2} \mathrm{O}$ is important for the extraction of the total neutrino flux.

External neutrons are produced by similar processes but originate from outside the $\mathrm{D}_{2} \mathrm{O}$ volume, where contaminants are more present. Additionally, the processes that create $\alpha$ 's in Figure 2.5 can potentially produce neutrons through $(\alpha, n)$ reactions in the AV. However the rate of these reactions are negligible compared to the AV neutrons, created from impurities that deposited on the AV during the detector construction. For instance, radon from the mine air, ${ }^{222} \mathrm{Rn}$, produces daughters that emit $\alpha$ 's that can interact directly with carbon in the AV and release a free neutron.

In the salt phase only, another source of background neutrons arise from ${ }^{24} \mathrm{Na}$. Natural sodium, in the form of ${ }^{23} \mathrm{Na}$, is activated by neutrons in the environment to form ${ }^{24} \mathrm{Na}$. The activation occurs after a source of neutrons has been deployed for calibrations. The decay chain of ${ }^{24} \mathrm{Na}$ leads to the emission of a $2.754-\mathrm{MeV} \gamma$-ray $99.9 \%$ of the time.

\section{Čerenkov Backgrounds}

Cerenkov backgrounds are caused by $\beta$ 's with kinetic energy $\gtrsim 265 \mathrm{keV}$ creating $\check{\mathrm{C}}$ light directly or indirectly by Compton-scattering of $\gamma^{\prime}$ s and electrons. With an analysis threshold of $3.5 \mathrm{MeV}$, both decay chains in Figure 2.5 contribute to those backgrounds. The same sources responsible for neutron backgrounds, the $3.27-\mathrm{MeV}$ $\beta$ from ${ }^{214} \mathrm{Bi}$ and the $2.614-\mathrm{MeV} \gamma$ from ${ }^{208} \mathrm{Tl}$, can also initiate $\check{\mathrm{C}}$ radiation.

In the analysis, these backgrounds have similar features as neutron backgrounds. Internal sources are indistinguishable and must be measured and carefully compared with Monte Carlo simulations, and external sources can be separated from the signal using the reconstructed positions of the events. 


\section{Other Backgrounds}

The PMT backgrounds $\left(\mathrm{PMT}_{\gamma}^{\beta}\right)$ are defined as the sum of the $\beta-\gamma$ backgrounds coming from $\mathrm{U}$ and $\mathrm{Th}$ in the PSUP, PMT glass, internal components, and reflectors. The orientation of the reflector provides natural shielding against these backgrounds, but many events are still detected between 3.5 and $4 \mathrm{MeV}$. The proportion of these events dominate the total number of events at the analysis threshold of $3.5 \mathrm{MeV}$.

Leslie events ${ }^{1}$ are a small class of background originating near the AV, characterized by an apparent high degree of isotropy and a wide range of energy values. The mechanism generating these events remains unknown, but they often are misreconstructed into the $\mathrm{D}_{2} \mathrm{O}$ volume leading into potential contamination. These events are usually removed from the analysis samples by the means of MC studies.

Counts due to sources of backgrounds from outside the detector, such as atmospheric neutrinos, cosmogenic neutrons, and anti-neutrinos, are estimated and subtracted using the expected fluxes and detector livetime. Others such as muons and high-energy particles are tagged by OWL tubes and are clearly identified by their large number of hit PMTs.

\subsubsection{Background Measurement Techniques}

Control of the contaminants in SNO is the hardest task of water operations due to the constant exposure of the detector with mine air, equipment, calibration devices, and even personnel entering the laboratory. Recirculation of both $\mathrm{D}_{2} \mathrm{O}$ and $\mathrm{H}_{2} \mathrm{O}$ is performed periodically to remove as much $\mathrm{U}$ and $\mathrm{Th}$ as possible. The liquids are circulated through pipes out and back into the detector. The water is filtered,

\footnotetext{
${ }^{1}$ After Hamish Leslie, a SNO collaborator who was among the first to notice their existence.
} 
de-ionized, de-gassed to remove radioactive components, and re-gassed with pure nitrogen. Although this technique keeps the level of impurities to a low level for

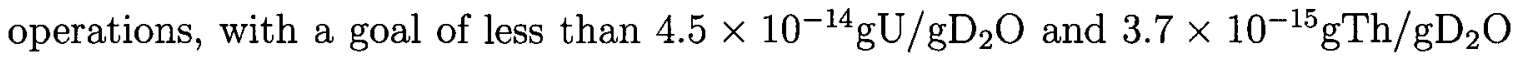
in $\mathrm{D}_{2} \mathrm{O}$, it is desirable to measure these quantities. The determination of the amount of radioactive components in SNO is crucial to all analyses, specifically to remove events due to internal backgrounds.

The in situ methods, preferred in this thesis, were developed to evaluate the number of events in the neutrino data set using analysis windows that are not used in the neutrino data analysis, such as radial positions outside the AV. The extrapolation back into the analysis window allowed the background events to be subtracted. Such method can also extract the neutrino signal and background events simultaneously in a single analysis window. In order to do that, constraints of the background rates are needed from independent measurements known as ex situ techniques. There are two ex situ techniques to assay the water from the detector to determine the $U$ and Th levels. They are briefly described here.

\section{Ex Situ Techniques}

The first technique is called $\mathrm{HTiO}$ [67], which stands for the chemical compound used as an ion-exchanger to collect the radioactive components present in water. The compound is deposited on microfiltration membranes within columns which are placed underground in large volumes of $\mathrm{D}_{2} \mathrm{O}$ and $\mathrm{H}_{2} \mathrm{O}$ above the $\mathrm{SNO}$ detector. The result is then eluted from the membranes with acid, concentrated, and finally mixed with a scintillator to count the $\beta-\alpha$ decays in coincidence. The activity from the Th chain is obtained from the activity levels of ${ }^{228} \mathrm{Th},{ }^{224} \mathrm{Ra}$, ${ }^{212} \mathrm{~Pb}$, and ${ }^{212} \mathrm{Bi}$. Similarly for the $\mathrm{U}$ 


\begin{tabular}{|c|c|c|}
\hline \hline Phase & Background & Measurement $\left(\times 10^{-14}\right)$ \\
\hline \hline \multirow{5}{*}{$\mathrm{D}_{2} \mathrm{O}$} & External Bi & $(29.5 \pm 5.1) \mathrm{gU} / \mathrm{gH}_{2} \mathrm{O}$ \\
& External Tl & $\left(8.1_{-2.3}^{+2.7}\right) \mathrm{gTh} / \mathrm{gH}_{2} \mathrm{O}$ \\
& Internal $\mathrm{Bi}$ & $\left(1.01_{-0.20}^{+0.34}\right) \mathrm{gU} / \mathrm{gD}_{2} \mathrm{O}$ \\
& Internal Tl & $\left(0.209 \pm 0.021_{-0.091}^{+0.096}\right) \mathrm{gTh} / \mathrm{gD}_{2} \mathrm{O}$ \\
\hline \multirow{5}{*}{$\mathrm{Salt}$} & External Bi & $(20.6 \pm 5.0) \mathrm{gU} / \mathrm{gH}_{2} \mathrm{O}$ \\
& External Tl & $(5.2 \pm 1.6) \mathrm{gTh} / \mathrm{gH}_{2} \mathrm{O}$ \\
& Internal Bi & $<2.0 \mathrm{gU} / \mathrm{gD}_{2} \mathrm{O}$ \\
& & $>(1.41 \pm 0.46) \times 10^{-2} \mathrm{gU} / \mathrm{gD}_{2} \mathrm{O}$ \\
& Internal Tl & $\left(0.176 \pm 0.044_{-0.04}^{+0.07}\right) \mathrm{gTh} / \mathrm{gD}_{2} \mathrm{O}$ \\
\hline \multirow{5}{*}{$\mathrm{NCD}$} & External Bi & $\left(35.5_{-5.4}^{+9.9}\right) \mathrm{gU} / \mathrm{gH}_{2} \mathrm{O}$ \\
& External Tl & $(2.77 \pm 1.04) \mathrm{gTh} / \mathrm{gH}_{2} \mathrm{O}$ \\
& Internal Bi & $(0.614 \pm 0.101) \mathrm{gU} / \mathrm{gD}_{2} \mathrm{O}$ \\
& Internal Tl & $(0.077 \pm 0.021) \mathrm{gTh} / \mathrm{gD}_{2} \mathrm{O}$ \\
\hline \hline
\end{tabular}

Table 2.3: Main sources of backgrounds in SNO [26, 30,70]. The main elements are associated with the $e x$ situ measurements of $\mathrm{U}$ and $\mathrm{Th}$. When two uncertainties are given the first one is statistical and the second is systematic.

chain, the activity is obtained from the activity levels of ${ }^{226} \mathrm{Ra},{ }^{222} \mathrm{Rn},{ }^{214} \mathrm{~Pb}$, and ${ }^{214} \mathrm{Bi}$.

The second technique is called $\mathrm{MnOx}$ [68]. It stands for the coating of acrylic beads made of a manganese oxide compound, $\mathrm{MnO}_{x}$, where $x$ indicates a variable amount of oxygen. After water flows through columns containing the coated beads, $\mathrm{Ra}$, predominantly, is removed, dried, and placed in a decay chamber where Po ions are detected using an $\alpha$-counter.

Both methods are compared before the estimates of the concentrations of radioactive elements are distributed to the analysts. Table 2.3 is a summary of the concentration measurements of $\mathrm{U}$ and Th performed in all phases of SNO that are were inputted in the analysis in this thesis. Technical details about the water systems and the ex situ extraction methods can be found in [67-69]. 


\subsection{Detector Simulation}

The processing of PMT and NCD information from the SNO electronics and the Monte Carlo (MC) simulation are handled by the SNO Monte Carlo and ANalysis software (SNOMAN) [71], a package of FORTRAN routines built with the CERNLIB ZEBRA memory management data structure [72]. As a result of the multiple types of calibration, the detector constants are stored in data or command files that are applied to both data and MC processing streams consistently. The SNOMAN code enforces the relevant calibration constants by accessing the SNO database which is based upon the CERNLIB HEPDB software package [73]. SNOMAN also contains the position and direction reconstruction as well as energy reconstruction algorithms that estimate the location, direction, and energy of both data and MC events.

In the MC simulation, the propagation of electrons and $\gamma$ 's is handled by the Electron Gamma Shower simulation code (EGS4) [74] called internally by SNOMAN. The number of Č photons emitted along a charged particle's track is determined from the asymptotic formula for light yield for each track segment in EGS4 [75]. Neutron propagation and capture processes, up to an energy of $20 \mathrm{MeV}$, are modeled using the Monte Carlo N-Particle Transport code system (MCNP) [76]. Particles of energies of $2 \mathrm{GeV}$ and above are simulated with LEPTO [77] (muons and electrons) and FLUKA [78] (hadrons) available in CERNLIB. Additional modules can be appended to SNOMAN for specific simulations; see [34] for example. Therefore SNOMAN is capable of simulating all relevant interactions in SNO including neutrino interactions, electrons, $\gamma$ 's, neutrons, all the calibration sources described in Subsections 2.2.2 and 2.3.2, low energy decays from the $\mathrm{U}$ and $\mathrm{Th}$ chains, and atmospheric and cosmic 
muons. Additionally, the simulation framework models the full detector geometry, such as the AV, acrylic tiles, acrylic belly plates and grooves, kevlar ropes, neck, NCD array with anchors and cables, PSUP and calibration source containers. Finally, a detailed simulation of neutron and $\alpha$ detection has been implemented in SNOMAN and tested vigorously for the NCD phase [79]. The geometry was updated to allow NCD positions, sizes, readout cables, and other relevant parameters to be changed for the study of systematic uncertainties [65].

\section{Importance of Calibrations and Simulations}

The good performance of SNOMAN in neutrino and background event simulations relies in great part on the PMT simulation. The angular response of the PMTs, or the efficiency of the PMTs as a function of the photon's angle of incidence and wavelength, is modeled in two ways. The first is a detailed simulation of photon propagation in a three-dimensional representation of the phototube (3D-PMT) [75]. This model propagates individual photons through a PMT and concentrator, simulating the photo-cathode response and reflections from the components. The 3D-PMT model was used in the official SNO MC simulation. A second model, called grey disk (GD), is an effective model of the PMT angular response. The GD model replaces the opening surface of the PMT by a disk with the probability of detecting photons as a function of incident angle and wavelength derived directly from laserball measurements. The PMT angular response in these two models agree well [80], therefore the SNOMAN PMT response 3D-PMT model is adjusted to the measurements obtained from optical calibrations.

The following two chapters focus on the analysis of calibration data to obtain 
an accurate detector response used as input to the full Monte Carlo simulation and data processing. Chapter 3 introduces the optical calibration methods and the upgrades performed during the NCD commissioning phase. These upgrades led to the characterization of the average PMT response and individual PMT relative efficiencies. These measurements served, first, in the new optical calibration methods of the NCD phase, detailed in Chapter 4, and second, in the low energy threshold analysis to improve the detector simulation, outlined in Chapter 5. Without loss of generality, Chapters 3 and 4 can then be skipped by the reader strictly interested by the physics of neutrinos. Chapter 5 resumes with the signal extraction procedure developed specifically in the context of the low energy threshold analysis, leading to the description of the survival probability fit in Chapter 6, and the physics interpretation of the results in terms of oscillation parameters in Chapter 7. 


\section{CHAPTER 3}

\section{Optical Calibration in the NCD Commissioning Phase}

The present and next chapters are a break from the main discussion to introduce the work done on the optical calibration (OCA) of the SNO detector after the salt phase. The upgrades explained in this chapter were useful in two SNO analyses. First, the analysis of the NCD OCA data (Chapter 4), and second, the re-analysis of the $\mathrm{D}_{2} \mathrm{O}$ and salt phases data (Chapter 5 and onwards). These upgrades arise from the continuation of studies previously done for the $\mathrm{D}_{2} \mathrm{O}$ [56] and salt $[81,82]$ phases. Particularly, this chapter explains the optical model and the PMT efficiency extraction method using the OCA data taken before the NCD phase, and Chapter 4 presents a complete description of the improvements and changes needed towards the NCD phase, with the results and evaluation of systematic uncertainties.

\subsection{Introduction}

The NCD commissioning phase consisted in the installation of the NCDs into the SNO detector after desalination, and of their preparation for data taking. That period started in October 2003 and ended in August 2004, during which two OCA 
scans were performed by positioning the laserball (LB) in many places in the detector at the six different wavelengths given in Table 2.2.

The October/03 scan was necessary to verify the detector optics after the salt was removed. It was confirmed that the detector optical properties were identical to the $\mathrm{D}_{2} \mathrm{O}$ and salt phases at the time of the October/03 scan, which makes it the benchmarking calibration data set for comparisons with the NCD phase. Similarly, and often in this chapter, the September/00 scan, the first good quality scan in the $\mathrm{D}_{2} \mathrm{O}$ phase, is compared with the October/03 scan because of the similarities between the two detector configurations. The two scans were performed without salt in the detector, before and after the salt phase, respectively, and are referred to as $\mathrm{D}_{2} \mathrm{O}$ scans.

The July/04 scan proceeded to ensure that the PMT and NCD systems worked properly before the official start of the NCD phase. At this stage the NCD system was fully operational, and the PMT system had been upgraded with new electronic backplanes in an attempt of lowering the trigger thresholds during the NCD phase [83]. Unfortunately the quality of the data acquired during the July/04 scan was barely satisfactory, mostly due to the poor isotropy of the LB source at that time. The July/04 scan verified that all systems behaved as expected but was unusable to obtain the detector optical properties.

\section{PMT Efficiencies}

The SNO PMT relative efficiencies have been extracted before the NCD commissioning phase to monitor the stability of the detector throughout the salt phase [82], but have never been used directly in the analysis of neutrino and calibration data. The 
latter study suggested that the PMT efficiencies were stable in the time span of the experiment and independent of possible aging effects of the PMT glass and reflectors.

During the NCD commissioning phase, efforts were made to extend the PMT efficiency extraction method with the goal of using them in two different ways. First, for the re-analysis of the $\mathrm{D}_{2} \mathrm{O}$ and salt neutrino data sets, with low energy threshold, to improve the spatial variations of the detector response model when comparing the MC to the data. Second, for reasons explained in Section 3.2, for the processing of the OCA data in the NCD phase. The PMT efficiencies measured in the NCD commissioning phase were precious information that allowed the calibration precision to be maintained at the same level as previous phases of SNO.

Section 3.2 introduces the SNO optical model and the methods developed to extract its parameters. Section 3.3 explains how the PMT efficiencies were measured from the comparison of the OCA data to the model. Then Section 3.4 discusses the OCA method with input efficiencies and additional systematic uncertainties associated with them. Chapter 4 describes the NCD phase optical calibration of the detector. More details on the OCA and PMT efficiencies can be found in [84-86].

\subsection{Optical Calibration Concepts}

This section reviews the quantities of the SNO optical model established in [56] for the $\mathrm{D}_{2} \mathrm{O}$ and salt phases. 


\subsubsection{Optical Model}

The LB was the only source deployed in the detector for OCAs. The position of the LB and wavelength of the laser, $\lambda$, define a run with index $i$. The index 0 is reserved for runs in the center of the detector, central runs, where spherical symmetry applies.

The prompt intensity, $N_{i j}$, observed for run $i$ in PMT $j$ is parametrized as follows:

$$
N_{i j}=N_{i} \Omega_{i j} R_{i j} L_{i j} T_{i j} \epsilon_{i j} e^{-\left(d_{i j}^{\mathrm{d}} \alpha_{\mathrm{d}}+d_{i j}^{\mathrm{a}} \alpha_{\mathrm{a}}+d_{i j}^{\mathrm{h}} \alpha_{\mathrm{h}}\right)},
$$

where the multiplicative factors, described below, are either calculated from the detector geometry or extracted from the LB data. The total number of parameters in the model is larger than the number of multiplicative factors because each term hides dependences on geometric variables such as angles of incidence. The model can contain up to 500 free parameters that the LB data must constrain:

$N_{i}$ Number of photons emitted by the LB in run $i$ and detected within the \pm 4 ns prompt time window of each PMT. This term is the intensity normalization for each run and cannot be precisely measured. There is one such parameter per run and typically between 25 and 50 runs per wavelength per scan.

$\Omega_{i j}$ Solid angle or the acceptance in run $i$ for the $\operatorname{PMT} j$. This term is calculated based on the detector geometry.

$R_{i j}$ Additional geometric correction factors which describe the phototube and reflector angular response (also referred to as PMTR) beyond $\Omega_{i j}$. The main effects are the light transmission of the PMT glass material and the reflectivity of the concentrators. This factor is parametrized as a function of the incident 
angle of light on the PMT surface, $\theta_{\mathrm{PMT}}$, and is extracted from the data. For bins that are $1^{\circ}$-wide, there are as much as 45 independent parameters in $R_{i j}$.

$L_{i j}$ LB light distribution expressed as a function of angles relative to its position. $L_{i j} \equiv L_{i j}\left(\theta_{\mathrm{LB}}, \phi_{\mathrm{LB}}\right)$ is built from the product of the carriage mask and anisotropy functions. It is extracted from the data. There can be between 52 and 438 parameters depending on the internal parameterization.

$T_{i j}$ Fresnel transmission coefficients for the $\mathrm{D}_{2} \mathrm{O} / \mathrm{AV} / \mathrm{H}_{2} \mathrm{O}$ interfaces. These terms are calculated analytically, given the LB and PMT positions.

$\epsilon_{i j}$ Absolute quantum efficiency in run $i$ for the $\mathrm{PMT} j$ characterizing the wavelengthdependent probability of registering a hit. In principle each physical PMT has its own characteristic efficiency $\epsilon_{j}$, but $\epsilon_{i j}$ hides a dependence on quantities such as $\theta_{\mathrm{PMT}}$. The average values $\epsilon_{j}$ are extracted in this chapter.

$d_{i j}^{\mathrm{d}, \mathrm{a}, \mathrm{h}}$ Light path lengths through the $\mathrm{D}_{2} \mathrm{O}(\mathrm{d}), \mathrm{AV}(\mathrm{a})$ and $\mathrm{H}_{2} \mathrm{O}(\mathrm{h})$, respectively. These distances are calculated based on the detector geometry.

$\alpha_{d, a, h}$ Attenuation coefficients for the $\mathrm{D}_{2} \mathrm{O}(\mathrm{d}), \mathrm{AV}$ (a) and $\mathrm{H}_{2} \mathrm{O}(\mathrm{h})$, respectively. These coefficients are extracted from the data. The values of $\alpha_{\mathrm{a}}$ were fixed to the measurements reported in [56]; thus there are two parameters to measure with the data per wavelength.

The model expectation $N_{i j}$ is an accurate prediction of the measured occupancy, $O_{i j}$, obtained almost directly from the number of counts relative to the known number of laser pulses. Diffusion, reflection, and absorption are not modeled, such that detector components (ropes, thick AV support plates, etc.) standing in the optical path between the LB and a PMT leads to the rejection of the PMT $[56,87]$. Otherwise 
the PMT is labeled as good and keeps accumulating statistics by being in a good state in many runs.

The main goal of OCAs is to extract the water attenuation coefficients, $\alpha_{\mathrm{d}, \mathrm{h}}$, and PMTR $R\left(\theta_{\mathrm{PMT}}\right)$ for each wavelength. Subsection 3.2.2 introduces the OccupancyRatio method used to analyze LB data until the NCD commissioning phase. The method makes use of central runs to normalize all data and extract the model parameters. Subsection 3.2.3 explains how the analysis was modified to make use of a set of measured PMT efficiencies to obtain the same parameters.

\subsubsection{Occupancy-Ratio Method}

The Occupancy-Ratio (OccRatio) method transforms the data to ratios of PMT occupancies such that the numerous unknown PMT efficiencies cancel. The method strongly relies on high-statistics central runs to normalize all other runs so that the optical parameters can be obtained relative to the center of the detector.

In the OccRatio method, the optical model quantities are re-organized as follows. The solid angles $\Omega_{i j}$ and the transmission coefficients $T_{i j}$ terms of Equation (3.1) are directly calculated from the geometry of the detector given the LB and PMT positions. The data are corrected with factors $C_{i j}$, proportional to the geometric factors of the normalization run. The measured occupancy ratio, $O R_{i j}^{\text {data }}$, is:

$$
O R_{i j}^{\text {data }}=\frac{O_{i j}}{O_{0 j}} \times C_{i j}=\frac{O_{i j}}{O_{0 j}}\left(\frac{\Omega_{0 j} T_{0 j}}{\Omega_{i j} T_{i j}}\right),
$$

where $i=0$ refers to the central normalization run. The model occupancy ratio is 
then:

$$
O R_{i j}^{\text {model }}=\frac{N_{i j}}{N_{0 j}} \times C_{i j}=\frac{N_{i}}{N_{0}}\left(\frac{R_{i j} L_{i j}}{R_{0 j} L_{0 j}}\right) e^{-\left(\delta d_{i j}^{\mathrm{d}} \alpha_{\mathrm{d}}+\delta d_{i j}^{\mathrm{a}} \alpha_{\mathrm{a}}+\delta d_{i j}^{\mathrm{h}} \alpha_{\mathrm{h}}\right)},
$$

where $\delta d_{i j}=d_{i j}-d_{0 j}$ are the path differences in the three media. The distance $d_{i j}$ $\left(d_{0 j}\right)$ is calculated from the off-center (central) run source position to the $\operatorname{PMT} j$. The comparison of Equations (3.2) and (3.3) allows one to fit the unknown parameters: the run normalizations $N_{i}$, PMT response $R_{i j}$, LB light distribution $L_{i j}$, and media attenuation coefficients $\alpha_{\mathrm{d}, \mathrm{h}}$. The best-fit parameters are obtained by minimizing the $\chi^{2}$ differences for all runs and PMTs in a scan, with total statistics $N_{\text {run }} \times N_{\text {PMT }}$ :

$$
\chi^{2}=\sum_{i}^{N_{\mathrm{run}}} \sum_{j}^{N_{\mathrm{PMT}}} \frac{\left({ }_{j}^{\text {data }}-O R_{i j}^{\text {model }}\right)^{2}}{\left(\Delta Q R_{i j}\right)^{2}+v_{i j}^{2}}
$$

where $\Delta Q R_{i j}$ is the statistical uncertainty in the occupancy ratio due to counting statistics and $v_{i j}$ is an additional systematic uncertainty introduced to account for the PMT response variability as a function of $\theta_{\mathrm{PMT}}$, as explained in more details in Subsection 3.3.3.

\section{Statistics Problems in the NCD Phase}

The OccRatio method used to extract the optical constants during the $\mathrm{D}_{2} \mathrm{O}$ and salt phases relies strongly on the quality of the normalization run but more generally on pairs of runs. In the NCD phase, NCDs are expected to produce shadowing patterns in front of the PMTs. About $50 \%$ of the PMTs per run should be affected by partial shadowing, flagged as shadowed, and removed from the fit. The central run is subject to those patterns as much as any other off-center run. This means the occupancy ratio variable is subject to the convolution of two different shadowing patterns. This 
selection of ratios leads to an important decrease in the statistics of the fit [88]. The extraction of the numerous optical parameters with low statistics have direct effects on the accuracy of the fit, namely on $\alpha_{\mathrm{d}, \mathrm{h}}$ and the PMTR at high incident angles [89]. An alternative to the OccRatio fit is needed to avoid extending the calibration time unnecessarily or take data that would not be fully analyzed. To avoid the statistics loss, the optical model fit needs to be performed with the PMT occupancies directly, which means the efficiencies $\epsilon_{i j}$ in Equation (3.1) are needed as input.

\section{PMT Efficiency Estimator}

The efficiencies are not fitted directly as part of the model because it would require roughly 9,000 more parameters to be fitted independently. After the OccRatio fit has converged though, the optical model of Equation (3.1) predicts the occupancy of a PMT, given the conditions of a run and best-fit parameters. The residual ratio, data/model, can be interpreted as the unknown efficiency of a given PMT and as the estimator of the PMT efficiency:

$$
\epsilon_{i j}=\left.\frac{O_{i j}}{N_{i j}}\right|_{\chi_{\min }^{2}} .
$$

The measurement of the PMT efficiency $\epsilon_{j}$ is obtained from the average of $\epsilon_{i j}$ over many runs. Section 3.3 explains the details of the PMT efficiency extraction method, but first Subsection 3.2.3 introduces the alternative fit method, that is then tested in Section 3.4 and employed in the NCD phase OCA analysis in Chapter 4. 


\subsubsection{Occupancy-Efficiency Method}

The alternative method is called the Occupancy-Efficiency fit, or simply the Occupancy method. The method needs the efficiencies instead of central runs to scale the normalizations $N_{i}$. This is possible if $\epsilon_{i j} \sim \epsilon_{j}$ in Equation (3.1) by using the efficiencies measured from a scan and applying them to another. The efficiency-weighted occupancy $N_{i j}$ is then directly comparable to the occupancy $O_{i j}$ and the fit is performed by minimizing the $\chi^{2}$ function:

$$
\chi^{2}=\sum_{i}^{N_{\text {run }}} \sum_{j}^{N_{\mathrm{PMT}}} \frac{\left(O_{i j}-N_{i j}\right)^{2}}{\left(\Delta O_{i j}\right)^{2}+v_{i j}^{2}} .
$$

The $v_{i j}$ term is representative of the error on the efficiency measurements as a function of $\theta_{\text {PMT }}$ [85]. The parametrization of these errors is explained in Subsection 3.3.3.

\subsection{Extraction of Relative PMT Efficiencies}

The usage of the PMT efficiencies in the neutrino data processing and MC simulation is a significant improvement compared to a model of the SNO detector where the response of all 9438 PMTs is identical. In addition, the Occupancy method in OCAs, used during the NCD phase, relies on the accurate determination of the PMT efficiencies. This section explains the steps leading to the PMT efficiencies and their associated uncertainties. 


\subsubsection{Normalization of Raw Efficiencies}

For each PMT of each run, a raw efficiency $\epsilon_{i j}$ is obtained using Equation (3.5). The following three extraction steps gather, average, and normalize the raw efficiencies such that each PMT gets its own relative efficiency $\epsilon_{j}$.

First, the run-average efficiencies $\bar{\epsilon}_{i}$ are calculated:

$$
\bar{\epsilon}_{i}=\frac{1}{N_{\mathrm{PMT}}^{i}} \sum_{j}^{N_{\mathrm{PMT}}^{i}} \epsilon_{i j},
$$

where $N_{\mathrm{PMT}}^{i}$ is the number of calibrated PMTs in run $i$. Then each raw efficiency $\epsilon_{i j}$ of each run $i$ is divided by the latter run-average to get the PMT average efficiency, $\left\langle\epsilon_{j}\right\rangle$, over all runs:

$$
\left\langle\epsilon_{j}\right\rangle=\frac{1}{N_{\text {run }}^{j}} \sum_{i}^{N_{\text {run }}^{j}} \frac{\epsilon_{i j}}{\bar{\epsilon}_{i}},
$$

where $N_{\text {run }}^{j}$ is the number of runs where the PMT $j$ was found in a good state. Finally, these efficiencies are forced to be distributed around one, leading to the normalized efficiency:

$$
\epsilon_{j}=\left\langle\epsilon_{j}\right\rangle / \frac{1}{N_{\mathrm{PMT}}} \sum_{j}^{N_{\mathrm{PMT}}}\left\langle\epsilon_{j}\right\rangle,
$$

where $N_{\mathrm{PMT}}$ is the number of physical PMTs kept until the latter step. The distribution of the efficiencies $\epsilon_{j}$ is a normal distribution centered around one by construction. Therefore the normalized efficiencies $\epsilon_{j}$ are referred to as relative efficiencies. Figure 3.1 shows the distributions of the raw and final relative efficiencies where both distributions are scaled by their maximum value. The above three steps bring the mean at one, as wanted, but also reduce the spread of the distribution. Subsec- 
PMT Efficiencies

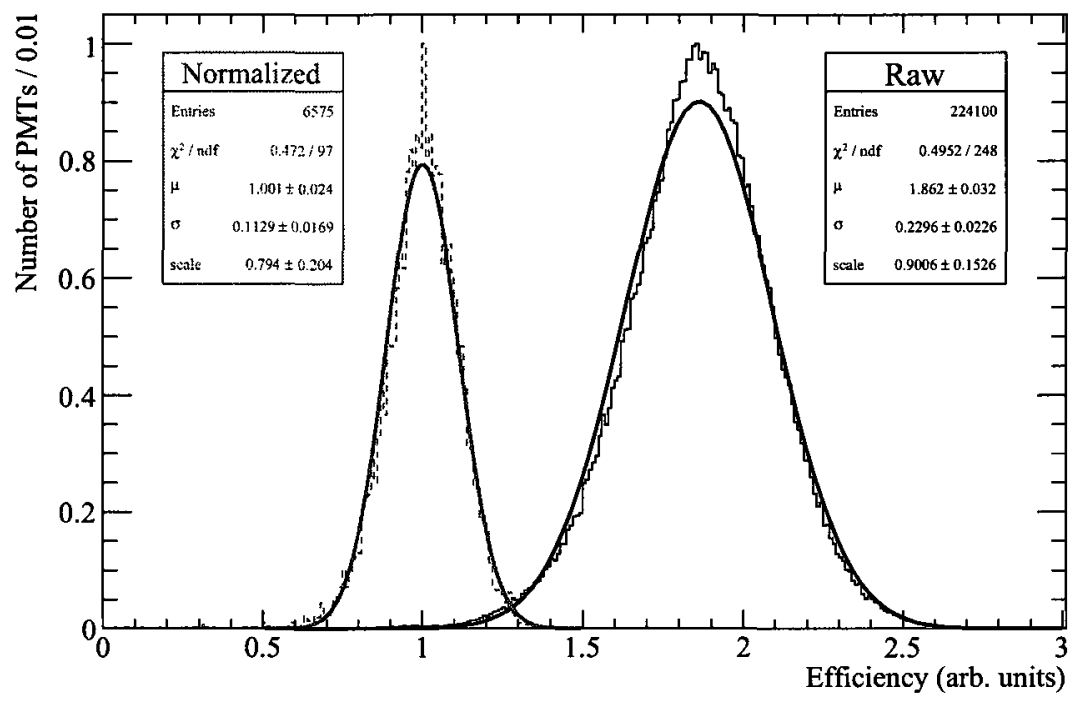

Figure 3.1: Raw and normalized efficiencies extracted from the October/03 data at 500 $\mathrm{nm}$. Both distributions are scaled by their maximum value.

tion 3.3.3 explains how to incorporate the efficiency spread information correctly into the Occupancy fit via the PMT variability term $v_{i j}$ in Equation (3.6).

Following the above procedure, the efficiencies from $\mathrm{D}_{2} \mathrm{O}$ and salt scans were extracted. Figure 3.2 shows the strong correlation and small relative differences of the relative efficiencies obtained from the $\mathrm{D}_{2} \mathrm{O}$ scans. Approximately $65 \%$ of the 9438 PMTs could be characterized; PMTs that could not be calibrated were given a default value $\epsilon_{j}=1.0$ and are not included in the figure. The comparison shows that the PMT relative efficiencies have not changed significantly over the course of the salt phase, corresponding to a three-year period. 


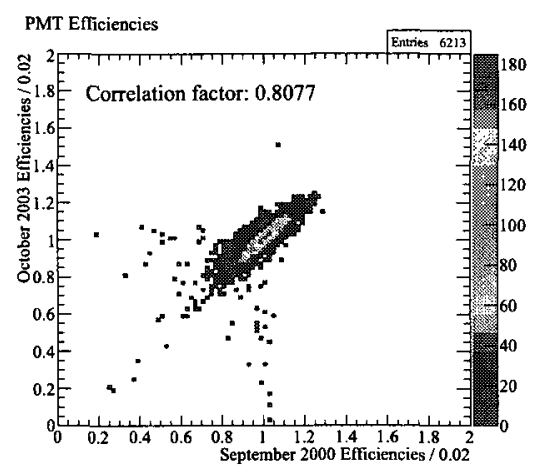

(a) $386 \mathrm{~nm}$.

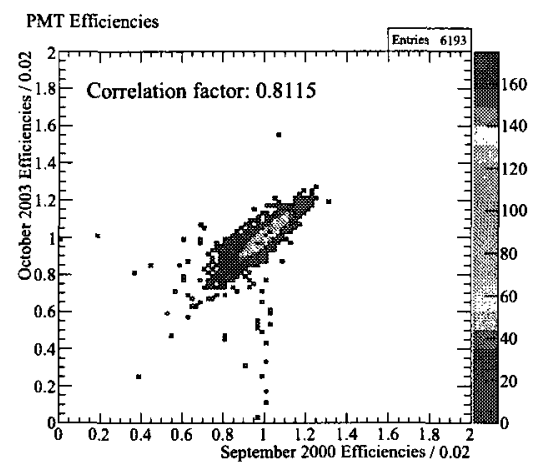

(c) $420 \mathrm{~nm}$.

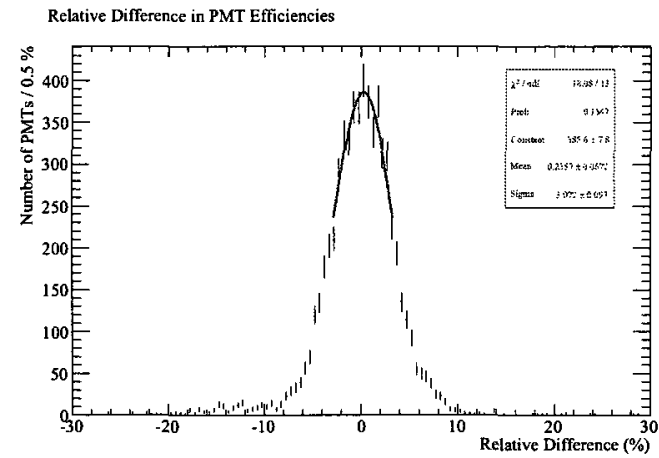

(b) $386 \mathrm{~nm}$.

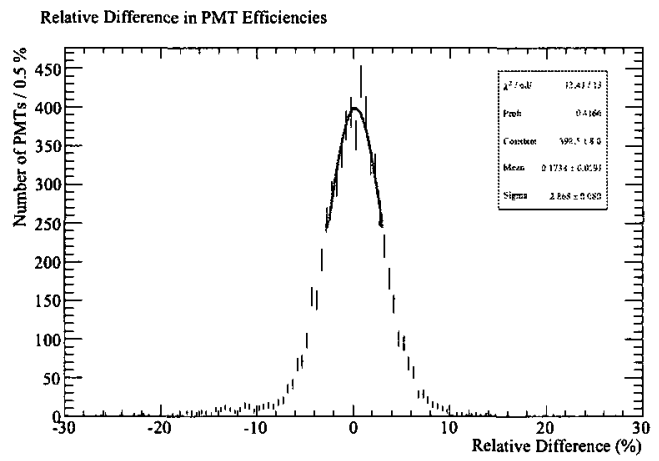

(d) $420 \mathrm{~nm}$.

Figure 3.2: (color) Correlation and relative difference, in percent, between the September/00 and October/03 PMT relative efficiencies at 386 and $420 \mathrm{~nm}$.

\subsubsection{Statistical Uncertainties}

Figure 3.3 shows the statistical uncertainties, $\Delta \epsilon_{j}$, associated with the extraction of the efficiencies from the $\mathrm{D}_{2} \mathrm{O}$ scans. Figure $3.3 \mathrm{a}$ shows that the distributions are peaked near $5.2 \%$ and $5.5 \%$ for the respective scans with small tails towards larger uncertainties. Figure 3.3b displays the same information as a function of the PMT $z$-coordinates to verify that there are no biases associated with the PMT spatial coordinates. The two profiles have the same basic features even if they are slightly different at low and high $z$ values. The mean statistical uncertainty on the extracted 


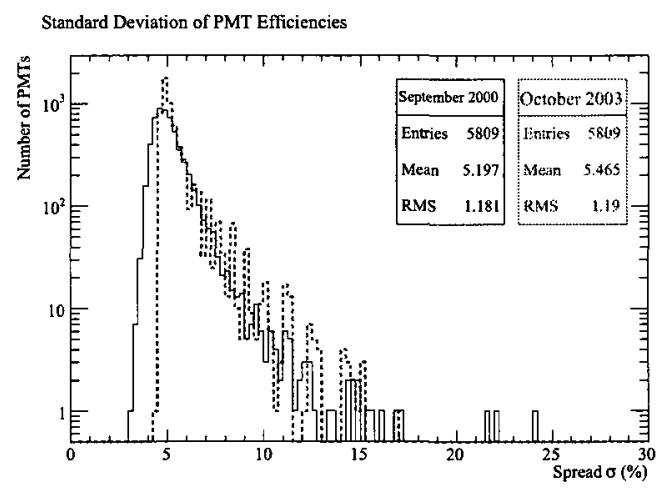

(a) Distribution of $\Delta \epsilon_{j}$.

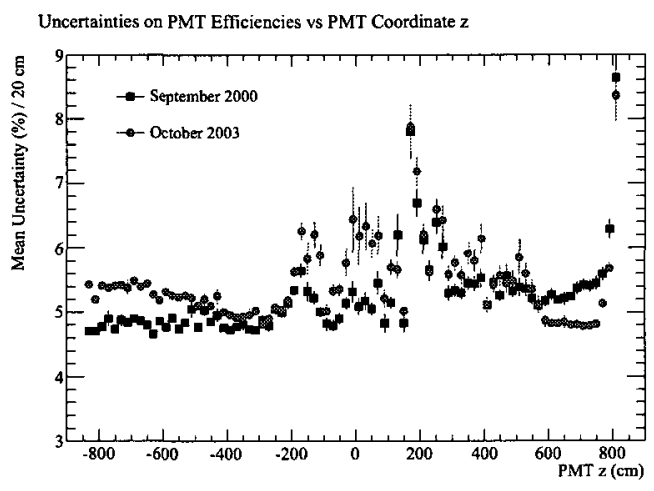

(b) $\Delta \epsilon_{j}$ vs $z$.

Figure 3.3: Distribution of the uncertainties on the PMT efficiencies for the September $/ 00$ and October $/ 03$ scans at $420 \mathrm{~nm}$. The distributions are made by requiring that the PMTs have a valid efficiency in both scans simultaneously for comparison purposes. PMT with less than five raw efficiencies were left out of the calculation.

relative efficiencies is of the order of $5 \%$.

\subsubsection{PMT Variability}

The PMT variability is the measure of the PMT-to-PMT variations of the efficiencies. Although its nature is mainly statistical, the variability is treated as a systematic uncertainty on the relative efficiency measurement that only applies to OCAs through the term $v_{i j}^{2}$ in Equations (3.4) and (3.6): $v_{i j} \equiv O_{i j} \times v_{i j}\left(\theta_{\mathrm{PMT}}\right)$. The variability is the efficiency spread remaining after subtracting the counting statistics uncertainty on the occupancy as a function of the PMT incident angle $\theta_{\mathrm{PMT}}$ :

$$
\begin{aligned}
v_{i j}^{2}\left(\theta_{\mathrm{PMT}}\right) & =\sigma_{\text {total }}^{2}\left(\theta_{\mathrm{PMT}}\right)-\sigma_{\mathrm{stat}}^{2}\left(\theta_{\mathrm{PMT}}\right) \\
\sigma_{\text {total }}\left(\theta_{\mathrm{PMT}}\right) & =\frac{\sigma_{\epsilon}}{\mu_{\epsilon}} \\
\sigma_{\text {stat }}\left(\theta_{\mathrm{PMT}}\right) & =\frac{1}{\sqrt{O_{i j}}} .
\end{aligned}
$$




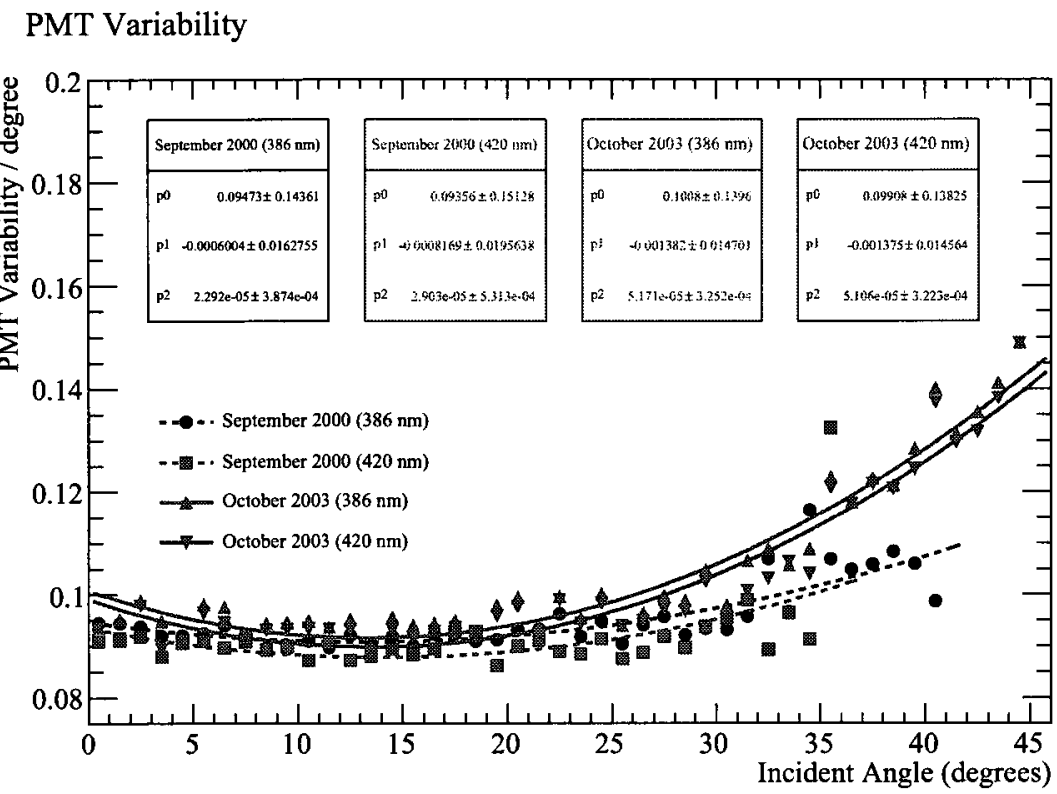

Figure 3.4: $\mathrm{PMT}$ variability as a function of PMT angle of incidence, $v_{i j}\left(\theta_{\mathrm{PMT}}\right)$, for the September/00 and October/03 scans at 386 and $420 \mathrm{~nm}$. A second order polynomial function is used to parameterize the binned functions.

Both $\sigma_{\text {total }}$ and $\sigma_{\text {stat }}$ are defined such that $v_{i j}\left(\theta_{\mathrm{PMT}}\right)$ is relative to $O_{i j}$. Here, $\sigma_{\epsilon}$ and $\mu_{\epsilon}$ are the RMS and mean values of the raw efficiency distribution in each $\theta_{\mathrm{PMT}}$ bin, therefore $\sigma_{\text {total }}$ also contains a contribution from the run normalizations mainly responsible for the differences depicted in Figure 3.1. The efficiency spread $\sigma_{\epsilon}$ generates the dependence on $\theta_{\mathrm{PMT}}$.

Figure 3.4 shows that the relative PMT variability data, $v_{i j}\left(\theta_{\mathrm{PMT}}\right)$, from the $\mathrm{D}_{2} \mathrm{O}$ scans are well described by second order polynomials as a function of $\theta_{\mathrm{PMT}}$. The results were similar for the salt phase [85]. The variability at high incident angles had increased in October/03 compared to September/00, possibly due to the degradation of the PMT reflectors or debris at the bottom of the AV. This additional systematic error is of the order of $10 \%$ and properly rescales the $\chi^{2}$ to take into account the spread of the overall raw efficiency distribution of Figure 3.1. 


\subsubsection{Local Response Variations}

The relative efficiencies of the $\mathrm{D}_{2} \mathrm{O}$ scans were extracted and plotted as a function of the PMT coordinates to verify if there were local variations in the detector. Figure 3.5 shows the efficiencies as a function of the PMT $x$ and $z$ coordinates. The efficiencies have similar profiles for both wavelengths. The $x$-coordinate profile has only a few outliers up to $4 \%$, showing stability across the $x$-axis. Similar stability prevails for the $y$-axis. However the $z$-profile shows an asymmetry for both wavelengths in both scans, leading to a $6 \%$ drop of the efficiencies in the upper hemisphere of the detector (see $[84,86]$ for the comparison of all wavelengths and for equivalent figures for salt scans). A residual Earth magnetic field could explain an up-down difference in the efficiencies. This could happen if the magnetic fields around the SNO detector were not totally canceled; compensating coils were installed in the SNO cavern for this purpose [21]. Otherwise there are yet no explanations for this asymmetry.

Similarly, early analyses of the $\mathrm{D}_{2} \mathrm{O}$ and salt data with a low energy threshold have shown that the energy systematic uncertainties depended on the detector coordinates [83]. The inclusion of the PMT efficiencies and the observed $z$-profile potentially fixed this situation and thus reduced the spatial dependence of the energy systematic uncertainties.

\subsection{Extraction of the Optical Model Parameters}

The optical model parameters, also called optical constants, have already been extracted during the NCD commissioning phase with improvements to the PMT angular response [87]. In this section, the main goal is to compare the Occupancy-Ratio (Oc- 


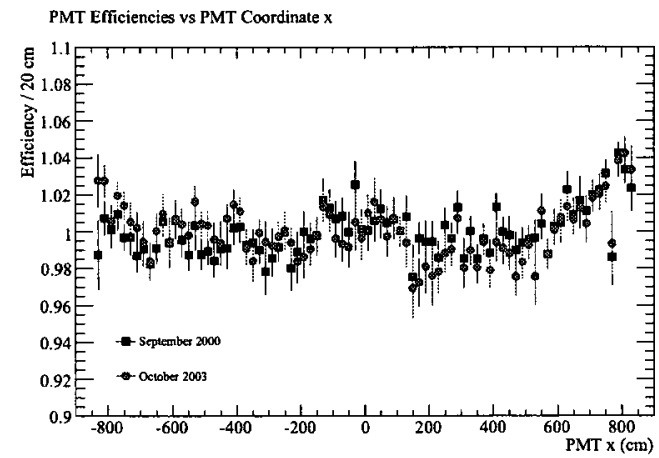

(a) $\epsilon_{j}$ vs $x$ at $386 \mathrm{~nm}$.

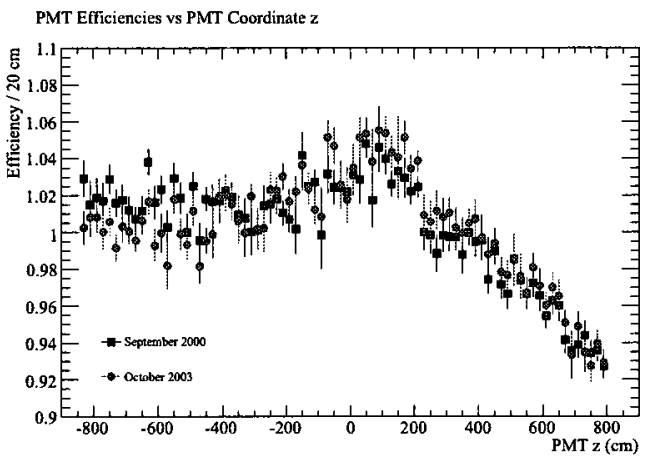

(c) $\epsilon_{j}$ vs $z$ at $386 \mathrm{~nm}$.

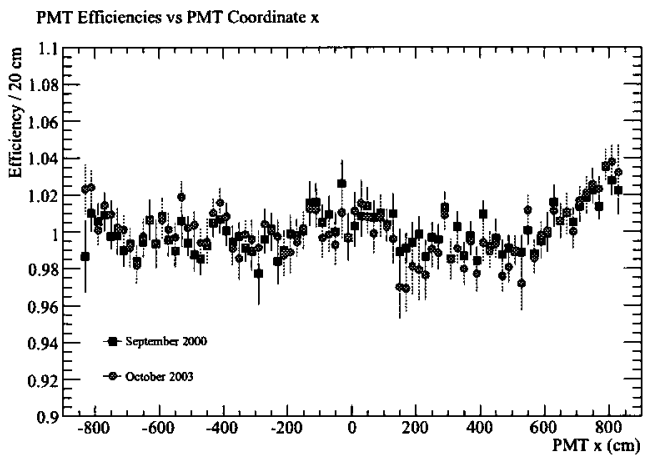

(b) $\epsilon_{j}$ vs $x$ at $420 \mathrm{~nm}$.

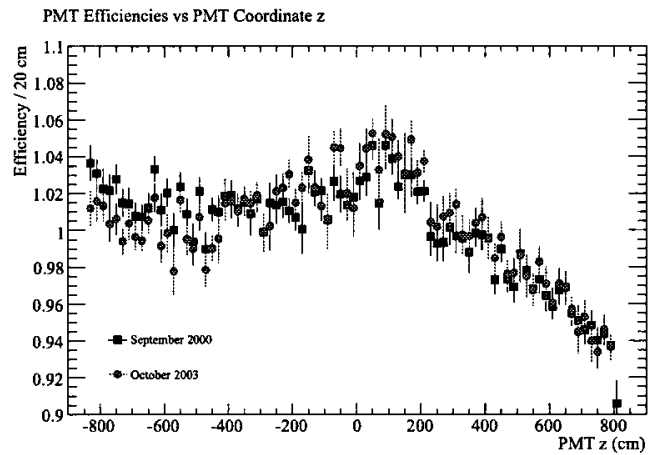

(d) $\epsilon_{j}$ vs $z$ at $420 \mathrm{~nm}$.

Figure 3.5: PMT relative efficiencies as a function of PMT coordinates in the detector at 386 and $420 \mathrm{~nm}$ extracted from the $\mathrm{D}_{2} \mathrm{O}$ scans. The points shown are mean values and the error bars indicate the uncertainty on the mean values in each $20-\mathrm{cm}$ bin.

cRatio) and Occupancy-Efficiency (Occupancy) extraction methods using the data of the $\mathrm{D}_{2} \mathrm{O}$ scans. This serves as a verification that the input efficiencies and variabilities can be trusted during the NCD phase.

The efficiencies and variability functions were extracted from the September/00 scan and used as inputs for both the September/00 and October/03 Occupancy fits. The former comparison tests the self-consistency of the method and the latter its application procedure. 


\subsubsection{Results and Comparison of the Methods}

The $\mathrm{D}_{2} \mathrm{O}$ and $\mathrm{H}_{2} \mathrm{O}$ attenuation coefficients as a function of wavelength and the PMT angular responses as a function of $\theta_{\mathrm{PMT}}$ are shown in Figures 3.6 and 3.7, respectively. In the case of the September/00 scan, the Occupancy fit uses its own set of efficiencies and variability to minimize the $\chi^{2}$ of Equation (3.6). The results from the OccRatio and Occupancy fits agree well within the statistical uncertainties. It is the case for all parameters shown.

In the analysis of the October/03 scan, the Occupancy method uses the efficiencies and variability functions from the September/00 scan. The results of the Occupancy method are compared to the OccRatio method, where the OccRatio results are shown with total uncertainties, which include a set of systematic uncertainties. The systematic uncertainties included in the October/03 error bars are a subset from the list of uncertainties explained in detail in Section A.2 of Appendix A. Their effect is larger on the media attenuations than on the PMT angular response. The conclusion is that the results agree within the expected total uncertainties, even after using the PMT efficiencies that were extracted three years before!

\subsubsection{Systematic Uncertainties due to PMT Efficiencies}

The effect of the uncertainties associated with the extraction of the PMT efficiencies described in Section 3.3 must be included in the systematic uncertainties of the optical constants. This subsection introduces two new systematic uncertainties, that in addition to the PMT variability, will be taken into account in Chapter 4. 


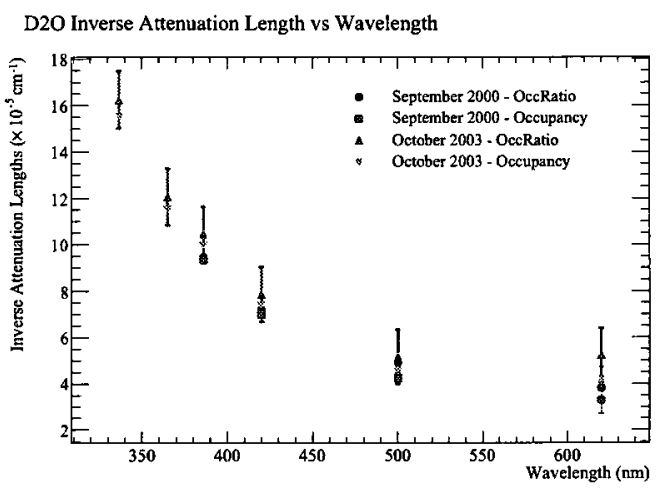

(a) $\mathrm{D}_{2} \mathrm{O}$ attenuations.

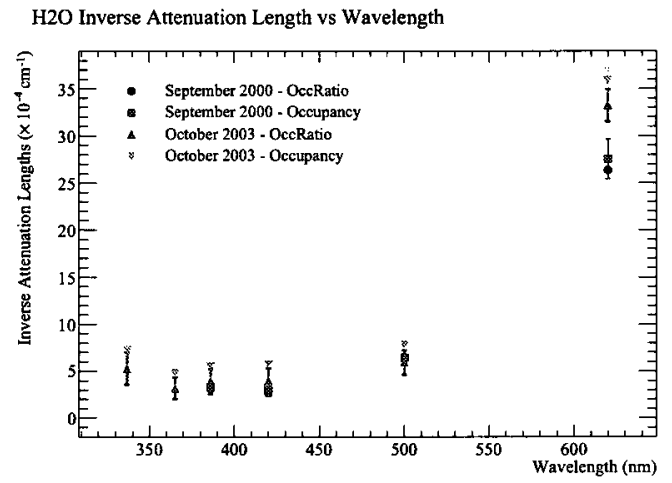

(b) $\mathrm{H}_{2} \mathrm{O}$ attenuations.

Figure 3.6: Media attenuation coefficients obtained from the September $/ 00$ and October $/ 03$ scans. Results for both the Occupancy-Ratio (OccRatio) and and Occupancy-Efficiency (Occupancy) fits are shown. The total error bars are shown for the October/03 results obtained with the OccRatio method, otherwise errors are statistical only.

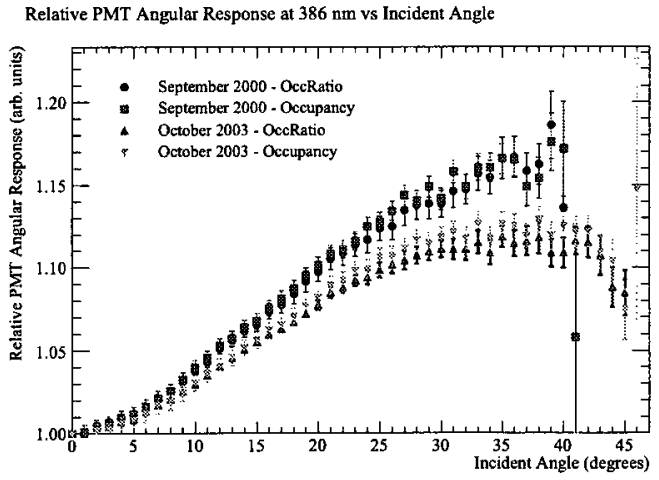

(a) $386 \mathrm{~nm}$.

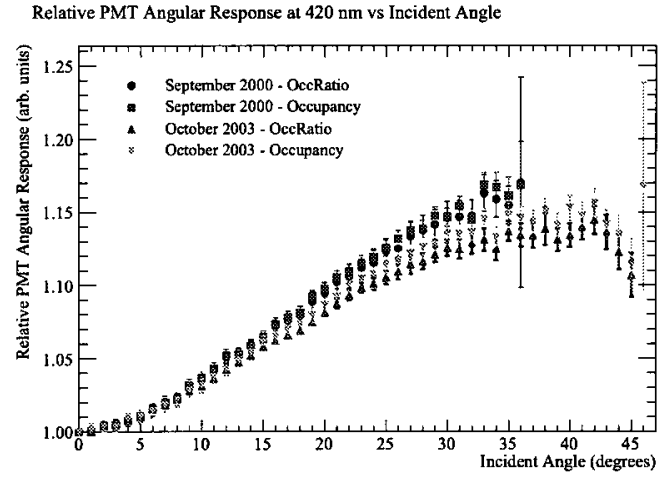

(b) $420 \mathrm{~nm}$.

Figure 3.7: PMT relative angular response obtained from the September/00 and October $/ 03$ scans at 386 and $420 \mathrm{~nm}$. Results for both the Occupancy-Ratio (OccRatio) and and Occupancy-Efficiency (Occupancy) fits are shown. The total error bars are shown for the October/03 results obtained with the OccRatio method, otherwise errors are statistical only. 


\section{Extraction Uncertainties}

In Subsection 3.3.2, the mean statistical uncertainty $\Delta \epsilon_{j}$ was found to be around $5 \%$. To take into account the effect of this average spread on the optical parameters, the efficiencies were randomly shifted using a random normal distribution $\mathrm{N}(0,0.05)$ which prevents the mean efficiency of 1.0 from being shifted by a large amount. The fit was repeated with the shifted efficiencies and differences in the optical parameters were observed. The $\mathrm{D}_{2} \mathrm{O}$ and $\mathrm{H}_{2} \mathrm{O}$ attenuations changed by $10 \%$ at all wavelengths, and the PMTR bins systematically shifted down by about $0.5 \%$.

\section{Detector Top-Down Asymmetry}

In Subsection 3.3.4, the $z$-profile of the efficiencies clearly showed an asymmetry in the detector. The effect of the top-down asymmetry was investigated by repeating the Occupancy fit using two independent PMT sets, one at the bottom $(z<0 \mathrm{~cm})$ and the other at the top $(z>0 \mathrm{~cm})$ and fitting for two PMTR functions. The concentrators in the lower half of the PSUP are of lower quality vis-a-vis resistance to aging than those in the upper half [90], but there is no evidence that this is what caused the observed asymmetry. Although statistical fluctuations were observed, the PMTR for the top part of the detector was found to be $2-3 \%$ lower at large incident angles. A conservative estimate of this effect on the averaged PMTR was set to $\left(R^{\text {top }}-R^{\text {bot }}\right) / R^{\text {ave }} \simeq 1 \%$.

\section{Extrapolation to the NCD Phase}

The September/00 efficiencies did not pose a problem for the analysis of the October/03 OCA data. However the electronics backplane replacement in the NCD 
commissioning phase could change the patterns in the efficiencies for the NCD phase. The potential changes in the efficiencies could be taken into account using the simulated charge spectrum in SNOMAN to predict the efficiencies due to threshold and gain alone $[91,92]$. The PMT efficiencies measured before the backplane replacement could then be corrected to take into account the high-voltage changes and extrapolated to the NCD phase with confidence without additional systematic uncertainties.

\subsubsection{Summary}

The relative PMT efficiencies were extracted from OCA data to improve various SNO analyses. In neutrino data analyses, the use of the extracted efficiencies in the data processing and $\mathrm{MC}$ simulations results in a more accurate response model of the detector and reduces the spatial dependence of the energy estimators.

With respect to OCA analyses, the efficiencies measured in the NCD commissioning phase served as a benchmark measurement that was used throughout the NCD phase as input to the Occupancy fit method. The comparison of the optical constants using two $\mathrm{D}_{2} \mathrm{O}$-phase scans demonstrated the technique could be used. The Occupancy method has no major advantages for $\mathrm{D}_{2} \mathrm{O}$ - and salt-phase scans, but provides a way to increase the fit statistics lost to shadowing effects for NCD-phase scans.

Systematic uncertainties associated with the utilization of the PMT efficiencies in the Occupancy method were identified and evaluated from the $\mathrm{D}_{2} \mathrm{O}$ scans. The latter were added to a group of new systematic uncertainties evaluated in the NCD phase. Chapter 4 follows with the OCA of the SNO detector in the NCD phase. 


\section{CHAPTER 4}

\section{Optical Calibration in the NCD Phase}

This chapter describes the optical calibration (OCA) techniques developed to account for the different configuration of the SNO detector in the Neutral Current Detector (NCD) phase. The optical model parameters introduced in Chapter 3 are extracted from the laserball calibration data collected throughout the NCD phase using the PMT efficiencies measured during the NCD commissioning phase as a baseline. The systematic uncertainties associated with the new OCA are defined and their effect on the SNO position and energy estimators for the analysis of the NCD-phase neutrino data are investigated.

\subsection{Introduction}

This introductory section describes the changes brought to the laserball (LB) source deployment plan, lists the data sets selected for the extraction of the NCD-phase optical constants, and outlines the steps of the OCA analysis.

\subsubsection{Optical Calibration Method and Data Sets}

A standard OCA scan consists of many runs at six different wavelengths, preceded by high statistics PCA runs at $500 \mathrm{~nm}$. This scheme was modified in the following ways 
to adapt to the NCD array geometry and improve the quality of the calibration.

First, a set of LB positions outside the NCD array (c.f. Figure 2.2) were added to the scans to allow a full-half access of the detector solid angle without NCD shadowing effects [93]. These runs are called horizontal runs because they were in the $z_{\mathrm{LB}}=0$ plane. Second, the positions inside the NCD array were optimized to reduce the number of shadowed PMTs [88]. Finally, the duration of strategic runs such as central and horizontal runs was doubled compared to previous phases. The above changes are referred to as the base changes to the OCA scan plan.

Table 4.1 lists the principal scans that were performed during the NCD phase. A more extensive list of scans is available in [94]. In addition to the base changes, as indicated in Table 4.1, selected scans were adjusted for various purposes. For instance, the February/06 scan was extended and performed with a finer positioning grid to improve the measurement of the NCD positions (see Section 4.3).

The new data selection rules reduce the statistics significantly in the NCD phase, as will be explained in Section 4.5. Overall, the typical number of data points (good PMTs, left after all cuts are applied) per wavelength is about $65 \%$ smaller than in

previous phases. However, the quality and quantity of the optical data collected in the NCD phase improved due to the above OCA modifications. Despite the new geometry and acceptance, the level of statistics per run in the raw data is approximately the same as in previous phases.

\subsubsection{Overview of the Analysis}

The rest of this chapter describes the analysis steps towards the extraction of the NCD-phase optical constants: starting with the base steps that were defined in previ- 


\begin{tabular}{|l|c|l|l|}
\hline \hline Scan & Julian Date & Characteristics & Goal \\
\hline \hline October 2003 & 10515 & Last non-NCD scan & $\begin{array}{l}\text { Reference for optical constants } \\
\text { Discover new features }\end{array}$ \\
\hline October 2004 & 10783 & First NCD scan & Almost uniform sampling \\
February 2005 & 11014 & Optimized positions & Minimize shadowing effects \\
May 2005 & 11092 & Regular scan & Stability check \\
October 2005 & 11250 & Light water scan & Light water attenuation \\
February 2006 & 11372 & Horizontal scan & Improve NCD position fits \\
June 2006 & 11486 & Trigger efficiency & Measure trigger efficiency \\
August 2006 & 11562 & Last optics scan & Stability check \\
\hline \hline
\end{tabular}

Table 4.1: Summary of the full optical scans taken during the NCD phase. The SNO Julian date, defined as the number of days which have passed since January $1^{\text {st }} 1975$, indicates the last day of a scan. The characteristics and goal columns refer to special features of a scan.

ous phases and leading to the new steps proper to the NCD phase. The basic design of the analysis kept the format in which previous calibrations were performed. Optical effects that alter the light intensity measured by the PMTs were either removed or accounted for to ensure an unbiased measurement of the optical properties of the detector. Specifically, new effects such as NCD partial shadowing and reflections were removed and corrected for, respectively.

Section 4.2 explains how the LB positions are obtained from the calibrated PMT hit times. Section 4.3 introduces the NCD position extraction method that uses the spatial shadowing patterns between the LB and PMT positions. Section 4.4 describes how the knowledge of the LB and NCD positions are combined to remove partially shadowed PMTs from the clean data set, and correct for the weak effect of NCD reflections. Finally, Section 4.5 gives the results of the optical constants and Section 4.6 describes the estimation of the effect of the systematic uncertainties on the SNO event vertex and energy fitters. 


\subsection{Laserball Source Positions}

There are many physical quantities in the optical model of Equation (3.1) that depend directly on the accurate determination of the LB positions. In the NCD phase, the LB positions are, in addition, important to determine the shadowing patterns due to NCDs at the PMTs. Therefore the accuracy with which the LB positions are determined is critical to OCAs. However, the method established for the $\mathrm{D}_{2} \mathrm{O}$ and salt phases was not upgraded significantly for the NCD phase. The main goal was to ensure the PMT charges and times behaved in the same way despite the potential effects of shadows and reflections. Hence this subsection explains briefly how the LB positions are obtained and gives an estimation of the total position uncertainty. More details can be found in $[56,81,95]$.

\subsubsection{PMT Times}

The PCA [96] determined the offsets and central times $t_{0}$, the discriminator walk corrections, and flagged bad time spectra associated with some PMT channels. For each PMT $j$, the counts as a function of time formed a distribution centered on the

mean time $\mu_{t}^{j}$ with a width $\sigma_{t}^{j}$. The mean and width were found from a timing histogram, built from 320.25 -ns-wide bins, by maximizing the counts in a sliding \pm 4 ns window. The mean $\mu_{t}^{j}$ was used as the time variable for PMT $j$; the error on the mean $\sigma_{\mu_{t}}^{j}=\sigma_{t}^{j} / \sqrt{32}$ was used as the error. 


\subsubsection{Source Position Fit}

The LB position $i$ was found by minimizing the $\chi^{2}$ sum of all time residuals:

$$
\chi^{2}=\sum_{j}^{N_{\mathrm{PMT}}^{i}} \frac{\left(\mu_{t}^{j}-t_{\text {flight }}^{j}\right)^{2}}{\left(\sigma_{\mu_{t}}^{j}\right)^{2}},
$$

where $t_{\text {flight }}^{j}$ is the expected time-of-flight for $\operatorname{PMT} j$, and $N_{\mathrm{PMT}}^{i}$ is the number of PMTs with valid times in run $i$. To improve the accuracy, $t_{\text {fight }}^{j}$ was calculated with a full path calculation through the $\mathrm{D}_{2} \mathrm{O} / \mathrm{AV} / \mathrm{H}_{2} \mathrm{O}$ boundaries assuming the $\mathrm{AV}$ and PSUP coordinate systems were concentric. The recorded manipulator position served as the initial guess for the fit which made the algorithm converge quickly.

Within a given run, the $N_{\mathrm{PMT}}^{i}$ values of $\mu_{t}^{j}$ and $\sigma_{t}^{j}$ were used for quality control. The time width $\left(\sigma_{t}\right)$ mean value and spread, defined respectively as $\mu_{\sigma_{t}}$ and $\sigma_{\text {run }}$, were calculated and PMTs with $\sigma_{t}^{j}>3 \sigma_{\text {run }}$ were removed. Depending on the position, this cut removed up to $5 \%$ of the PMTs in a run. Other cuts removed PMTs with extremely low or high occupancies. MC studies revealed that the above new checks on PMT times and occupancies, although improving the confidence in the position fits, have not made significant differences in the fitter performance [97].

\subsubsection{Uncertainties}

The average statistical fit uncertainty on the optical center of the LB was below $1 \mathrm{~cm}$. An additional systematic uncertainty of $2 \mathrm{~cm}$ was assigned based on the observed differences between the manipulator and fitted positions. Efforts to understand these differences with PCA corrections [98] and PCA-less fits [95] did not reduce that 
systematic uncertainty. Therefore the average total uncertainty assigned to the LB position was estimated to be $\Delta r_{\mathrm{LB}}=2 \mathrm{~cm}$. This is an important uncertainty at the base of the evaluation of many other systematic uncertainties.

\subsection{Extraction of the NCD Positions}

Section 2.1.3 described how the NCDs were attached at the bottom of the AV, floating in $\mathrm{D}_{2} \mathrm{O}$, but stabilized at the top by the electronic cables. In this section, the NCD positions are extracted from the OCA data for two reasons. The main reason is for general calibration purposes: the NCD positions must be known. This is especially important for OCAs in which shadowing patterns are identified using the NCD positions. The second reason is for monitoring. Calibrations that involve moving the source and ropes, or water circulation, could potentially displace the NCDs. Therefore it is desirable to extract the positions of the NCDs on a scan-by-scan basis and update the geometry whenever a shift is observed.

There are two methods to measure the NCD coordinates. One method used a laser system during the NCD commissioning phase to locate the top of the counters [99]. The precision of that method verified that the NCDs were close to their target positions. The other method, the optics method, used the shadowing patterns in the PMT data, and is explained in this section. Although more precise on average, this method relies strongly on the precision of the measured LB coordinates, which propagates directly into the NCD position uncertainties. Despite the dependence on the LB positions, the optics method is the primary approach employed to determine the NCD positions since it reduces the uncertainties by averaging them with multiple measurements. More details on the optics method can be found in [97, 100]. 


\subsubsection{Method of Relative Occupancy Mapping}

In a given run, the number of detected photons depends on the factors in Equation (3.1). In the NCD phase, the NCD array stood between the LB and many PMTs and introduced additional effects that were not modeled. The goal of mapping the relative occupancy is to isolate the latter effects as a function of the detector coordinates to find the NCD positions, assuming the NCDs were the only cause of the observed effect.

\section{Geometry of the Low Occupancy Region}

The low occupancy region is a surface containing the average relative PMT occupancies, projected in the $x y$-plane, concentrated around the expected position of a NCD. The surface is a 2D histogram with limits corresponding to the detector $x$ and $y$ coordinates and adjustable bin sizes. The limits were set to $\pm 15 \mathrm{~cm}$ around a given default NCD position.

The bin size was selected to describe the resolution in the detector $(x, y)$ coordinates accurately. The strategy adopted was to choose the bin size to correspond to the resolution inhibited by the uncertainty on the LB positions, the main systematic uncertainty, so that the latter was propagated in the method automatically.

Let $\delta r$ be the hypotenuse size of the bins. The average value of $\delta r$ was evaluated using a set of MC-generated LB positions and by calculating, with the geometry code, the various distances between the relevant objects (LB, NCD, PMT) for each $\mathrm{LB}$ position. The average distances $d(\mathrm{LB} \rightarrow \mathrm{NCD})$ and $d(\mathrm{NCD} \rightarrow \mathrm{PMT})$ were found to be $360 \mathrm{~cm}$ and $880 \mathrm{~cm}$, respectively. To simulate the LB-position uncertainty of $2 \mathrm{~cm}$, the LB positions were shifted, while the NCD and PMT positions were kept 
fixed, such that the lines open with angle $\tan \theta=2 \mathrm{~cm} / d(\mathrm{LB} \rightarrow \mathrm{PMT})$. The distance of interest is the shift at the NCD position, which was evaluated using $\tan \theta$ and the average NCD-PMT distance:

$$
\delta r=\tan \theta \times d(\mathrm{NCD} \rightarrow \mathrm{PMT})=\frac{2 \mathrm{~cm}}{(880+360) \mathrm{cm}} \times 880 \mathrm{~cm} \simeq 1.5 \mathrm{~cm} .
$$

Thus, the square bin diagonal length can be as long as $1.5 \mathrm{~cm}$ corresponding approximately to a length of $1 \mathrm{~cm}$ on each side of the bin. Therefore, in the following, it was assumed that setting the bin side length to $1 \mathrm{~cm}$ takes into account the average $2 \mathrm{~cm}$ uncertainty on the LB positions.

Figure 4.1 shows how the relative occupancies are filled into the region where a NCD is likely to be found. For each selected PMT in a given run, the LB and PMT positions were used to calculate the optical paths through the $\mathrm{D}_{2} \mathrm{O}, \mathrm{AV}$, and $\mathrm{H}_{2} \mathrm{O}$. The direction of the vector in the $\mathrm{D}_{2} \mathrm{O}$, or the photon trace, was projected into the low occupancy surface such that each bin of the surface along the vector was weighted by the relative occupancy of the corresponding PMT. Figure 4.1b shows how the low occupancy region shapes from the input of many LB positions.

\section{Selection of Photon Traces}

The shadowed PMTs were selected with restrictions. The base cut removed unwanted optical effects described in Chapter 3 and multiple-shadowing, leaving only 'clear' NCD shadows in the PMT data. The selected PMTs were kept to calculate the run average occupancy, which is the mean number of hits per PMT in a run, to normalize the PMT counts such that all runs could be compared in the same units. After normalization, average PMTs had occupancies close to one, and the PMTs that were 


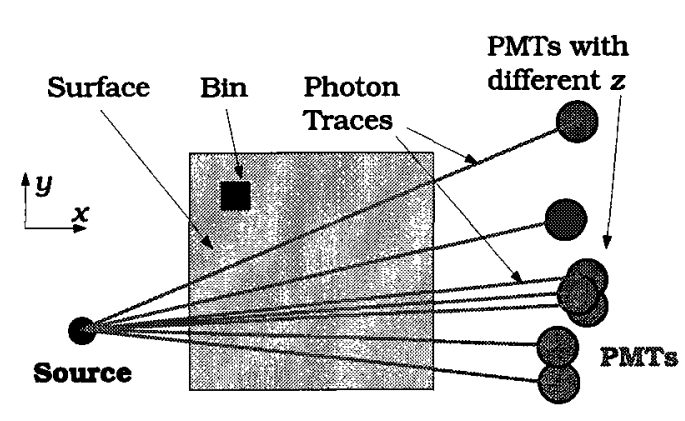

(a) Single run.

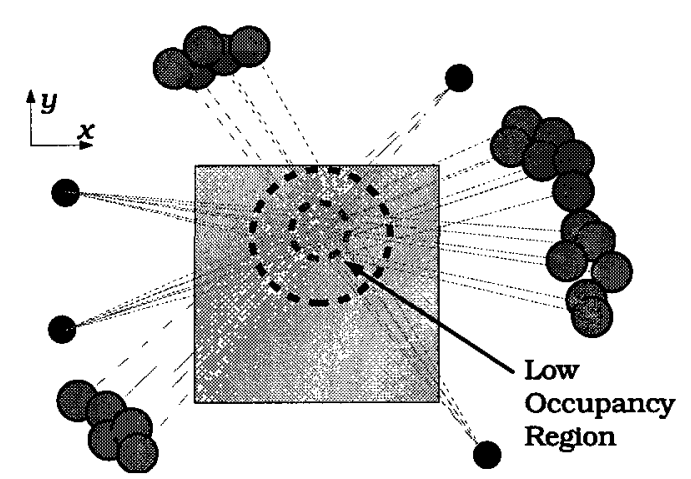

(b) Many runs.

Figure 4.1: (color) Mechanics of the relative occupancy mapping method. The smaller red points represent the LB source while the orange circles depict the PMTs. Relative occupancies weight the photon traces between the source and the PMTs. Repeating the process with many runs reveals a region of low occupancy within the predefined surface. The refraction at the $\mathrm{D}_{2} \mathrm{O}-\mathrm{AV}-\mathrm{H}_{2} \mathrm{O}$ interfaces was taken into account but not shown for clarity.

shadowed had occupancies that were significantly lower. Normalized occupancies in the range [0.01-20] from runs with at least 20 PMTs were selected to construct the low occupancy region.

\section{Minimization Function}

Each bin of the surface contained the average of all normalized occupancies. The surface was inverted and then scanned to find a peak of low occupancy. The peak was well-described by a $2 \mathrm{D}$ normal distribution [101]:

$$
f(x, y)=\frac{N}{2 \pi \sigma_{x} \sigma_{y}} \exp \left\{-\left(\frac{x-\mu_{x}}{\sigma_{x}}\right)^{2}\right\} \exp \left\{-\left(\frac{y-\mu_{y}}{\sigma_{y}}\right)^{2}\right\}+C
$$

where $N$ is a normalization factor, $\mu_{i}$ the NCD coordinates, $\sigma_{i}$ the standard deviation for each coordinate, and $C$ an offset. The offset $C$ was initialized as the inverse of the 
mean occupancy, usually a number close to one, and ensured that the fitter found a peak of low occupancy relative to that baseline. The fit was achieved by TMinuit [102] to find the six parameters that minimized the difference between $f(x, y)$ and the inverse occupancy map. The six parameters were extracted simultaneously, but only the NCD coordinates $\left(\mu_{x}, \mu_{y}\right)$, with their uncertainties were kept for further analysis.

\subsubsection{Results}

As indicated in Table 4.1, the February/06 scan was optimized for the extraction of the NCD positions. The results from that scan and the average from all scans are given below.

\section{Central Positions}

The central position corresponds to the case where the projected surface was built from all $z$ values with the surface itself being at $z=0$. Figure 4.2 shows the low occupancy regions in the $x y$-plane for NCDs M4 and M1, with the coordinate results

and confidence levels (CL) extracted from the February/06 data. The NCD-Average positions, given in Table 4.2, correspond to the NCD-phase average fit and the size is the physical radius of the NCDs $(2.579 \mathrm{~cm})$. For these particular counters, the 99\% CL contours obtained from the February/06 scan cover the physical size of the NCDs. The uncertainties were much smaller after all scans were used to calculate the weighted average, as shown in Table 4.2. A typical coordinate uncertainty on $x$ and $y$ was $0.5 \mathrm{~cm}$.

Some NCD coordinates and uncertainties could be affected by the integration in $z$ because of their length of approximately $10 \mathrm{~m}$. The fit was repeated with $k$ subsets of 


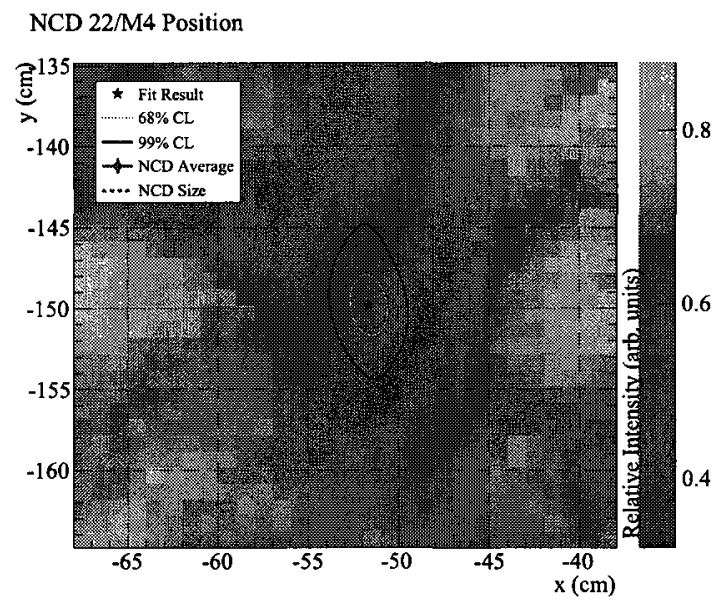

(a) NCD 22/M4.

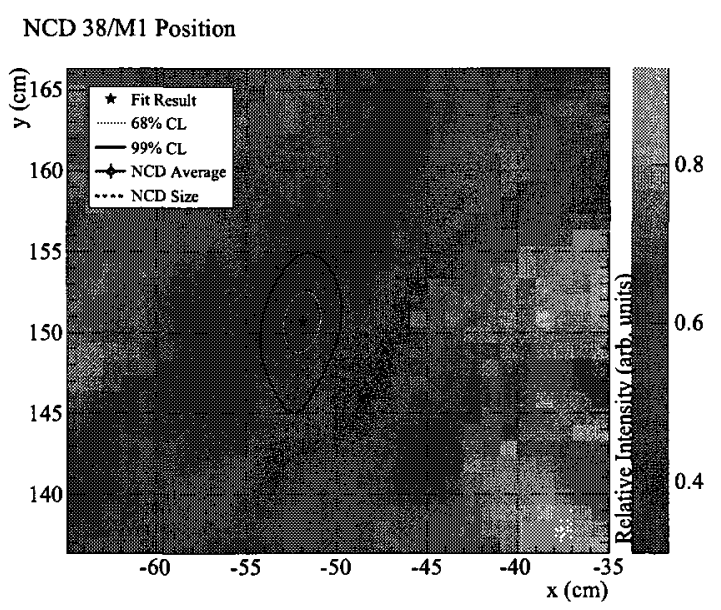

(b) NCD 38/M1.

Figure 4.2: Selected M-series NCD positions fitted from the February/06 scan data. The relative intensity data are averaged over all $z$-values. The confidence level (CL) contours are representative of the total position uncertainty in the $x y$-plane at $z=0 \mathrm{~cm}$. The average positions are taken from Table 4.2 .

integrated $z$-ranges to verify if the sub-coordinates, $\mu_{x}\left(z_{k}\right)$ and $\mu_{y}\left(z_{k}\right)$, were consistent with the $z$-integrated values. The angle of the NCDs with respect to the $z$-axis of the detector, called tilt, was extracted from the relative alignment of the sub-positions.

\section{Tilts}

The deviations $\theta_{x}$ and $\theta_{y}$ were extracted by dividing each NCD in three sub-sections [100]. To optimize the statistics in each sub-section their range in $z$ was set asymmetrically: the bottom section went from the anchor, $z_{\min }$, to $z=-100 \mathrm{~cm}$, the central section from $z=-100 \mathrm{~cm}$ to $z=+100 \mathrm{~cm}$, and the top section from $z=+100 \mathrm{~cm}$ to the readout cable, $z_{\max }$.

The coordinates $\mu_{x}\left(z_{k}\right)$ and $\mu_{y}\left(z_{k}\right)$ were obtained independently and then plotted against $z_{k}$. Figure 4.3 shows the results for NCDs M1 and M4. The uncertainties were extracted from the $99 \% \mathrm{CL}$ contours to account for the possible correlations and 


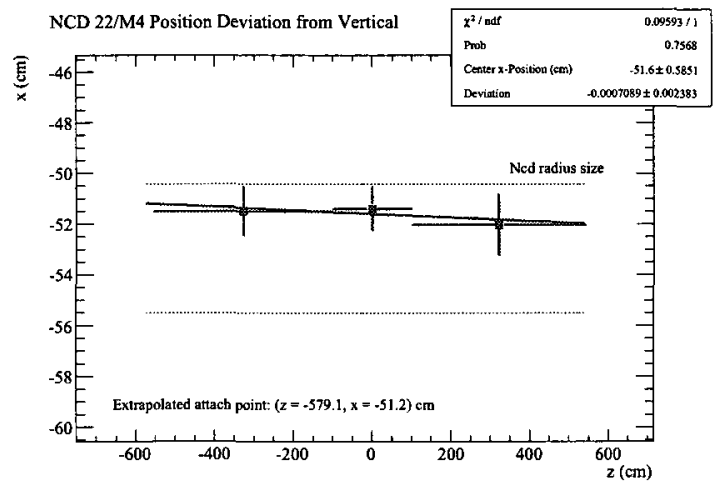

(a) NCD 22/M4 $\mu_{x}(z)$.

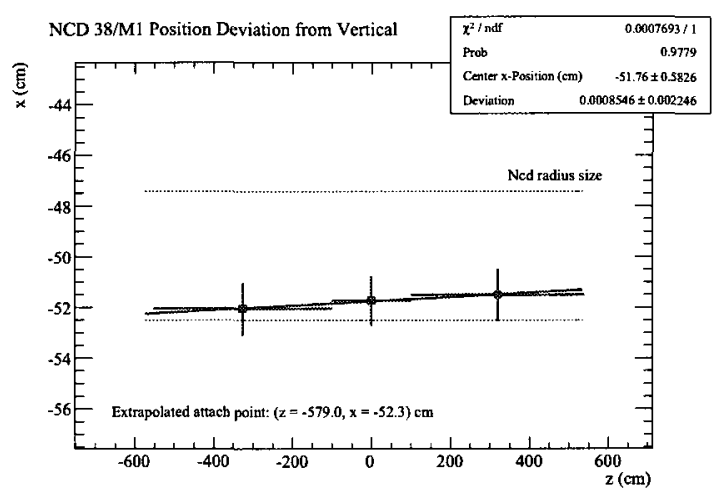

(c) NCD 38/M1 $\mu_{x}(z)$.

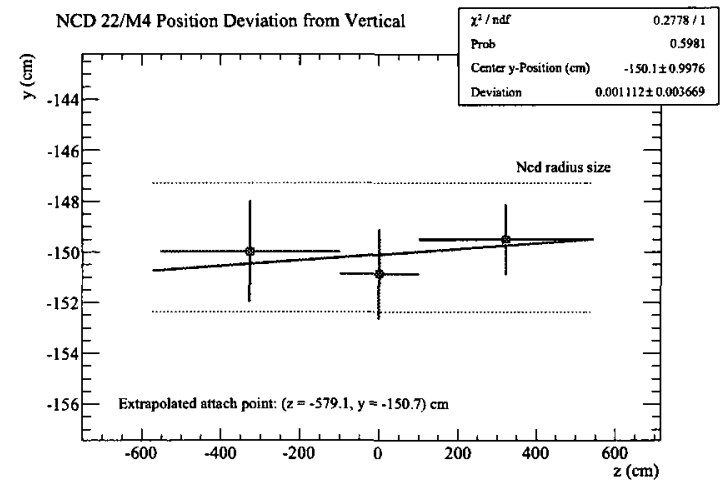

(b) NCD 22/M4 $\mu_{y}(z)$.

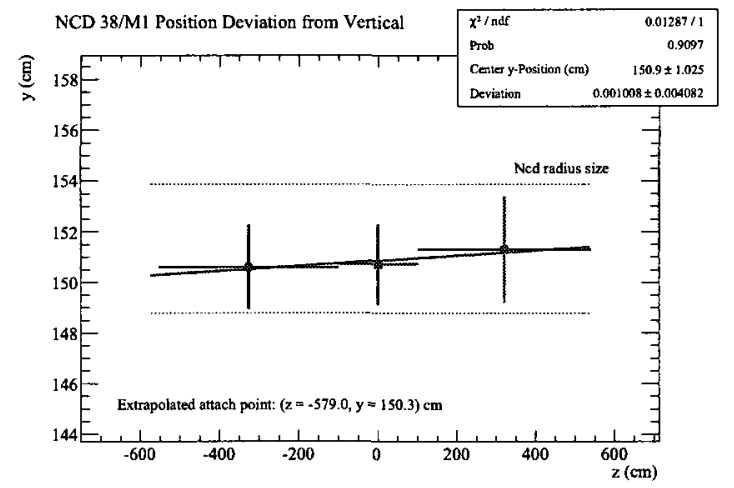

(d) NCD 38/M1 $\mu_{y}(z)$.

Figure 4.3: Selected M-series NCD tilts fitted from the February/06 scan. The uncertainties in the coordinates are extracted from the $99 \%$ CL contours. The NCD radius size is centered on the average positions from Table 4.2 .

NCD size effects. A straight line fit was performed to obtain the slope (tilt) and the position at $z=0 \mathrm{~cm}$. The anchor points of each NCD were obtained by extrapolating the line $z=z_{\min }$. The slope parameters are given in radians. Translating them into tilts in degrees gave values that were smaller than one degree. There were no NCD tilts larger than one degree found using this method. In addition the uncertainties were larger than one degree, hence no tilts were observed. Table 4.2 also contains the tilts $\theta_{x}$ and $\theta_{y}$ extracted from the February/06 scan. 


\begin{tabular}{|cc|rr|rc|}
\hline \hline $\mathrm{NCD}$ & \multicolumn{1}{|c|}{$\mu_{x}^{\text {avg }}$} & $\mu_{y}^{\text {avg }}$ & $\theta_{x}$ & $\theta_{y}$ \\
\hline \hline 00 & $\mathrm{~N} 4$ & $+46.63 \pm 0.38$ & $+47.53 \pm 0.29$ & -0.24 & -0.16 \\
01 & $\mathrm{M} 8$ & $+49.09 \pm 0.27$ & $+149.46 \pm 0.49$ & -0.06 & +0.03 \\
02 & $\mathrm{~K} 8$ & $+47.92 \pm 0.37$ & $+246.41 \pm 0.82$ & +0.16 & -0.55 \\
03 & $\mathrm{I} 7$ & $+48.92 \pm 0.49$ & $+338.27 \pm 0.43$ & +0.11 & -0.28 \\
04 & $\mathrm{~J} 8$ & $+149.32 \pm 0.50$ & $+247.72 \pm 0.61$ & -0.14 & +0.12 \\
05 & $\mathrm{~L} 2$ & $+147.84 \pm 0.41$ & $+147.09 \pm 0.45$ & -0.16 & -0.23 \\
06 & $\mathrm{~J} 7$ & $+247.25 \pm 0.53$ & $+146.76 \pm 0.50$ & -0.11 & -0.26 \\
07 & $\mathrm{M} 7$ & $+149.13 \pm 0.61$ & $+47.78 \pm 0.22$ & +0.07 & -0.05 \\
08 & $\mathrm{~K} 7$ & $+244.30 \pm 1.13$ & $+47.64 \pm 0.23$ & -0.35 & -0.04 \\
09 & $\mathrm{I} 8$ & $+337.50 \pm 0.88$ & $+44.40 \pm 0.33$ & -0.01 & -0.06 \\
\hline 10 & $\mathrm{I} 6$ & $+335.41 \pm 0.71$ & $-52.32 \pm 0.35$ & -0.27 & -0.06 \\
11 & $\mathrm{~K} 6$ & $+246.62 \pm 0.72$ & $-51.87 \pm 0.35$ & -0.18 & -0.01 \\
12 & $\mathrm{M} 6$ & $+149.65 \pm 0.52$ & $-48.62 \pm 0.22$ & +0.04 & +0.01 \\
13 & $\mathrm{~J} 6$ & $+244.52 \pm 0.55$ & $-150.14 \pm 0.45$ & -0.26 & +0.09 \\
14 & $\mathrm{~N} 3$ & $+43.79 \pm 0.34$ & $-48.41 \pm 0.28$ & -0.16 & +0.14 \\
15 & $\mathrm{~L} 3$ & $+143.20 \pm 0.40$ & $-147.66 \pm 0.43$ & -0.25 & +0.15 \\
16 & $\mathrm{~J} 5$ & $+143.75 \pm 0.42$ & $-248.55 \pm 0.54$ & -0.16 & +0.15 \\
17 & $\mathrm{M} 5$ & $+45.89 \pm 0.27$ & $-150.54 \pm 0.57$ & +0.11 & -0.14 \\
18 & $\mathrm{~K} 5$ & $+45.08 \pm 0.22$ & $-249.00 \pm 0.87$ & -0.14 & -0.01 \\
19 & $\mathrm{I} 5$ & $+43.68 \pm 0.35$ & $-335.15 \pm 1.15$ & +0.16 & +0.37 \\
\hline 20 & $\mathrm{I} 3$ & $-53.70 \pm 0.37$ & $-334.52 \pm 0.91$ & -0.01 & +0.44 \\
21 & $\mathrm{~K} 4$ & $-53.00 \pm 0.33$ & $-245.24 \pm 1.02$ & +0.11 & +0.51 \\
22 & $\mathrm{M} 4$ & $-52.96 \pm 0.26$ & $-149.82 \pm 0.49$ & -0.04 & +0.06 \\
23 & $\mathrm{~J} 4$ & $-154.66 \pm 0.45$ & $-247.50 \pm 0.61$ & +0.20 & +0.05 \\
24 & $\mathrm{~L} 4$ & $-149.99 \pm 0.45$ & $-146.14 \pm 0.51$ & +0.38 & +0.27 \\
25 & $\mathrm{~N} 2$ & $-51.41 \pm 0.38$ & $-48.06 \pm 0.28$ & +0.26 & +0.09 \\
26 & $\mathrm{~J} 3$ & $-248.94 \pm 0.79$ & $-145.20 \pm 0.43$ & +0.43 & +0.24 \\
27 & $\mathrm{M} 3$ & $-153.95 \pm 0.57$ & $-50.02 \pm 0.22$ & -0.10 & -0.05 \\
28 & $\mathrm{~K} 3$ & $-250.86 \pm 0.78$ & $-46.79 \pm 0.25$ & +0.25 & +0.07 \\
29 & $\mathrm{I} 4$ & $-340.60 \pm 0.08$ & $-47.23 \pm 0.48$ & +0.67 & -0.14 \\
\hline 30 & $\mathrm{I} 2$ & $-339.54 \pm 0.79$ & $+50.48 \pm 0.36$ & +0.26 & -0.01 \\
31 & $\mathrm{~K} 2$ & $-250.15 \pm 1.02$ & $+51.88 \pm 0.31$ & +0.16 & +0.04 \\
32 & $\mathrm{~J} 2$ & $-248.92 \pm 0.63$ & $+150.19 \pm 0.47$ & +0.14 & -0.18 \\
33 & $\mathrm{M} 2$ & $-155.22 \pm 0.63$ & $+48.98 \pm 0.21$ & -0.17 & +0.02 \\
34 & $\mathrm{~L} 1$ & $-149.37 \pm 0.42$ & $+146.76 \pm 0.38$ & +0.21 & -0.17 \\
35 & $\mathrm{~J} 1$ & $-151.83 \pm 0.51$ & $+247.22 \pm 0.58$ & +0.19 & -0.20 \\
36 & $\mathrm{I} 1$ & $-50.48 \pm 0.45$ & $+339.42 \pm 0.42$ & -0.17 & -0.07 \\
37 & $\mathrm{~K} 1$ & $-51.69 \pm 0.31$ & $+247.46 \pm 0.59$ & +0.05 & -0.41 \\
38 & $\mathrm{M} 1$ & $-49.96 \pm 0.08$ & $+151.33 \pm 0.57$ & +0.05 & +0.06 \\
39 & $\mathrm{~N} 1$ & $-51.13 \pm 0.34$ & $+52.72 \pm 0.30$ & +0.05 & +0.13 \\
\hline \hline & & & & & \\
\hline
\end{tabular}

Table 4.2: Average NCD positions in the $x y$-plane for all scans. Units are $\mathrm{cm}$ and relative to the PMT coordinates. The average uncertainty on each coordinate is about $0.5 \mathrm{~cm}$. The tilts, given in degrees, were extracted from the February/06 data only and are all consistent with zero. Refer to Figure 2.2a. 


\section{Time Dependences}

The NCD positions and tilts were examined as a function of time in [100]. No significant time dependence of either the coordinates, uncertainties, or tilts were found. Therefore the NCD-phase average positions were extracted by performing a weighted average over all the scans.

\subsubsection{Average NCD Positions}

The weighted average results of the NCD coordinates are given in Table 4.2. These positions were used in the SNO geometry to simulate neutrino and calibration data in the NCD phase $[65,100]$. The tilts, extracted with good precision from the February/06 scan only, were all consistent with zero hence the NCDs were assumed to be parallel to the $z$-axis.

The NCD positions have been measured using the OCA data taken for most NCD-scans although they were not always optimized for this measurement. The NCD positions were updated on a scan-by-scan basis to remove the shadowed PMTs from each scan consistently. This increased the efficiency to remove the NCD shadows as explained in Section 4.4. It then led to data sets that had little attenuations caused by the absorption of photons on the NCDs.

\subsection{NCD Shadows and Reflections}

Knowing both the LB and NCD positions and their associated uncertainties, the effects of the NCDs in the PMT occupancy data were either removed or corrected for from the clean OCA data sets. This section describes how the NCD shadows and re- 
flections were accounted for in the OCA analysis, leading to an unbiased measurement of the optical constants.

\subsubsection{NCD Shadows}

The NCD shadows can be corrected for with good accuracy for a point source emitting C light [103]. In the case of distributed sources such as the LB, the accuracy of the correction varies with the position uncertainty and knowledge of the source light distribution. Therefore the NCD shadows were removed from the OCA PMT data rather than being corrected for.

The NCD shadow cut is purely geometrical. In addition to the positions and physical sizes of the LB, NCD, and PMT, the cut also depends on a parameter called the tolerance, $\Delta L$, which controls the acceptance of the cut.

\section{Shadow Tolerance Parameter}

The tolerance adds a radial distance to the physical radius of the NCDs to account for partial shadowing and position uncertainties. The value of $\Delta L$ cannot be lower than $2.579 \mathrm{~cm}$, the physical radius of the NCDs. The evaluation of the tolerance is necessary because of the uncertainties in the LB and NCD positions. The distribution of the NCD radial uncertainties, $\Delta r=\sqrt{\Delta \mu_{x}^{2}+\Delta \mu_{y}^{2}}$, where $\Delta \mu_{x}$ and $\Delta \mu_{y}$ are the NCD coordinate uncertainties, similar to those in Table 4.2, was obtained on a scanby-scan basis. Typical distributions of $\Delta r$ gave a mean of $2.2 \mathrm{~cm}$ and a spread of $0.3 \mathrm{~cm}[97,100]$. Thus, to take into account that some NCD position fits are worse than others, the spread was used to define a confidence level for the shadowed regions that allowed all 40 counters to be treated consistently. For typical scans, at the $3 \sigma$ 
level (99\% CL),

$$
\begin{aligned}
\Delta L & =\Delta r+3 \sigma_{\Delta r}+\Delta r_{\mathrm{LB}} \\
& =2.2+3 \times 0.3+2=5.1 \mathrm{~cm} .
\end{aligned}
$$

An average tolerance value of $\Delta L \sim 5 \mathrm{~cm}$ across all scans tagged and eliminated shadowed PMTs with high accuracy.

\section{Geometrical Cut}

Figure 4.4 shows how the NCD shadow cut works and the resulting pattern of the tolerance parameter. This geometrical cut flags PMTs associated with the paths crossing the regions in a radius $\Delta L$ centered on the fitted NCD positions. All NCDs are checked for every run. A PMT is flagged if at least one NCD fulfills the shadow requirement.

Figure $4.4 \mathrm{~b}$ shows the results of applying the shadow cut for two tolerance values. The fraction of PMTs removed when the LB is at the center corresponds to $55 \%$ and $71 \%$, for $\Delta L=2.579$ and $5 \mathrm{~cm}$, respectively. When the LB was outside the NCD array, the numbers changed to $37 \%$ and $41 \%$. The cut removed more PMTs within the NCD array because of the overlap between the shadow patterns. The overlap of the cut from two runs affects the statistics drastically [84]. For example, the cut removed $81 \%$ of PMTs from the overlap of the two previous LB positions with $\Delta L=5 \mathrm{~cm}$. This is the effect that motivated the use of the Occupancy-Efficiency method over the Occupancy-Ratio method (as discussed in Section 3.2.2). 


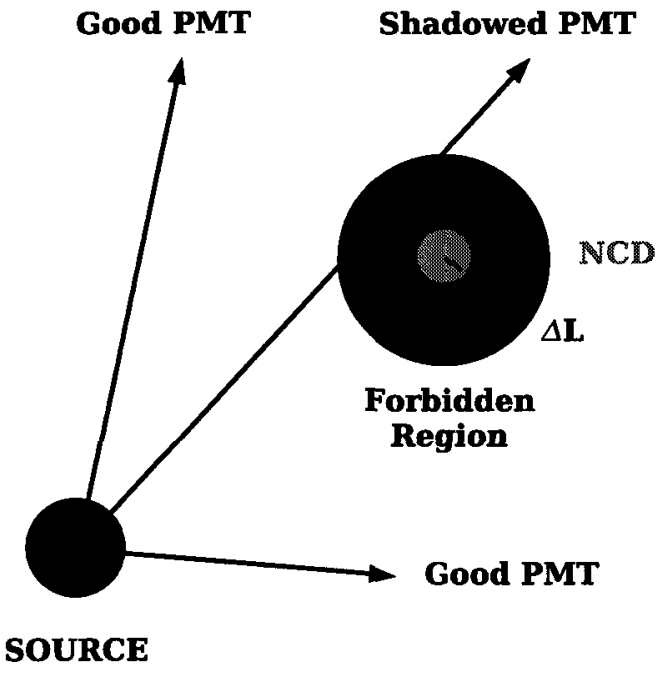

(a) Identification of shadowed PMTs.

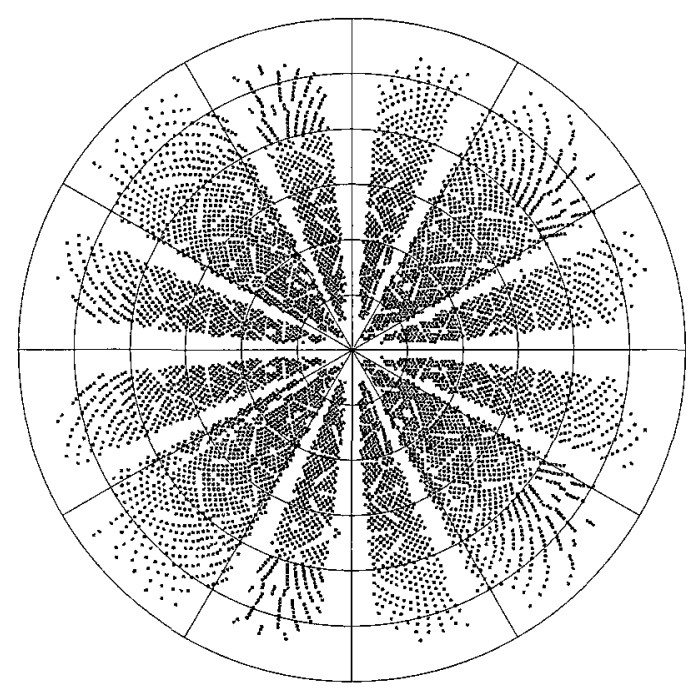

(b) Shadowed PMTs.

Figure 4.4: (color) Effect and pattern of the NCD shadow cut. (a) The geometry is used to identify shadowed PMTs. The tolerance defines a cylinder of radius $\Delta L$ with axis parallel to the detector $z$-axis around the NCDs. (b) Polar projection map of the PSUP showing the result of applying the cut with the LB at the center of the detector. Different shades of color show the cumulative effect of the cuts obtained with tolerance $\Delta L$ of $2.579 \mathrm{~cm}$ (red) and $5 \mathrm{~cm}$ (red and blue).

\subsubsection{NCD Reflections}

After the NCD shadows were removed, all that was left to affect PMT occupancies were the reflections off the NCD surfaces. The NCD reflections (NCDRs) account for a small fraction of the PMT counts thus they must in principle also be removed to ensure an unbiased measurement of the media attenuations. Considering the effect is small, there are two reasons why a correction was applied on the PMT data rather than using a cut. First, due to the diffuse nature of the NCDRs, the calculation of the reflection paths from the LB to the NCDs and then to the PMTs becomes impractical. Second, the LB was often near the NCDs and a cut would have flagged almost all PMTs for some runs. Therefore, a correction was used instead to preserve 


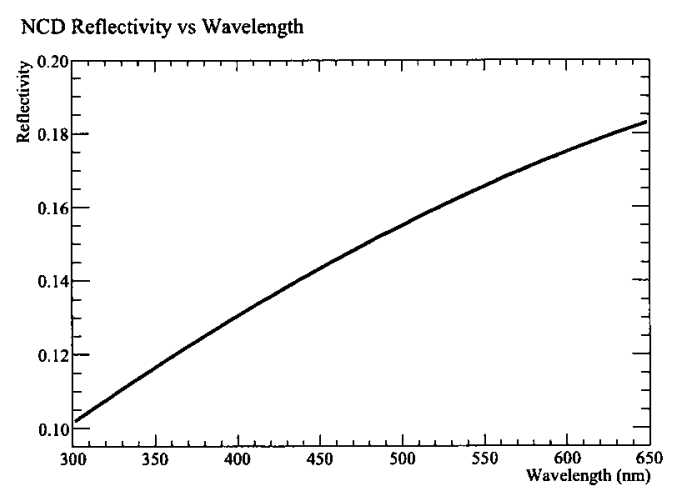

(a) NCD reflectivity $R(\lambda)$.

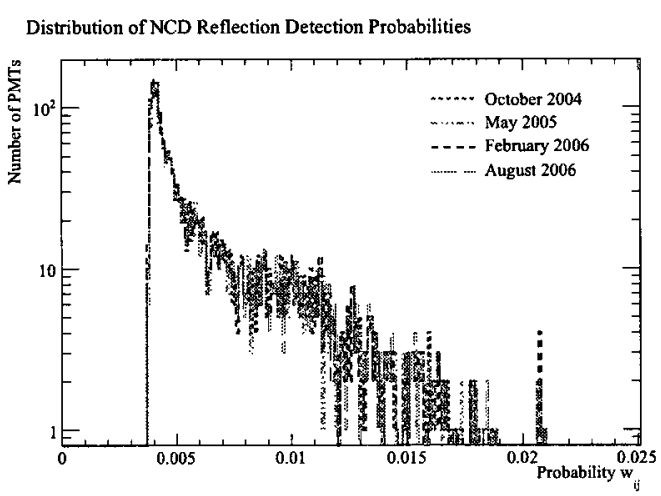

(b) Probability $w_{i j}$ for central runs.

Figure 4.5: Quantities involved in the PMT occupancy correction due to NCD reflections.

a good level of statistics in the fit.

The technique consists in evaluating the acceptance probability, $w_{i j}$, of photons leaving the source $i$, reflecting on all NCDs, and hitting a PMT $j$ within the analysis time window. The details of the calculation of $w_{i j}$ can be found in Subsection A.1.2 of Appendix A.

\section{NCD Reflectivity}

The probability $w_{i j}$ depends on the NCD reflectivity [70],

$$
R(\lambda)=-0.01387+4.5357 \times 10^{-4} \lambda-2.3154 \times 10^{-7} \lambda^{2},
$$

where $\lambda$ is the wavelength in $\mathrm{nm}$, plotted in Figure 4.5a. Note that the parameters in the reflectivity function have unknown errors. However independent measurements have shown that the spread in the reflectivity data is about $1.5 \%$ [104]. Figure $4.5 \mathrm{~b}$ shows the distribution of $w_{i j}$ for the central runs of many scans. The distributions look alike because $w_{i j}$ depends strongly on the geometry. The resulting reflection 
probabilities are low, ranging from $0.5 \%$ to $1.5 \%$, mainly due to the narrow time window ( $\pm 4 \mathrm{~ns})$ of the OCA analysis.

\section{Correction of PMT Occupancies}

The probability $w_{i j}$ was then used to correct the observed occupancy $O_{i j}^{\text {raw }}$ by adding a term to the occupancy correction formula that already corrects for multi photoelectron (MPE) effects. The corrected occupancy, $\mu_{i j}$, was obtained numerically from the NCD-phase occupancy correction formula described in detail in Section A.1 of Appendix A:

$$
O_{i j}^{\text {raw }}=1+\left(\mu_{i j} w_{i j}-1\right) \times e^{-\mu_{i j}}
$$

For a typical value of $O_{i j}^{\text {raw }}=0.03$, the MPE correction alone $\left(w_{i j}=0\right)$ results in an increases of the occupancy by $1.53 \%$, to 0.03046 . For the same value of $O_{i j}^{\text {raw }}$, and using $w_{i j}=0.005$, the combined correction results in a value of 0.03031 , a $1.03 \%$ increase. In general the overall correction applied to most PMT occupancies was of the order of $1-2 \%$.

\subsubsection{Changes in the NCD Phase}

The optical effects due to the presence of the NCDs in the detector are unwanted in the OCA data sets. The NCD partial shadows were removed using the fitted LB and NCD positions and a tolerance parameter. The cut is purely geometrical and removed the shadowed PMTs from the data with a high confidence level.

The NCD reflections are irreducible because of their diffuse nature. The PMT occupancies were corrected to take into account a small contamination in the signal time window. 
After the corrections of these effects the size of the OCA data set was significantly smaller but ready for a clean measurement of the optical model parameters. Section 4.5 presents the measurements of the model parameters in the NCD phase.

\subsection{Optical Parameters for the NCD Phase}

This section presents the optical constants for the NCD phase, obtained with the PMT efficiencies measured in the NCD commissioning phase and applying them with the Occupancy fit method to the scans of Table 4.1. The total errors given with the measured parameters include the contribution of systematic uncertainties, detailed in Section A.2 of Appendix A.

\subsubsection{Data Set and Selection}

Five scans were selected from Table 4.1: October/04, February/05, May/05, February/06, and August/06. There were known problems with the October/05 and June/06 scans, which made those data difficult to analyze. The five selected scans however cover the whole time range of the NCD phase and allowed for the monitoring of the time dependence of the optical constants. For comparisons, there was only one full scan in the $\mathrm{D}_{2} \mathrm{O}$ phase [56], and five scans in the salt phase [105].

\section{Run and PMT Selection}

In rare cases, runs were rejected from the run lists when the occupancy data were corrupted, path calculations failed, source position fit was incompatible with the manipulator position, or when there were known issues with the laser system at the 
moment the data were taken.

Otherwise, the processed data were selected using two classes of cuts: the first class is based on the geometry and light paths, and the second is an implicit $\chi^{2}$ cut. Figure $4.4 \mathrm{~b}$ showed the NCD shadow geometrical cut for a central run. To this figure should be added the convolution of the other cuts, such as kevlar ropes, belly plates, neck AV, and NCD anchors at the bottom of the AV. These cuts are referred to as geometrical cuts. For a central run, these cuts remove roughly $70 \%$ of the PMTs, the largest fraction being due to the NCD shadows. The $\chi^{2}$ cut is different and performed at run-time while the fitter minimized the $\chi^{2}$.

\section{Statistics}

Table 4.3 details the statistics before and after the selection cuts. The statistics in 'Passed' are obtained after applying the most restrictive $\chi^{2}$-cut of $\chi^{2}<25$. The fraction of the PMTs that passed all cuts is about $25 \%$ in the NCD phase, compared to $65 \%$ in the commissioning phase. The largest contribution for this drop in the statistics came from the NCD shadows removal, and as a consequence, the number of PMTs removed by the $\chi^{2}$-cuts is lower in proportion.

\subsubsection{Results}

The main figure of merit is the $\chi^{2}$ of Equation (3.6) returned by Levenberg-Marquardt minimizer [106]. Typical reduced $\chi^{2}$ values were of the order of [1-1.2] for the number of degrees of freedom in Table 4.3 divided by six minus the $>150$ fitted parameters. A $\chi^{2}$-pull analysis [97] showed no evidence of correlations between extreme $\chi^{2}$-values and paths, flags, or occupancy corrections. 


\begin{tabular}{|l|c|c|c|c|}
\hline \hline Scan & Total & Geometrical Cuts & $\chi^{2}$ Cuts & Passed \\
\hline \hline Commissioning & $1,787,076$ & $424,186(24 \%)$ & $213,713(12 \%)$ & $1,149,177(64 \%)$ \\
\hline October 2004 & $2,071,577$ & $1,431,480(69 \%)$ & $129,086(6 \%)$ & $508,522(25 \%)$ \\
February 2005 & $2,132,269$ & $1,483,886(70 \%)$ & $131,575(6 \%)$ & $516,808(24 \%)$ \\
May 2005 & $2,187,092$ & $1,503,878(69 \%)$ & $134,903(6 \%)$ & $548,311(25 \%)$ \\
February 2006 & $1,909,550$ & $1,333,379(70 \%)$ & $123,739(6 \%)$ & $452,432(24 \%)$ \\
August 2006 & $2,646,727$ & $1,758,174(66 \%)$ & $146,121(6 \%)$ & $742,432(28 \%)$ \\
\hline \hline
\end{tabular}

Table 4.3: Statistics, in number of PMTs, available for the extraction of the optical constants throughout the NCD phase. The maximum number of PMTs per run is roughly 9,000 . The numbers are integrated over the six wavelengths of the laser for a given scan. The $\chi^{2}$ cut also includes a fraction of the PMTs without measured efficiencies. Note that the NCDs were not in the detector for the commissioning phase scan.

\section{Media Extinctions}

The terminology here is the same as in Chapter 3, where attenuations, attenuation coefficients, and inverse attenuation lengths refer to the same physical quantity. The media attenuation coefficients in Equation (3.1) in SNO are total extinctions composed mainly of the pure media attenuation part. A small fraction of the photons experience forward Rayleigh scattering and are not detected by the PMTs within the time window. Therefore the pure attenuation lengths were obtained by subtracting a given fraction of the theoretical Rayleigh scattering lengths from the measured total extinction lengths (see Section A.3 of Appendix A).

Figure 4.6 shows the $\mathrm{D}_{2} \mathrm{O}$ and $\mathrm{H}_{2} \mathrm{O}$ attenuations as a function of wavelength. Figure $4.6 \mathrm{a}$ shows that on average the $\mathrm{D}_{2} \mathrm{O}$ attenuations are consistent but slightly lower than the values measured in October/03 during the commissioning phase. For reasons that are explained in Section A.2, the errors on the attenuations are larger on a scan-by-scan basis due to the new NCD systematic errors. Similar conclusions 
apply to the $\mathrm{H}_{2} \mathrm{O}$ attenuations in Figure 4.6b. For most wavelengths, the attenuations extracted in the NCD phase were consistent with the values measured in the commissioning phase, within the uncertainties. Tables A.4 and A.5 of Appendix A give the numerical values of the extracted attenuations with total uncertainties for all the scans and wavelengths.

\section{PMT Angular Response}

Figure 4.7 shows the PMT relative angular response for the six wavelengths. The smoothed curves are interpolation points drawn between the NCD commissioning and NCD-phase average response functions. The response measured in the NCD phase was lower than it was in the NCD commissioning phase. Considering the error bars the values at low angles were consistent, but the response at large angles between $20^{\circ}$ and $40^{\circ}$ was about $4 \%$ lower. The most likely physical explanation is the degradation of the PMT reflectors [107] that converts specular into diffuse reflections making the concentration of light onto the photocathode less efficient.

\section{Laserball Distribution and Mask Function}

The LB intensity is modeled as a spherical source with intensity distributed in bins of $\left(\cos \theta_{\mathrm{LB}}, \phi_{\mathrm{LB}}\right)$ weighted by a function that describes the shadowing due to the source carriage and stainless steel body that depends on $\cos \theta_{\mathrm{LB}}$ only. It accounts for the largest number of parameters in the model. There are currently two parameterizations, discussed and compared in [108]; the simplest was used in the NCD phase, which assumed that the LB isotropy was uniform.

Figure $4.8 \mathrm{a}$ shows the LB distribution as a function of the direction $\left(\cos \theta_{\mathrm{LB}}, \phi_{\mathrm{LB}}\right)$. 
D2O Inverse Attenuation Length vs Wavelength

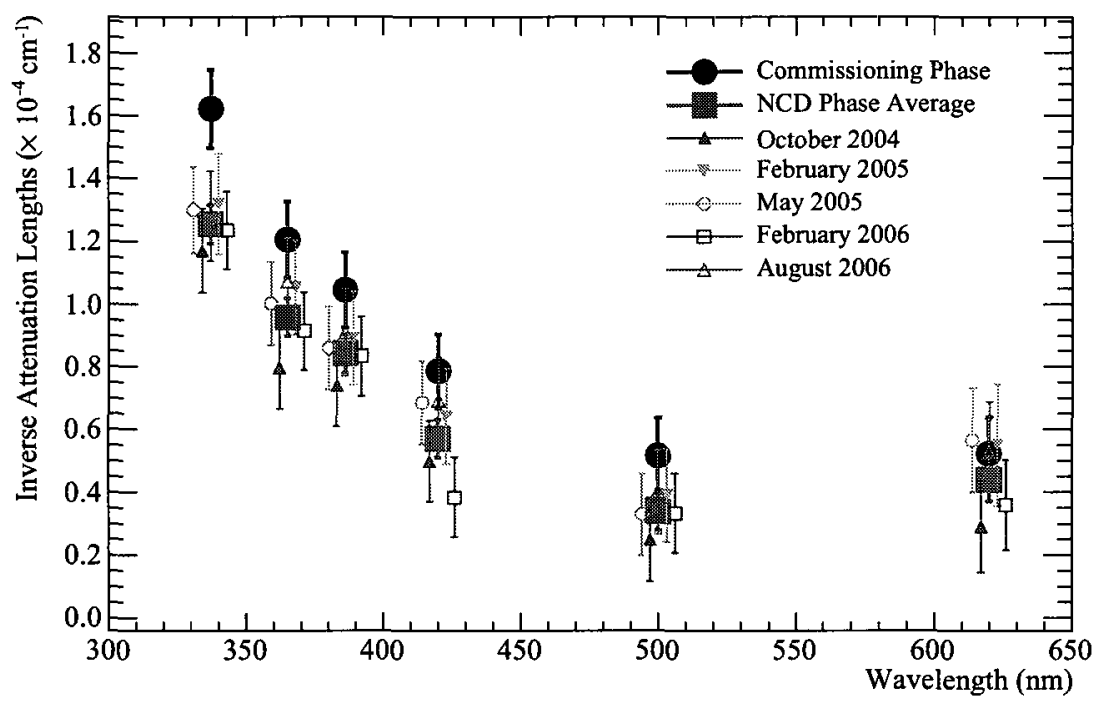

(a) $\mathrm{D}_{2} \mathrm{O}$ total extinctions.

H2O Inverse Attenuation Length vs Wavelength

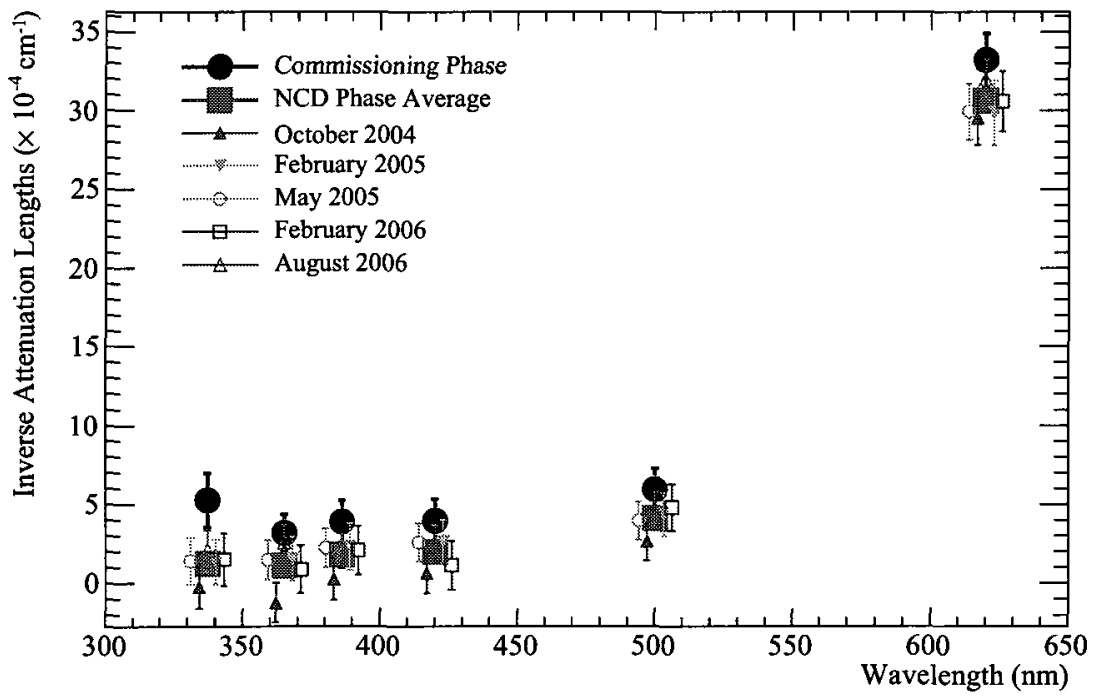

(b) $\mathrm{H}_{2} \mathrm{O}$ total extinctions.

Figure 4.6: Media inverse total extinction lengths as a function of wavelength in the NCD phase. Some points are shifted to see the error bars. 


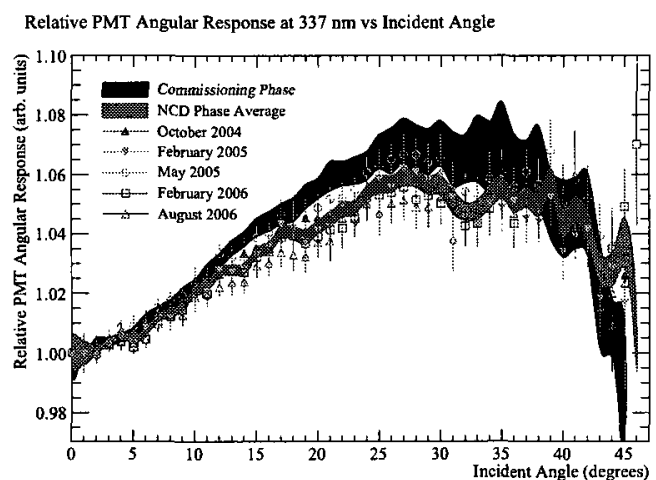

(a) $337 \mathrm{~nm}$.

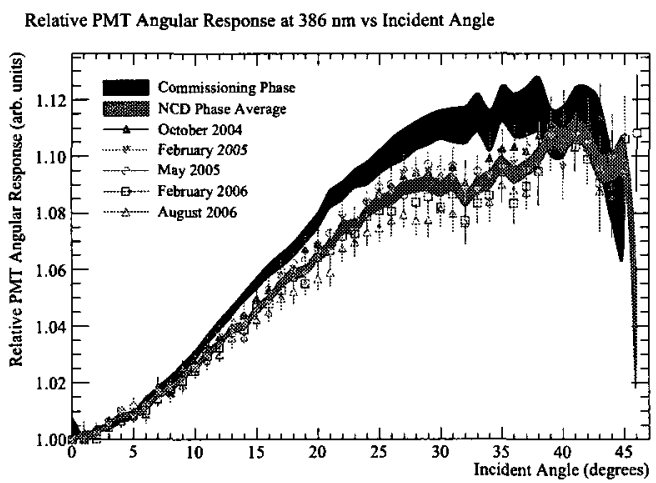

(c) $386 \mathrm{~nm}$.

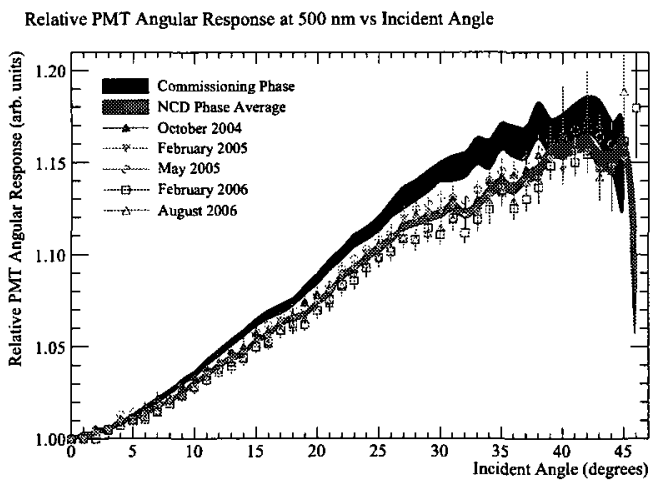

(e) $500 \mathrm{~nm}$.

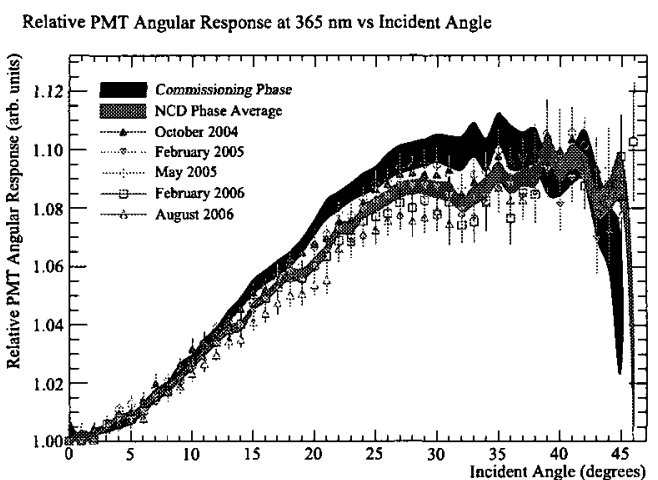

(b) $365 \mathrm{~nm}$.

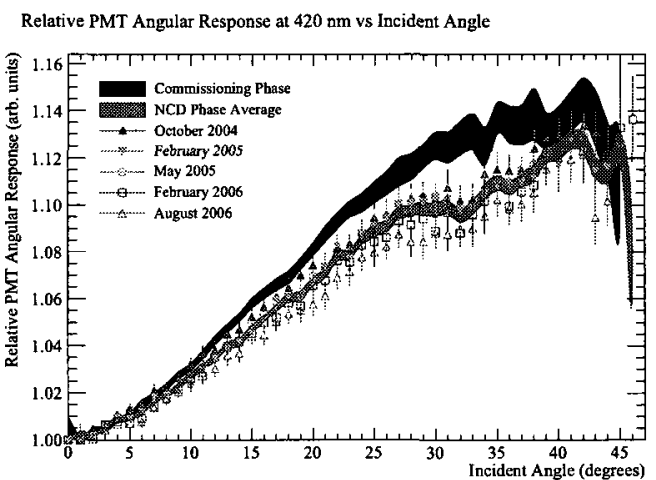

(d) $420 \mathrm{~nm}$.

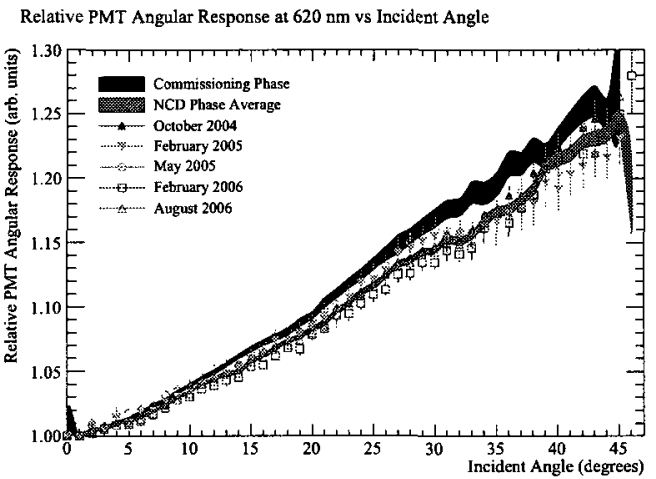

(f) $620 \mathrm{~nm}$.

Figure 4.7: PMT relative angular response in the NCD phase. The commissioning phase and NCD-phase average response functions are shown for comparison. Each response bin is one degree-wide. Note that the scale is different for each wavelength. 


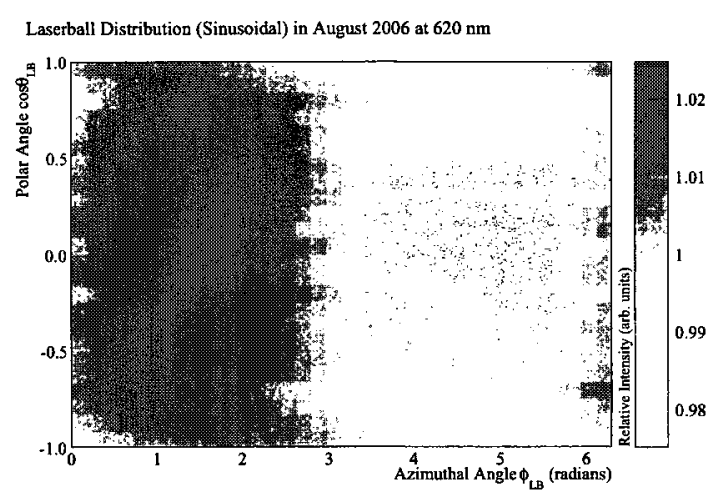

(a) August/06 distribution.

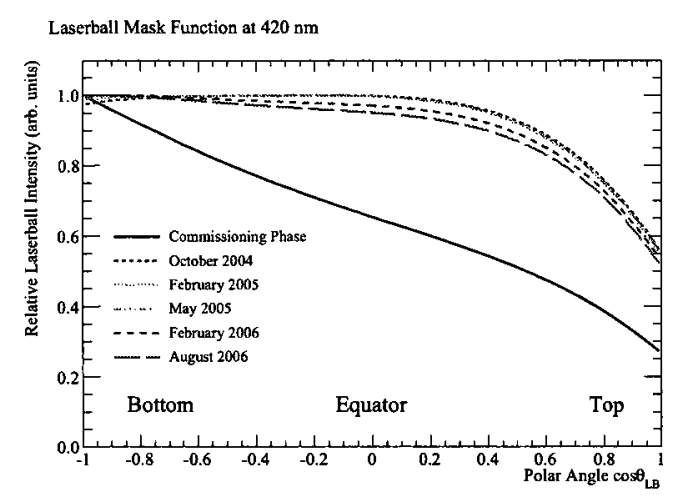

(b) Mask function.

Fiogure 4.8: Laserball relative intensity distribution and mask function in the NCD phase at $420 \mathrm{~nm}$. (a) Extracted at $620 \mathrm{~nm}$. (b) Extracted at $420 \mathrm{~nm}$ from the selected scans and compared to the commissioning phase function.

The relative half-amplitude is about $2.5 \%$ with almost no dependence on $\cos \theta_{\mathrm{LB}}$. The statistical uncertainties in the $24 \cos \theta_{\mathrm{LB}}$ amplitudes of the $\mathbb{L B}$ model was about $5 \%$ on average [108].

Figure $4.8 \mathrm{~b}$ shows the LB mask function as a function of $\cos \theta_{\mathrm{LB}}$ extracted at $420 \mathrm{~nm}$ from the selected scans. The commissioning-phase mask function is shown to demonstrate the improvement of the uniformity of the LB used during the NCD phase. These results all show a slight decrease of the intensity in the upper part of the detector compared to the bottom, confirming the shadowing effects from the source carriage apparatus. In general the isotropy of the LB was much better in the NCD phase compared to what it was in the commissioning phase since the $\mathbb{L B}$ diffuser was physically replaced by a new one at the beginning of the NCD phase.

\section{Tơme Deperdences}

Fortunately, the frequent LB scans tracked the time dependences of the media attenuations and PMTR. Figure 4.9 shows the time dependence of the $\alpha_{\mathrm{d}, \mathrm{h}}$ measurements. 


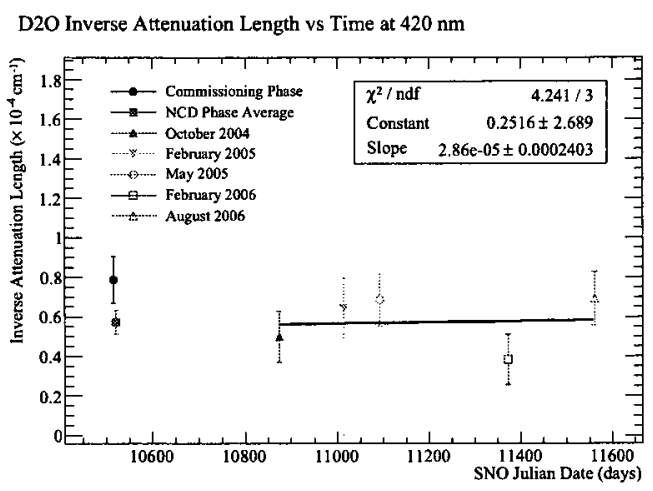

(a) $\mathrm{D}_{2} \mathrm{O}$ total extinctions.

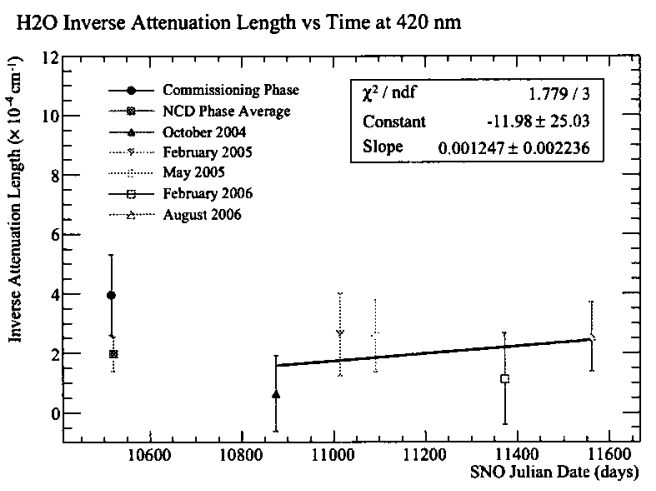

(b) $\mathrm{H}_{2} \mathrm{O}$ total extinctions.

Figure 4.9: Media inverse total extinction lengths as a function of time at $420 \mathrm{~nm}$.

In each case, a linear function was fitted to the results obtained in the period between October/04 and August/06, spanning the entire NCD phase. The slopes, characterizing the drifts of the attenuation lengths, were consistent with zero when the errors were taken into account. This was observed for all wavelengths in both media. Therefore the NCD-phase average values are representative of the whole NCD phase.

Similar checks were carried out for the PMT response. Although a 2-3\% trend was observed, the drifts were not significant given the uncertainties in each response bin. Similar to the attenuation lengths, the average PMT response was used for the entire NCD phase. Other parameters of the OCA model are not required to be checked because their effect on the physics is negligible.

\subsection{Uncertainties due to Optics}

The processing of events and production of $\mathrm{MC}$ simulations were accomplished by SNOMAN with the help of four input optics files: PMT efficiencies, media attenuation lengths, PMT angular response, and NCD positions. Sections A.3 and A.4 of 
Appendix A give the technical details of how some of these files are built at the end of the OCA chain.

In this section, the systematic uncertainties associated with the optical constants are briefly summarized in Subsection 4.6.1. From the latter, the systematic errors induced by the PMT efficiencies and NCDRs are propagated to the general event vertex position fitter, FTN [103], and energy estimator, RSP [58, 109]. The method is similar to the estimation of the OCA systematic errors, where distributions were compared with and without a given systematic shift to evaluate its impact. The systematic effects were introduced by loading separate systematically-shifted files into SNOMAN, and the comparison was made by comparing the results generated with the official files used throughout the NCD phase.

\subsubsection{Systematic Uncertainties of the Optical Constants}

The 16 systematic uncertainties affecting the optical parameters in the OCA analysis are described in detail in Section A.2 of Appendix A. The list of systematic uncertainties and their quantitative effects on the media attenuations and PMTR are summarized in Table 4.4. One of the conclusions of this chapter is that the systematic uncertainties of the new optical effects introduced with the NCDs mainly impact the precision of the $\mathrm{D}_{2} \mathrm{O}$ attenuation coefficients. The dominating components came mainly from the PMT efficiencies $(i=12)$ and NCDR probability $(i=16)$. The next subsections concentrate on the latter two systematic errors, and how they can affect solar neutrino analyses via position and energy reconstruction. 


\begin{tabular}{|c|l|r|}
\hline \hline$i$ & Systematic Error & Parameters affected (limit) \\
\hline 1 & Radial position scale & $\alpha_{\mathrm{d}}(\leq 10 \%), \alpha_{\mathrm{h}}(\leq 20 \%)$ \\
2 & Radial position shift & $\alpha_{\mathrm{d}}(\leq 15 \%), \alpha_{\mathrm{h}}(\leq 25 \%)$ \\
3 & Radial position smear & $\alpha_{\mathrm{d}}(\leq 5 \%), \alpha_{\mathrm{h}}(\leq 5 \%)$ \\
4 & Source $z$-position & $\alpha_{\mathrm{h}}(\leq 2.5 \%)$ \\
5 & Source $x$-position & Negligible \\
6 & Source size & $\alpha_{\mathrm{h}}(\sim 8 \%)$ \\
\hline 7 & LB amplitude & Negligible \\
8 & LB uniformity & Negligible \\
9 & PMT variability & $\alpha_{\mathrm{d}}(\sim 1 \%)$, PMTR $(\leq 1 \%)$ \\
10 & $\chi^{2}$ cut $(4 \sigma)$ & $\alpha_{\mathrm{d}}(\sim 2 \%), \alpha_{\mathrm{h}}(\sim 1 \%)$ \\
11 & $\chi^{2}$ cut $(3 \sigma)$ & $\alpha_{\mathrm{d}}(\sim 2 \%), \alpha_{\mathrm{h}}(\sim 1 \%)$ \\
\hline 12 & PMT efficiencies & $\alpha_{\mathrm{d}}(\leq 6 \%)$, PMTR $(\leq 1 \%)$ \\
13 & Response asymmetry & PMTR $(\leq 1 \%)$ \\
\hline 14 & Tolerance parameter & $\alpha_{\mathrm{d}}(\leq 1 \%)$ \\
15 & NCD reflectivity & $\alpha_{\mathrm{d}}(\leq 1 \%)$ \\
16 & Reflection probability & $\alpha_{\mathrm{d}}(\sim 5 \%)$ \\
\hline \hline
\end{tabular}

Table 4.4: Summary of the systematic uncertainties on the media attenuations and PMT relative angular response in the NCD phase.

\subsubsection{Effects on Vertex Reconstruction}

The FTN fitter is an upgraded version of FTU [110] for the NCD phase. FTN relied on a time-residual probability density function, determined with $\mathrm{MC}$, to reconstruct the position of an event. For each event, FTN took into account the NCD shadowing patterns using the detector geometry and assigned weights to the individual PMTs that registered light. Although shifts in the positions of the NCDs had a negligible effect on the positions reconstructed by FTN [97], a change in the PMT patterns themselves, from the variations of the PMT efficiencies and NCD reflections, could affect the reconstructed vertex and event direction. 
Figure 4.10 shows the FTN resolution of the $x$-coordinate obtained with MC mono-energetic electrons generated at 5 and $8 \mathrm{MeV}$ uniformly in the AV and in randomly distributed directions. The resolution function, $R(x)$, contains a normal distribution centered on the expected value of the coordinate, and exponential tails with a contribution controlled with the $\alpha_{e}$ parameter:

$$
R(x)=\frac{1-\alpha_{e}}{\sqrt{2 \pi} \sigma} \exp \left\{-\frac{1}{2}\left(\frac{x-\mu}{\sigma}\right)^{2}\right\}+\frac{\alpha_{e}}{2 \tau} \exp \left\{\frac{-|x-\mu|}{\tau}\right\}
$$

The parameters obtained from the shifted distributions were found to be consistent with the nominal parameters, generated with the standard optics settings. The estimated bias for individual vertex coordinates, due to the optics, was less than $0.1 \mathrm{~cm}$, negligible compared to FTN's own resolution of $15-20 \mathrm{~cm}$ [103]. Hence the two uncertainties studied and all uncertainties from OCAs were neglected in the vertex reconstruction and angular resolution of FTN in the NCD phase.

\subsubsection{Effects on Energy Estimation}

The RSP energy estimator was also modified for the NCD phase to take into account the NCD shadowing and reflection effects. Correction factors as a function of the position and direction of an event in the detector, derived from $\mathrm{MC}$, were applied so that events could be assigned an energy slightly higher (lower) if shadowing (reflections) occurred. The RSP energy scale and data tables were generated with the set of optical constants shown in Section 4.5 for the NCD phase.

The shifted optics banks were used to reconstruct the energy of MC mono-energetic electrons with RSP expecting the nominal optics settings. RSP had been configured 


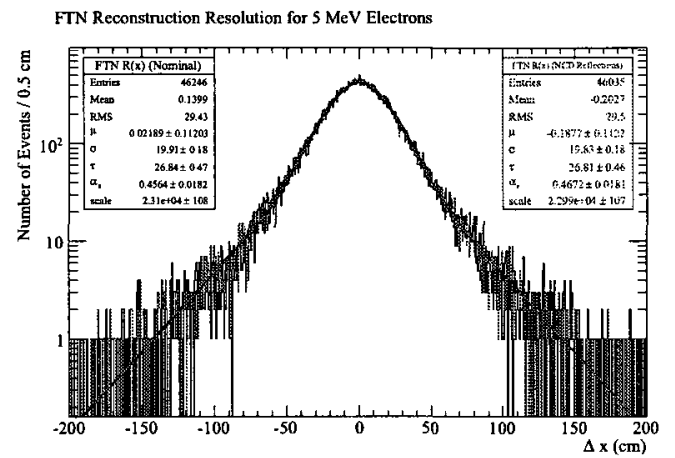

(a) Effect of NCD reflections at $5 \mathrm{MeV}$.

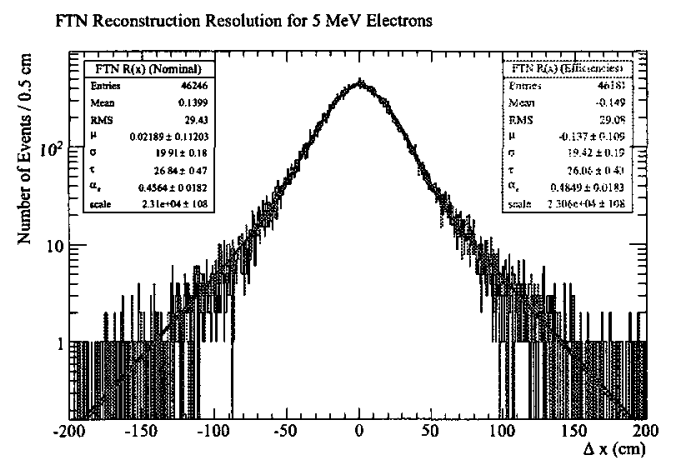

(c) Effect of PMT efficiencies at $5 \mathrm{MeV}$.

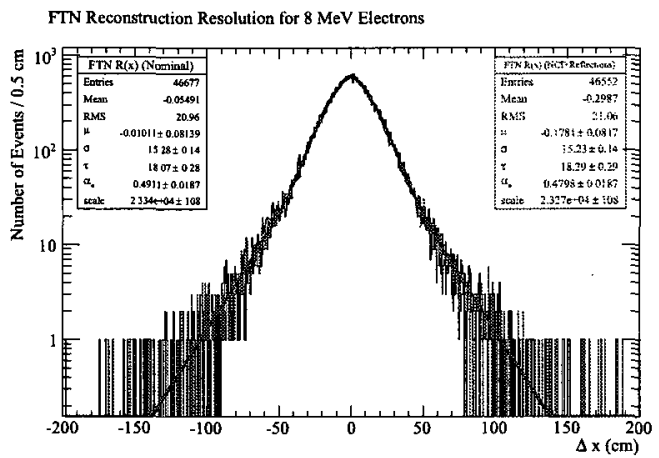

(b) Effect of NCD reflections at $8 \mathrm{MeV}$.

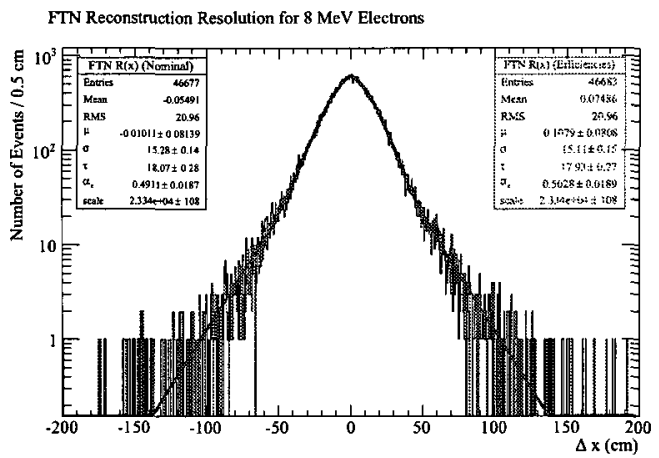

(d) Effect of PMT efficiencies at $8 \mathrm{MeV}$.

Figure 4.10: Effect of the new optics systematic errors on vertex reconstruction. The mono-energetic electrons are generated at 5 and $8 \mathrm{MeV}$, using an isotropic distribution in the $\mathrm{AV}$ volume.

to match the nominal constants such that estimating the optics effects this way made RSP and OCA inconsistent. Thus large shifts were expected. Figure 4.11 shows the RSP distribution of reconstructed electron kinetic energies as obtained from MC mono-energetic electrons. The mean energy and energy resolution obtained from the shifted distributions are consistent with the nominal distributions. The differences were of the order of $1.5 \%$ for the mean energy, and $1.8 \%$ for the resolution. Although these differences were large, it was expected that if RSP and the shifted optics were consistent these energy shifts would be much smaller [111]. Hence the effects of the 


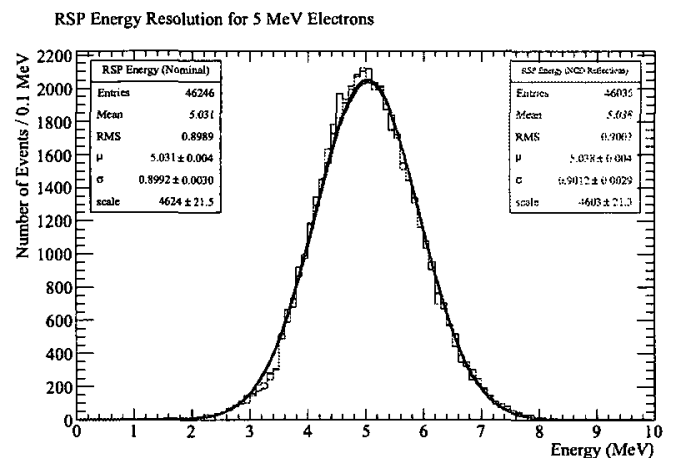

(a) Effect of NCD reflections at $5 \mathrm{MeV}$.

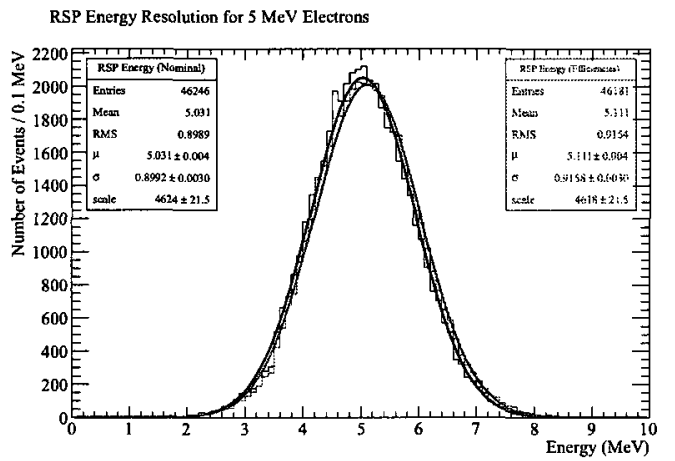

(c) Effect of PMT efficiencies at $5 \mathrm{MeV}$.

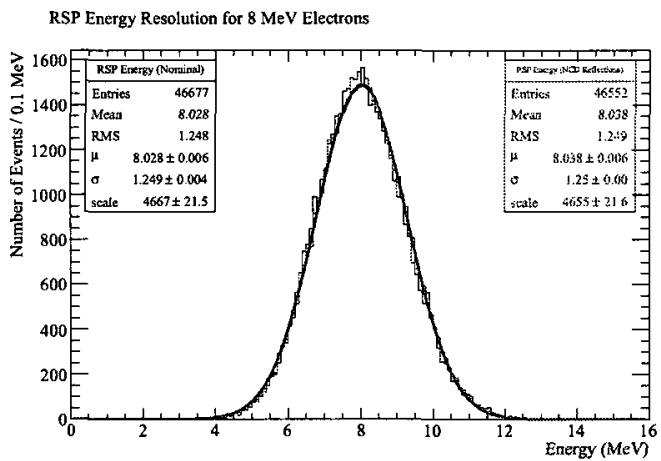

(b) Effect of NCD reflections at $8 \mathrm{MeV}$.

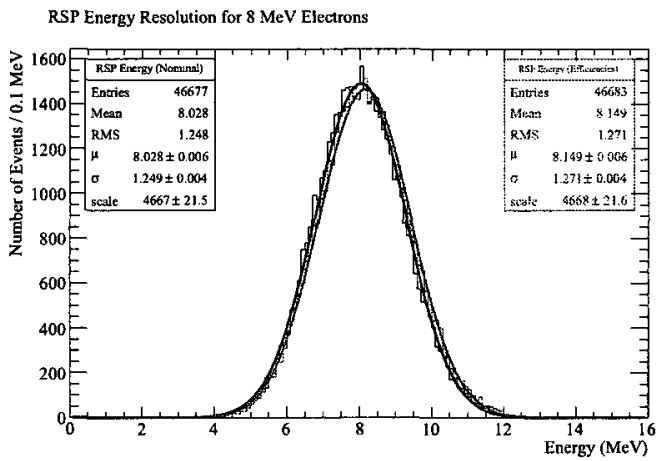

(d) Effect of PMT efficiencies at $8 \mathrm{MeV}$.

Figure 4.11: Effect of the new optics systematic errors on energy scale and resolution obtained with RSP with MC electrons. The mono-energetic electrons are generated at 5 and $8 \mathrm{MeV}$, using an isotropic distribution in the AV volume.

new optics uncertainties on RSP were evaluated to be less than $1 \%$ on both energy scale and resolution. The whole set of OCA systematic errors had an overall effect of about $1 \%$, comparable to previous phases of SNO [56].

\subsection{Conclusion on Optical Calibration}

Chapters 3 and 4 described the refinements and adaptations of the OCA analyses that were necessary to achieve comparable precision as in the $\mathrm{D}_{2} \mathrm{O}$ and salt phases of 
SNO. The work towards the NCD phase led to the understanding of the new detector response asymmetries that were then applied in the reprocessing of the SNO data and MC [35], and the work during the NCD phase provided calibration constants that were used directly in the first NCD-phase analysis [27].

In particular, the extraction of the PMT efficiencies allowed for a more realistic simulation of the detector response, but also contributed, with the Occupancy method, to keep the level of statistics in the OCA data sets to an acceptable level. The PMT statistics in the NCD phase decreased by a factor of $50 \%$ per scan mainly because of the uncertainties in the LB and NCD positions that constrained the NCD shadow to a large tolerance value. Combined with the effect of new NCD systematic effects, the errors in the media attenuations and PMTR increased compared to the previous phases, on a scan-by-scan basis. However, given the number of good scans and detector stability throughout the NCD phase, the overall uncertainties were decreased by calculating the weighted averages of the main optical parameters.

The media attenuations were stable across the NCD phase which eliminated the need to use a drift function, as in the salt phase [30]. The PMT angular response decreased by about $4 \%$ on average at large angles throughout the NCD phase. Other parameters of the model such as the isotropy of the LB source were stable. The PMT efficiencies could not be monitored in the NCD phase. An additional calibration set after the NCD phase (and the removal of the NCDs) would have provided an excellent check of their stability, but unfortunately it was not possible given the constraints of the SNO experiment schedule [112].

Finally, the changes brought to the OCA analysis for the NCD phase resulted in specific systematic uncertainties. The effects of the NCD shadows and reflections, 
and the PMT input efficiencies were added in quadrature to the pre-NCD systematic errors that had been already studied. The systematic uncertainties dominated the total uncertainty on the media attenuations, and contributed to a $1.5 \%$ error on the PMTR measurements. Among the new effects, the NCD reflectivity and input efficiencies were the ones that affected the optical parameters the most. The latter were studied further by inducing shifts on the input constants to the FTN and RSP fitters, responsible to provide the vertex position and event energy for each event in the NCD phase. The shifted reconstructed distributions of MC electrons did not show, in general, large shifts when compared to the nominal distributions. The NCD systematic errors on the optical constants did not affect the position and energy estimators by more than their inherent resolution.

The next chapters resume the main discussion of investigating the low energy neutrinos in SNO towards a precise measurement of the solar neutrino survival probabilities and oscillation parameters. Particularly relevant to OCAs, the signal extraction procedure introduced in Chapter 5 exploits the MC simulation improvements and neutrino event observables in a pattern recognition algorithm. 


\section{CHAPTER 5}

\section{Extraction of the SNO Signals}

The signal extraction (SigEx) procedure is the most important step of the data analysis chain leading to the solar neutrino flux measurements. The physics arising from the CC, ES and NC reactions of Equations (1.17) is extracted through a statistical separation technique that requires prior knowledge of the signal distributions as a function of a set of observables. This chapter explains the formalism of the SigEx procedure, lists the inputs, and presents the results of the solar neutrino fluxes measured at a low energy analysis threshold of $3.5 \mathrm{MeV}$ with the data of the first two phases of SNO.

\subsection{The Low Energy Threshold Analysis}

The low energy threshold analysis (LETA) is the latest analysis of the SNO data sets with a lowered observed energy threshold of $T_{\text {eff }}^{0}=3.5 \mathrm{MeV}$. The main motivation for the LETA is the inclusion of signal events that were unused in previous SNO publications, leading to an improvement of the sensitivity to low energy $\nu$ 's. Figure 5.1 supports the latter two aspects of the motivation by showing the energy distributions of the CC, ES and NC signals. Figure 5.1a shows the expected signal shapes with respect to the observed kinetic energy, $T_{\text {eff }}$, obtained from the MC simulation of SNO. 
Also shown is an analysis threshold of $5.5 \mathrm{MeV}$. The fraction of events within the range $T_{\text {eff }} \in[3.5,5.5] \mathrm{MeV}$ corresponds to approximately $20 \%, 35 \%, 45 \%$, and $25 \%$ of the total integral for $\mathrm{CC}, \mathrm{ES}, \mathrm{NC}$ in the $\mathrm{D}_{2} \mathrm{O}$, and $\mathrm{NC}$ in the salt phase, respectively. Figure $5.1 \mathrm{~b}$ shows the signal shapes or the effective response in neutrino energy space, $E_{\nu}$, for CC and ES events. The sensitivity to $\nu$ 's is enhanced in the range between 4 and $12 \mathrm{MeV}$, mainly due to $\mathrm{CC}$ events, and is maximal near $E_{\nu}=9.5 \mathrm{MeV}$.

In addition, the $\mathrm{D}_{2} \mathrm{O}$ and salt phases are combined such that the two different detector configurations are taken into account in the simultaneous measurement of the total NC flux and CC and ES spectra. Therefore the gain in statistics is significant in the LETA. The challenge of lowering the energy threshold is a careful analysis of all sources of background events (BGs) that can potentially misrepresent signal events. This section presents the main features of the LETA that was carried out by many SNO collaborators including the author. The technical details of the analysis can be found in $[35,80,83,113,114]$.

\subsubsection{Observables and Combined Analysis}

The richness of SNO has one unavoidable downside: individual events cannot be tagged to be of type CC, ES, NC, or BGs. Therefore the main goal of SigEx is to assign probabilities to all events based on their observables and expected signature in order to perform a statistical separation of each class of event. The observables were carefully chosen to optimize the discriminating power between the signal events and BGs that are simultaneously extracted from the clean neutrino data. This subsection describes the observables that were used in the present and past analyses of the SNO data. 
SNO Observed Energy Response

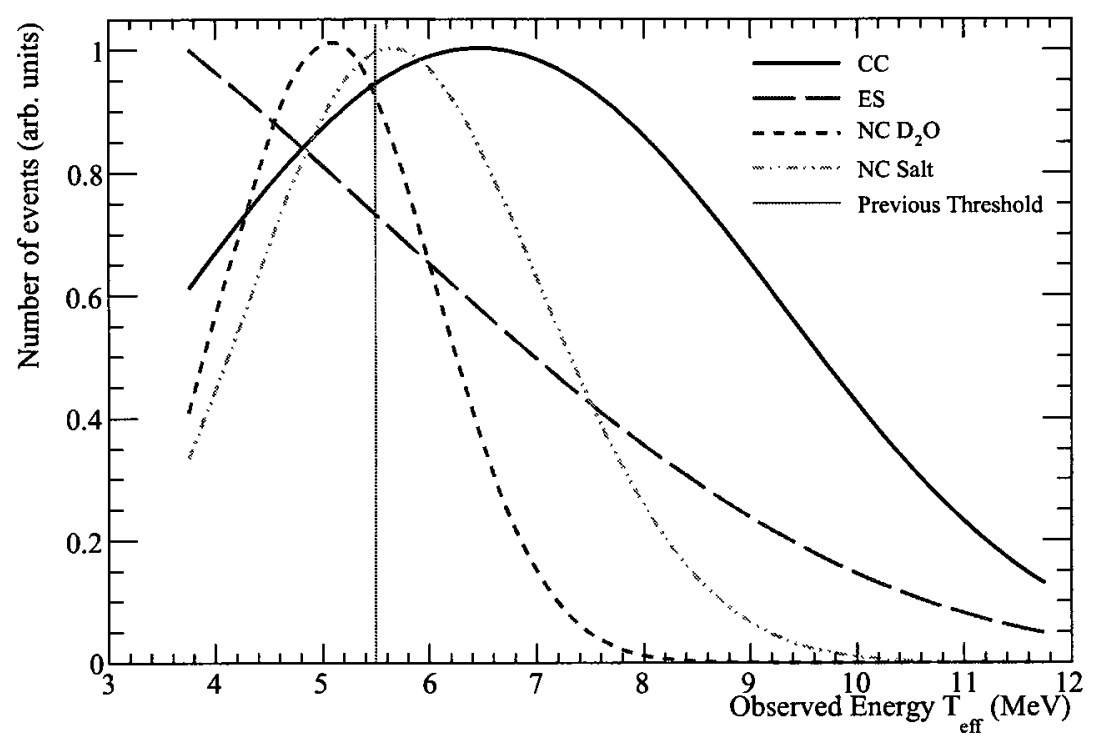

(a) Signal shapes as a function of reconstructed energy $T_{\text {eff }}$.

SNO Neutrino Energy Response

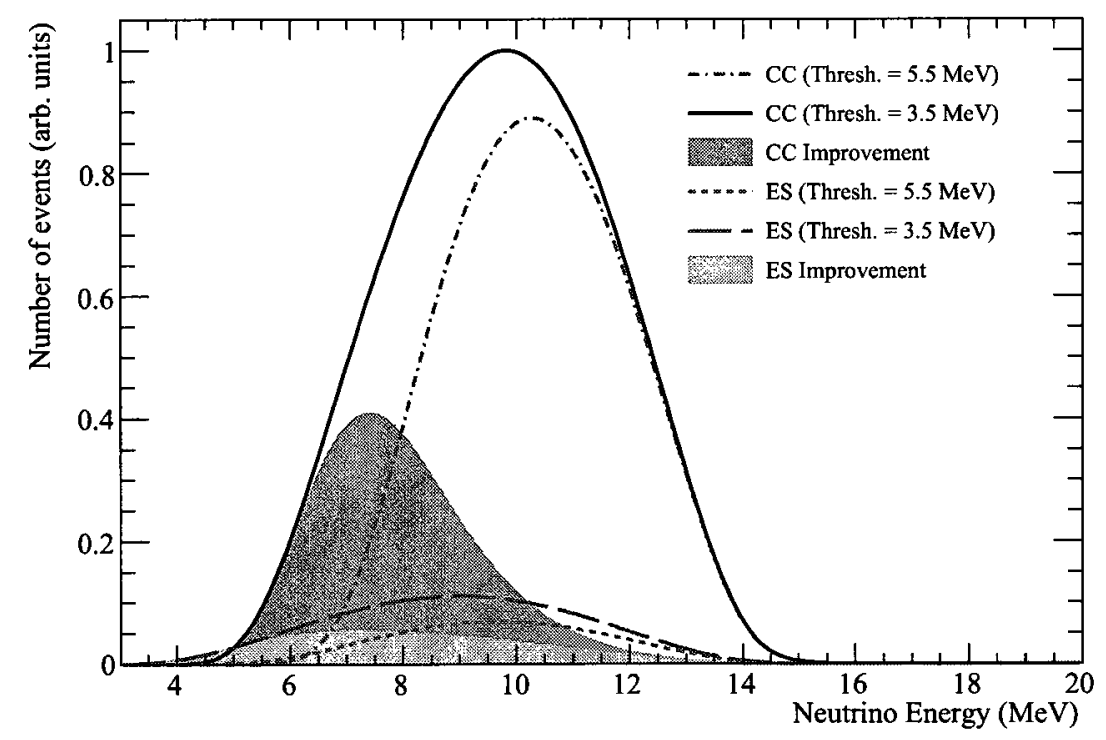

(b) Signal shapes as a function of neutrino energy $E_{\nu}$.

Figure 5.1: Expected SNO signal shapes due to solar neutrinos in the low energy threshold analysis. (a) The maximum of each shape is set to one. A previous threshold of $5.5 \mathrm{MeV}$ from the salt phase is shown [30]. (b) CC and ES distributions in $E_{\nu}$-space with the relative statistical improvements due to a lower threshold. The ES curve is scaled with respect to $\mathrm{CC}$ as a consequence of the differences between the reaction cross-sections. 


\section{Energy: $T_{\text {eff }}$}

The most important quantity is the effective electron kinetic energy, $T_{\text {eff }}$, reconstructed for each event based on the number of hit PMTs. In the LETA, the $\mathrm{D}_{2} \mathrm{O}$ and salt-phase events were assigned an energy with the estimator FTK [83] instead of RSP [58] (c.f. Chapter 4). FTK makes use of the scattered and reflected light which results in an improvement of the resolution in $T_{\text {eff }}$ by approximately $5 \%$.

The energy shape of each signal class can be constrained or unconstrained in SigEx. In both cases the relative fluxes for NC, CC, and ES reactions are extracted. A constrained analysis assumes a fixed energy shape, as derived from $\mathrm{MC}$, and only allows the normalization to vary. Oppositely, an unconstrained analysis is performed by letting the relative amplitudes of the pre-determined bins of $T_{\text {eff }}$ vary. In the following, the NC energy shape is constrained because the detector response to neutrons is well known and does not depend on the electron neutrino survival probability. On the other hand, the CC and ES spectra are unconstrained, allowing the effect of the survival probability to be extracted from the possible spectral distortions with respect to the unoscillated energy shapes.

\section{Radius: $\rho$}

In the LETA, the $\mathrm{D}_{2} \mathrm{O}$ - and salt-phase events were assigned a position in the detector with FTP [115] instead of FTU/FTN $[103,110]$ (c.f. Chapter 4). The FTP algorithm obtains the coordinates and direction of the events from the PMT positions and times. Vertex and direction reconstruction is imperative to calculate the three remaining observables: radial position, isotropy and direction with respect to the Sun.

The approximate spherical symmetry of the detector allows one to transform the 
position $(x, y, z)$ of each event into the radial position $r$, normalized by the radius of the AV and volume weighted: $\rho \equiv(r / 600.5)^{3}$. The radius observable is important in the separation of events that occured in the $\mathrm{D}_{2} \mathrm{O}(\rho<1)$ as opposed to those that occured in the $\mathrm{H}_{2} \mathrm{O}(\rho>1)$ that are mainly backgrounds.

\section{Isotropy: $\beta_{14}$}

The isotropy of an event is evaluated using the distribution of hit PMTs from the event position. The definition used in this analysis is

$$
\begin{aligned}
\beta_{14} & \equiv \beta_{1}+4 \beta_{4} \\
\beta_{l} & =\frac{2}{n(n-1)} \sum_{i=1}^{n-1} \sum_{j=i+1}^{n} P_{l}\left(\cos \vartheta_{i j}\right),
\end{aligned}
$$

where $n$ is the number of hit PMTs, $P_{l}$ are the Legendre polynomials, and $\vartheta_{i j}$ is the angle between the PMTs $i$ and $j$ from the vertex. The isotropy mainly aids to separate $\mathrm{NC}$ and $\mathrm{CC}$ events in the salt phase [30], and was also used in the $\mathrm{D}_{2} \mathrm{O}$ phase for consistency in the combined analysis.

\section{Event Direction: $\cos \theta_{\odot}$}

The direction of an event with respect to the Sun is calculated from the relative positions of the Sun and event within the detector. The angle with respect to the Sun-detector axis is $\theta_{\odot}$ and the observable is $\cos \theta_{\odot} \in[-1,1]$. It mainly separates ES events from other classes of events because of their strong angular dependence with the direction of the incoming $\nu$ 's. Backgrounds were assumed to have a uniform distribution in $\cos \theta_{\odot}$. 


\section{Combining Data Sets}

The consistency in the assignment of the observables in both the $\mathrm{D}_{2} \mathrm{O}$ and salt phases allowed for the extraction of the neutrino fluxes from both sets simultaneously. Each phase has its own set of MC-determined shapes of the observable distributions for signals and BGs, embedding the condition of operations and time dependence of the detector response into the analysis. Each probability density function (PDF) contributes to the common extraction of the solar neutrino flux as a function of $T_{\text {eff }}$. As a consequence of this construction, the individual NC, CC, and ES fluxes, that are measured in two different detector configurations, are constrained to agree with each other.

\subsubsection{Treatment of Backgrounds}

Backgrounds represent a significant fraction of the events in the data at low $T_{\text {eff }}$ and

for $\rho \gtrsim 1$. For each source of BG, the neutron- and electron-like events are treated as a unique type because the nuclear decays from which they originate result from the same decay chains. The BGs are categorized as additional classes of events, and thus MC-determined shapes in all observables are needed to discriminate them from the neutrino signals. In cases where BGs and signals have almost identical shapes, their scale are constrained from independent ex situ measurements (c.f. Table 2.3) to limit their reach over neutrino physics signals. Furthermore, the BGs are duplicated for each phase, since the detector configurations and conditions of operation were different. Hence the simultaneous extraction of signals and BGs automatically propagates all sources of uncertainties associated with BGs in the LETA. 


\subsubsection{Treatment of Systematic Uncertainties}

The improvements mentioned in Chapter 3 contributed to make the detector simulation more accurate, which led to a better assignment of the systematic uncertainties that are most of the time derived from the comparison of calibration and simulation data. In the LETA, most systematic errors evaluated from those comparisons have a central value and a spread from which they are propagated into the SigEx procedure through either the method of virtual shifts or by allowing their central values to be constrained by the neutrino data itself.

\section{Systematic Uncertainties as Floating Parameters}

The dominant systematic uncertainties, associated with $T_{\text {eff }}$ and $\beta_{14}$, are integrated in SigEx such that their value can vary (or float) within a pre-determined allowed range. As most systematic errors were determined with calibration sources, the main advantage of allowing them to vary is the verification of their central value with neutrino data: the best value of each systematic is chosen by the data. Because this technique is computationally intensive, only the dominant systematic errors have floating parameters in the extraction. Similar to BGs, floating the dominant systematic errors together with the signals and BGs propagate their effects to the fractions of fitted neutrino events. For example, the energy scale and resolution are allowed to shift to modify the analysis acceptance to low energy BG events near the threshold $T_{\text {eff }}^{0}$. Since a range of scale and resolution are tried by the minimizer simultaneously, these energy systematic errors and their correlations with all observables are automatically propagated into the fractions of fitted neutrino events. 


\subsection{Likelihood Fit}

The statistical separation of the signal and BG classes was performed with an extended log-likelihood function, where the extended part of the fit takes into account the Poisson variation of the total number of events extracted [101]. This section describes the formalism and the specific configuration of the SigEx procedure used with the SNO data.

\subsubsection{Formalism}

The general form of an unbinned extended log-likelihood is:

$$
\log L(\nu, \vec{\theta})=-\nu+\sum_{i=1}^{n} \log \left[\nu f\left(\vec{x}_{i} ; \vec{\theta}\right)\right]
$$

where $n$ is the total number of events in the data, $\nu$ the fitted total number of events, $\vec{x}_{i}$ the observable values for event $i, \vec{\theta}$ the fitted parameters, and $f\left(\vec{x}_{i} ; \vec{\theta}\right)$ the PDF of event $i$ to have observable values $\vec{x}_{i}$, given a model with parameters $\vec{\theta}$.

For practical reasons, a binned negative log-likelihood function was used such that it could be integrated with standard minimizing algorithms. The function, for a single SNO phase, when fitting for $m$ unconstrained CC and ES $T_{\text {eff }}$ bins (indexed with $k$ ), constrained $\mathrm{NC}$ and $\mathrm{BG}$ shapes, using penalty terms for $\mathrm{BG}$ yields, is:

$$
\begin{aligned}
-\log L(\vec{\nu})= & \frac{1}{2} \sum_{j \in\{\mathrm{BG}\}}\left(\frac{\nu_{j}-\left\langle n_{j}\right\rangle}{\sigma_{j}}\right)^{2}+\sum_{j \in\{\mathrm{CC}, \mathrm{ES}\}} \sum_{k=1}^{m} \nu_{j k}+\sum_{j \in\{\mathrm{NC}, \mathrm{BG}\}} \nu_{j} \\
& -\sum_{i=1}^{n} \log \left\{\sum_{j \in\{\mathrm{CC}, \mathrm{ES}\}} \sum_{k=1}^{m} \nu_{j k} f\left(\vec{x}_{i} \mid j, k\right)+\sum_{j \in\{\mathrm{NC}, \mathrm{BG}\}} \nu_{j} f\left(\vec{x}_{i} \mid j\right)\right\},
\end{aligned}
$$


where $\nu_{j}\left(\nu_{j k}\right)$ is the fitted number of events for class $j$ (in the energy bin $k$ ) and $\left\langle n_{j}\right\rangle$ is the expected or measured number of $\mathrm{BG}$ events with uncertainty $\sigma_{j}$. The first term in Equation (5.3) penalizes the BGs. The conditional PDFs, $f\left(\vec{x}_{i} \mid j, k\right)$ and $f\left(\vec{x}_{i} \mid j\right)$, are built from MC events that encompass the full SNO detector response.

The combined nature of the LETA fit introduces constraints on the $\mathrm{D}_{2} \mathrm{O}$ and salt fluxes; the signals $\nu_{j}$ and $\nu_{j k}$ are uniquely determined. The function of Equation (5.3) was modified such that fractions, $\alpha$, relative to the undistorted MC shapes, were fitted instead of absolute number of events. The undistorted shape corresponds to the prediction of the solar standard model (SSM). Therefore the fitted fractions are relative to the number of events, $n$, that would be generated in SNO according to the SSM. For BGs the definition of the SSM is replaced by the expected number of events given by ex situ measurements whenever it is possible. In addition, some systematic uncertainties, $\vec{\xi}$, that are allowed to float with the rest of the parameters are applied to the number of events such that $n \rightarrow n(\vec{\xi})$. Thus the detailed likelihood function is:

$$
\begin{aligned}
-\log L= & \sum_{l \in\left\{\mathrm{D}_{2} \mathrm{O}, \mathrm{Salt}\right\}}\left(\sum_{j \in\{\mathrm{CC}, \mathrm{ES}\}}\left[\left\langle n_{j l}(\vec{\xi})\right\rangle \sum_{k=1}^{m} P(k \mid j, l, \vec{\xi}) \alpha_{j k}\right]\right. \\
& +\sum_{j \in\{\mathrm{NC}, l \mathrm{BG}\}}\left\langle n_{j l}(\vec{\xi})\right\rangle \alpha_{j}+\frac{1}{2} \sum_{j \in\{l \mathrm{BG}\}}\left(\frac{\left\langle n_{j l}(\vec{\xi})\right\rangle-\left\langle n_{j l}(0)\right\rangle}{\sigma_{j l}}\right)^{2} \\
& -\sum_{i=1}^{N} n_{i} \log \left\{\sum_{j \in\{\mathrm{CC}, \mathrm{ES}\}}\right. \\
& {\left[\sum_{k=1}^{m} \delta\left(T_{\mathrm{eff} i} \in k\right)\left\langle n_{j l}(\vec{\xi})\right\rangle \alpha_{j k} f\left(T_{\mathrm{eff} i} \mid j, l, \vec{\xi}\right) f\left(\cos \theta_{\odot_{i}}, \rho_{i}, \beta_{14 i} \mid k, j, l, \vec{\xi}\right)\right] } \\
& \left.\left.+\sum_{j \in\{\mathrm{NC}, l \mathrm{BG}\}}\left\langle n_{j l}(\vec{\xi})\right\rangle \alpha_{j} f\left(T_{\mathrm{eff} i}, \rho_{i}, \beta_{14 i} \mid j, l, \vec{\xi}\right) f\left(\cos \theta_{\odot_{i}} \mid j, l, \vec{\xi}\right)\right\}\right),
\end{aligned}
$$

with variables defined below: 
$\left\langle n_{j l}\right\rangle$ Expected number of events for class $j$ in phase $l$.

$\vec{\xi}$ Systematic uncertainty parameters.

$P(k \mid j, l)$ Probability of event of class type $j$ in phase $l$ to have an energy falling in energy bin $k$.

$\alpha_{j(k)}$ Fitted fraction of events for class $j$ and in energy bin $k$ when applicable.

$\sigma_{j l}$ Constraint on the measured number of events for BG $j$ in phase $l$.

$N$ Number of bins in observable space.

$n_{i}$ Number of events in bin $i$ of observable space.

$\delta\left(T_{\text {eff } i} \in k\right)$ Dirac delta function for energy mapping in bin $k$.

$f(. .$.$) Multidimensional conditional probability density function.$

The fraction of events $\alpha_{j(k)}$ for signal classes $j$ have common parameters for both phases, constraining them by default to the same value. The fraction of events $\alpha_{j}$ for BG classes have independent values in the two phases $l$.

\subsubsection{Configuration}

The complexity of the likelihood function of Equation (5.4) requires many safety checks and a configuration that ensures the neutrino fluxes are extracted without bias. This subsection mentions the data selection, corrections, observable ranges, systematic uncertainties, PDF construction method, and gives the results of ensemble tests performed to verify that the method can extract the neutrino fluxes accurately. 


\section{Data Selection and Corrections}

The neutrino data were selected by a committee of experts who built a run list based on the detector condition and quality of the data taken in various runs during each respective phase of SNO. The selected data were then filtered with an extensive set of instrumental cuts to remove events that are not physics candidates. Then a second set of criteria removed additional events based on a secondary set of observables, such as PMT charges, fraction of PMTs within a given time window, and fiducial volume. The latter are called high level cuts (HLCs). The selection in $T_{\text {eff }}, \beta_{14}, \cos \theta_{\odot}$, and $\rho$ is part of the HLCs. A complete list and description of the cuts applied to the combined

$\mathrm{D}_{2} \mathrm{O}+$ salt data set with $T_{\text {eff }} \geq 3.5 \mathrm{MeV}$ and $r \leq 550 \mathrm{~cm}$ can be found in [83, 114]. The most important ones that apply to the SigEx observables are summarized in Table 5.1. These cuts reduce the amount of data from millions to approximately $100 \mathrm{k}$ events in the LETA.

The MC and real data were corrected to account for known simulation or detector problems [114]. Namely, the MC NC events were corrected for radiative corrections that were not incorporated in the default cross-section model. More importantly, the $z$-coordinates were shifted due to differences in the geometries, and the $\mathrm{D}_{2} \mathrm{O}$ phase energies $T_{\text {eff }}$ were corrected for a known energy response drift using the date of the events. Finally, an energy correction [113] was also applied to account for nonuniformity in the energy response as a function of position in the detector. The MC and data were directly processed by the fit after the selection criteria and corrections were applied. 


\section{Observable Limits and Fit Parameters}

Table 5.1 gives the binning and selection ranges of the four observables. The constrained energy $T_{\text {eff }}^{C}$ (NC and BGs) is finely binned at low energy to accommodate the binned likelihood function with the steep rising slope with decreasing energy of the number of events due to BGs. Otherwise $0.5-\mathrm{MeV}$ bins are defined up to $12 \mathrm{MeV}$ to ensure enough MC statistics are available in each bin of the constructed PDFs. The last bin covers the range between 12 and $20 \mathrm{MeV}$ due to the smallness of the expected number of events above $12 \mathrm{MeV}$. As the table indicates there is only one fit parameter per constrained spectrum for NC and BGs.

The unconstrained energy $T_{\text {eff }}^{U}$ (CC and ES spectra) is binned in a similar manner. The main difference is that the number of fit parameters allows the spectra to be distorted with the independent determination of 14 amplitudes. The variable bin widths were chosen to optimize the CC and ES event statistics in each bin. The ranges and binning of the remaining three observables is also given in Table 5.1. Values of 0 identify observables that discriminate each class via their shape.

The fit parameters thus correspond to the amplitudes of the NC, CC, ES, and BG classes as a function of $T_{\text {eff }}$. This parameterization allows for the total neutrino flux to be extracted from the $\mathrm{NC}$ event fraction, and the effect of the survival probability to be deduced from the distortions of the CC and ES spectral bins with respect to the SSM prediction. In addition, the hypothesis of matter effects in the Earth are investigated by dividing the $\mathrm{CC}$ and ES events into day (D) and night (N) spectra using the time stamp of the events as recorded by the DAQ.

Table 5.2 lists all the fit parameters, separately in terms of event fractions, BGs and systematic uncertainties. The binning in $T_{\text {eff }}$ from Table 5.1 sets the number of 


\begin{tabular}{|c|c|c|c||c|c|}
\hline \hline Observable $i$ & Limits & $N_{\text {bins }}^{i}$ & PDF Range & $N_{\text {fit }}^{i}$ & Fit Range \\
\hline \hline$T_{\text {eff }}^{C}$ & {$[3.5,20]$} & 21 & $\begin{array}{c}0.25[3.5,5] \\
0.5[5,12]\end{array}$ & 1 & \\
(Constrained) & & & $8.0[12,20]$ & & \\
\hline & & & $0.25[3.5,5]$ & & $0.5[3.5,9]$ \\
$T_{\text {eff }}^{U}$ & {$[3.5,20]$} & 21 & $\begin{array}{c}0.5[5,12] \\
8.0[12,20]\end{array}$ & 14 & $\begin{array}{c}1.0[9,10] \\
2.0[10,12] \\
8.0[12,20]\end{array}$ \\
(Unconstrained) & & & & & \\
\hline$\beta_{14}$ & {$[-0.12,0.95]$} & 15 & $0.071 \overline{3}$ & 0 & - \\
\hline $\cos \theta_{\odot}$ & {$[-1,1]$} & 8 & 0.25 & 0 & - \\
\hline$\rho$ & {$[0,0.77025]$} & 5 & 0.15405 & 0 & - \\
\hline \hline
\end{tabular}

Table 5.1: Binning and analysis range of the various observables. The units of $T_{\text {eff }}$ are all MeVs. The value of $N_{\text {bins }}^{i}$ gives the number of bins in the PDFs and the second value $N_{\text {fit }}^{i}$ is the number bins that are allowed to vary in the fit. See text for details.

neutrino event fractions extracted from the data. The day and night time separation effectively doubles the number of CC and ES parameters. The NC flux, BG yields and systematic errors are extracted from the entire data set weighted by the average (A) SNO livetime.

The most important BGs, from the sources explained in Chapter 2, are duplicated in each phase and in each region of the $\rho$ parameter $\left(\mathrm{D}_{2} \mathrm{O}\right.$ or internal, $\mathrm{AV}$, and $\mathrm{H}_{2} \mathrm{O}$ or external). For example the $\mathrm{Bi}$ events are distinct in the $\mathrm{D}_{2} \mathrm{O}$ and salt phases, and have distinct shapes in all observables depending on their origin $\left(\mathrm{D}_{2} \mathrm{O}, \mathrm{H}_{2} \mathrm{O}\right.$ or $\left.A V\right)$, hence 6 constrained amplitudes are extracted in the fit. The $\mathrm{PMT}_{\gamma}^{\beta}$ background was determined analytically from the data [114]; its PDF parameterization can be found in Appendix B. Its scale is allowed to vary in the fit, but four of the five parameters that describe the shape are also defined as systematic parameters. In total there are 17 BG parameters in the fit. 


\begin{tabular}{|l|c||l|l||l|l|}
\hline \hline \multicolumn{2}{|c||}{ Event fractions } & \multicolumn{2}{l||}{ Backgrounds } & \multicolumn{2}{l|}{ Systematic errors } \\
\hline CC-D & 14 & $\mathrm{Bi}$ & 6 & $T_{\text {eff }}$ scale & 3 \\
CC-N & 14 & $\mathrm{Tl}$ & 6 & $T_{\text {eff resolution }}$ & 3 \\
ES-D & 14 & $\mathrm{AV} n$ & 2 & $\beta_{14}$ scale & 3 \\
ES-N & 14 & $\mathrm{PMT}_{\gamma}^{\beta}$ & 2 & $\mathrm{PMT}_{\gamma}^{\beta}$ & 8 \\
NC-A & 1 & Salt $^{24} \mathrm{Na}$ & 1 & & \\
\hline \multicolumn{5}{|c||}{$N_{\text {obs }}=57$} & \multicolumn{3}{|c||}{$N_{\text {bgs }}=17$} & \multicolumn{2}{|c|}{$N_{\text {sys }}=17$} \\
\hline \multicolumn{5}{|c|}{$N_{\text {par }}=91$} \\
\hline
\end{tabular}

Table 5.2: Parameters in the signal extraction procedure. The fraction of events are tagged for day (D), night (N), and average (A). For day-night fits the number of CC and ES observables double, but all other parameters are common and correspond to the average. Typically the BGs have one parameter in each phase plus one parameter per medium: $\mathrm{D}_{2} \mathrm{O}$, $\mathrm{AV}$, and $\mathrm{H}_{2} \mathrm{O}$. Similarly the systematic uncertainties have one parameter per phase, and either one additional correlated parameter or a duplicated parameter to account for the distinct signatures of electrons and neutrons. See Appendix B for details.

The systematic uncertainties that vary in the fit are the energy scale, resolution, $\beta_{14}$ scale, and analytic parameters of the $\mathrm{PMT}_{\gamma}^{\beta}$ background model. The energy scale is divided into phases and has an additional common parameter coming from the calibration source model uncertainties [58]. The energy resolution is also split into phases and a distinction is made for the electron and neutron resolution in the salt phase. The isotropy $\beta_{14}$ is split into electrons and $\mathrm{D}_{2} \mathrm{O}$-phase neutrons, and saltphase neutrons. There is an energy-dependence of $\beta_{14}$ that is also taken into account. Finally the $\mathrm{D}_{2} \mathrm{O}$ - and salt-phase $\mathrm{PMT}_{\gamma}^{\beta}$ background shapes contribute with 8 more parameters, where most of them have stringent constraints [114]. In total there are 17 systematic parameters in the fit. The complete list of parameters in the SigEx fit is given in Table B.1 of Appendix B. 


\section{PDF Factorization}

The expression of the likelihood function in Equation (5.4) implicitly showed the parametrization of the PDFs in the LETA $[80,113]$. In an optimal analysis, the PDFs would be built from multiple 3D histograms with narrow bins filled with high MC statistics to ensure the correlations between all the observables are fully taken into account. The reality is somewhat different, especially for BGs, where the PDFs are limited by the efficiency of the MC generators. Therefore the statistics only allow one to build partially correlated PDFs.

For $\mathrm{CC}$ and $\mathrm{ES}$, the unconstrained nature of the spectra allows for the construction of 3D PDFs in each $T_{\text {eff }}$ bin $k: f\left(\cos \theta_{\odot}, \rho, \beta_{14} \mid k, j, l\right)$. The correlations between $\beta_{14}$, $\rho$, and $\cos \theta_{\odot}$ are therefore fully taken into account in each independent bin of $T_{\text {eff }}$ for each phase $l$. For NC and BGs, the constrained spectra and the expected weak correlation between $\cos \theta_{\odot}$ and other observables allows one to factorize the PDFs: $f\left(T_{\text {eff }}, \rho, \beta_{14} \mid k, j, l\right) \times f\left(\cos \theta_{\odot} \mid k, j, l\right)$. Subsection 5.2.3 shows that the fit results should not be affected by the above factorization of $\mathrm{NC}, \mathrm{CC}, \mathrm{ES}$, and BG PDFs.

\subsubsection{Verification of the Extraction Method}

Several codes can perform signal extraction within the SNO Collaboration $[61,80,81$, $83,113,116]$. The package used in this thesis was implemented recently by Pierre-Luc Drouin [117] at Carleton University to allow more flexibility in the treatment of systematic uncertainties in the binned likelihood fit. The $\mathrm{C}++$ package is called QSigEx-2 for Signal Extraction, second version. The minimizer within is TMinuit

from ROOT [102], the object-oriented version of MINUIT [118] that can handle a large number of parameters. The following demonstrates that the QSigEx-2 code 
is reliable at extracting the event fractions that were pre-determined in fake data ensembles.

\section{Ensembles, Biases and Pulls}

Ensembles were made from the SNO MC events to mimic the real data as much as possible. The events were randomly separated using their simulated unique identification number (GTID). The procedure to make the data sets was to divide all MC data in two, keeping the first half to make the fake data sets and the second half to build the PDFs. The equivalent of 500 SSM samples were generated for CC, ES and NC events. Hence the number of fake data sets was high. One SSM corresponded to approximately 6262 (8755), 780 (1093), and 909 (3369) events for CC, ES, and NC in the $\mathrm{D}_{2} \mathrm{O}$ (salt) phase, respectively, after all analysis cuts were applied. However, when BG classes were included, the maximum number of fake sets was dominated by the limited statistics of the BGs which consequently limited the number of full samples to only 15 [114].

Each of the 15 fake data sets was fitted with the function of Equation (5.4). The bias and pull, defined in Equations (5.5), were calculated for each parameter $\theta$ obtained from the fit:

$$
\begin{aligned}
\text { Bias } & =\frac{N(\theta)-E(\theta)}{E(\theta)} \\
\text { Pull } & =\frac{N(\theta)-E(\theta)}{\sigma(\theta)}
\end{aligned}
$$

where $N(\theta)$ and $E(\theta)$ are the extracted and true input values of $\theta$, and $\sigma(\theta)$ is the fitted statistical uncertainty on $\theta$. The performance of the fitter could then be evaluated 
based on the mean biases and pulls of all fake data sets. The bias is the fractional difference between the fitted and expected values, while the pull is the significance of the shift in terms of the fit uncertainty. After a large number of ensembles are fitted, the distribution of the biases should be centered around zero and the distribution of pulls should be normal $\mathrm{N}(0,1)$, if the extraction method is unbiased. Therefore the biases test the accuracy in recovering the central values and the pulls check both the fitted mean and uncertainty. Although the 15 fake data sets do not constitute a large number it was enough to judge if the fit is stable and returns the expected quantities. Similar studies were also performed on signal-only fits with a large number of fake data sets and ensured the robustness of the SigEx procedure.

Figure 5.2 shows the biases and pulls obtained from the 74 signal and BG parameters. The CC and ES spectra, and NC flux, are all unbiased when the error bars are considered, with the exception of the first low-energy CC-D and ES-D bins, some ES high-energy bins and some BG signals. The pull spreads show that the fit statistical errors are well-behaved, except in some rare cases of BG signals where the constraints influence the evaluation of the uncertainties. Hence the setup of the code, the definition of the observables, and PDF factorization do not pose significant problems and are suited for the extraction of the solar neutrino fluxes. Section 5.3 follows with the result of the extraction from the real LETA data.

\subsection{Results}

This section contains the fit results of the total NC flux as well as the CC and ES spectra for the $T_{\text {eff }}$ bins defined in Table 5.1. The results are given with total uncertainties, after combining the statistical and systematic parts in quadrature. The 


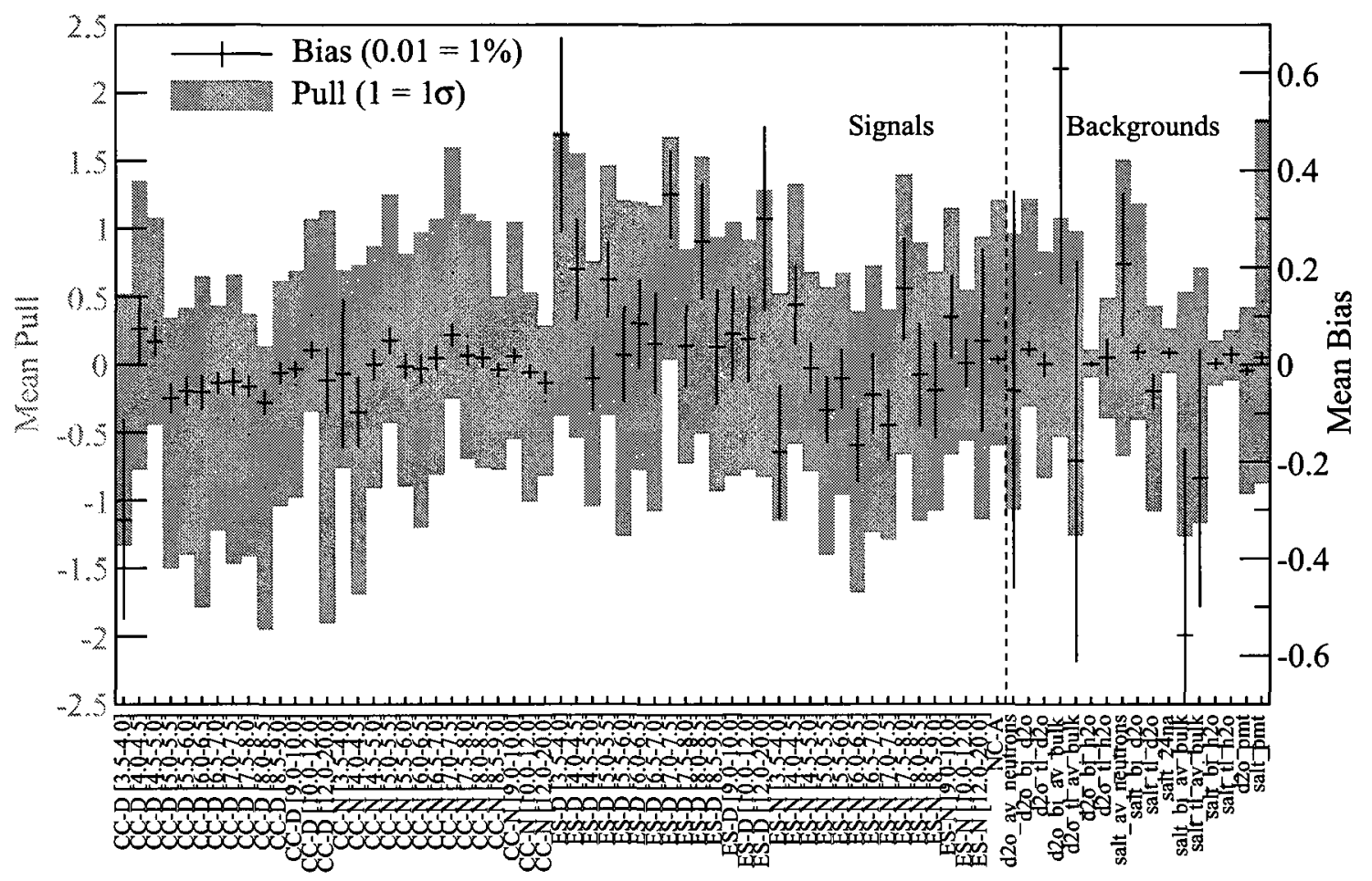

Figure 5.2: Mean biases and pulls of the signal extraction procedure obtained from 15 fake data sets. The signal parameters for day (D), night (N) and average (A) are shown with their range in $T_{\text {eff }}$, in $\mathrm{MeV}$. Parameters without label or energy range are averaged and constrained in $T_{\text {eff }}$ by default. The parameter nomenclature is explained in Appendix B.

statistical uncertainty contains a part of pure statistical variation but also the effect of the BGs and floating systematic uncertainties in the fit. Additional systematic uncertainties, described in Subsection 5.3.3, were evaluated with the shift-and-refit method and added in quadrature to the total uncertainties.

\subsubsection{NC Flux}

The NC flux is insensitive to the solar neutrino flavor, matter and oscillation effects. It was extracted from the whole LETA data set and yields $\alpha_{\mathrm{NC}}=0.91(1.00 \pm 0.04)$ with 
respect to the prediction of the BS05(OP) SSM of $5.69(1.00 \pm 0.16) \times 10^{6} \mathrm{~cm}^{-2} \mathrm{~s}^{-1}$ :

$$
\Phi_{\text {NC }}^{\text {LETA }}=5.18 \pm 0.17 \text { (stat) } \pm 0.12 \text { (syst) } \times 10^{6} \mathrm{~cm}^{-2} \mathrm{~s}^{-1} .
$$

Hence the result is compatible with the SSM prediction. The total uncertainty on the $\mathrm{NC}$ flux is about $4 \%$ which is the most precise determination of the NC flux to date. The combined $\mathrm{D}_{2} \mathrm{O}+$ salt LETA fit has led to a decrease of the uncertainty by $50 \%$ compared to the previous phase-by-phase extraction with higher energy threshold that had uncertainties of the order of $8 \%[27,30]$.

\subsubsection{CC and ES Spectra}

Figure 5.3 shows the day/night CC and ES fractions relative to the SSM prediction. The CC results are shown with total uncertainty bands and the ES results with points and total error bars. The NC flux result is also shown on the right for comparison.

The fractions $\alpha_{\mathrm{CC}}^{k}$ are almost constant past $4.5 \mathrm{MeV}$. The fractions $\alpha_{\mathrm{ES}}^{k}$ do not show this feature but given the large errors they are compatible with a constant value. Assuming the relative rate was constant across all bins, fits were performed to obtain the average fraction for the $\mathrm{CC}$ and ES signals to determine the total fluxes. The CC and ES day (night) average fractions relative to the BS05(OP) SSM were determined to be $0.293 \pm 0.009(0.313 \pm 0.009)$ and $0.557 \pm 0.057(0.472 \pm 0.048)$. The corresponding fluxes are:

$$
\begin{array}{ll}
\Phi_{\mathrm{CC}-\mathrm{D}}^{\mathrm{LETA}}=1.665 \pm 0.051 \pm 0.013 & \Phi_{\mathrm{CC}-\mathrm{N}}^{\mathrm{LETA}}=1.780 \pm 0.046 \pm 0.013 \\
\Phi_{\mathrm{ES}-\mathrm{D}}^{\mathrm{LETA}}=3.170 \pm 0.322 \pm 0.054 & \Phi_{\mathrm{ES}-\mathrm{N}}^{\mathrm{LETA}}=2.685 \pm 0.274 \pm 0.045
\end{array}
$$


where the first component is statistical and the second is systematic, in units of $10^{6} \mathrm{~cm}^{-2} \mathrm{~s}^{-1}$. The day and night results are compatible within the total $1 \sigma$ bounds. Hence there is no evidence of day/night asymmetries in the data.

The unconstrained CC and ES energy spectra are consistent with the previous SNO results for $T_{\text {eff }}>5.5 \mathrm{MeV}$. The CC spectra are determined with an average total uncertainty of $5 \%$. The additional low energy bins are obtained with good precision, except in the first bin, $T_{\text {eff }} \in[3.5,4.0] \mathrm{MeV}$, where BGs dominate over the signals. The large total uncertainty at low energy does not indicate spectral distortion.

Figure 5.4 shows the correlations extracted from the covariance matrix at the best-fit point in parameter space. The correlations are induced by the SigEx fit and are inherent to the statistical method. These correlations are used in the following chapters when calculating correlated errors with respect to the SNO rates.

\subsubsection{Background and Systematic Parameters}

Figure 5.5 shows the fit results for each BG class and systematic uncertainty parameter fitted in SigEx. The parameters all have different scales therefore they are plotted relative to their input values. The bands represent the default values with the constraints, or the penalty term in the likelihood function. The fitted BG fractions are within the original constraints, except the $\mathrm{Bi}$ events from the $\mathrm{AV}$ in the salt phase (salt_bi_av_bulk). It is nevertheless expected to have at least one fitted parameter $\sim 2 \sigma$ away from its expected value.

The fit results of the dominating systematic uncertainties are also shown in Fig-

ure 5.5. All systematic errors are within their predefined range, except a slight shift of the scale of $\beta_{14}$ for electrons and $\mathrm{D}_{2} \mathrm{O}$-phase neutrons (b14Scale0eD2On_u). The size 
SNO Unconstrained CC and ES Day/Night Spectra

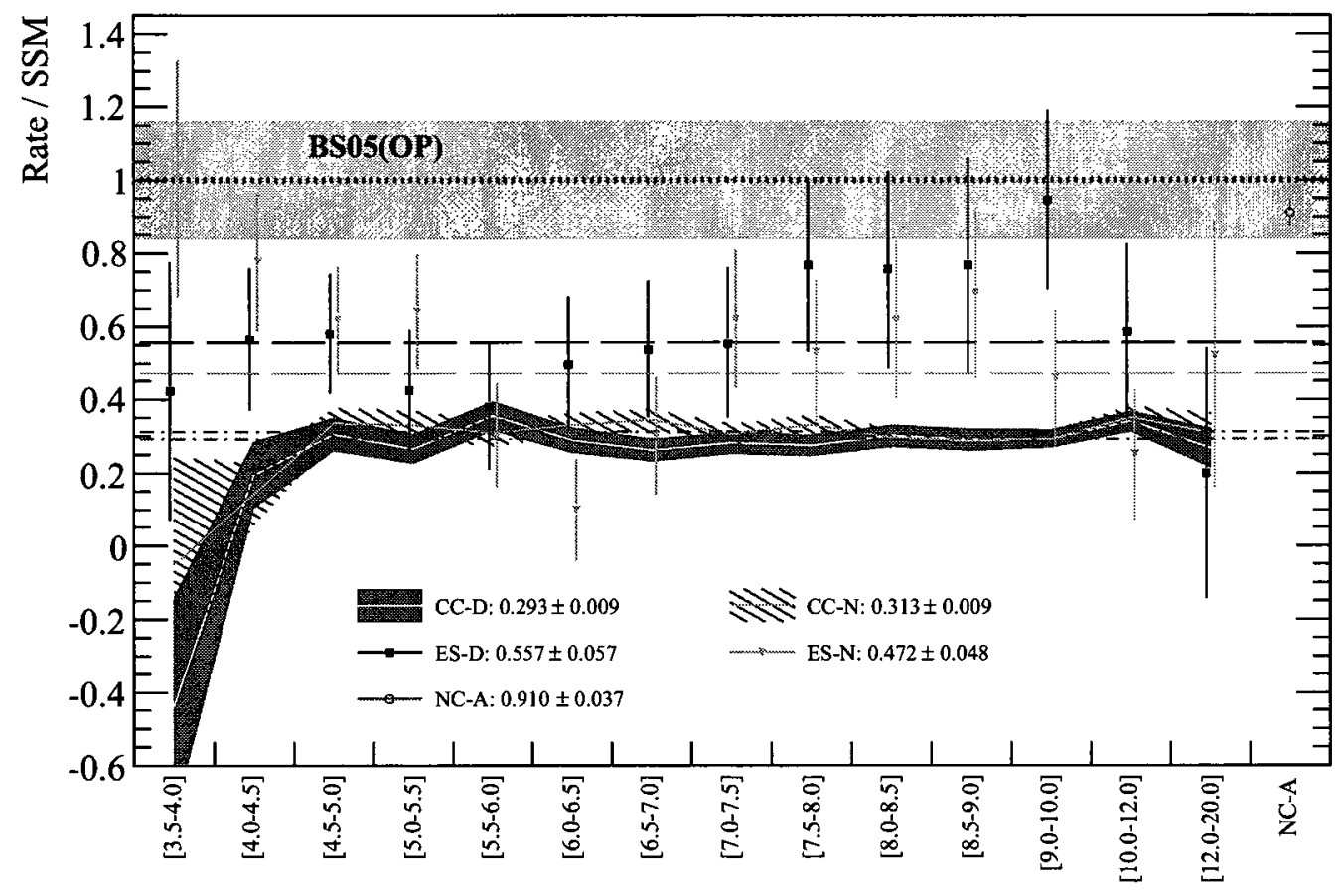

Figure 5.3: (color) Result of the signal extraction procedure. The fraction of events as a function of reconstructed energy $T_{\text {eff }}$ relative to the BS05(OP) prediction are shown with total uncertainties. The orientations are day (D), night $(\mathrm{N})$ and average (A). The NC average rate is indicated on the right for comparison. Also on the right, the average fractions are indicated for each signal; they were obtained using zeroth order fits of the CC and ES spectra.

of the error bars show that the data can indeed constrain the systematic uncertainties and refine the likelihood space near the minimum. The method in QSigEx-2 finds the best value by scanning the systematic parameters until the best-fit is reached. To obtain the effect of these systematic uncertainties on the fluxes, the differences between the fluxes or spectral bins were calculated using the systematic parameters that give a likelihood shift of 0.5 . 


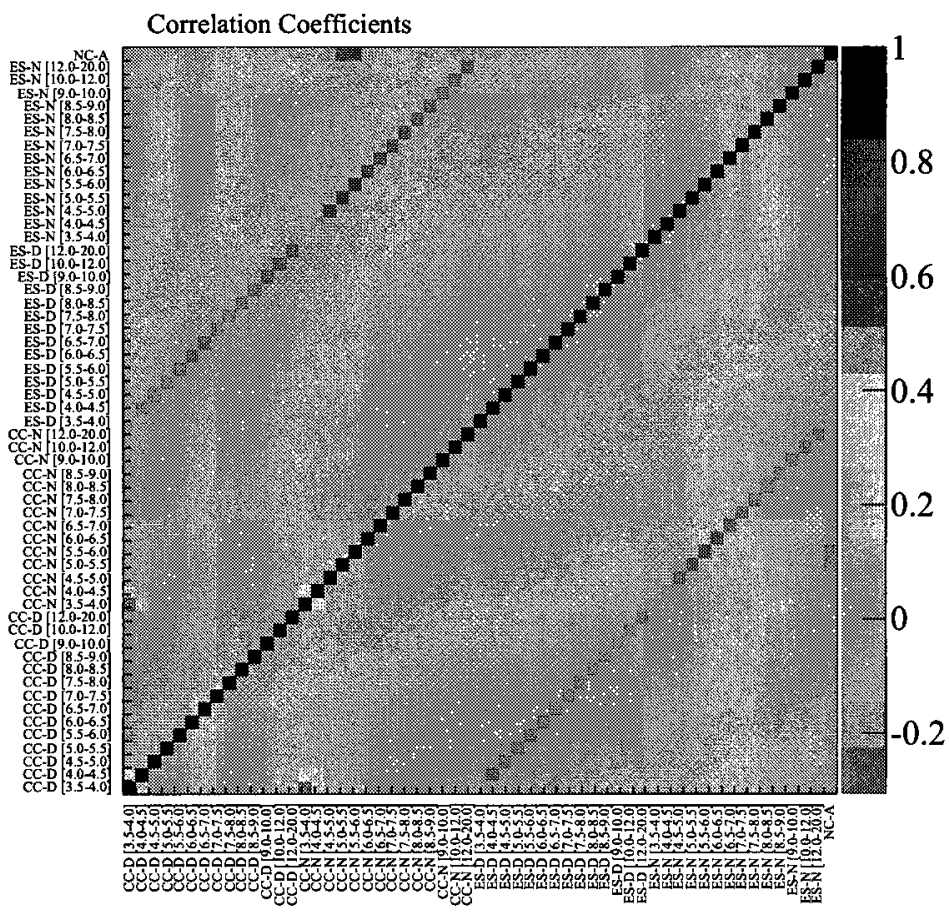

Figure 5.4: (color) Correlation coefficients between the SNO signals. The coefficients were calculated after the systematic parameters were adjusted to their best-fit values.

\section{Additional Systematic Uncertainties}

The effect of other systematic uncertainties were determined with the virtual shift method, also called the shift-and-refit method. While performing the shifts, the floating systematic parameters were fixed to their best-fit values and only the event fractions and BGs were fitted again. In total the effect of 53 systematic uncertainties were evaluated using this technique, including angular resolution, detector axis offsets, vertex coordinate resolution and offsets, model of the ${ }^{24} \mathrm{Na} \mathrm{BG}$, energy-dependent fiducial volume, sacrifice, photo-disintegration rates, neutron capture efficiency, Leslie events, atmospheric $\nu$ 's, a variety of smaller neutron BGs, and finally the linearity of the observables $[80,113,114]$. These systematic effects affect all rates simultaneously 


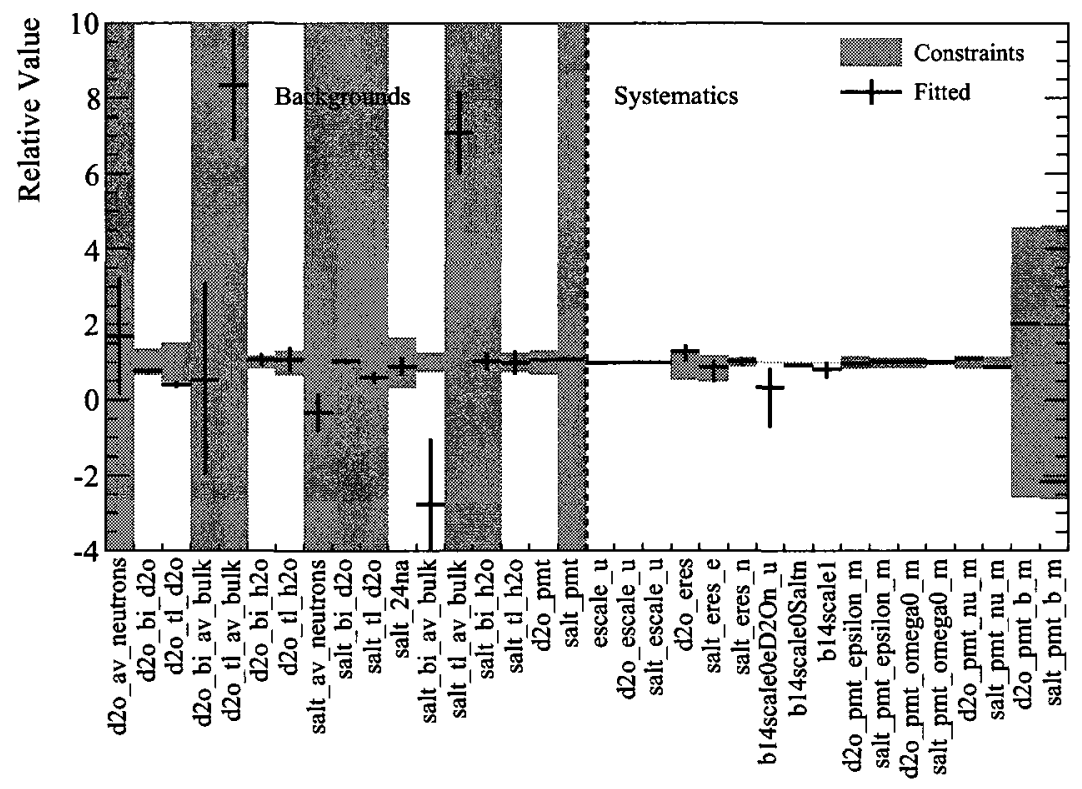

Figure 5.5: Background and systematic uncertainty fitted parameters, relative to their central value. The original constraints are given by the size of the bands and the fit results with the points and errors. There were no constraints if the error bands cover the full range. The error bars of the energy scale and $\beta_{14}$ systematic parameters are very small and cannot be seen on this scale. The parameter nomenclature is explained in Appendix B.

and thus they define an array of spectral derivatives that are all correlated with each other. However, because the floating systematic uncertainties and BGs are extracted simultaneously with the event fractions, these partial derivatives do not dominate the total uncertainties of the results. The common effect of the additional systematic uncertainties on the NC flux is estimated to be of the order of $1 \%$.

\subsubsection{Summary, but not the End}

The low energy threshold analysis is an improvement over previous SNO analyses through the better assessment of the systematic uncertainties, backgrounds, and gain in statistics by performing a combined-phase fit with a lower energy threshold. The 
result of the SigEx fit on the combined $\mathrm{D}_{2} \mathrm{O}+$ salt data set, at an energy threshold of $3.5 \mathrm{MeV}$, is the most precise and interesting result of SNO to date. The results obtained with the QSigex-2 code confirm and will supersede previous published results $[35,80,113]$. One notices that the statistical uncertainties dominate with the simultaneous extraction of BGs. Hopefully the ultimate combination of the LETA with the final NCD phase data [36] will improve the determination of the NC flux and SNO day/night spectra in the near future with the addition of statistics in the fit.

The analysis chain continues with the experimental confirmation of the theory of neutrino oscillation and interpretation of the LETA results in terms of survival probabilities and oscillation parameters. In Chapter 6, the solar survival probability of $\nu_{e}$ 's is extracted. Then, in Chapter 7, the experimental rates are tested against phenomenological models that can predict with high accuracy the value of the survival probability, mainly through the matter-enhanced neutrino oscillation hypothesis. Chapters 6 and 7 are the core of this dissertation and reflect the main contributions of the author to the forthcoming SNO publications $[35,36]$. 


\section{CHAPTER 6}

\section{Extraction of the Survival Probability of Solar Neutrinos}

The next two chapters are about understanding the properties of solar neutrinos and how they oscillate between the core of the Sun and detectors on Earth. This chapter concentrates in particular on the extraction of the survival probability of solar $\nu_{e}$ 's, almost without any model assumption, by comparing measured and expected rates. Results are obtained from the SNO-LETA rates presented in Chapter 5 and from the combination with other solar neutrino experimental data that were mentioned in Chapter 1 . Chapter 7 completes the survival probability analysis with a phenomenological study of the neutrino oscillation parameters in the context of matter-enhanced oscillation. The technical details of the survival probability and oscillation analyses can be found in internal notes [119-127] written by the author.

\subsection{From Neutrino Interactions to Observed Rates}

The survival probability as a function of the neutrino energy, $P_{e e}\left(E_{\nu}\right)$, can be extracted in two equivalent ways. First with an energy-constrained fit, similar to the signal extraction fit, where the MC signal PDFs are continuously distorted with $P_{e e}\left(E_{\nu}\right)$ 
by reweighting the $\mathrm{MC}$ events for which the values of $E_{\nu}$ and $T_{\text {eff }}$ are known. The fit converges at the best set of weights given by the $P_{e e}\left(E_{\nu}\right)$ function, instead of the best CC and ES spectral bins in $T_{\text {eff-space }}[113,128]$. This method has the advantage of fitting a smaller number of parameters and allowing background parameters and systematic uncertainties to adapt to different survival probabilities. However it is computationally extremely intensive because of the large number of events to reweight in the $\mathrm{MC}$ at each iteration of the minimizer.

The second method, preferred in this chapter, consists in finding the best $P_{e e}\left(E_{\nu}\right)$ function that reproduces the fitted, model-independent, unconstrained CC and ES $T_{\text {eff }}$ spectra extracted in Chapter 5. Unconstrained spectra do not have a definite shape in $T_{\text {eff }}$, so this method cannot rely on the MC and must be independent of the SigEx formalism. The analytic convolution method replaces the MC with an analytic rate calculation, where each rate in a $T_{\text {eff }}$ bin is associated with multiple overlapping functions in $E_{\nu}$. Therefore the reweighting from $E_{\nu}$ to $T_{\text {eff }}$ operates on cumulative functions and has the potential of being much faster than reweighting individual MC events as in the SigEx formalism. The convolution converts the rates in neutrino energy to rates in detected energy space. The conversion is achieved by an accurate modeling of the input solar neutrino spectrum, reaction cross-sections and detector response functions. This section focuses on the quantities that are needed to perform that specific conversion.

\subsubsection{Solar Neutrino Spectrum and Survival Probability}

The quantities that characterize solar neutrinos directly, such as the solar neutrino spectrum and survival probability, are expressed as a function of neutrino energy $E_{\nu}$ 
for each neutrino types $\nu_{i}$ defined in Chapter 1:

$$
\begin{aligned}
\nu_{i} & \equiv \text { pp, pep, hep, }{ }^{7} \mathrm{Be},{ }^{8} \mathrm{~B},{ }^{13} \mathrm{~N},{ }^{15} \mathrm{O},{ }^{17} \mathrm{~F} \\
\phi_{\nu_{i}}\left(E_{\nu}\right) & \equiv \text { Solar Neutrino Spectrum } \\
P_{e e}^{\nu_{i}}\left(E_{\nu}\right) & \equiv \text { Survival Probability Function } \\
\phi_{\nu_{i}}^{\mathrm{d}}\left(E_{\nu}\right) & \equiv \text { Observed } \phi_{\nu_{i}}\left(E_{\nu}\right)=\phi_{\nu_{i}}\left(E_{\nu}\right) \times P_{e e}^{\nu_{i}}\left(E_{\nu}\right),
\end{aligned}
$$

for a specific detector (d). Similar to the unconstrained rates that were extracted in the form of ratios with respect to the SSM prediction, the survival probability is extracted from the effective calculation of $\phi_{\nu_{i}}^{\mathrm{d}} / \phi_{\nu_{i}}$. The normalization and shape of the spectra of each flux type, $\Phi_{\nu_{i}}$ and $\phi_{\nu_{i}}$, respectively, are taken from the BS05(OP) SSM. The only exception is the $\nu\left({ }^{8} \mathrm{~B}\right)$ spectrum for which independent and more precise measurements of the shape are available $[129,130]$. The $\nu\left({ }^{8} \mathrm{~B}\right)$ spectrum from [130] is preferred in this thesis and in general in the literature, where its shape and uncertainty are provided in tables [130] with $0.1-\mathrm{MeV}$ bins.

\subsubsection{Interaction Cross-Sections}

The observed spectrum in SNO is different from $\phi_{\nu_{i}}\left(E_{\nu}\right)$ because of the target material cross-section. The SNO reactions are given by cross-section functions providing the probability of the by-products to be emitted with recoil energy $T_{e}$ as a function of both $E_{\nu}$ and $T_{e}$. These functions are shown in Figure 6.1, where the CC and ES differential cross-sections have been integrated over all recoil energies and angular directions.

The cross-section of $\nu$ 's on deuterons, for both $\mathrm{CC}$ and NC, are taken from the 
calculation of Butler-Chen-Kong (BCK) [131]. The BCK calculation includes the effects of radiative corrections but yields uncertainties of the order of $1-1.2 \%[132,133]$ on the absolute $\mathrm{CC}$ and $\mathrm{NC}$ rates due to input parameters such as the axial coupling constant $g_{A}=1.2695 \pm 0.0029$ [2] and the effect of the isovector axial two-body current through $L_{1, A} \sim 5.6 \mathrm{fm}^{3}$ [134]. Similarly, the ES cross-section was calculated by Bahcall [135], including the effects of radiative corrections, and induces an uncertainty of $0.4 \%$ on the ES rate.

The lengthy calculations were performed with small bin sizes and tabulated for the three SNO reactions listed above. The tables were interpolated linearly to give smooth functions as in Figure 6.1. Despite the uncertainties in the cross-section calculations, the $P_{e e}\left(E_{\nu}\right)$ extraction method is mostly insensitive to these uncertainties because of the ratios with respect to the SSM involved in the extraction (see Subsection 6.1.5), in which the latter scaling uncertainties cancel.

\subsubsection{Detector Response}

The CC and ES events in SNO have energies that are correlated with $E_{\nu}$. In both cases, the electron emitted with true kinetic energy $T_{e}$ creates $\check{C}$ light from which the energy $T \equiv T_{\text {eff }}$ is estimated. The probability of an event with true kinetic energy $T_{e}$ to be detected with energy $T$ is given by the normalized response function $R\left(T_{e}, T\right)$. Therefore the response function must be obtained in order to recreate the resolution of the detector.

$\mathrm{MC}$ simulations are ideal to provide the average response function since for each event the true and reconstructed energies are known. The SNO detector response functions were obtained with dedicated MC sets of electrons for $\mathrm{CC}$ and $\mathrm{ES}$, and 
Cross-Sections in SNO

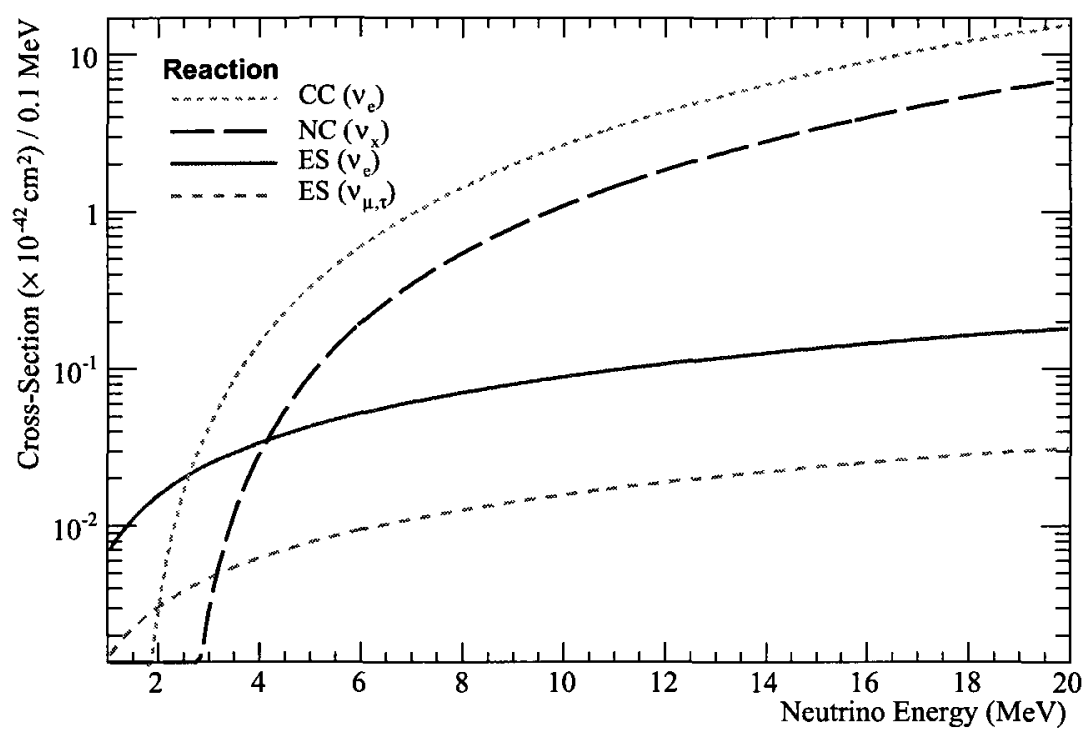

Figure 6.1: Cross-sections of the SNO reactions of Equations (1.17). The sensitivity of the reactions to the neutrino flavor is indicated, where $x=e, \mu, \tau$.

neutrons for NC, independent of those used in the SigEx fit, as explained below.

\section{Response to Electrons}

The MC consisted of pure electron vertices of random positions and isotropic directions within the detector, generated with integer values of kinetic energy $T_{e}$ in $\mathrm{MeV}$. The MC events were selected and corrected, as in Chapter 5, and filled into a 2D histogram, $R_{\mathrm{MC}}^{-}\left(T_{e}, T\right)$. In principle the best response function would be a normalized version of $R_{\mathrm{MC}}^{-}\left(T_{e}, T\right)$ from which the response can be read or interpolated for any given values of $T_{e}$ and $T$. However, because of the discrete values of the generated $T_{e}$ and the need for a fast analytic function, the response function was obtained by projecting $R_{\mathrm{MC}}^{e^{-}}\left(T_{e}, T\right)$ for each $T_{e}$ and by performing a fit to a normal distribution in 
$T$ for each slice in $T_{e}$ :

$$
R^{e^{-}}\left(T_{e}, T \mid T_{e}\right)=\frac{1}{\sigma_{e} \sqrt{2 \pi}} \exp \left[-\frac{\left(T_{e}-T\right)^{2}}{2 \sigma_{e}^{2}}\right]
$$

By repeating the fit for $T_{e} \in[2,24] \mathrm{MeV}$, the function $\sigma_{e} \equiv \sigma_{e}\left(T_{e}\right)$ was also parameterized to form the $2 \mathrm{D}$ analytic response:

$$
\begin{aligned}
R^{e^{-}}\left(T_{e}, T\right) & =\frac{1}{\sigma_{e} \sqrt{2 \pi}} \exp \left[-\frac{\left(T_{e}-(a T+b)\right)^{2}}{2 \sigma_{e}^{2}}\right] \\
\sigma_{e}\left(T_{e}\right) & =r_{0}+r_{1} \sqrt{T_{e}}+r_{2} T_{e}
\end{aligned}
$$

where the energy scale $a$ and offset $b$ are tunable parameters. Figure 6.2 shows the quantities of the SNO electron response function. Figure 6.2a shows the $\mathrm{D}_{2} \mathrm{O}$-phase response of Equation (6.2) at $T_{e}=5 \mathrm{MeV}$ and $12 \mathrm{MeV}$. The width of the distribution increases with $T$, which is explicitly depicted in Figure 6.2b, showing the obtained resolution $\sigma_{e}\left(T_{e}\right)$ of Equation (6.3b) for the three phases of SNO.

\section{Response to Neutrons}

The response to neutrons, $R^{n}(T)$, is a unidimensional function describing the probability of detecting the $\gamma$-ray by-products of the neutron capture from the $\mathrm{NC}$ reaction at a given energy $T$. However, both the weak correlation between the true and observed energy as well as the constrained and integrated nature of the $\mathrm{NC}$ spectrum remove the need for the function $R^{n}(T)$ in this analysis. Nevertheless, Subsection C.1.1 of Appendix C explains how the neutron response function of the SNO detector is parameterized. 


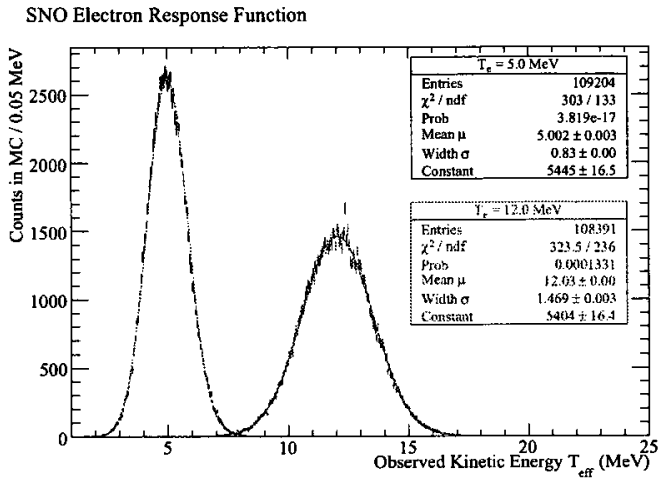

(a) Selected $\mathrm{D}_{2} \mathrm{O}$-phase distributions.

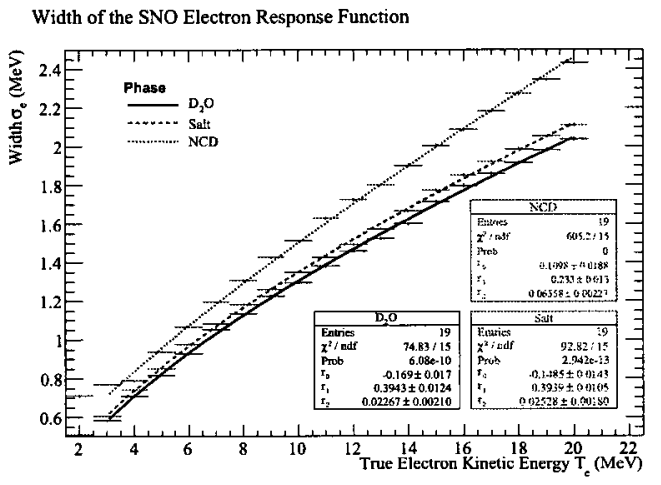

(b) Width $\sigma_{e}\left(T_{e}\right)$.

Figure 6.2: SNO electron response function. (a) The observed energy $T$ corresponds to $T_{\text {eff. The }}$ MC event statistics are given for each fit. (b) The increase of the resolution in the NCD phase is in part due to the shadows created by the counters. The energy estimator for the $\mathrm{D}_{2} \mathrm{O}$ and salt phases is FTK, while it is RSP for the NCD phase.

\subsubsection{Analytic Convolution}

The event rates $N_{T}$ as a function of $T \equiv T_{\text {eff }}$ are calculated by the means of an analytic convolution of the original rates in neutrino energy through the reaction energy thresholds, cross-sections, and detector responses. The arbitrary rates due to electrons, $N_{T}^{e}$, and neutrons, $N^{n}$, were obtained from the solar neutrino spectrum $\phi_{\nu_{i}}\left(E_{\nu}\right)$, the survival probabilities $P_{e e}^{\nu_{i}}\left(E_{\nu}\right)$, the differential $\frac{d \sigma}{d T_{e}}$ and total reaction cross-sections $\sigma\left(E_{\nu}\right)$, the detector response $R$, the particle recoil true $T_{e}$ and observed energy $T$, by numerical summation with small step sizes $d E_{\nu}$ and $d T_{e}$ :

$$
\begin{aligned}
& N_{T}^{e} \propto \int_{0}^{\infty} \phi_{\nu_{i}}\left(E_{\nu}\right) P_{e e}^{\nu_{i}}\left(E_{\nu}\right) d E_{\nu} \int_{0}^{\infty} \frac{d \sigma}{d T_{e}}\left(E_{\nu}, T_{e}\right) d T_{e} \int_{T}^{T+\Delta T} \frac{d R^{e^{-}}}{d T^{\prime}}\left(T_{e}, T^{\prime}\right) d T^{\prime} \\
& N^{n} \propto \int_{0}^{\infty} \phi_{\nu_{i}}\left(E_{\nu}\right) \sigma\left(E_{\nu}\right) d E_{\nu} \int_{T^{0}}^{\infty} \frac{d R^{n}}{d T^{\prime}}\left(T^{\prime}\right) d T^{\prime}=\int_{0}^{\infty} \phi_{\nu_{i}}\left(E_{\nu}\right) \sigma\left(E_{\nu}\right) d E_{\nu}
\end{aligned}
$$


where $\Delta T$ is the bin width in $T$ and $T^{0}$ is the analysis threshold. The arbitrariness in the rates is emphasized by the proportional symbol $(\propto)$, indicating that factors such as the solar spectrum normalization, target volume, livetime, and neutron capture efficiency, needed to obtain the absolute number of events to compare with the number of events in the data, are missing.

The CC and ES spectra in $T$-space are calculated from Equation (6.4a) by selecting the appropriate cross-section. The NC rate is calculated from Equation (6.4b). Note that for ES there is a small component due to the $\nu_{\mu, \tau}$ flux added to Equation (6.4a) when $P_{e e}\left(E_{\nu}\right)<1$, and that $P_{e e}\left(E_{\nu}\right)$ is irrelevant for NC. More generally total rates are obtained from the sum of many components due to the sensitivity to different fluxes $\phi_{\nu_{i}}$.

\subsubsection{Fractional Rates}

Fractional rates, $F_{T}$, are relative to their value calculated with $P_{e e}\left(E_{\nu}\right)=1$, or without neutrino oscillation, as determined from Equations (6.4). Thus they do not depend on correction or scaling factors, flux normalizations, cross-section scales, and detection efficiencies; fractional rates match the definition of fraction of events relative to the SSM prediction, $\alpha$, extracted in Chapter 5 :

$$
\begin{aligned}
& F_{T}^{e}=N_{T}^{e}\left(P_{e e}\left(E_{\nu}\right)\right) / N_{T}^{e}\left(P_{e e}\left(E_{\nu}\right)=1\right) \\
& F^{n}=N^{n} / N^{n}\left(P_{e e}\left(E_{\nu}\right)=1\right) \equiv 1 .
\end{aligned}
$$

For NC, the fractional value is always one by definition since the rate in Equation (6.4b) does not depend on $P_{e e}$. All fractional rates are equal to one when $P_{e e}=1$. 


\section{Comparison with Monte Carlo}

Independent checks were performed to ensure that the analytic calculation of the NC rate and the value predicted by SNOMAN gave similar neutron yields after the appropriate scaling factors were applied [136]. For CC and ES, the resulting shapes of the analytic calculation were also verified. Figure 6.3 shows the CC and ES signal shapes as a function of observed energy obtained from the analytic model compared to the full SNO MC simulation for the three phases of SNO. The shapes, obtained with $P_{e e}\left(E_{\nu}\right)=1$, are normalized to the same area and the colored histograms show the relative differences in each bin of $T_{\text {eff }}$. The largest differences reach $3 \%$ for CC and $5 \%$ for ES. Thus, if absolute rates were used to extract $P_{e e}$, the analytic convolution calculation could introduce a small systematic shape distortion. However, in the following analysis, the fractional rates, relative to the analytic normalization, are systematically compared to the extracted event fractions, relative to the SNO MC, hence removing these systematic offsets.

\subsubsection{Figure of Merit}

In the following, the fractional rates are indexed with $n$, carrying statistical uncertainty $u_{n}$ and total uncertainty $\sigma_{n}$. To each rate is assigned a set of $k$ systematic uncertainties $c_{n k}$. Correlated systematic uncertainties can take different numerical values depending on the rates they affect.

The predicted values of the fractional rates, $F_{n}^{\text {th }}$, from Equations (6.5), may hide dependences on additional parameters, $f_{i}$, which in general are allowed to vary away from their best estimates $f_{i}^{0}$ with constraints $\sigma_{f_{i}}$. For instance, when the parameter $f_{i}$ describe a given systematic uncertainty, the associated systematic errors $c_{n k}$ may 


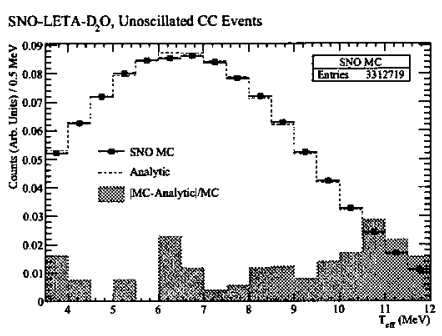

(a) $\mathrm{D}_{2} \mathrm{O}$ CC.

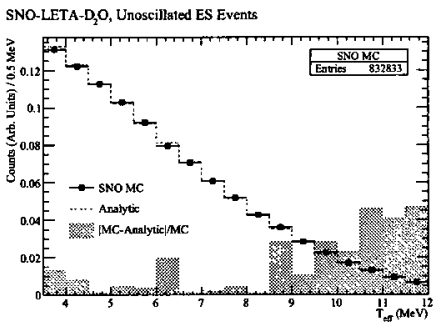

(d) $\mathrm{D}_{2} \mathrm{O}$ ES.

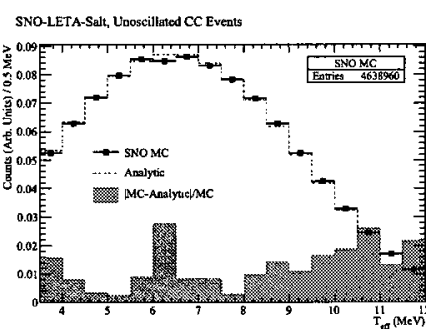

(b) Salt CC.

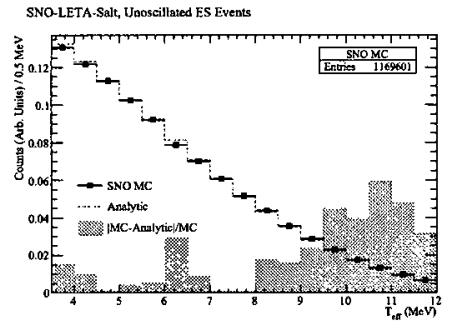

(e) Salt ES.

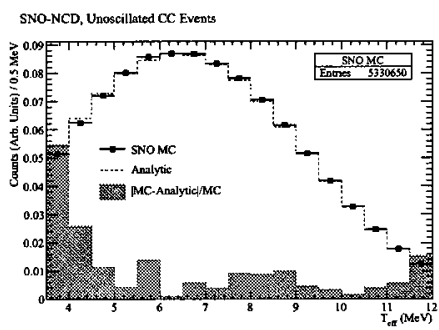

(c) NCD CC.

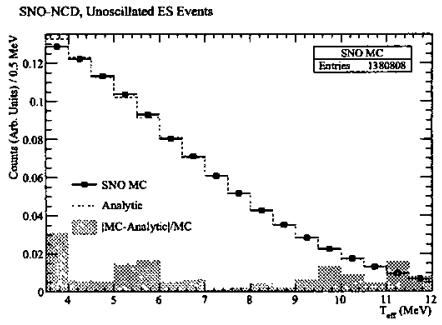

(f) NCD ES.

Figure 6.3: Comparison of the SNO signal shapes obtained from the analytic model and MC simulation, after normalizing both curves to the same area. The horizontal axis is $T_{\text {eff }}$ from 3.5 to $12 \mathrm{MeV}$, with a $0.5 \mathrm{MeV}$ bin width. The histograms give the relative difference in each bin.

also depend on $f_{i}$.

\section{Covariance Method}

As opposed to the multi-dimensional SigEx fit where a likelihood function was used on an event-by-event basis, the analysis of the survival probability is rate-based with $T_{\text {eff }}$ as the only dimension. This is motivated by the fact that the $P_{e e}\left(E_{\nu}\right)$ function is primarily affected by the shape of the rates in $T_{\text {eff }}$, and that the SigEx method has already taken into account the second order effects due to the observables $\beta_{14}, \cos \theta_{\odot}$, and $\rho$ in the unconstrained fit. The rates and their errors follow normal statistics which allow a $\chi^{2}$ figure of merit to be constructed.

The covariance method consists in building the following $\chi^{2}$ function from the 
measured, $F_{n}^{\mathrm{ex}}$, and the predicted, $F_{n}^{\mathrm{th}}\left(f_{i}\right)$, fractional rates:

$$
\chi_{\mathrm{covar}}^{2}=\sum_{n, m}\left(F_{n}^{\mathrm{ex}}-F_{n}^{\mathrm{th}}\left(f_{i}\right)\right)\left[\sigma_{\mathrm{total}}^{-2}\right]_{n m}\left(F_{m}^{\mathrm{ex}}-F_{m}^{\mathrm{th}}\left(f_{i}\right)\right)+\sum_{i}\left(\frac{f_{i}-f_{i}^{0}}{\sigma_{f_{i}}}\right)^{2}
$$

that is minimized with respect to the parameters $f_{i}$ with penalty terms in the second sum. The inverse total error matrix $\left[\sigma_{\text {total }}^{-2}\right]_{n m}$ is composed of the statistical and systematic uncertainties, and includes the statistical correlations between the observables and systematic uncertainties:

$$
\sigma_{\text {total }, n m}^{2}=\rho_{n m}^{\text {stat }} u_{n} u_{m}+\sum_{h, k} \rho_{h k}^{\text {syst }} c_{n h}\left(f_{i}\right) c_{m k}\left(f_{i}\right)
$$

where the correlation coefficients $\rho_{i j} \in[-1,+1]$. The matrix $\sigma_{\text {total,nm }}^{2}$ also depends on the $f_{i}$ even though it is not shown explicitly. Among the solar neutrino experiments studied in this thesis, SNO is the only experiment to necessitate $\rho_{n m}^{\text {stat }}$ because of the correlations inherent to the SigEx procedure (c.f. Section 5.3).

\subsection{Survival Probability Functions}

The accuracy and performance of the analytic survival probability fit relies on the ability to store the fractional rates $F_{n}^{\text {th }}$ as a function of $E_{\nu}$ and $T$ from Equations (6.4) and reweight them for trial values of $P_{e e}\left(E_{\nu}\right)$. At each trial, the rates are simultaneously updated, and the $\chi^{2}$ recalculated. This is repeated until the minimizer finds an accurate minimum. The best-estimate of the function $P_{e e}\left(E_{\nu}\right)$ is extracted at the $\chi_{\min }^{2}$ point.

This section outlines two equivalent parameterizations of the survival probability 
function. Subsection 6.2.1 and 6.2.2 explain the analytic and binned parameterizations, respectively. Subsection 6.2.3 demonstrates they are adequate for the extraction of the survival probability and lastly, Section 6.3 presents the results.

\subsubsection{Analytic Function}

The first parametrization is analytic to ensure the function $P_{e e}\left(E_{\nu}\right)$ is smooth over the whole range of $E_{\nu}$. A general polynomial function is employed:

$$
P_{p}\left(E_{\nu}\right)=p_{0}+p_{1}\left(E_{\nu}-E_{0}\right)+p_{2}\left(E_{\nu}-E_{0}\right)^{2}+p_{3}\left(E_{\nu}-E_{0}\right)^{3},
$$

where $E_{0}$ is the center of the expansion. With such parameterization, $E_{0}$ can be fixed to correspond to the maximum sensitivity of SNO in order to determine the scale of $P_{p}\left(E_{\nu}\right), p_{0}$, with optimal precision. Figure $5.1 \mathrm{~b}$ showed that the LETA CC and ES spectra in $E_{\nu}$-space peaked at around $9.5 \mathrm{MeV}$, hence $E_{0}$ was set to that latter value.

The fitter finds the best-estimates of the parameters, $\hat{p}_{i}$, and evaluates the total uncertainties on each $\hat{p}_{i}, \sigma_{\hat{p}_{i}}$, using the difference $\Delta \chi^{2}=\chi_{\min }^{2}+1$. The nominal curve $P_{p}\left(E_{\nu}\right)$ was built from these best-estimates, and since it is continuous, the $1 \sigma$ uncertainties and covariances $V_{i j}$ form an error band which was obtained from the first order error propagation formula [101]:

$$
\left.\sigma_{P}^{2}\left(E_{\nu}\right) \approx \sum_{i, j} V_{i j}\left[\frac{\partial P_{p}}{\partial p_{i}} \frac{\partial P_{p}}{\partial p_{j}}\right]\right|_{p_{i}=\hat{p}_{i}}
$$

The measurement of the survival probability is therefore $P_{e e}\left(E_{\nu}\right)=P_{p}\left(E_{\nu} \mid \hat{p}_{i}\right) \pm$ $\sigma_{P}\left(E_{\nu} \mid \hat{p}_{i}\right)$ for each $E_{\nu}$. 


\subsubsection{Binned Function}

The second parametrization verifies that the analytic function of Subsection 6.2.1 is not constrained by the specific choice of the polynomial form. Thus $N$ independent parameters, or bins $b_{i}$ of different widths in $E_{\nu}$, are defined to replace the inherent continuous interpolation of the analytic function. Subsection C.1.2 of Appendix C explains how the number of bins was set to $N=5$ with widths given by the six boundaries:

$$
P_{b}\left(E_{\nu}\right)=\{3,6,8,10,12,16\} .
$$

The weights of the bins in the function $P_{b}\left(E_{\nu}\right)$ are labeled $b_{i}$. The errors on the $\hat{b}_{i}$ are obtained by the fitter directly in $E_{\nu}$-space. The measurement of the survival probability is therefore $P_{e e}\left(E_{\nu}\right)=P_{b}\left(E_{\nu} \mid \hat{b}_{i}\right) \pm \sigma_{\hat{b}_{i}}$ for each $E_{\nu}$.

\subsubsection{Verification of the Extraction Method}

A verification procedure similar to that presented in Subsection 5.2.3 was performed to test the two parameterizations of the survival probability. A true function, $P_{e e}^{\text {true }}\left(E_{\nu}\right)$, was employed with the acceptance-rejection method [101] to select CC, ES, and NC events from the SNO MC. Backgrounds and systematic parameters were not necessary for this verification hence 250 fake data sets of CC, ES, and NC events were fabricated. The relative fractions $F_{n}^{\text {ex }}$ from each set were extracted with the SigEx fit and were then fed to the survival probability fitter. The uncertainties gathered from each fit include the pure statistical uncertainties $u_{n}$ and the correlation coefficients $\rho_{n m}^{\text {stat }}$ between the CC, ES, and NC signals.

A true polynomial base function $\bar{P}_{e e}^{\text {true }}\left(E_{\nu}\right)$ was built with parameters $p_{0}=0.352$, 


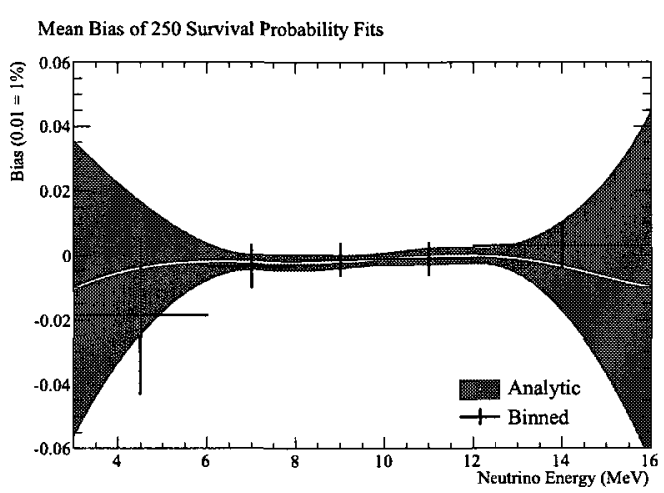

(a) Mean Bias.

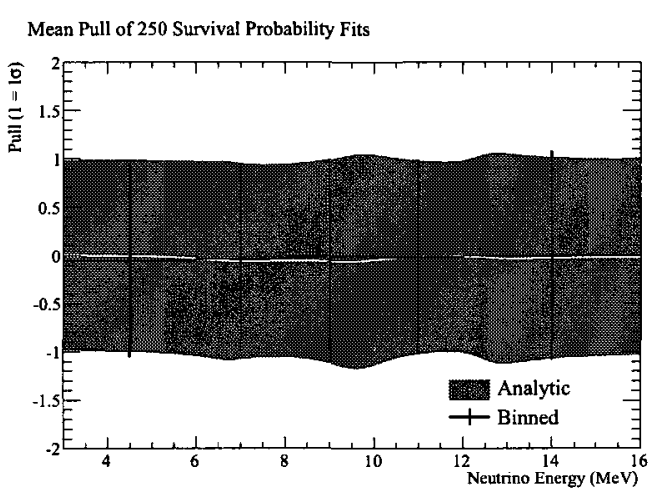

(b) Mean Pull.

Figure 6.4: Mean bias and pull of survival probability fits performed from the results of the unconstrained SigEx fits of 250 ensemble data sets.

$p_{1}=-7.23 \times 10^{-3} \mathrm{MeV}^{-1}, p_{2}=1.13 \times 10^{-3} \mathrm{MeV}^{-2}$, and $p_{3}=-6.73 \times 10^{-5} \mathrm{MeV}^{-3}$, corresponding to a typical region in the oscillation parameter space, called the LMA region (c.f. Chapter 7 ), preferred by previous SNO analyses $[27,30]$. The parameters were varied by small random amounts to obtain a variety of curves, around $\vec{P}_{e e}^{\text {true }}\left(E_{\nu}\right)$, with slightly different shapes. Thus the 250 fake data sets were created with 250 different $P_{e e}^{\text {true }}\left(E_{\nu}\right)$ curves.

Figure 6.4 shows the mean bias and pull, defined in Equations (5.5), obtained after fitting the survival probability of the 250 unconstrained spectra with both analytic and binned parameterizations. Figure $6.4 \mathrm{a}$ shows that the mean biases are consistent with zero for all $E_{\nu}$. Figure $6.4 \mathrm{~b}$ shows that the mean pulls have width of $1 \sigma$, meaning the statistical errors in the fractions $F_{n}^{\mathrm{ex}}$ have propagated as expected into both the band of the analytic curve and the errors of the binned weights. Hence both the analytic and binned functions are well suited to extract $P_{e e}\left(E_{\nu}\right)$ from the SigEx unconstrained results of Chapter 5 . 


\section{Sensitivity to High Orders in $E_{\nu}$}

One interesting question to address with the survival probability fit is whether the order of the true function is lost in the convolution with the cross-section and detector resolution effects. The above function $\bar{P}_{e e}^{\text {true }}\left(E_{\nu}\right)$ was used to reweight the 250 fake data sets to test the sensitivity of the fit to the true third order polynomial nature of a unique survival probability function. This was done with the analytic parameterization only; the order of the function in Equation (6.8) was progressively reduced by setting $p_{3}, p_{2}$, and $p_{1}$ to zero, and the fits repeated.

Figure 6.5 shows the biases obtained from the fits, in the full and most-sensitive range in $E_{\nu}$. Figure 6.5a confirms that zeroth and first order functions are not suitable for the extraction of a true third order function, where biases of the order of $25 \%$ and $10 \%$, respectively, are observed. The second order function departs from the true curve in the range $E_{\nu} \in[3,6] \mathrm{MeV}$ and otherwise performs with good accuracy showing biases within $5 \%$.

Figure $6.5 \mathrm{~b}$ shows that, in the range $E_{\nu} \in[6,13] \mathrm{MeV}$ of best sensitivity for SNO, the second and third order functions are both suitable. The vertical axis is limited to $\pm 3 \%$, which corresponds to the inherent statistical fluctuation in the biases due to the small number of events in each fake data set. In that limit and within the most sensitive region, even the first order function would be giving a reasonable answer. Hence, in the expected best-fit region given by previous oscillation analyses, the test shows that at least setting $p_{3}=0$ in Equation (6.8) would not impact the survival probability fit by more than the expected total uncertainties of the SNO unconstrained spectra. However, the rest of the survival probability analysis presented in this chapter assumes a third order polynomial function in all cases since biases are consistent with 


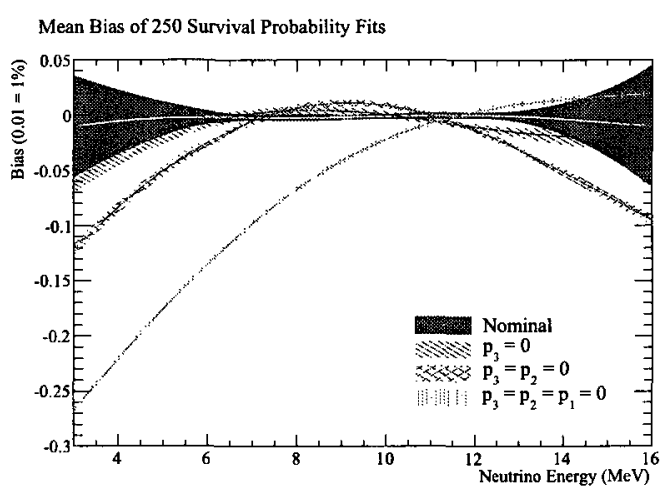

(a) Whole range in $E_{\nu}$.

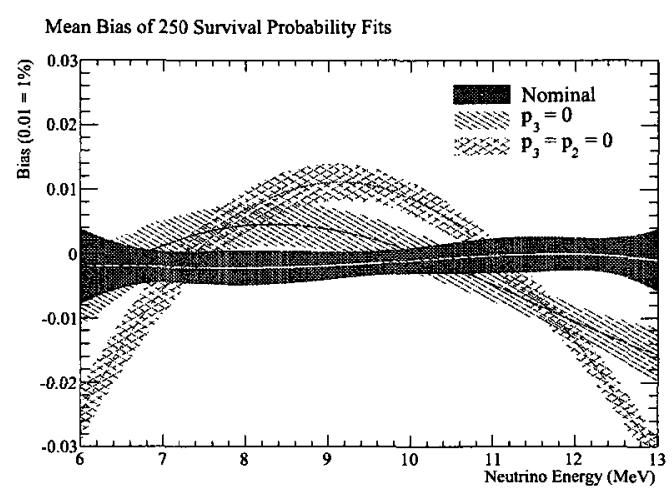

(b) Most sensitive range in $E_{\nu}$.

Figure 6.5: Mean bias of analytic survival probability fits for different orders performed from the results of the unconstrained SigEx fits of 250 ensemble data sets. The nominal function corresponds to the formula of Equation (6.8). The zeroth, first, and second order functions correspond to $p_{3}=p_{2}=p_{1}=0, p_{3}=p_{2}=0$, and $p_{3}=0$, respectively.

zero for all $E_{\nu}$ values.

\subsection{Results}

This section contains the results of the day/night survival probability fits for SNO based on the results of Chapter 5. Then the contributions of other solar neutrino experiments are incorporated to the SNO analysis to obtain a combined and global measurement of the survival and transition probability functions.

\subsubsection{Results from SNO}

The 57 signal parameters resulting from the LETA are the average NC flux and the 14 spectral day/night bins for the CC and ES reactions. They are the inputs $F_{n}^{\text {ex }}$ to the fit. The statistical uncertainties were extracted from the diagonal elements of the SigEx covariance matrix such that they were symmetric. The statistical correlations 
$\rho^{\text {stat }}$ were taken from the off-diagonal elements of the same matrix. The 53 systematic uncertainties $c_{n k}$ that are specific to SNO-LETA were assumed to be fully-correlated in all rates, without correlations to other systematic uncertainties. The uncertainty on the shape of the $\nu\left({ }^{8} \mathrm{~B}\right)$ spectrum [130] was added to the list of systematic uncertainties and treated in the same way.

The day/night nature of the SigEx fit allowed two survival probability functions to be extracted simultaneously, one for day-time and the other for night-time. Additional $f_{i}$ parameters include the flux normalizations $f_{8_{\mathrm{B}}}$ and $f_{\text {hep }}$ and the scale of the $\nu\left({ }^{8} \mathrm{~B}\right)$ spectral shape uncertainty, $s_{8_{\mathrm{B}}}$. The scale $f_{8_{\mathrm{B}}}$ is entirely constrained by the $\mathrm{NC}$ measurement thus there was no penalty term associated with it in the fit. The scale $f_{\text {hep }}$, since it is indistinguishable from $f_{8_{\mathrm{B}}}$, was constrained to the BS05(OP) SSM value with its model uncertainty of $16 \%$ and the hep survival probability was assumed to be degenerate, $P_{e e}^{\mathrm{hep}}\left(E_{\nu}\right)=P_{e e}^{8 \mathrm{~B}}\left(E_{\nu}\right)$. The hep contribution to the total rate is $\sim 10^{-3}$ so it was included for completeness. Finally the scale $s_{8_{\mathrm{B}}}$ was free to vary independently from $f_{\mathrm{B}_{\mathrm{B}}}$.

\section{The Functions}

Figure 6.6 shows the resulting survival probabilities obtained from both the analytic and binned parameterizations. Although the analytic functions do not seem to match with the bins at their centers, there is always reasonable agreement considering the error bars of the bins as well as the intrinsic nature of both fits. As explained with their definitions in Section 6.2, the analytic and binned functions are differentiated by their continuity as a function of $E_{\nu}$. Since the analytic function was designed to be precise near $E_{0}=9.5 \mathrm{MeV}$, the errors grow rapidly as $E_{\nu}$ departs from $E_{0}$. The 
binned function however is more general, but its bins are free to vary independently from each other, which induces large bin-to-bin correlations that are not taken into account when drawing the error bars. The agreement was better for the day fits than for the night fits, partly due to the first night bin that is off-scale. The analytic curves pass between the oscillating bins otherwise, as seen in Figure 6.6b.

The binned fit has more flexibility, then less degrees of freedom, and gave a smaller value of $\chi_{\min }^{2}=56.05$ when compared to 63.67 obtained with the analytic fit. This difference was caused by the direct correlations of the first weight $b_{0} \in[3,6] \mathrm{MeV}$ with the low- $T_{\text {eff }}$ CC and ES bins that were strongly affected by BGs in SigEx.

The fitted $f_{8_{\mathrm{B}}}$ and $f_{\text {hep }}$ in each fit were consistent with the NC flux scale and SSM uncertainties. The scale $s_{\mathrm{B}}$ had no significant effect in the fits with a final value of $1.0 \pm 0.9$ in both fits. Therefore the data could not constrain that systematic effect that had very little impact on the fit results.

Tables 6.1 and 6.2 provide the numerical values of the parameters with their correlations. As expected the $p_{0}$ parameter in Table 6.1 has values that are inconsistent with one; oppositely, the $p_{1}, p_{2}$, and $p_{3}$ parameters were found to be consistent with zero in both day and night cases. Based on the polynomial fit one cannot claim the observation of spectral distortions. From Table 6.2, the binned fit parameters $b_{0}$ are the only ones with large errors. The uncertainties were barely asymmetric, thus they were symmetrized by picking the largest of the positive and negative errors. Although the correlation coefficients are sizable, they were checked to be multinormal for each pair of parameters. 


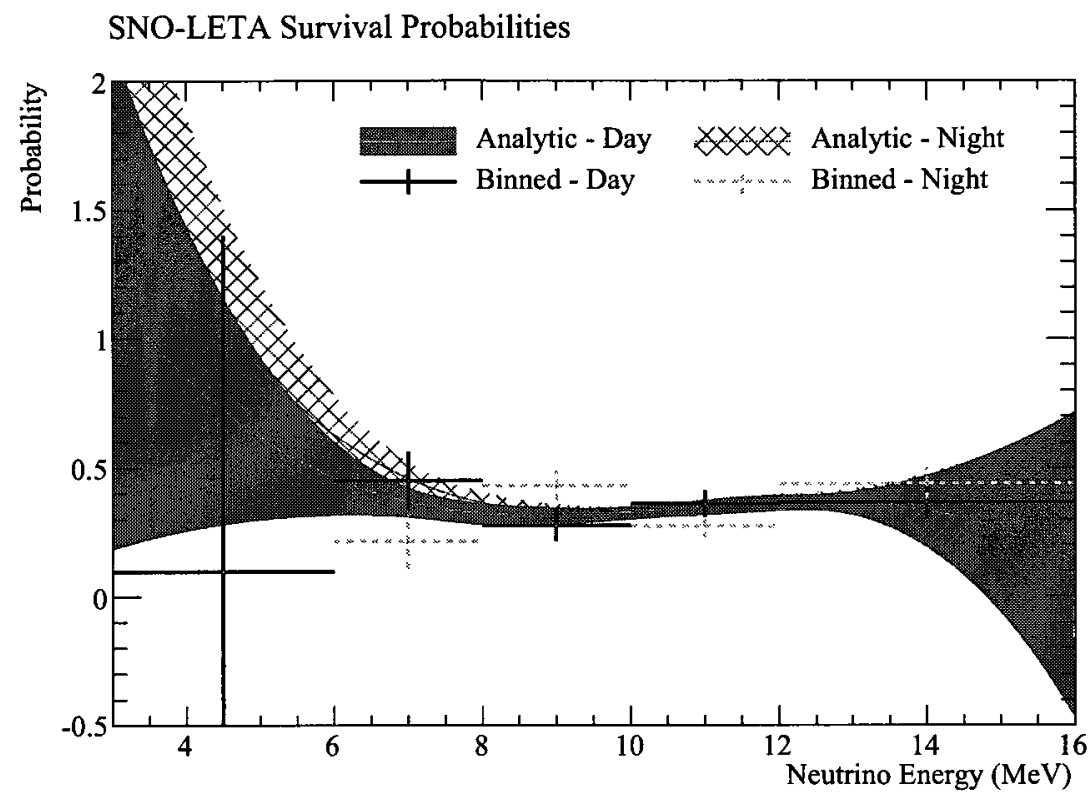

(a) Whole range in $E_{\nu}$.

SNO-LETA Survival Probabilities

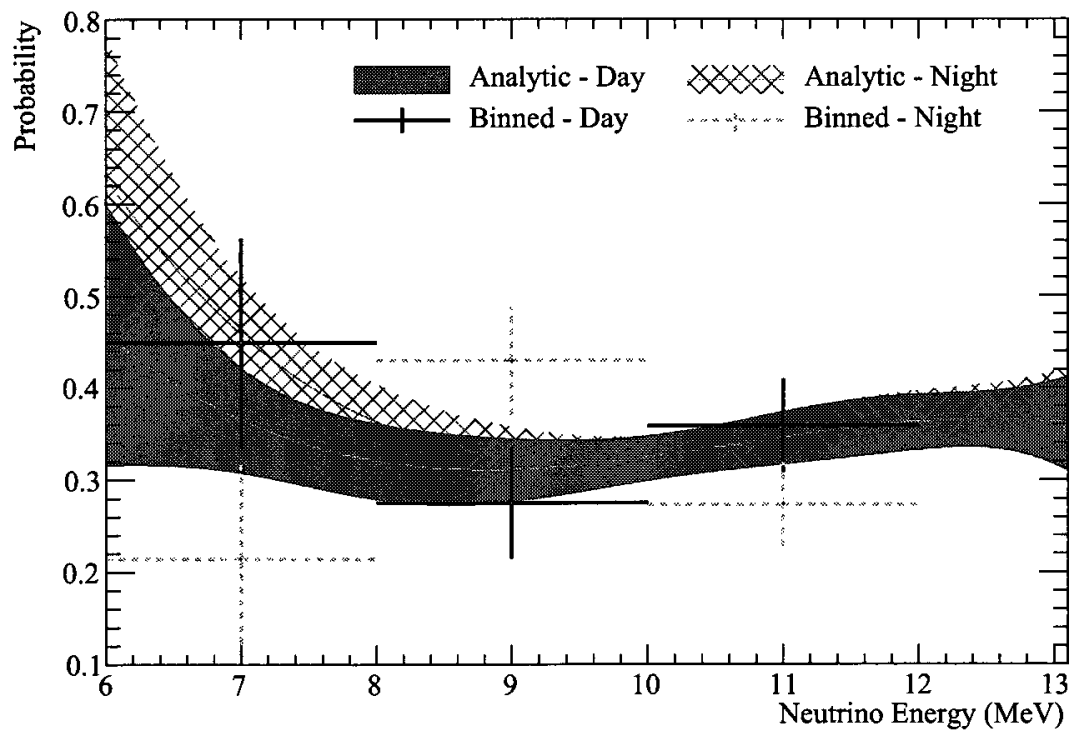

(b) Most sensitive range in $E_{\nu}$.

Figure 6.6: Survival probability functions extracted from the SNO-LETA day/night combined fit of the $\mathrm{D}_{2} \mathrm{O}$ and salt phases. The analytic (continuous) and binned (bars) parameterizations are both shown for completeness. 


\begin{tabular}{|c|c|c|c|c|c|c|c|c|c|c|}
\hline & \multicolumn{2}{|c|}{ Flux Scales } & \multicolumn{4}{|c|}{$\overrightarrow{\text { Day }}$} & \multicolumn{4}{|c|}{ Night } \\
\hline & $f_{8 \mathrm{~B}}$ & $f_{\text {hep }}$ & $p_{0}$ & $p_{1}\left(\mathrm{MeV}^{-1}\right)$ & $p_{2}\left(\mathrm{MeV}^{-2}\right)$ & $p_{3}\left(\mathrm{MeV}^{-3}\right)$ & $\overrightarrow{p_{0}}$ & $p_{1}\left(\mathrm{MeV}^{-1}\right)$ & $p_{2}\left(\mathrm{MeV}^{-2}\right)$ & $p_{3}\left(\mathrm{MeV}^{-3}\right)$ \\
\hline Fit & $0.918 \pm 0.035$ & $0.994 \pm 0.155$ & $0.315 \pm 0.028$ & $0.013 \pm 0.024$ & $0.008 \pm 0.007$ & $-0.002 \pm 0.003$ & $0.321 \pm 0.027$ & $0.001 \pm 0.023$ & $0.015 \pm 0.007$ & $-0.003 \pm 0.003$ \\
\hline $\begin{array}{l}f_{6 \mathrm{~B}} \\
f_{\text {hcp }}\end{array}$ & $\begin{array}{l}+1.000 \\
-0.014\end{array}$ & $\begin{array}{l}-0.014 \\
+1.000\end{array}$ & $\begin{array}{l}+0.003 \\
-0.595\end{array}$ & $\begin{array}{l}+0.023 \\
+0.151\end{array}$ & $\begin{array}{l}-0.001 \\
-0.084\end{array}$ & $\begin{array}{l}-0.027 \\
+0.010\end{array}$ & $\begin{array}{r}-0.003 \\
+0.614\end{array}$ & $\begin{array}{r}+0.027 \\
+0.190\end{array}$ & $\begin{array}{l}+0.001 \\
-0.143\end{array}$ & $\begin{array}{l}-0.031 \\
+0.024\end{array}$ \\
\hline $\begin{array}{l}p_{0} \\
p_{1} \\
p_{2} \\
p_{3}\end{array}$ & $\begin{array}{l}+0.003 \\
+0.023 \\
-0.001 \\
-0.027\end{array}$ & $\begin{array}{l}-0.595 \\
+0.151 \\
-0.084 \\
+0.010\end{array}$ & $\begin{array}{l}+1.000 \\
-0.457 \\
-0.607 \\
+0.456\end{array}$ & $\begin{array}{r}-0.457 \\
+1.000 \\
+0.391 \\
-0.884\end{array}$ & $\begin{array}{l}-0.607 \\
+0.391 \\
+1.000 \\
-0.667\end{array}$ & $\begin{array}{l}+0.456 \\
-0.884 \\
-0.667 \\
+1.000\end{array}$ & $\begin{array}{l}-0.470 \\
+0.145 \\
+0.046 \\
-0.070\end{array}$ & $\begin{array}{l}-0.164 \\
+0.129 \\
+0.020 \\
-0.077\end{array}$ & $\begin{array}{l}+0.024 \\
-0.014 \\
+0.211 \\
-0.107\end{array}$ & $\begin{array}{l}+0.043 \\
-0.062 \\
-0.133 \\
+0.134\end{array}$ \\
\hline $\begin{array}{l}p_{0} \\
p_{1} \\
p_{2} \\
p_{3}\end{array}$ & $\begin{array}{r}-0.003 \\
+0.027 \\
+0.001 \\
-0.031\end{array}$ & $\begin{array}{r}+0.614 \\
+0.190 \\
-0.143 \\
+0.024\end{array}$ & $\begin{array}{l}-0.470 \\
-0.164 \\
+0.024 \\
+0.043\end{array}$ & $\begin{array}{r}+0.145 \\
+0.129 \\
-0.014 \\
-0.062\end{array}$ & $\begin{array}{r}+0.046 \\
+0.020 \\
+0.211 \\
-0.133\end{array}$ & $\begin{array}{l}-0.070 \\
-0.077 \\
-0.107 \\
+0.134\end{array}$ & $\begin{array}{r}+1.000 \\
+0.483 \\
+0.539 \\
-0.454\end{array}$ & $\begin{array}{r}+0.483 \\
+1.000 \\
+0.369 \\
-0.853\end{array}$ & $\begin{array}{r}+0.539 \\
+0.369 \\
+1.000 \\
-0.704\end{array}$ & $\begin{array}{l}-0.454 \\
-0.853 \\
-0.704 \\
+1.000\end{array}$ \\
\hline
\end{tabular}

Table 6.1: Analytic survival probability parameters extracted from the SNO-LETA day/night analysis. The best-fit point gave $\chi^{2} / \mathrm{ndf}=63.67 /(57-10)=1.35$. The correlation coefficients are given in the lower part of the table.

\begin{tabular}{|c|c|c|c|c|c|c|c|c|c|c|c|c|}
\hline & \multicolumn{2}{|c|}{ Flux Scales } & \multicolumn{5}{|c|}{ Day } & \multicolumn{5}{|c|}{ Night } \\
\hline & $f_{s_{B}}$ & $f_{\text {hep }}$ & $b_{0}$ & $b_{1}$ & $b_{2}$ & $b_{3}$ & $b_{4}$ & $b_{0}$ & $b_{1}$ & $b_{2}$ & $b_{3}$ & $b_{4}$ \\
\hline Fit & $0.919 \pm 0.035$ & $0.987 \pm 0.155$ & $0.095 \pm 1.294$ & $0.449 \pm 0.114$ & $0.275 \pm 0.060$ & $0.359 \pm 0.051$ & $0.366 \pm 0.064$ & $2.493 \pm 0.784$ & $0.214 \pm 0.105$ & $0.429 \pm 0.058$ & $0.273 \pm 0.046$ & $0.439 \pm 0.062$ \\
\hline $\begin{array}{l}f_{6_{B}} \\
f_{\text {hop }}\end{array}$ & $\begin{array}{l}+1.000 \\
-0.014\end{array}$ & $\begin{array}{r}-0.014 \\
+1.000\end{array}$ & $\begin{array}{l}-0.002 \\
-0.023\end{array}$ & $\begin{array}{l}+0.006 \\
-0.307\end{array}$ & $\begin{array}{l}-0.011 \\
-0.295\end{array}$ & $\begin{array}{l}+0.022 \\
-0.251\end{array}$ & $\begin{array}{l}-0.033 \\
-0.163\end{array}$ & $\begin{array}{l}-0.001 \\
-0.154\end{array}$ & $\begin{array}{l}+0.007 \\
-0.251\end{array}$ & $\begin{array}{l}-0.011 \\
-0.395\end{array}$ & $\begin{array}{l}+0.025 \\
-0.214\end{array}$ & $\begin{array}{l}-0.036 \\
-0.216\end{array}$ \\
\hline $\begin{array}{l}b_{0} \\
b_{1} \\
b_{2} \\
b_{3} \\
b_{4}\end{array}$ & $\begin{array}{l}-0.002 \\
+0.006 \\
-0.011 \\
+0.022 \\
-0.033\end{array}$ & $\begin{array}{l}-0.023 \\
-0.307 \\
-0.295 \\
-0.251 \\
-0.163\end{array}$ & $\begin{array}{l}+1.000 \\
-0.620 \\
+0.356 \\
-0.214 \\
+0.143\end{array}$ & $\begin{array}{l}-0.620 \\
+1.000 \\
-0.548 \\
+0.496 \\
-0.230\end{array}$ & $\begin{array}{l}+0.356 \\
-0.548 \\
+1.000 \\
-0.630 \\
+0.505\end{array}$ & $\begin{array}{l}-0.214 \\
+0.496 \\
-0.630 \\
+1.000 \\
-0.632\end{array}$ & $\begin{array}{r}+0.143 \\
-0.230 \\
+0.505 \\
-0.632 \\
+1.000\end{array}$ & $\begin{array}{l}+0.190 \\
+0.014 \\
+0.082 \\
+0.016 \\
+0.077\end{array}$ & $\begin{array}{l}-0.050 \\
+0.171 \\
+0.070 \\
+0.070 \\
-0.025\end{array}$ & $\begin{array}{l}+0.023 \\
+0.121 \\
+0.158 \\
+0.085 \\
+0.136\end{array}$ & $\begin{array}{l}-0.008 \\
+0.076 \\
+0.047 \\
+0.105 \\
-0.035\end{array}$ & $\begin{array}{l}+0.036 \\
-0.004 \\
+0.135 \\
-0.005 \\
+0.291\end{array}$ \\
\hline $\begin{array}{l}b_{0} \\
b_{1} \\
b_{2} \\
b_{3} \\
b_{4}\end{array}$ & $\begin{array}{l}-0.001 \\
+0.007 \\
-0.011 \\
+0.025 \\
-0.036\end{array}$ & $\begin{array}{l}-0.154 \\
-0.251 \\
-0.395 \\
-0.214 \\
-0.216\end{array}$ & $\begin{array}{l}+0.190 \\
-0.050 \\
+0.023 \\
-0.008 \\
+0.036\end{array}$ & $\begin{array}{l}+0.014 \\
+0.171 \\
+0.121 \\
+0.076 \\
-0.004\end{array}$ & $\begin{array}{l}+0.082 \\
+0.070 \\
+0.158 \\
+0.047 \\
+0.135\end{array}$ & $\begin{array}{l}+0.016 \\
+0.070 \\
+0.085 \\
+0.105 \\
-0.005\end{array}$ & $\begin{array}{l}+0.077 \\
-0.025 \\
+0.136 \\
-0.035 \\
+0.291\end{array}$ & $\begin{array}{l}+1.000 \\
-0.540 \\
+0.380 \\
-0.164 \\
+0.179\end{array}$ & $\begin{array}{l}-0.540 \\
+1.000 \\
-0.526 \\
+0.488 \\
-0.239\end{array}$ & $\begin{array}{l}+0.380 \\
-0.526 \\
+1.000 \\
-0.593 \\
+0.521\end{array}$ & $\begin{array}{l}-0.164 \\
+0.488 \\
-0.593 \\
+1.000 \\
-0.626\end{array}$ & $\begin{array}{l}+0.179 \\
-0.239 \\
+0.521 \\
-0.626 \\
+1.000\end{array}$ \\
\hline
\end{tabular}

Table 6.2: Binned survival probability parameters extracted from the SNO-LETA day/night analysis. The best-fit point gave $\chi^{2} / \mathrm{ndf}=56.05 /(57-12)=1.24$. The correlation coefficients are given in the lower part of the table.

\section{Discussion}

As the unconstrained CC and ES spectra in Figure 5.3, the day and night curves do not differ from each other by more than the fit uncertainties, for both the analytic and binned fits, over the whole range in $E_{\nu}$. Therefore it is not possible to claim experimental evidence for distortions of $P_{e e}\left(E_{\nu}\right)$ due to the matter in the Earth. The analytic and binned fits both agree with the latter conclusion.

The precision obtained is consistent with the acceptance of SNO versus $E_{\nu}$; uncertainties increase rapidly at energies below $6 \mathrm{MeV}$ and above $13 \mathrm{MeV}$, as expected 
from the verifications of Subsection 6.2.3. Because of these features, the extracted survival probability functions are mostly consistent with constants, which is reflected in the uncertainties of the parameters of the polynomial functions. The uncertainty in the $p_{0}$ parameter is the most meaningful indicator of the precision on $P_{e e}$. The latter uncertainty was $8.8 \%$ and $8.4 \%$ for the day and night functions, respectively. These uncertainties can be further reduced by replacing the survival probability functions by a single scale or by replacing the day/night curves by an average curve. However such changes would not allow one to see evidence of spectral distortions based on $P_{e e}$ or to infer possible day/night effects. Hence the survival probabilities extracted with the SNO-LETA data are slowly-decreasing functions with respect to $E_{\nu}$ and are fully compatible with flat lines.

A survival probability inconsistent with a flat line would confirm the energy dependence of neutrino flavor change in a model-independent way. Unfortunately the SNO acceptance is limited by statistics due to the cut-offs of the cross-sections and detector threshold at low-energy and by the end-point of the $\nu\left({ }^{8} \mathrm{~B}\right)$ spectrum at highenergy. In Subsection 6.3.2, the survival probability functions are extracted with the addition of other experimental data to investigate further the features observed with the SNO-LETA data.

\subsubsection{Results from Solar Neutrino Experiments}

The SNO experiment is only sensitive to high-energy (HE) neutrinos, namely the $\nu\left({ }^{8} \mathrm{~B}\right)$ 's and $\nu$ (hep)'s, and constrains the survival probability at $E_{\nu} \gtrsim 6 \mathrm{MeV}$. Other solar neutrino experiments, introduced in Chapter 1, have different sensitivities to $E_{\nu}$ down to energies as low as $0.233 \mathrm{MeV}$. The goal of a global solar survival probability 
analysis is therefore to combine, first, all the HE data to confirm the non-observation of the day/night effect at $\mathrm{HE}$, and second, to combine the HE with the low-energy (LE) data for an accurate extrapolation of the survival probability at LE. It will verify if the trend seen in Figure 6.6 stands since it is expected that Earth matter effects would be observed at HE while the Sun matter-induced transition would be at LE.

\section{Global Fit}

The global solar survival probability fit was performed with the analytic function only. The data that were included in the fit are described here. The rate experiments consist in three fractional rates integrated over all neutrino energies: the results from Homestake [13], combined gallium experiments [16], and Borexino [39] were included, with their uncertainties. The Homestake flux is composed of seven $\nu_{i}$ components, the gallium experiments of eight, being dominated by the LE $\nu(\mathrm{pp})$ 's, and the Borexino experiment is only sensitive to the second line of the $\nu\left({ }^{7} \mathrm{Be}\right)$ 's shown in Figure 1.2.

The SK data consisted of the measured ES spectra from the first two phases of the experiment $[19,20]$. The 44 SK-I fractional rates are given in terms of day/mantle/core zenith orientations at the Earth. The 33 SK-II rates are provided as day/night spectra. The parameters of the SK detector response functions, similar to Equations (6.3), were taken from [19, 20].

Finally, the SNO-NCD results [27], in the form of the three average fluxes (CC, ES, and NC) obtained from a constrained fit with a threshold of $T_{\mathrm{eff}}^{0}=6 \mathrm{MeV}$ to the SNO data taken in the third phase were included. In total there were $N_{\mathrm{LE}}+N_{\mathrm{SK}}+N_{\mathrm{SNO}}=$ $3+77+60=140$ fractional rates and 85 systematic uncertainties, in which the uncertainty in the shape of the $\nu\left({ }^{8} \mathrm{~B}\right)$ spectrum affects all experiments simultaneously. 
The rates and normalizations, used to build the $F_{n}^{\mathrm{ex}}$, are explicitly given in Table C.1 of Appendix C.

\section{High Energy Neutrinos}

The first combination consists of SNO-LETA and SNO-NCD with the SK spectra. The SK-I spectra were tagged so that the day-time bin contributed to the day-time survival probability and the mantle/core bins contributed to the night-time function. Similar to SNO, the measured rates, irrespective of the units, were divided by the expected rate without oscillation such that differences between the detector simulations and the analytic method canceled. The systematic uncertainties were assumed to be fully correlated in all rates for a given experiment, except when specified in the publications, and uncorrelated with other systematic uncertainties. Model parameters were treated as in Subsection 6.3.1; the flux normalizations $f_{8_{\mathrm{B}}}$ and $f_{\text {hep }}$ and the $s 8_{\mathrm{B}}$ uncertainty affected all SNO and SK rates simultaneously.

The combined fit gave $\chi_{\min }^{2}=126.08$ for 126 degrees of freedom, with $f_{8_{\mathrm{B}}}=$ $0.934 \pm 0.031$ and $f_{\text {hep }}=0.996 \pm 0.155$. Figure 6.7 a shows the result of the day/night analytic survival probability fit in the range $E_{\nu} \in[6,13] \mathrm{MeV}$. Compared to the results of Figure 6.6b, both survival probability curves have shifted down near $E_{\nu} \sim 6 \mathrm{MeV}$ and have smaller uncertainties over the whole range in $E_{\nu}$. Although the day and night curves are shifted in the range $E_{\nu} \in[7,10] \mathrm{MeV}$, the $1 \sigma$-bands overlap in the most sensitive region of both experiments. Therefore the non-observation of a day/night effect in HE $\nu$ 's still holds. This conclusion agrees with previous publications from both SNO and SK collaborations on the day/night asymmetry [19, 20, 28, 30, 137]. 


\section{Average and Degenerated Probabilities}

The solar global fit including all experimental data was possible only after making a few simplifications explained here. First, the only experiments for which a day/night configuration is possible are $\mathrm{SNO}$ and SK due to their real-time observation of neutrino events. The fit result from Figure 6.7a showed that the asymmetry was nonexistent, hence the day/night separation was dropped for a global average which eases the treatment of the rest of the experimental data. Second, since the survival probability fit is independent of oscillation models, a single survival probability function was assumed for all eight types of $\nu$ 's, irrespective of their origin in the Sun. Hence all neutrino types were degenerate in the average analysis. The effective neutrino spectrum seen at the Earth is the sum of the spectra shown in Figure 1.2 weighted by the unknown $\bar{P}_{e e}^{\odot}$ function and by the convolution effect of each detector. Finally, the eight neutrino flux scales were allowed to vary in the fit with penalty terms equivalent to their $3 \sigma \mathrm{SSM}$ uncertainties except for $f_{8_{\mathrm{B}}}$ that is well constrained by the $\mathrm{NC}$ flux measurement of SNO.

The global fit gave $\chi_{\min }^{2}=130.82$ for 128 degrees of freedom, with $f_{8_{\mathrm{B}}}=0.938 \pm$ $0.031, f_{\text {hep }}=0.927 \pm 0.458, f_{\mathrm{pp}}=0.99 \pm 0.03$, and all other scales were consistent with their input values. Figure $6.7 \mathrm{~b}$ shows the survival probability function, $\bar{P}_{e e}^{\odot}$, obtained from the combined fit of all solar neutrino data in the averaged and degenerated context. The result is a smooth decreasing function, from $\bar{P}_{e e}^{\odot}(1 \mathrm{MeV})=0.539 \pm 0.042$, to $\bar{P}_{e e}^{\odot}(9.5 \mathrm{MeV}) \equiv p_{0}=0.320 \pm 0.018$, with a slight increasing trend for $E_{\nu}>10 \mathrm{MeV}$. The bridge between the LE and HE experiments, $E_{\nu} \in[2,6] \mathrm{MeV}$, has a perceptible wider uncertainty band due to the lack of sensitive data in that range. Nevertheless, the conclusion is that the world-average curve is smooth and that the LE and HE 
neutrinos do not have the same survival probability since the difference between two consecutive order of magnitudes in $E_{\nu}$ is:

$$
\Delta \bar{P}_{e e}^{\odot}=\bar{P}_{e e}^{\odot}(1 \mathrm{MeV})-\bar{P}_{e e}^{\odot}(10 \mathrm{MeV})=0.220 \pm 0.045
$$

which is approximately $4.9 \sigma$ away from zero.

\subsubsection{Conservation of Unitarity}

Complementary to the determination of $P_{e e}$ where the sensitivity of the CC reactions to $\nu_{e}$ 's compared to the total flux given by SNO's NC drives the survival probability curve and the scale of the fluxes, the formalism was modified such that $P_{e e}$ and $P_{e a}$, the transition probability $P\left(\nu_{e} \rightarrow \nu_{\mu, \tau}\right)$, were extracted simultaneously without constraints.

The sum $P_{e e}+P_{e a}$ is therefore not constrained to be exactly one as in Equation (1.10) in order to test the unitarity of the probabilities, but is still determined by SNO's NC. The $P_{e e}$ component is determined by all CC and ES $\nu_{e}$ rates, while the $P_{e a}$ component by the $\mathrm{SK}, \mathrm{SNO}$, and Borexino part of the ES rates sensitive to $\nu_{\mu, \tau}$ 's. The main advantage of this configuration is that it allows the precision of the SNO NC rate to impact the precision on $P_{e e}\left(E_{\nu}\right)$ directly. The number of probability parameters doubles but the flux scales are dropped, hence the fitter only has eight parameters to evaluate from 140 fractional rates.

The fit returned $\chi_{\min }^{2}=130.83$ for 132 degrees of freedom which is slightly better than the solar global fit presented in Subsection 6.3.2. Figure 6.8 shows the survival and transition probability curves, with the sum calculated from the two functions. 
SNO+SK Survival Probabilities

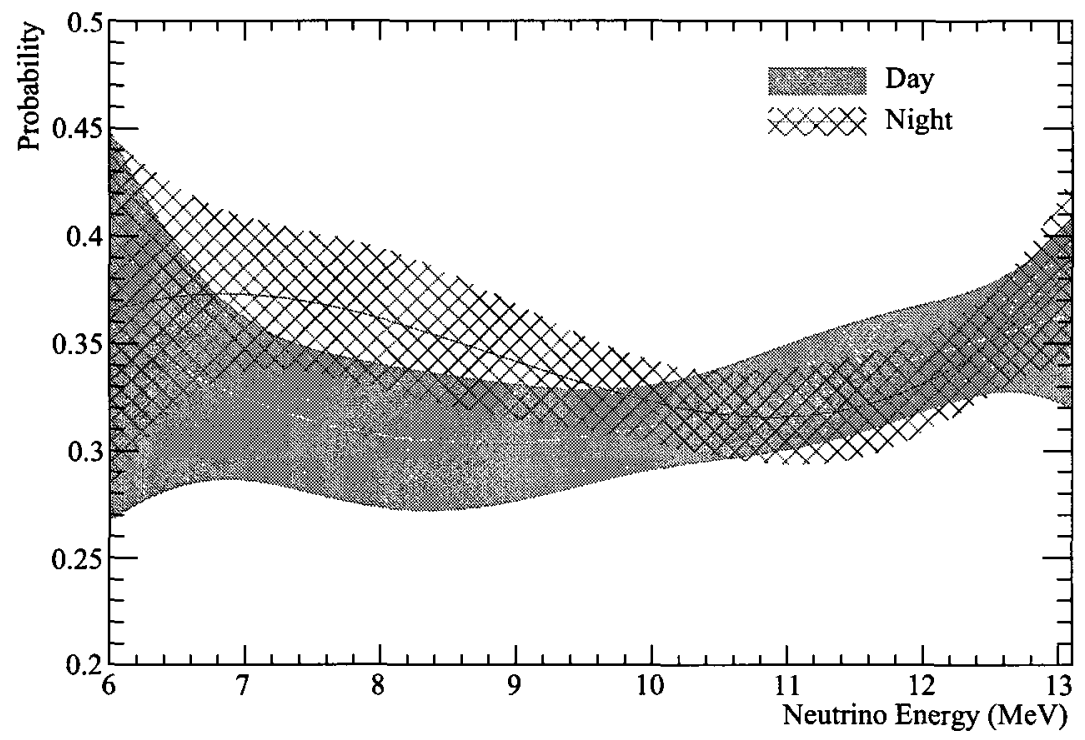

(a) Day/night curves from SNO+SK.

Solar Neutrino Average Survival Probabilities

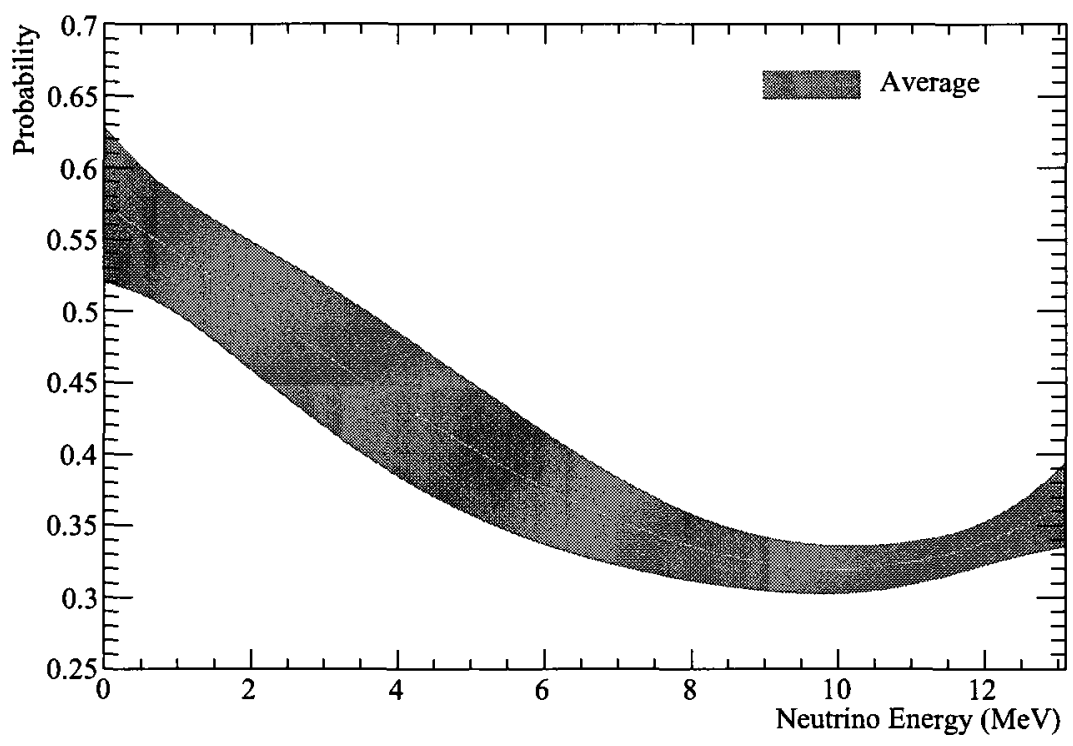

(b) Average curve from all solar neutrino experiments.

Figure 6.7: Survival probability function extracted from solar all neutrino experiments, including the contribution of the SNO-LETA day/night combined fit of the $\mathrm{D}_{2} \mathrm{O}$ and salt phases. (a) Second-generation experiments sensitive to $\nu\left({ }^{8} \mathrm{~B}\right)$ 's: SNO-LETA, SNO-NCD, SK-I, and SK-II, gave $\chi^{2} / \mathrm{ndf}=126.08 / 126=1.001$. (b) All solar experimental data (SNO, SK, Homestake, Gallium, and Borexino) were used without distinction of zenith orientation or neutrino type and yielded $\chi^{2} / \mathrm{ndf}=130.82 / 128=1.022$. 


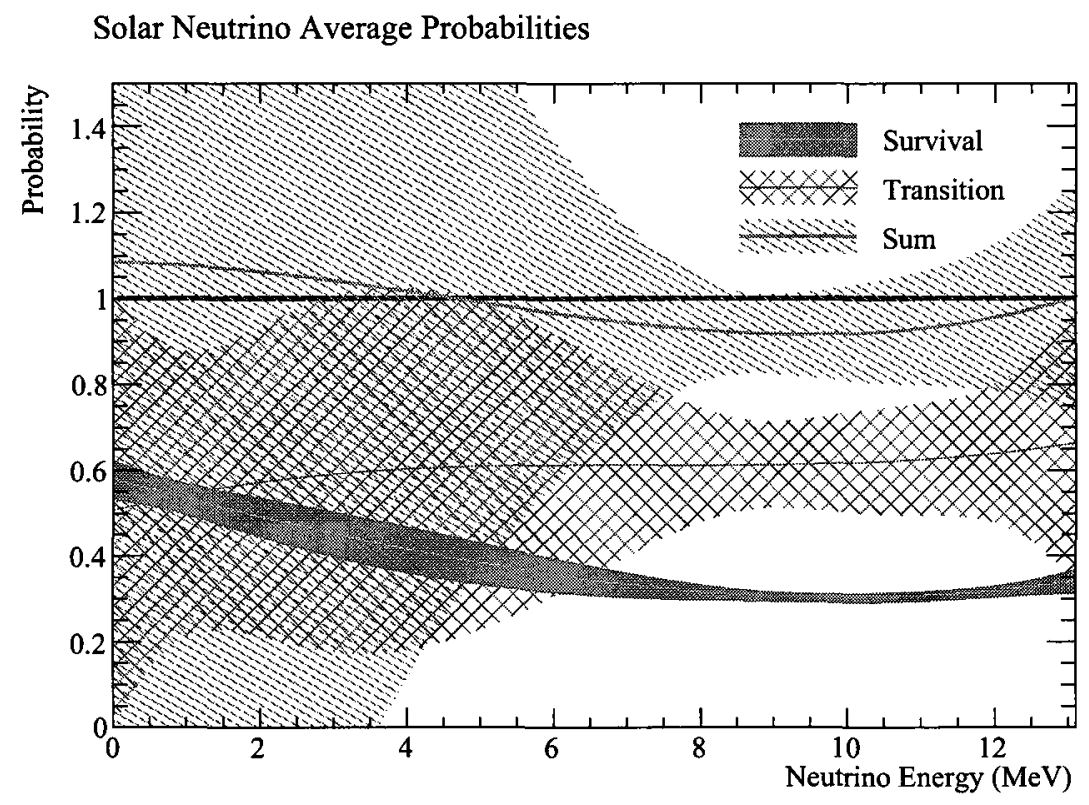

Figure 6.8: Survival, transition, and total probability functions extracted from all solar neutrino experiments, including the contribution of the SNO-LETA day/night combined fit of the $\mathrm{D}_{2} \mathrm{O}$ and salt phases. All experimental data are used without distinction of zenith orientation or neutrino type. The minimum was $\chi^{2} / \mathrm{ndf}=130.83 / 132=0.991$.

The error band on the sum takes into account the cross-correlations between the $P_{e e}$ and $P_{e a}$ functions. Unitarity is conserved in the whole range of $E_{\nu}$ basically due to the large uncertainties on the $P_{e a}$ curve. The most precise range is where the NC measurement from SNO constrains the total $\nu$ flux. The decrease of the total probability near $E_{\nu}=9.5 \mathrm{MeV}$ with respect to the unitarity value is due to SNO's NC that predicts $P_{e e}+P_{e a}=f_{8_{\mathrm{B}}} \approx 0.9$. One can clearly see from the $P_{e a}$ curve the zones where the ES rates were measured with precision: near $1 \mathrm{MeV}$ in Borexino, and for $E_{\nu} \in[8,12] \mathrm{MeV}$ in SK and SNO. The scale of $P_{e a}$ was found to be $0.614 \pm 0.106$, while all other parameters of $P_{e a}$ were consistent with zero. The precision achieved on $p_{0}$ in the $P_{e e}$ function was of the order of $4 \%$ using this method. 


\begin{tabular}{|l|c||cccc|}
\hline \hline Configuration & Figure & $p_{0}$ & $\begin{array}{c}p_{1} \\
\left(\times 10^{-1} \mathrm{MeV}^{-1}\right)\end{array}$ & $\begin{array}{c}p_{2} \\
\left(\times 10^{-2} \mathrm{MeV}^{-2}\right)\end{array}$ & $\begin{array}{c}p_{3} \\
\left(\times 10^{-3} \mathrm{MeV}^{-3}\right)\end{array}$ \\
\hline \hline SNO-LETA-D & 6.6 & $0.315 \pm 0.028$ & $+0.135 \pm 0.245$ & $+0.776 \pm 0.716$ & $-2.215 \pm 3.259$ \\
SNO-LETA-N & 6.6 & $0.321 \pm 0.027$ & $+0.007 \pm 0.228$ & $+1.471 \pm 0.740$ & $-3.015 \pm 3.158$ \\
\hline SNO+SK-D & $6.7 \mathrm{a}$ & $0.306 \pm 0.022$ & $+0.074 \pm 0.208$ & $+0.440 \pm 0.495$ & $-0.567 \pm 2.551$ \\
SNO+SK-N & $6.7 \mathrm{a}$ & $0.331 \pm 0.022$ & $-0.191 \pm 0.193$ & $+0.330 \pm 0.452$ & $+1.723 \pm 2.339$ \\
\hline Solar-A & $6.7 \mathrm{~b}$ & $0.320 \pm 0.018$ & $-0.041 \pm 0.076$ & $+0.397 \pm 0.239$ & $+0.167 \pm 0.322$ \\
Solar-Unitarity $P_{e e}$ & 6.8 & $0.301 \pm 0.011$ & $-0.035 \pm 0.094$ & $+0.358 \pm 0.250$ & $+0.095 \pm 0.345$ \\
Solar-Unitarity $P_{e a}$ & 6.8 & $0.614 \pm 0.106$ & $+0.036 \pm 0.829$ & $+0.181 \pm 2.451$ & $+0.276 \pm 3.272$ \\
\hline \hline
\end{tabular}

Table 6.3: Summary of the analytic survival probability parameters. The parameters refer to $P_{e e}\left(E_{\nu}\right)$ of Equation (6.8) with $E_{0}=9.5 \mathrm{MeV}$. The zenith orientations are day (D), night $(\mathrm{N})$ or the weighted experimental average $(\mathrm{A})$.

\subsubsection{Summary}

A model-independent determination of the survival probability of solar electron neutrinos was presented for the first time from a global solar analysis. The survival probability was extracted from the SNO-LETA unconstrained fits and from the combination with five other solar neutrino experiments, including the result from the SNO-NCD phase. The general result, verified and obtained with two distinct parameterizations, was a smooth and slow-decreasing function of $E_{\nu}$ with strong evidence that low- and high-energy solar neutrinos are affected by different mechanisms before they are detected on Earth. The parameters of the analytic probability functions that were extracted in this chapter are summarized in Table 6.3. The precision on the $p_{0}$ parameter, the scale of the probability function, was enhanced by the incremental addition of precise rates into the fit that led to an uncertainty of $5.6 \%$ when enforcing unitarity and $3.6 \%$ without the latter constraint.

While other parameterizations of the analytic survival probability might give bet- 
ter results, the polynomial form of Equation (6.8) was useful at extracting the scale of the functions in cases where the dependence in $E_{\nu}$ was weak at high energy. Future measurements that should improve the extraction of the survival probability are a forthcoming simultaneous three-phase fit of the three SNO signals [36], the determination of the total solar neutrino flux at low energy, and perhaps the design of new experiments sensitive to single, unmeasured, neutrino lines that would provide sensitivity in the range of $E_{\nu}$ between the first and second generation of solar experiments.

The analysis of the survival probability continues in Chapter 7 where the goal is to interpret the fitted survival probabilities in terms of oscillation parameters and confirm the physical mechanism that describes the experimental data. Particularly, the survival probability is calculated based on neutrino oscillation parameters which are then extracted from the comparison of the predicted and measured rates, in both the $2 \nu$ - and full $3 \nu$-flavor contexts. Then, in the conclusion of this thesis, the comparison of the measured and predicted probabilities is made to validate the hypothesis at the origin of the theoretical framework that dictates how neutrinos behave in general. 


\section{CHAPTER 7}

\section{Extraction of the Neutrino Oscillation Parameters}

The analysis presented in this chapter is the extraction of the theoretical neutrino oscillation parameters under the survival probability curves found in Chapter 6 . The mechanism at the source of neutrino oscillation has been introduced in Chapter 1 and is explained in more detail. The numerical calculation of the survival probabilities is also described. The oscillation parameters are extracted from the solar and reactor neutrino data in the context of two- and three-flavor neutrino mixing models, for the first time, because the improvement of the uncertainties in the LETA allows one to study second-order effects due to $\theta_{13}$.

\subsection{Survival Probability Formulae}

This section introduces the formulae needed to calculate the survival probabilities for a given detector location from a wide range of oscillation parameter values. Subsection 7.1.1 completes the theoretical introduction of Chapter 1, while Subsections 7.1.2 and 7.1.3 give the explicit dependence of $P_{e e}$ on the oscillation parameters in the $2 \nu$ and $3 \nu$ models, respectively. 


\subsubsection{Phenomenology of Neutrino Oscillation}

Neutrino flavor change, or oscillation, is generated by the interference patterns of the massive eigenstates and can be enhanced in the presence of matter. The Hamiltonian in flavor space, $H_{f}$, was given in Equation (1.11) with the mixing matrix:

$$
\begin{aligned}
U & =R_{23} \times R_{13} \times R_{12} \\
& =\left(\begin{array}{ccc}
1 & 0 & 0 \\
0 & c_{23} & s_{23} \\
0 & -s_{23} & c_{23}
\end{array}\right) \times\left(\begin{array}{ccc}
c_{13} & 0 & s_{13} e^{-i \delta} \\
0 & 1 & 0 \\
-s_{13} e^{i \delta} & 0 & c_{13}
\end{array}\right) \times\left(\begin{array}{ccc}
c_{12} & s_{12} & 0 \\
-s_{12} & c_{12} & 0 \\
0 & 0 & 1
\end{array}\right) \\
& =\left(\begin{array}{ccc}
c_{12} c_{13} & s_{12} c_{13} & s_{13} e^{-i \delta} \\
-s_{12} c_{23}-c_{12} s_{23} s_{13} e^{i \delta} & c_{12} c_{23}-s_{12} s_{23} s_{13} e^{i \delta} & s_{23} c_{13} \\
s_{12} s_{23}-c_{12} c_{23} s_{13} e^{i \delta} & -c_{12} s_{23}-s_{12} c_{23} s_{13} e^{i \delta} & c_{23} c_{13}
\end{array}\right),
\end{aligned}
$$

where $c_{j k} \equiv \cos \theta_{j k}$ and $s_{j k} \equiv \sin \theta_{j k}$. The mixing matrix $U$ was modified from the above general form for the two specific cases studied in this thesis. First, the effective $2 \nu$ oscillation model only requires two active flavor states $\left(\nu_{e}, \nu_{a=\mu, \tau}\right)$ and massive states $\left(\nu_{1}, \nu_{2}\right)$. In that case the matrix reduces to $U=R_{12}$, corresponding to $\theta_{23}=\theta_{13}=0$. Second, the $3 \nu$ oscillation model, which takes into account all possible transitions, was simplified for solar neutrinos. Because the latter are produced as $\nu_{e}$ 's in the Sun and detected as either $\nu_{e}$ 's or $\nu_{a}$ 's on Earth, the effect of $\theta_{23}$, mainly responsible for $\nu_{\mu} \leftrightarrow \nu_{\tau}$ transitions, is irrelevant. In addition, since the additional effect of $\theta_{13}$ is expected to be small, the CP-violating phase was removed by setting $\delta=0$.

The mass matrix $M$ depends on two independent $\Delta m^{2}$ parameters. It was kept 
intact for the $3 \nu$ analysis, and reduced to a $2 \times 2$ matrix for the $2 \nu$ analysis by keeping $\Delta m_{21}^{2}$ only. In all cases, normal hierarchy of the mass eigenstates is assumed,

$m_{3}>m_{2}>m_{1}$, such that the mass differences obey the rule $\Delta m_{32}^{2}+\Delta m_{21}^{2}-\Delta m_{31}^{2}=0$.

The order of magnitude of the current measured values [2] allows one to approximate $\Delta m_{31}^{2} \simeq \Delta m_{32}^{2}$ because $\left|\Delta m_{32}^{2}\right| \gg\left|\Delta m_{21}^{2}\right|$.

The matrix $A_{\mathrm{CC}}$ takes into account the matter effects. It relies on the electron density function that was determined dynamically as a function of position in the Sun and Earth from the inputs described in Subsection 7.2.1. The density was the same in both the $2 \nu$ and $3 \nu$ models, but affected them in different and subtle ways.

Hence, in the $3 \nu$ oscillation model, the parameters describing the $P_{e e}\left(E_{\nu}\right)$ function for solar neutrinos are, in order of importance, $\theta_{12}, \Delta m_{21}^{2}, \theta_{13}$, and $\Delta m_{31}^{2}$. For experiments sensitive to neutrinos from terrestrial sources, near the detector, the survival probabilities are accurately calculated using the general formula of Equation (1.9) without the effect of matter.

\section{Sun, Vacuum and Earth Components}

Solar neutrinos travel through the Sun, vacuum, and Earth, before they reach a detector (d) in the state $\left|\nu^{\mathrm{d}}\right\rangle$. At the origin, in the Sun, the initial condition is $\left|\nu^{\odot}(0)\right\rangle=\left|\nu_{e}\right\rangle$. At the surface of the Sun, the state is a superposition of flavor states:

$$
\left|\nu^{\odot}\right\rangle=\sum_{\alpha} \beta_{\alpha}\left|\nu_{\alpha}\right\rangle
$$

where $\alpha=e, \mu, \tau$. Because of the distribution of the source of $\nu_{e}$ 's in the Sun, the Sun-Earth variable distance, and detector resolution effects, the states are detected on Earth in an incoherent manner. This incoherence allows one to drop the vacuum 
propagation calculation and perform an average of the phases $\Delta m_{k j}^{2} L / 2 E_{\nu}$ in the vacuum survival probability of Equation (1.9). Effectively, this corresponds to averaging the states at the surface of the Sun such that the probabilities before entering the Earth are written $\left|\beta_{\alpha}\right|^{2} \underset{\text { avg }}{\longrightarrow} \bar{P}_{e \alpha}^{\odot}$, where the bar notation $(-)$ indicates the phase average was obtained.

From the latter incoherent states, the propagation must continue through the Earth. After its passage through the Earth, the state has the form

$$
\left|\nu^{\mathrm{d}}\right\rangle=\sum_{\alpha} \beta_{\alpha}\left|\Psi^{\alpha}\right\rangle
$$

where the Earth transmission functions, $\left|\Psi^{\alpha}\right\rangle$, describe the state, independently of the Sun function coefficients $\beta_{\alpha}$, from the Earth entry point to the detector:

$$
\left|\Psi^{\alpha}\right\rangle=b_{e}^{\alpha}\left|\nu_{e}\right\rangle+b_{\mu}^{\alpha}\left|\nu_{\mu}\right\rangle+b_{\tau}^{\alpha}\left|\nu_{\tau}\right\rangle
$$

The survival probability at the detector, $P^{\mathrm{d}}$, is found from the wave function coefficients:

$$
P^{\mathrm{d}}=\left|\left\langle\nu_{e} \mid \nu^{\mathrm{d}}\right\rangle\right|^{2}
$$

which, after expansion, gives a complicated function of the complex coefficients $\beta_{\alpha}$ and $b_{\gamma}^{\alpha}$. The latter expansion, however, leads to an expression where the Sun and Earth coefficients are decoupled [138], which allows the probabilities to be calculated separately in the Sun and Earth and assembled thereafter with dedicated formulae to obtain $P^{\mathrm{d}}$. The key in finding suitable formulae is to select the appropriate initial conditions in the Earth calculation. In general the three-flavor probabilities require 
that at least two initial conditions are assumed at the Earth, leading to Earth probabilities $P_{\mathrm{E} 1}^{\oplus}$ and $P_{\mathrm{E} 2}^{\oplus}$. Subsections 7.1.2 and 7.1.3 discuss how to combine the separate Sun and Earth probabilities in the $2 \nu$ and $3 \nu$ contexts, respectively.

\subsubsection{Two-State Formula}

The $2 \nu$-model assumes two flavor $\left(\nu_{e}, \nu_{a}\right)$ and mass $\left(\nu_{1}, \nu_{2}\right)$ eigenstates. Thus, there is only one Earth initial condition required but to avoid averaging the phases arising from the Earth coefficients numerically, two $P^{\oplus}$ were calculated. The first was $P_{\mathrm{E} 1}^{\oplus}=P\left(\nu_{e} \rightarrow \nu_{e}\right)$ and the second was obtained from an initial, equal mixture $P_{\mathrm{E} 2}^{\oplus}=P\left(\frac{1}{2}\left(\nu_{e}+\nu_{a}\right) \rightarrow \nu_{e}\right)$. With those conditions, and the survival probability from the Sun, $\bar{P}_{e e}^{\odot}$, the formula was obtained from the technique outlined in $[139,140]$ :

$$
\begin{aligned}
& P_{\mathrm{E} 1}^{\oplus} \equiv P_{\nu_{e} \rightarrow \nu_{e}}^{\oplus} \\
& P_{\mathrm{E} 2}^{\oplus} \equiv P_{\nu_{e}+\nu_{a} \rightarrow \nu_{e}}^{\oplus} \\
& P_{2 \nu}^{\mathrm{d}}=1+2 \bar{P}_{e e}^{\odot} P_{\mathrm{E} 1}^{\oplus}-\bar{P}_{e e}^{\odot}-P_{\mathrm{E} 1}^{\oplus}-\frac{1}{2}\left(2 \bar{P}_{e e}^{\odot}-1\right)\left(2 P_{\mathrm{E} 2}^{\oplus}-1\right) \tan \left(2 \theta_{12}\right),
\end{aligned}
$$

where the three quantities $\bar{P}_{e e}^{\odot}, P_{\mathrm{E} 1}^{\oplus}$, and $P_{\mathrm{E} 2}^{\oplus}$ all depend implicitly on $\theta_{12}$ and $\Delta m_{21}^{2}$, the only two parameters of the effective $2 \nu$-model.

\subsubsection{Three-State Formula}

The $3 \nu$-model involves all three flavor $\left(\nu_{e}, \nu_{\mu}, \nu_{\tau}\right)$ and mass $\left(\nu_{1}, \nu_{2}, \nu_{3}\right)$ eigenstates. There are many possible Earth initial conditions that can be calculated. Computationally, however, the calculation of probabilities resulting from an initial mass eigenstate to be detected as a pure flavor eigenstate removes the need to average over 
Earth phases left in the expansion of the flavor eigenstate. Thus, for practical reasons, the choices were reduced to $P\left(\nu_{1} \rightarrow \nu_{e}\right), P\left(\nu_{2} \rightarrow \nu_{e}\right)$, and $P\left(\nu_{3} \rightarrow \nu_{e}\right)$.

In addition, for solar $\nu$ 's, the irrelevance of 2-3 interferences allows one to set $\theta_{23}$ to any value. Conveniently, $\theta_{23}$ disappears from the mixing matrix $U$ when it is zero or $\pi / 2$. These aesthetic choices however do not allow any arbitrary combinations of $P_{\mathrm{E} 1}^{\oplus}$ and $P_{\mathrm{E} 2}^{\oplus}$; unitarity constraints prohibit the choices of extreme values of the matrix elements $U_{\alpha k}$. The value $\theta_{23}=\pi / 2$ was set, but the transitions from mass to flavor eigenstates that involved terms with $\theta_{23}$ alone in $U$ were carefully avoided. Equation (7.3) shows that the third column, specifically the $U_{\mu 3}$ and $U_{\tau 3}$ elements, contains such terms. Thus the option $P\left(\nu_{3} \rightarrow \nu_{e}\right)$ was eliminated from the choices because $\theta_{23}=\pi / 2$. Therefore the transitions $\nu_{1} \rightarrow \nu_{e}$ and $\nu_{2} \rightarrow \nu_{e}$ were selected as initial conditions, defining $P_{\mathrm{E} 1}^{\oplus}=P\left(\nu_{1} \rightarrow \nu_{e}\right)$ and $P_{\mathrm{E} 2}^{\oplus}=P\left(\nu_{2} \rightarrow \nu_{e}\right)$.

With the latter Earth initial conditions, the formula was obtained from the method described in $[141,142]$ with the coefficients $a_{\mathrm{E} 1}^{\alpha}=\left|U_{\alpha 1}\right|^{2}$ and $a_{\mathrm{E} 2}^{\alpha}=\left|U_{\alpha 2}\right|^{2}$ to weight the $P_{\mathrm{E} 1}^{\oplus}$ and $P_{\mathrm{E} 2}^{\oplus}$ components:

$$
\begin{aligned}
P_{\mathrm{E} 1}^{\oplus} \equiv & P_{\nu_{1} \rightarrow \nu_{e}}^{\oplus} \\
P_{\mathrm{E} 2}^{\oplus} \equiv & P_{\nu_{2} \rightarrow \nu_{e}}^{\oplus} \\
P_{3 \nu}^{\mathrm{d}}=\frac{1}{A}[ & \bar{P}_{e e}^{\odot}\left(P_{\mathrm{E} 1}^{\oplus}\left(a_{\mathrm{E} 2}^{\mu}-a_{\mathrm{E} 2}^{\tau}\right)+P_{\mathrm{E} 2}^{\oplus}\left(a_{\mathrm{E} 1}^{\tau}-a_{\mathrm{E} 1}^{\mu}\right)+a_{\mathrm{E} 2}^{\tau} a_{\mathrm{E} 1}^{\mu}-a_{\mathrm{E} 1}^{\tau} a_{\mathrm{E} 2}^{\mu}\right) \\
& \quad+\bar{P}_{e \mu}^{\odot}\left(P_{\mathrm{E} 1}^{\oplus}\left(a_{\mathrm{E} 2}^{\tau}-a_{\mathrm{E} 2}^{e}\right)+P_{\mathrm{E} 2}^{\oplus}\left(a_{\mathrm{E} 1}^{e}-a_{\mathrm{E} 1}^{\tau}\right)+a_{\mathrm{E} 2}^{e} a_{\mathrm{E} 1}^{\tau}-a_{\mathrm{E} 1}^{e} a_{\mathrm{E} 2}^{\tau}\right) \\
& \left.\quad+\bar{P}_{e \tau}^{\odot}\left(P_{\mathrm{E} 1}^{\oplus}\left(a_{\mathrm{E} 2}^{e}-a_{\mathrm{E} 2}^{\mu}\right)+P_{\mathrm{E} 2}^{\oplus}\left(a_{\mathrm{E} 1}^{\mu}-a_{\mathrm{E} 1}^{e}\right)+a_{\mathrm{E} 1}^{e} a_{\mathrm{E} 2}^{\mu}-a_{\mathrm{E} 2}^{e} a_{\mathrm{E} 1}^{\mu}\right)\right] \\
A= & \left(a_{\mathrm{E} 1}^{e}-a_{\mathrm{E} 1}^{\tau}\right)\left(a_{\mathrm{E} 2}^{\mu}-a_{\mathrm{E} 2}^{\tau}\right)-\left(a_{\mathrm{E} 1}^{\mu}-a_{\mathrm{E} 1}^{\tau}\right)\left(a_{\mathrm{E} 2}^{e}-a_{\mathrm{E} 2}^{\tau}\right) .
\end{aligned}
$$

After replacing the coefficients involving flavor $\tau$ using unitarity constraints, 
$\bar{P}_{e \tau}^{\odot}=1-\bar{P}_{e \mu}^{\odot}-\bar{P}_{e e}^{\odot}$ and $a^{\tau}=1-a^{\mu}-a^{e}$, and setting $\theta_{23}=\pi / 2$ allowed by the specific choice of $P_{\mathrm{E} 1}^{\oplus}$ and $P_{\mathrm{E} 2}^{\oplus}$, the formula becomes:

$$
\begin{aligned}
P_{3 \nu}^{\mathrm{d}}=\frac{1}{A}[ & \bar{P}_{e e}^{\odot}\left(P_{\mathrm{E} 1}^{\oplus}\left(3 a_{\mathrm{E} 2}^{\mu}-1\right)+P_{\mathrm{E} 2}^{\oplus}\left(1-3 a_{\mathrm{E} 1}^{\mu}\right)+a_{\mathrm{E} 1}^{\mu}-a_{\mathrm{E} 2}^{\mu}\right) \\
+ & \bar{P}_{e \mu}^{\odot}\left(P_{\mathrm{E} 1}^{\oplus}\left(1-3 a_{\mathrm{E} 2}^{e}\right)+P_{\mathrm{E} 2}^{\oplus}\left(3 a_{\mathrm{E} 1}^{e}-1\right)+a_{\mathrm{E} 2}^{e}-a_{\mathrm{E} 1}^{e}\right) \\
& \left.+P_{\mathrm{E} 1}^{\oplus}\left(a_{\mathrm{E} 2}^{e}-a_{\mathrm{E} 2}^{\mu}\right)+P_{\mathrm{E} 2}^{\oplus}\left(a_{\mathrm{E} 1}^{\mu}-a_{\mathrm{E} 1}^{e}\right)+a_{\mathrm{E} 1}^{e} a_{\mathrm{E} 2}^{\mu}-a_{\mathrm{E} 2}^{e} a_{\mathrm{E} 1}^{\mu}\right] \\
A= & 3\left(a_{\mathrm{E} 1}^{e} a_{\mathrm{E} 2}^{\mu}-a_{\mathrm{E} 2}^{e} a_{\mathrm{E} 1}^{\mu}\right)+a_{\mathrm{E} 2}^{e}+a_{\mathrm{E} 1}^{\mu}-a_{\mathrm{E} 1}^{e}-a_{\mathrm{E} 2}^{\mu} \\
a_{\mathrm{E} 1}^{e}= & c_{12}^{2} c_{13}^{2} \quad a_{\mathrm{E} 1}^{\mu}=c_{12}^{2} s_{13}^{2} \quad a_{\mathrm{E} 2}^{e}=s_{12}^{2} c_{13}^{2} \quad a_{\mathrm{E} 2}^{\mu}=s_{12}^{2} s_{13}^{2} .
\end{aligned}
$$

Similar to the expression of $P_{2 \nu}^{\mathrm{d}}$, the complicated dependence on the mixing angles and mass differences is hidden in the coefficients $\bar{P}_{e e}^{\odot}, \bar{P}_{e \mu}^{\odot}, P_{\mathrm{E} 1}^{\oplus}, P_{\mathrm{E} 2}^{\oplus}, a_{\mathrm{E} 1}^{\alpha}$, and $a_{\mathrm{E} 2}^{\alpha}$. Hence the survival probability formulae of Equations (7.8) and (7.10) are non-trivial functions of the oscillation parameters. Section 7.2 explains how the above coefficients were calculated numerically before they were used in the expressions of $P_{2 \nu}^{\mathrm{d}}$ and $P_{3 \nu}^{\mathrm{d}}$.

\subsection{Calculation of the Survival Probability}

The package of formulae presented in Section 7.1 are suited to a parameter-scan of the survival probabilities. Survival probability tables were constructed independently for the Sun and Earth. Each table is a function of the oscillation parameters $\tan ^{2} \theta_{12}$, $\Delta m_{21}^{2} / E_{\nu}$, and $\sin ^{2} \theta_{13}$. The $\Delta m_{31}^{2}$ parameter was fixed to a single effective value since its effect in $P_{3 \nu}^{\mathrm{d}}$ is suppressed compared to the other three parameters. The strategy was to scan the logarithmic parameters linearly with a fine step such that interpolation errors were minimized. This was especially important for $\Delta m_{21}^{2}$ which 


\begin{tabular}{|l||c|c|c|}
\hline \hline Parameter & \multicolumn{2}{|c|}{ Range } & Step Size \\
\hline$p$ & {$\left[p_{\min }, p_{\max }\right]$} & {$\left[\log _{10}\left(p_{\min }\right), \log _{10}\left(p_{\max }\right)\right]$} & $\Delta \log _{10}(p)$ \\
\hline \hline $\tan ^{2} \theta_{12}$ & {$\left[10^{-4}, 10^{1}\right]$} & {$[-4,+1]$} & 0.05 \\
$\Delta m_{21}^{2}$ & {$\left[10^{-12}, 10^{-3}\right]$} & {$[-12,-3]$} & 0.05 \\
$\sin ^{2} \theta_{13}$ & {$\left[10^{-5}, 10^{-0.6}\right]$} & {$[-5,-0.6]$} & 0.1 \\
$\Delta m_{31}^{2}$ & $10^{-3}$ & -3 & - \\
\hline
\end{tabular}

Table 7.1: Limits and step sizes in the neutrino oscillation parameter scan. The effective range of $\log _{10}\left(\Delta m_{21}^{2} / E_{\nu}\right)$ extends to $[-13,-2]$. The value of $\Delta m_{31}^{2}$ is effective at $E_{\nu}=$ $10 \mathrm{MeV}$.

appears in the form of $\Delta m_{21}^{2} / E_{\nu}$ in Equation (1.11), so that all $\Delta m_{21}^{2}$ and $E_{\nu}$ values could be retrieved at once from the tables. Table 7.1 lists the ranges and step sizes of the oscillation parameter scan. The mixing angles were parameterized using the trigonometric functions $\tan ^{2} \theta_{12}$ and $\sin ^{2} \theta_{13}$, respectively. The function $\tan ^{2} \theta_{12}$ was chosen so that the range $\theta_{12} \in\left[0, \frac{\pi}{2}\right]$ was covered to remove the ambiguity on the sign of $\Delta m_{21}^{2}$ in matter-enhanced oscillation [143].

\section{Numerical Integration}

The inclusion of matter effects in the survival probability calculation involves the numerical integration of a system of coupled differential equations:

$$
i \frac{d}{d x} \psi_{\alpha}(x)=H_{f} \psi_{\alpha}(x)
$$

where $x$, equivalent to the time $t$, is the position along the propagation direction and $\psi_{\alpha}(x)$ a vector containing the real and imaginary coefficients of the wave function in flavor space. The system was solved for each new value of $x$ as the wave function was propagated from the starting to the end point. The probabilities were then calculated 
from the norm of the wave function coefficients.

The integration was performed with the adaptative Runge-Kutta algorithm [106, 120]. Comparisons with similar methods such as the fourth-order Runge-Kutta [106, 144], Burlisch-Stoer [106, 145], and independent analytic approximations [141, 146] have shown that the numerical precision of the survival probability is better than $0.1 \%$ which is negligible compared to all other sources of uncertainties on the rates used in the oscillation analysis.

\subsubsection{Propagation in the Sun}

The calculation of the survival probabilities after propagating through the Sun, $\bar{P}_{e \alpha}^{\odot}$, is very CPU-intensive due to the extent of the matter and neutrino distributions in the Sun. This subsection lists the necessary inputs and gives technical details on the manipulation of the outputs.

\section{Inputs}

The SSM provides the main two necessary quantities to calculate $\bar{P}_{e \alpha}^{\odot}$ with the effect of matter. First the electron density as a function of radius in the Sun, $n_{e}(r)$, was needed to calculate the matter potential $A_{\mathrm{CC}}$. The tabulated densities are shown on a log-scale in Figure 7.1a as a function of $r / R_{\odot}$, where $R_{\odot}=6.961 \times 10^{5} \mathrm{~km}$ is the radius of the Sun. On that scale the density has a roughly linear profile that can be parameterized, but, for precision, linear interpolation was used to read the data from the table without a significant increase in computing time [120].

Second, the radial profiles of the $\nu_{e}$ production region in the Sun, $w_{\nu_{i}}(r)$, were needed to account for the distribution of the neutrinos. Figure 7.1b shows the origin 


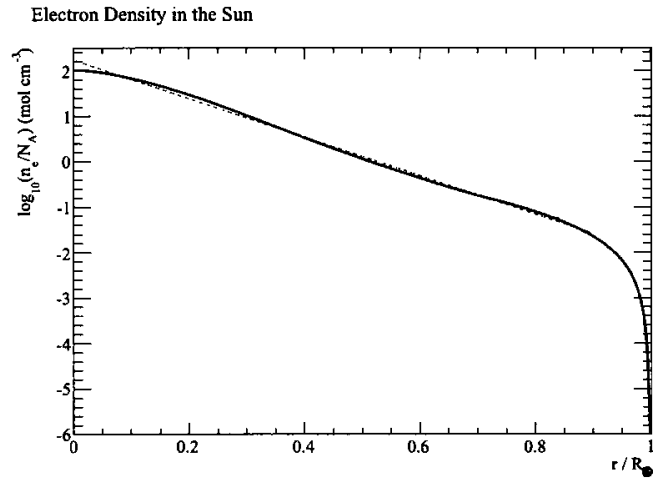

(a) Electron density.

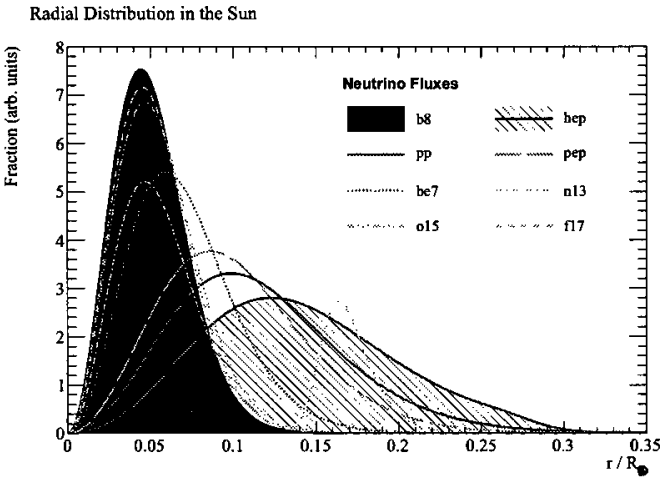

(b) Production regions.

Figure 7.1: (color) Radial profiles of the electron density and neutrino production as a function of position in the Sun, with $R_{\odot}=6.961 \times 10^{5} \mathrm{~km}$. Data obtained from the BS05(OP) model [147].

of the $\nu_{e}$ 's as a function of $r / R_{\odot}$ for each neutrino type. Particularly interesting for $\mathrm{SNO}$, the $\nu\left({ }^{8} \mathrm{~B}\right)$ and $\nu($ hep $)$ profiles peak at radii that are almost $0.1 R_{\odot}$ apart. All $\nu_{e}$ 's are produced within the Sun core with $r<0.35 R_{\odot}$.

Although the SSM is updated almost yearly, the above two fundamental inputs rarely suffer from significant changes from one calculation of the SSM to the other. Hence the Sun survival probabilities obtained with the BS05(OP) inputs also comply with more recent versions of the SSM.

\section{Outputs}

The tabulated $\bar{P}_{e \alpha}^{\odot}\left(\nu_{i}\right)$ were obtained after averaging the results of many $\bar{P}_{e \alpha}^{\odot}(k, z)$ calculations, each of them with a different starting point $(k, z)$ inside the Sun core. In total there were $N_{\text {pos }}=81$ points with radial, $r_{k}$, and angular, $z$, positions with respect to the Sun-Earth axis such that averages could be calculated for each neutrino 
type by applying the corresponding weights $w_{\nu_{i}}\left(r_{k}\right)$ :

$$
\bar{P}_{e \alpha}^{\odot}\left(\nu_{i}\right)=\sum_{k, z}^{N_{\text {pos }}} w_{\nu_{i}}\left(r_{k}\right) \bar{P}_{e \alpha}^{\odot}(k, z) / \sum_{k, z}^{N_{\text {pos }}} w_{\nu_{i}}\left(r_{k}\right)
$$

The weights $w_{\nu_{i}}\left(r_{k}\right)$ only depend on $k$ thus the angular positions were effectively averaged for each radial position. The 81 starting points are sufficient to sample the electron density and neutrino radial profiles and simulate the distributed nature of the origin of the neutrinos. Differences of at most $10^{-3}$ were observed by increasing the average to 200 points in certain regions of the space [126].

As explained in Section 7.1, the $2 \nu$ model only required the values of $\bar{P}_{e e}^{\odot}\left(\nu_{i}\right)$, and the $3 \nu$ model necessitated both $\bar{P}_{e e}^{\odot}\left(\nu_{i}\right)$ and $\bar{P}_{e \mu}^{\odot}\left(\nu_{i}\right)$. The $3 \nu$ quantities were obtained from the same calculation by changing the flavor $\alpha$ in Equation (7.12). The sum of the resulting phase-averaged probabilities fulfilled the conservation rule of Equation (1.10), with a negligible $0.2 \%$ spread over the whole oscillation parameter space.

\subsubsection{Propagation in the Earth}

The calculation of the probabilities in the Earth, $P_{\mathrm{E} 1}^{\oplus}$ and $P_{\mathrm{E} 2}^{\oplus}$, is less intensive CPUwise than the Sun calculation, but is complicated by the multiple paths sampled by the various exposure functions of each experiment.

\section{Inputs}

The matter density inside the Earth was taken from the Preliminary Reference Earth Model (PREM) [148, 149], which is the most widely accepted data since the density 
profile is inferred from seismological considerations. Figure 7.2 shows the discontinuities in the density profile as a function of position in the Earth, $r / R_{\oplus}$, where $R_{\oplus}=6371 \mathrm{~km}$ is the radius of the Earth. The standard chemical composition entering the conversion of matter to electron densities is a simple two-layer model where the ratio of protons to nucleons changes from $Z / A=0.468$ to 0.497 [150] at a radius of $3480 \mathrm{~km}$, where the core/mantle interface is located.

For the specific case of the SNO detector, the crust layer of the PREM table was slightly modified to reflect the higher density in the overburden norite rock above the detector and in the vicinity of Sudbury. The density values were assumed to be $2.90 \mathrm{~g} / \mathrm{cm}^{3}$ at the detector, $2.092 \mathrm{~km}$ below the surface, and $2.80 \mathrm{~g} / \mathrm{cm}^{3}$ at the surface [34]. Figure 7.2b emphasizes the modified region near $r / R_{\oplus}=1$. These small differences, averaged over all paths through the Earth, changed the probabilities calculated at the SNO detector by less than $0.1 \%$.

The livetime distribution of each experiment, $\eta^{\mathrm{d}}\left(\theta_{z}\right)$, was also required to weight the probabilities calculated from each neutrino path parameterized by the zenith angle $\theta_{z}$. The distributions were measured directly from the data run lists in the case of SNO and geometrically determined based on the detector coordinates for other experiments, assuming spherical symmetry in the Earth matter profile. The SNO livetime functions are shown in Figure 7.3 for the three phases of the experiment. The functions are rather similar. Typically, more neutrino data was taken during the night (with nadir angle $<90^{\circ}$ ) as a consequence of performing more detector diagnostics, calibrations, and repairs during the day. The geometrical calculation, corresponding to a one-year exposure period, is also shown for SNO using the coordinates $46^{\circ} 28^{\prime} 30^{\prime \prime}$ north latitude, $81^{\circ} 12^{\prime} 04^{\prime \prime}$ west longitude. 


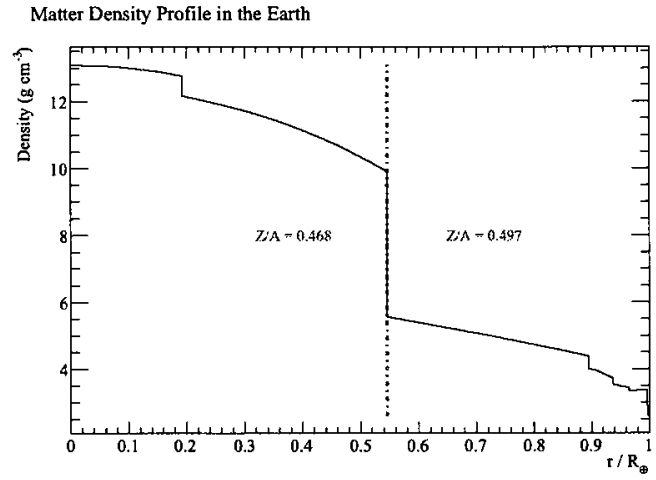

(a) All data.

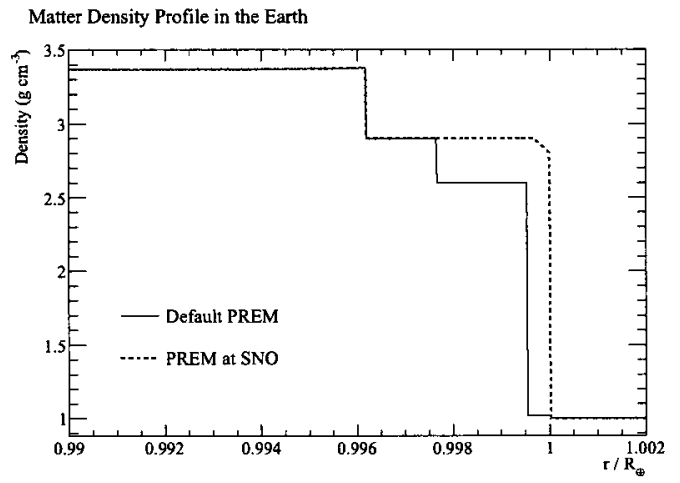

(b) Data near the surface.

Figure 7.2: Profile of the matter density inside the Earth, with $R_{\oplus}=6371 \mathrm{~km}$ [148]. Electron densities are obtained with $Z / A=0.468$ for $r / R_{\oplus}<0.546$ and $Z / A=0.497$ elsewhere.

\section{Outputs}

The tabulated $P_{\mathrm{E} 1}^{\oplus}(\mathrm{d})$ and $P_{\mathrm{E} 2}^{\oplus}(\mathrm{d})$ were obtained from many calculations, $P_{\mathrm{E} 1}^{(z, \mathrm{~d})}$ and $P_{\mathrm{E} 2}^{(z, \mathrm{~d})}$, starting from various Earth entrance points $z$ to the detector d, that were weighted by the experimental or calculated livetime $\eta^{\mathrm{d}}\left(\theta_{z}\right)$ :

$$
\begin{aligned}
& P_{\mathrm{E} 1}^{\oplus}(\mathrm{d})=\sum_{z}^{N_{\text {zen }}} \eta^{\mathrm{d}}\left(\theta_{z}\right) P_{\mathrm{E} 1}^{(z, \mathrm{~d})} / \sum_{z}^{N_{\text {zen }}} \eta^{\mathrm{d}}\left(\theta_{z}\right) \\
& P_{\mathrm{E} 2}^{\oplus}(\mathrm{d})=\sum_{z}^{N_{\text {zen }}} \eta^{\mathrm{d}}\left(\theta_{z}\right) P_{\mathrm{E} 2}^{(z, \mathrm{~d})} / \sum_{z}^{N_{\text {zen }}} \eta^{\mathrm{d}}\left(\theta_{z}\right)
\end{aligned}
$$

where $N_{\text {zen }}=90$ points, one for each $2^{\circ}$ in zenith angle. The Earth probabilities differ from one detector to another because of the livetime function and detector location, but, unlike their Sun counterparts, are independent of the neutrino type.

Additionally, the probabilities were consistently separated into a day/night fashion as in Chapter 6. Therefore two sets of tables were produced for each detector: one for the day, $P_{\mathrm{E} 1}^{\oplus}(\mathrm{d}, \mathrm{D})$ and $P_{\mathrm{E} 2}^{\oplus}(\mathrm{d}, \mathrm{D})$, that was almost undisturbed since the amount 


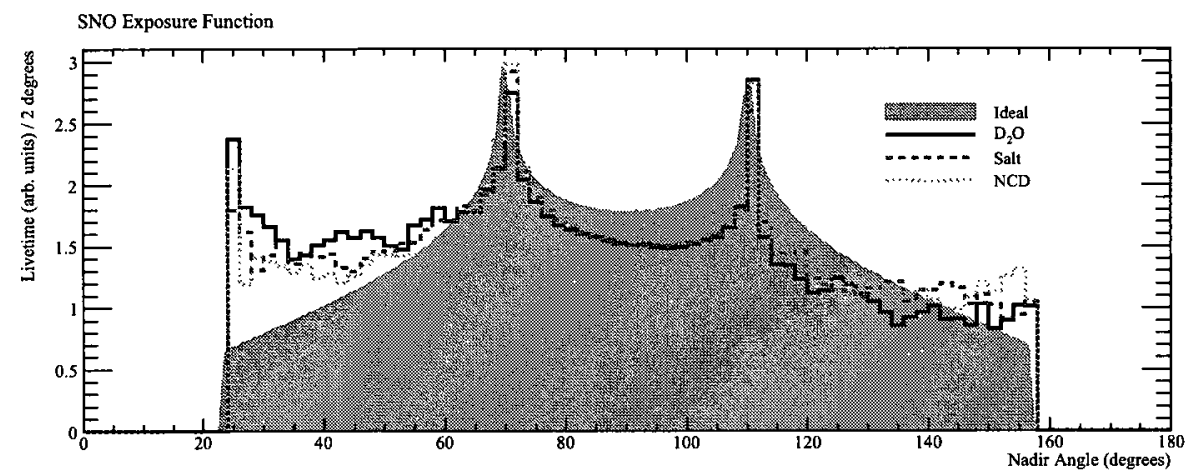

Figure 7.3: Experimental livetime distributions for the three SNO phases. The error in each $2^{\circ}$-bin is negligible. The ideal calculation corresponds to continuous exposure over a year of operation. The nadir angle is $180^{\circ}$ minus the zenith angle. A nadir angle larger (smaller) than $90^{\circ}$ corresponds to day (night) time.

of Earth matter above the underground detectors is quasi nonexistent during the day time, and the other for the night, $P_{\mathrm{E} 1}^{\oplus}(\mathrm{d}, \mathrm{N})$ and $P_{\mathrm{E} 2}^{\oplus}(\mathrm{d}, \mathrm{N})$, which was affected by longer paths through the dense layers of the Earth.

\subsubsection{Effects of the Third Mass Eigenstate}

The survival probabilities $P_{2 \nu}^{\mathrm{d}}$ and $P_{3 \nu}^{\mathrm{d}}$ were calculated for the parameter ranges in Table 7.1 using the outputs of the Sun and Earth calculations and formulae of Equations (7.8) and (7.10), respectively. For all values of $\tan ^{2} \theta_{12}$ and $\Delta m_{21}^{2}$ the calculations gave the same numerical result in the limit $\sin ^{2} \theta_{13} \rightarrow 0$.

One of the goals of this thesis is to extract the oscillation parameters but more specifically to constrain the mixing angle $\theta_{13}$ with the solar neutrino data. This subsection presents comparisons of $P_{3 \nu}^{\mathrm{d}}$ with $P_{2 \nu}^{\mathrm{d}}$ as a function of $\sin ^{2} \theta_{13}$ and $\Delta m_{31}^{2}$ to quantify the effects of the third mass eigenstate $\nu_{3}$ on the survival probability. The differences are highlighted in two typical regions of the standard two-parameter plane, that are of interest for SNO because of the large mixing angle $\theta_{12}$. The large mixing an- 
gle (LMA) region is defined from $\tan ^{2} \theta_{12} \in\left[10^{-1}, 1\right]$ and $\Delta m_{21}^{2} \in\left[10^{-5}, 10^{-3}\right] \mathrm{eV}^{2}$, and the low $\Delta m_{21}^{2}$ (LOW) region from $\tan ^{2} \theta_{12} \in\left[10^{-2}, 10\right]$ and $\Delta m_{21}^{2} \in\left[10^{-8}, 10^{-6}\right] \mathrm{eV}^{2}$.

\section{Effects of $\theta_{13}$}

Figure 7.4 shows the effects of $\sin ^{2} \theta_{13}$ on the survival probabilities as a function of $E_{\nu}$ in the LMA and LOW regions. The probabilities were averaged for equal contributions of day and night components at the SNO detector for clarity. Figures 7.4a and 7.4b show the absolute probabilities for the two regions, while Figures $7.4 \mathrm{c}$ and $7.4 \mathrm{~d}$ show the relative difference with respect to the $2 \nu$ calculation, in percent. The colored lines display the gradient of increasing values in $\sin ^{2} \theta_{13} \in\left[10^{-5}, 0.25\right]$, from Table 7.1. The comparisons show a rather weak shape distortion of $P_{e e}$, except for $E_{\nu}<6 \mathrm{MeV}$. The main effect is the decrease of the absolute scale in $P_{3 \nu}^{\mathrm{d}}$ with $\sin ^{2} \theta_{13}$, reaching a $25 \%$ shift at $10 \mathrm{MeV}$ at $\sin ^{2} \theta_{13}=0.25$ in the LMA and LOW regions.

The separate day/night curves were examined in a similar way. The main effect was a decrease of the difference $P_{3 \nu}^{\mathrm{d}}(\mathrm{N})-P_{3 \nu}^{\mathrm{d}}(\mathrm{D})$ for $E_{\nu}>10 \mathrm{MeV}$ with the increase of $\sin ^{2} \theta_{13}$, without noticeable effects at low energy. This increases the chances of observing a non-zero value of $\sin ^{2} \theta_{13}$ since no significant day/night asymmetry was observed in the survival probability curves. Therefore the precision on the survival probability scale obtained in Chapter 6 should be able to constrain the value of $\sin ^{2} \theta_{13}$.

\section{Effects of $\Delta m_{31}^{2}$}

The introduction of $\nu_{3}$ also requires values for $\Delta m_{31}^{2}$. Since $\Delta m_{31}^{2}$ appears in the form $\Delta m_{31}^{2} / E_{\nu}$ in Equation (1.11), it was set to a single value at $10 \mathrm{MeV}$ based on the 


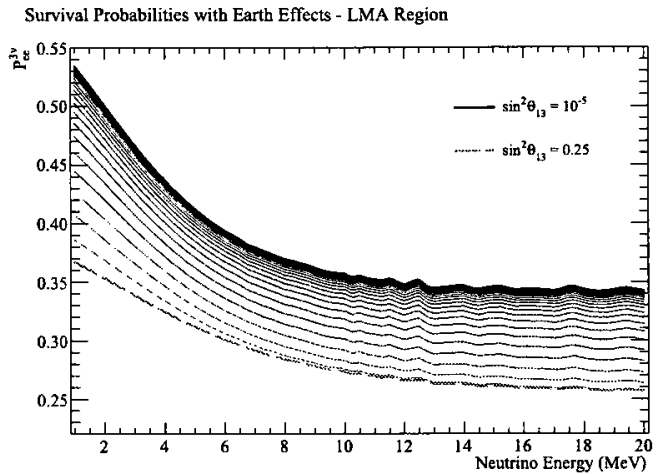

(a) $P_{3 \nu}^{\mathrm{d}}$ in the LMA region.

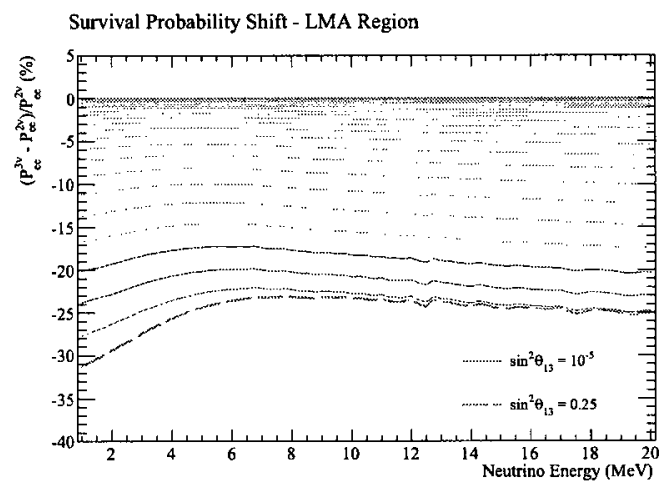

(c) $\left(P_{3 \nu}^{\mathrm{d}}-P_{2 \nu}^{\mathrm{d}}\right) / P_{2 \nu}^{\mathrm{d}}$ in the LMA region.

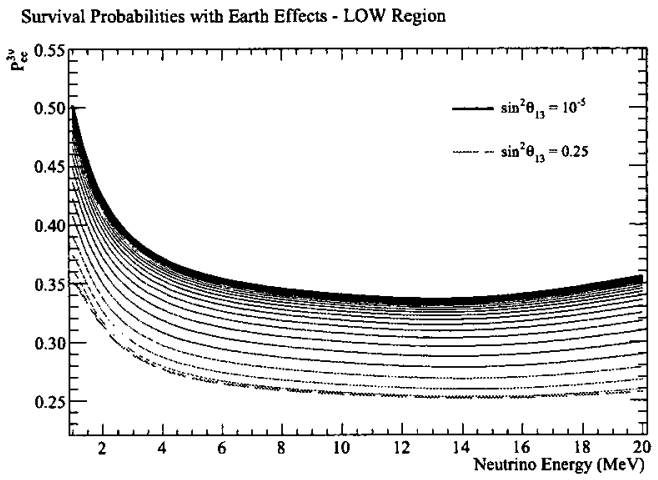

(b) $P_{3 \nu}^{\mathrm{d}}$ in the LOW region.

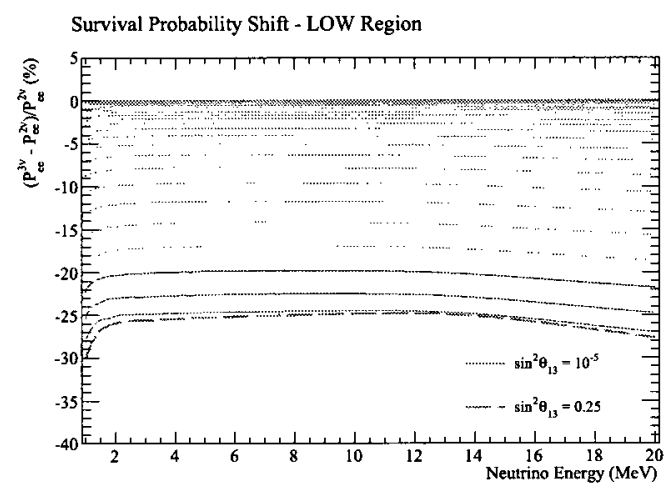

(d) $\left(P_{3 \nu}^{\mathrm{d}}-P_{2 \nu}^{\mathrm{d}}\right) / P_{2 \nu}^{\mathrm{d}}$ in the LOW region.

Figure 7.4: (color) Effects of $\theta_{13}$ on the $3 \nu$ survival probabilities. The survival probabilities are obtained at the SNO detector with parameters (a) $\tan ^{2} \theta_{12}=0.47$ and $\Delta m_{21}^{2}=7.6 \times$ $10^{-5} \mathrm{eV}^{2}$, (b) $\tan ^{2} \theta_{12}=0.47$ and $\Delta m_{21}^{2}=2 \times 10^{-7} \mathrm{eV}^{2}$. The relative differences in percent between $P_{3 \nu}^{\mathrm{d}}$ and $P_{2 \nu}^{\mathrm{d}}$ are shown for the (c) LMA and (d) LOW regions.

assumption that it has the smallest effect of all four parameters and that the amplitude variations in the oscillations are dominated by $\Delta m_{21}^{2}$. Assuming $\Delta m_{31}^{2} \simeq \Delta m_{32}^{2}$, the order of magnitude of the best current value $\Delta m_{32}^{2}=(2.55 \pm 0.45) \times 10^{-3} \mathrm{eV}^{2}[2]$ was used to set $\Delta m_{31}^{2} / E_{\nu}=10^{-4} \mathrm{eV}^{2} \mathrm{MeV}^{-1}$. Therefore the effective value of $\Delta m_{31}^{2}$ was slightly different for each neutrino energy in the range $E_{\nu} \in[0,20] \mathrm{MeV}$.

The phase-average technique mentioned in Subsection 7.1.1 is subject to residual effects due to $\Delta m_{31}^{2}$. The tabulated values of $P_{3 \nu}^{\mathrm{d}}$ were compared to a separate cal- 


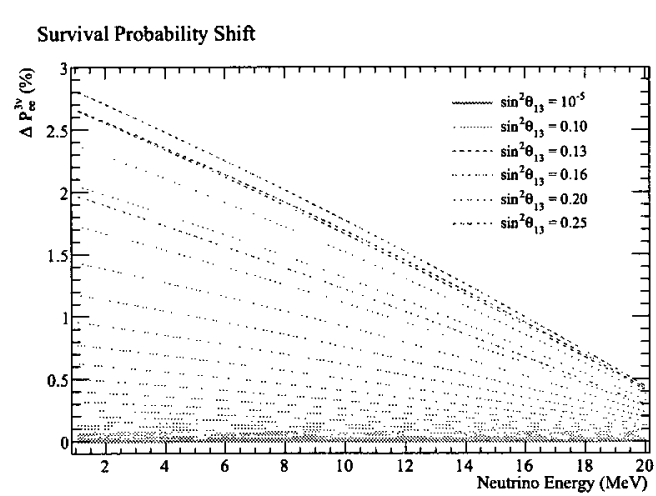

(a) Effect of $\Delta m_{31}^{2} / E_{\nu}$.

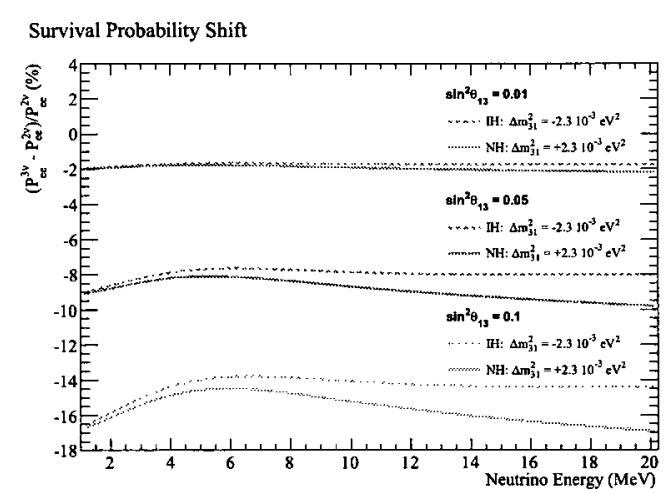

(b) Effect of $\operatorname{Sign}\left(\Delta m_{31}^{2}\right)$.

Figure 7.5: (color) Effects of $\Delta m_{31}^{2}$ on the $3 \nu$ survival probabilities. The survival probabilities are obtained before entering the Earth, with parameters $\tan ^{2} \theta_{12}=0.47$ and $\Delta m_{21}^{2}=7.6 \times 10^{-5} \mathrm{eV}^{2}$. (a) The difference of $\Delta m_{31}^{2} / E_{\nu}=10^{-4} \mathrm{eV}^{2} \mathrm{MeV}^{-1}$ relative to $\Delta m_{31}^{2}=2.3 \times 10^{-3} \mathrm{eV}^{2}$ is shown. (b) The difference relative to the $2 \nu$ calculation is shown.

culation with the value $\Delta m_{31}^{2}=2.3 \times 10^{-3} \mathrm{eV}^{2}$ consistently applied to all neutrino energies. Figure 7.5a shows the effect in the LMA region; the result is a systematic shift increasing as a function of $\sin ^{2} \theta_{13}$ with maximum amplitude of $\sim 3 \%$ at low energy. Although a $2-3 \%$ effect in $P_{3 \nu}^{\mathrm{d}}$ is not expected to affect the dominant parameters noticeably, an additional systematic uncertainty was included in the oscillation analysis to account for the unknown value of $\Delta m_{31}^{2}$.

The sign of $\Delta m_{31}^{2}$ cannot be determined by the current terrestrial and atmospheric neutrino oscillation experiments since matter effects in those experiments have not been yet observed. Figure $7.5 \mathrm{~b}$ shows, for interest, the effect of changing the sign of $\Delta m_{31}^{2}$, comparing the curves for normal ( $\mathrm{NH}$, positive) and inverted ( $\mathrm{H}$, negative) hierarchies. The shifts of $P_{3 \nu}^{\mathrm{d}}$ with respect to $P_{2 \nu}^{\mathrm{d}}$ are shown for three selected values of $\sin ^{2} \theta_{13}$. The result is a splitting effect of the differences at high energy, and the increase of the splitting with $\sin ^{2} \theta_{13}$. Thus the sign of $\Delta m_{31}^{2}$ could mimic the 
day/night effect at high energy despite the opposite effect due to $\sin ^{2} \theta_{13}$. Hence future analyses should probe the effects of $\Delta m_{31}^{2}$ further and perhaps try to distinguish between the two neutrino mass hierarchy scenarios with solar neutrino data.

\subsection{Parameter Constraints}

This section presents the confidence intervals of the neutrino oscillation parameters based on the SNO NC, CC and ES rates extracted in Chapter 5 and on data from other neutrino oscillation experiments. The oscillation parameter extraction is in all aspects identical to the survival probability extraction presented in Chapter 6, the only difference being that the input survival probabilities were calculated from $2 \nu$ or $3 \nu$ oscillation models instead of being found directly by a minimizer in $E_{\nu}$-space.

Two techniques were employed to obtain the parameter best-estimates and uncertainties. The first method is a grid-scan (GS) of the oscillation parameters, where the $\chi^{2}$ function of Equation (6.6) was evaluated and minimized with respect to the parameters $f_{i}$ at each point in space. The entire space, defined in Table 7.1, was scanned and the $\chi^{2}$ values stored as a function of the oscillation parameters. The $\chi^{2}$ differences, $\Delta \chi^{2}=\chi^{2}-\chi_{\min }^{2}$, listed in Table 7.2, were the indicators of the confidence levels (CL) in one- and two-dimensional space. Contour isolines were drawn with the $2 \mathrm{D}$ values; uncertainties were evaluated from the projection of the $\chi^{2}$ on each $1 \mathrm{D}$-axis. For all projections shown in this section, the $\chi^{2}$ was always minimized with respect to the undisplayed parameters at each point in space.

The second method is the minimization (MN) of the $\chi^{2}$ with TMinuit, where several iterations are performed to find the best survival probability curve and the corresponding oscillation parameters from the tables. The number of evaluations of 


\begin{tabular}{|cc|cc|}
\hline \hline$N \sigma$ & $\mathrm{CL}$ & $\Delta \chi_{1 \mathrm{D}}^{2}$ & $\Delta \chi_{2 \mathrm{D}}^{2}$ \\
\hline 1 & $68.30 \%$ & 1.000 & 2.298 \\
2 & $90.00 \%$ & 2.706 & 4.605 \\
3 & $95.00 \%$ & 3.841 & 5.991 \\
4 & $99.00 \%$ & 6.635 & 9.210 \\
5 & $99.73 \%$ & 9.000 & 11.83 \\
\hline \hline
\end{tabular}

Table 7.2: Number of standard deviations $(N \sigma)$ and confidence levels (CL) associated with the differences $\Delta \chi^{2}=\chi^{2}-\chi_{\min }^{2}$ in one and two dimensions. The values are obtained using two-sided intervals [101].

the $\chi^{2}$ function required to find the best-fit point and evaluate the asymmetric errors is in general smaller than in the GS method, but the method is sensitive to local minima. Therefore the MN method mainly served as a secondary check of the uncertainties on the oscillation parameters by constraining the fit around the minimum found with the GS method.

The above methods were utilized to extract the oscillation parameters from SNO in Subsection 7.3.1, all solar neutrino experiments in Subsection 7.3.2, and with the addition of the KamLAND experiment [42] in Subsection 7.3.3.

\subsubsection{Results from SNO}

The SNO-LETA rates were treated as in Subsection 6.3.1. Eight survival probability functions apply to SNO: $P_{\mathrm{D}_{2} \mathrm{O}}^{\mathrm{SNO}}\left({ }^{8} \mathrm{~B}, \mathrm{D}\right), P_{\mathrm{Salt}}^{\mathrm{SNO}}\left({ }^{8} \mathrm{~B}, \mathrm{D}\right), P_{\mathrm{D}_{2} \mathrm{O}}^{\mathrm{SNO}}\left(\right.$ hep, D), $P_{\text {Salt }}^{\mathrm{SNO}}($ hep, D), and their night-time duplicates. The $2 \nu$ and $3 \nu$ survival probabilities were scanned, the rates $F_{n}^{\text {th }}$ predicted, and the $\chi^{2}$ tables built in each case from the LETA correlation matrix and systematic uncertainties.

Figure 7.6 shows the projection of the $\chi^{2}$ in the $\left(\tan ^{2} \theta_{12}, \Delta m_{21}^{2}\right)$ plane, with 
selected 2D CL regions from Table 7.2. Figure 7.6a demonstrates how powerful the SNO-LETA rates are at constraining the allowed joint-space of the two main solar mixing parameters. The scan was first performed in low resolution with a coarse step size, therefore the position of the minima in Figure 7.6a are not accurate. The LMA and LOW regions nevertheless remain in the $2 \nu$-model, and $\theta_{13}$ enlarges, as expected, the confidence regions in the $3 \nu$-model. Significant improvements of the $2 \nu$-contours were achieved with SNO-LETA when comparing to the latest SNO contours in [27].

The minima were accurately found in the LMA region of the 2D plane after performing the GS with better resolution, with shorter step sizes and assuming linear interpolation between the $P_{e e}$-functions. Although the LOW region is also compatible with the SNO-LETA rates, Figure $7.6 \mathrm{~b}$ concentrates on the LMA region. The minima were $\chi_{\min }^{2(2 \nu)}=\chi_{\min }^{2(3 \nu)}=70.1$ with both the GS and MN methods. The $2 \nu$ parameters were $\tan ^{2} \theta_{12}=0.457_{-0.036}^{0.052}, \Delta m_{21}^{2}=6.31_{-2.10}^{2.40} \times 10^{-5} \mathrm{eV}^{2}$, and $f_{8 \mathrm{~B}}=0.916 \pm 0.031$. The $3 \nu$-model minimum gave the same $\tan ^{2} \theta_{12}$ and $\Delta m_{21}^{2}$, with worse uncertainties, due to the additional degree of freedom $\sin ^{2} \theta_{13}$. The parameters were $\tan ^{2} \theta_{12}=0.457{ }_{-0.036}^{+0.480}, \Delta m_{21}^{2}=6.31_{-3.86}^{+3.65} \times 10^{-5} \mathrm{eV}^{2}$, with the same value and uncertainty for $f_{8 \mathrm{~B}}$. The value of $\sin ^{2} \theta_{13}$ was $10^{-3}$ but could not be constrained on either sides of the allowed interval.

\section{Discussion and Interpretation}

The relatively large $\chi^{2} / \mathrm{ndf}=70.1 / 54$ obtained is a consequence of the constrained nature of the survival probabilities. Most LMA-type curves rise at low energy, as shown in Figure 7.4a, and are limited by unitarity to $P^{\mathrm{d}} \in[0,1]$. Thus one finds a small discrepancy coming from the low $T_{\text {eff }}$ part of the SNO-LETA spectra as depicted 


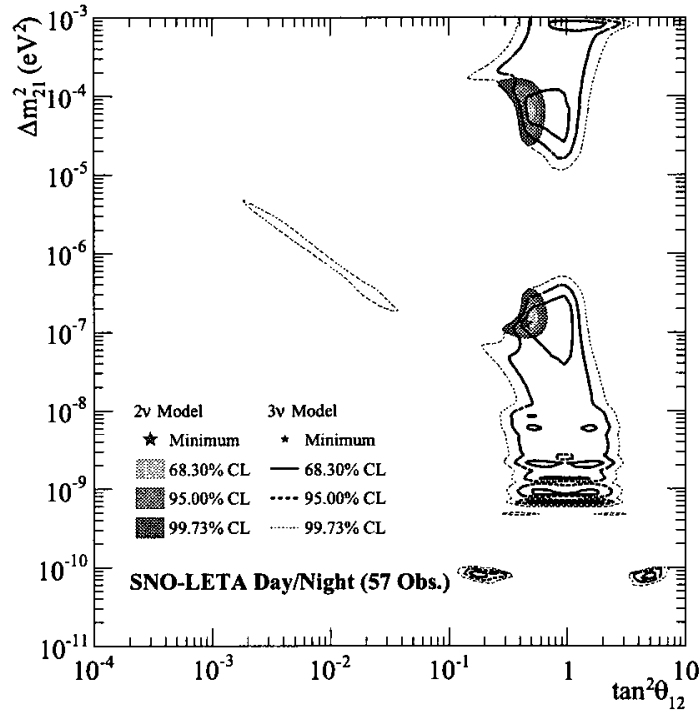

(a) Full space.

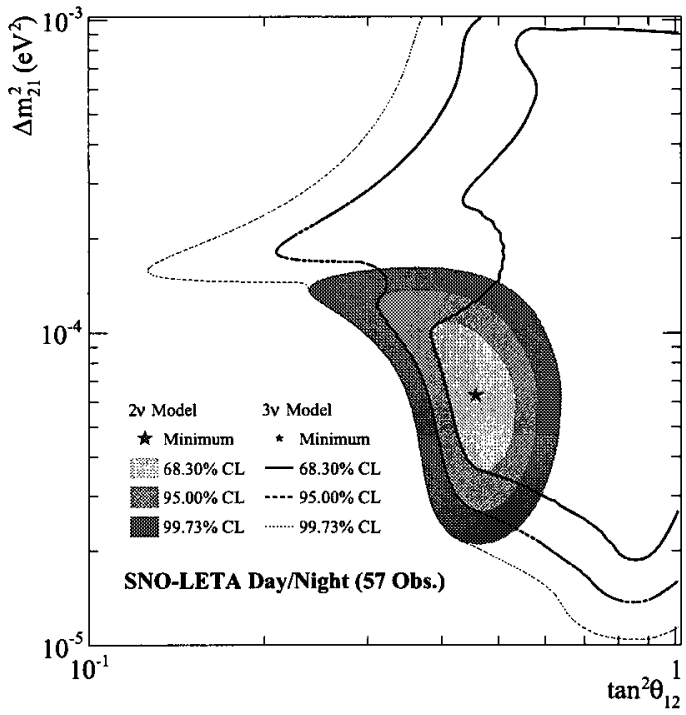

(b) LMA region.

Figure 7.6: Confidence regions of the oscillation parameters obtained with SNO-LETA in the $\left(\tan ^{2} \theta_{12}, \Delta m_{21}^{2}\right)$ space. The $\chi^{2}$ was minimized with respect to the undisplayed parameters. (a) In low resolution, the minimum is in the LOW region for the $2 \nu$-model and in the vacuum regime for the $3 \nu$-model. (b) In high resolution, the minimum is at $\tan ^{2} \theta_{12}=0.457$ and $\Delta m_{21}^{2}=6.31 \times 10^{-5} \mathrm{eV}^{2}$, in the LMA region, for both models.

in Figure 5.3. Although the most sensitive range in $E_{\nu}$ agrees well with typical LMA curves, the latter cannot be bent for a given set of mixing parameters, unlike the free-varying polynomials of Chapter 6 , to agree with all spectral bins in $T_{\text {eff }}$.

Nevertheless Figure 7.6b shows that the SNO-LETA rates set a barrier at $\Delta m_{21}^{2} \lesssim$ $10^{-4} \mathrm{eV}^{2}$ and $\tan ^{2} \theta_{12} \sim 0.4$, and that values $\Delta m_{21}^{2} \lesssim 10^{-5} \mathrm{eV}^{2}$ are prohibited by the observed smallness of the day/night asymmetry. The $\tan ^{2} \theta_{12}$ and $\Delta m_{21}^{2}$ parameters are well determined in both the $2 \nu$ and $3 \nu$ models, but $\sin ^{2} \theta_{13}$ could not be constrained with a compelling confidence level.

The failure of the SNO-LETA rates to constrain $\theta_{13}$ is not a surprising result. The effective data that test the survival probabilities are the $P_{e e^{-s c a l e}}$ via the $\mathrm{CC} / \mathrm{NC}$ 
ratio, and the flux normalization $f_{8_{\mathrm{B}}}$ via the $\mathrm{NC}$ integral rate. Hence in the $3 \nu$ model $\theta_{12}$ and $\theta_{13}$ simultaneously affect the $P_{e e^{-}}$scale, which results in the increase of the uncertainty on $\theta_{12}$ due to the weak sensitivity to $\theta_{13}$ at high $E_{\nu}$. This problem of the mixing angle correlation is solved by adding the rates of neutrino oscillation experiments that are sensitive to LE $\nu$ 's. Subsection 7.3 .2 gives the results of the oscillation analysis performed with all solar neutrino experiments.

\subsubsection{Results from Solar Neutrino Experiments}

The SNO and other solar rates were treated as in Subsection 6.3.2, where the list of rates is given in Table C.1 of Appendix C. In addition to the eight day/night survival probability tables for SNO-LETA, there are two averaged tables for SNO-NCD, and 32 more for other experiments, for a grand total of 42 tables.

Since the neutrino types are not degenerate, the $f_{i}$ parameters that affect LE experiments were not all allowed to vary in the fit as they were in Subsection 6.3.2. Although their scales were fixed to one, their uncertainties and cross-correlations were enforced with the SSM systematic uncertainties. The 20 uncertainties, provided as partial derivatives, are detailed in Section C.2.2 of Appendix C. The partial derivatives affecting $\nu\left({ }^{8} \mathrm{~B}\right)$ 's and $\nu($ hep)'s were removed since the SNO and SK data constrain the HE rates well.

Figure 7.7 shows the $2 \nu$ and $3 \nu$ confidence regions in the $\left(\tan ^{2} \theta_{12}, \Delta m_{21}^{2}\right)$ and $\left(\tan ^{2} \theta_{12}, \sin ^{2} \theta_{13}\right)$ space obtained from the 140 solar neutrino rates inputted to the GS method. Figure 7.7a compares the $2 \nu$ and $3 \nu$ regions of the combined solar fit. The $2 \nu$ parameters were $\tan ^{2} \theta_{12}=0.436_{-0.023}^{+0.041}, \Delta m_{21}^{2}=5.89_{-2.09}^{+1.75} \times 10^{-5} \mathrm{eV}^{2}$ at $\chi_{\min }^{2(2 \nu)}=133.13$. The inclusion of $\theta_{13}$ stretched the allowed regions of both $\tan ^{2} \theta_{12}$ and 


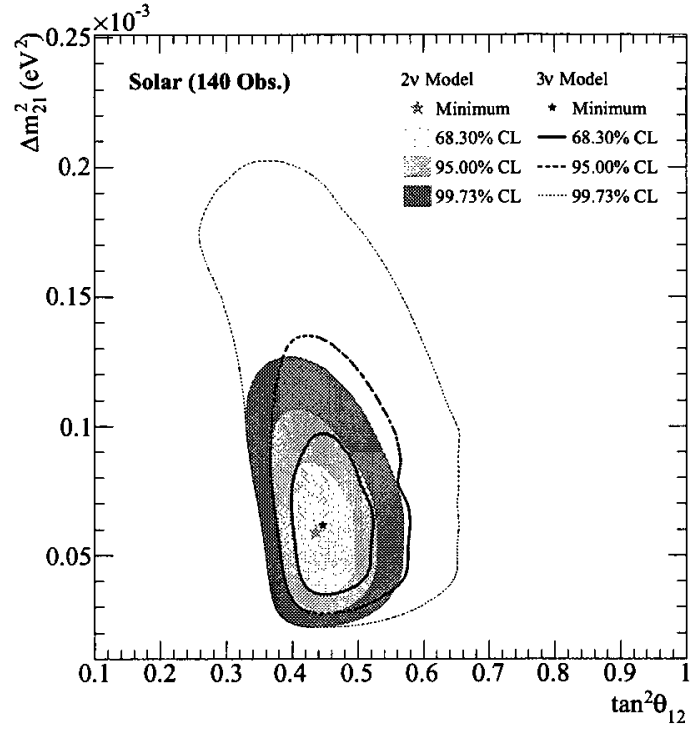

(a) Solar $2 \nu$ and $3 \nu$ models.

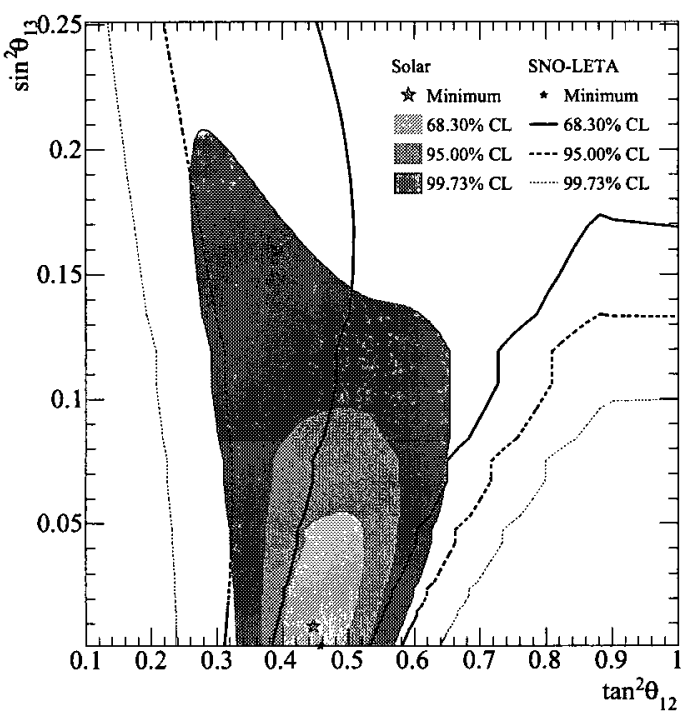

(b) Solar and SNO-LETA $3 \nu$ models.

Figure 7.7: Confidence regions of the oscillation parameters obtained with solar experiments in (a) the $\left(\tan ^{2} \theta_{12}, \Delta m_{21}^{2}\right)$ and (b) the $\left(\tan ^{2} \theta_{12}, \sin ^{2} \theta_{13}\right)$ space in the LMA region. The $\chi^{2}$ was minimized with respect to the undisplayed parameters.

$\Delta m_{21}^{2}$ towards larger values. The $3 \nu$-minimum was slightly different, corresponding to $\tan ^{2} \theta_{12}=0.447 \stackrel{{ }_{0}^{0.052}}{0.050}, \Delta m_{21}^{2}=6.17_{-2.28}^{+1.90} \times 10^{-5} \mathrm{eV}^{2}$. The shift was caused by a non-zero value of $\sin ^{2} \theta_{13}=8.9 \times 10^{-3}$ at $\chi_{\min }^{2(3 \nu)}=133.04$, which is however compatible with zero considering its uncertainty.

Figure $7.7 \mathrm{~b}$ compares the $3 \nu$ confidence regions obtained from the solar and SNOLETA analyses in the $\left(\tan ^{2} \theta_{12}, \sin ^{2} \theta_{13}\right)$ space. The contours close at large values of $\sin ^{2} \theta_{13}$ with the inclusion of all solar neutrino data. This means an upper limit can be set and yielded $\sin ^{2} \theta_{13}<0.06$ at the $95 \%$ CL from the marginalized $\chi^{2}$ projection on $\sin ^{2} \theta_{13}$. 


\section{Discussion and Interpretation}

The $\chi^{2} / \mathrm{ndf}=133.04 / 137$ demonstrates that the discrepancy of the SNO-LETA rates at low energy was compensated by the addition of LE rates from other solar experiments. Similar to the effect on the survival probability fit in Figure $6.7 \mathrm{~b}$, the bridge between the SNO-LETA low energy bins and LE experiments was recovered in the combined fit. The strength of the solar neutrino data is the stringent constraint on the mixing angle $\theta_{12}$, where a $+11 \%,-7 \%(+9 \%,-5 \%)$ precision was reached in the $3 \nu(2 \nu)$ analysis. The contribution of the SNO-LETA rates mainly affects the lower bounds of these uncertainties.

The contributions of the LE and HE experiments are well illustrated in Figure 7.7b. The HE rates (mainly SNO) constrain well $\theta_{12}$ when $\theta_{13} \rightarrow 0$. The CL lines stay narrow and shift to larger values of $\theta_{12}$ as $\theta_{13}$ increases, until the lines widen apart past $\sin ^{2} \theta_{13}=0.1$. The LE rates however, pull the allowed space towards smaller values of $\theta_{12}$ as $\theta_{13}$ increases, which is seen in the extend of the $99.73 \%$ CL in the upper-left corner of the figure. This crossing feature of the LE and HE rates in the $\left(\theta_{12}, \theta_{13}\right)$ plane produces this overall closed confidence region that prefers small values of $\theta_{13}$.

The latter observed features in the $\left(\theta_{12}, \theta_{13}\right)$ plane are explained by the following approximate analytic survival probability expressions [151]:

$$
P_{e e}^{\mathrm{LE}} \simeq \cos ^{4} \theta_{13}\left(1-\sin ^{2} 2 \theta_{12}\left\langle\sin ^{2}\left(\frac{\Delta m_{21}^{2} L}{4 E_{\nu}}\right)\right\rangle\right),
$$


for very LE neutrinos going through matter or for all neutrinos in vacuum, while

$$
P_{e e}^{\mathrm{HE}} \simeq \cos ^{4} \theta_{13} \sin ^{2} \theta_{12}
$$

for HE neutrinos that are converted by matter-enhanced oscillation. In the solar analysis, the HE SNO data dominates the overall precision and results in a small but perceptible positive correlation between $\theta_{12}$ and $\theta_{13}$. It is therefore crucial to add information in the fit, particularly from independent experiments with precise determination of the LE or vacuum oscillations.

The KamLAND contours, shown with the solar contours in Figure 7.8, were obtained using the data described in Subsection 7.3.3. Figure 7.8a shows excellent agreement of the dominant oscillation parameters, including the effect of $\theta_{13}$. The $68.30 \%$ CL regions overlap, which was not the case in the $2 \nu$ analyses shown in $[27,42]$ and reproduced in Subsection 7.3.3. Figure 7.8b illustrates the correlation generated by Equation (7.14). The KamLAND data show the anti-correlation between $\theta_{12}$ and $\theta_{13}$ that is amplified due to the great precision on $\Delta m_{21}^{2}$. Hence additional gain on the

determination of $\sin ^{2} \theta_{13}$ is expected from a combined analysis of the solar neutrino experiments with KamLAND, as presented in Subsection 7.3.3.

\subsubsection{Results from the Global Fit}

There are a number of current and future terrestrial oscillation experiments that can constrain the oscillation parameters and refine the results that were obtained with the solar experiments. This subsection only concentrates on one of them, the reactor experiment KamLAND [42], which was primarily built to confirm the oscillation 


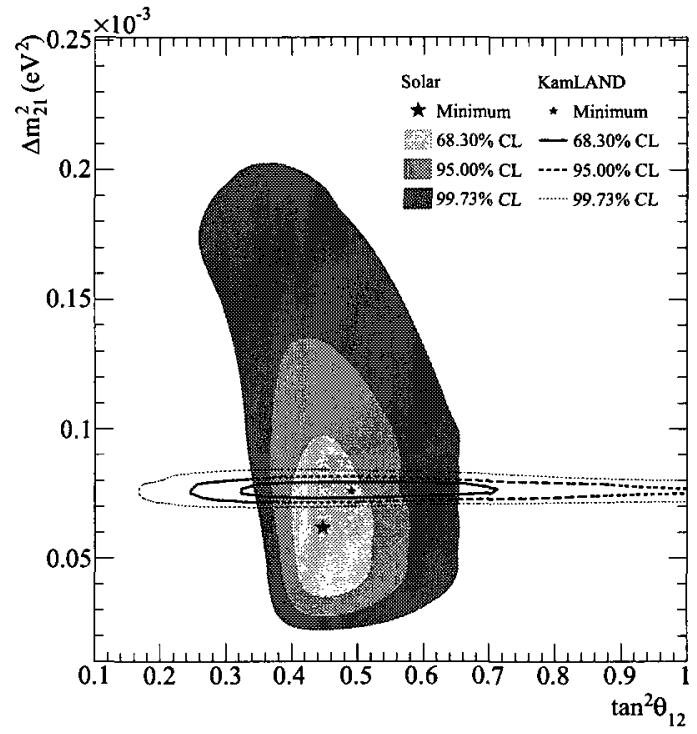

(a) Solar and KamLAND $3 \nu$ models.

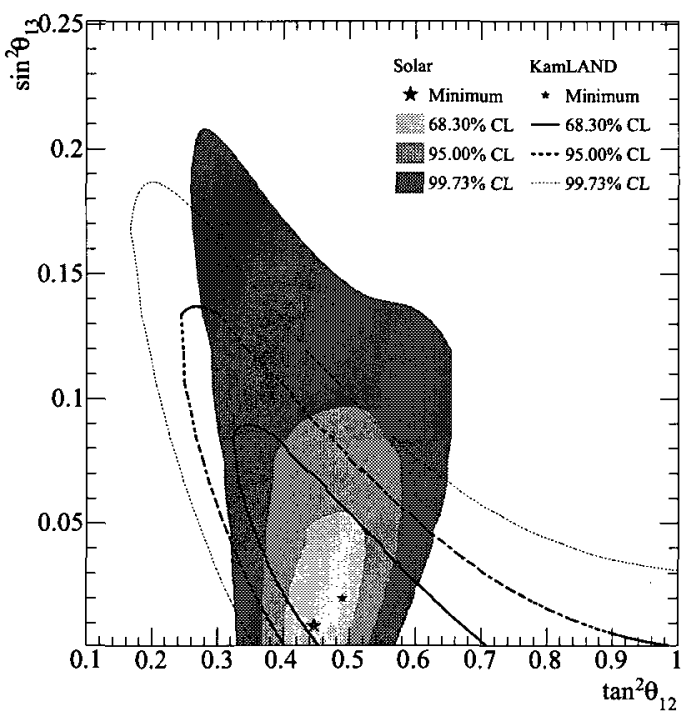

(b) Solar and KamLAND $3 \nu$ models.

Figure 7.8: Confidence regions of the oscillation parameters obtained with solar experiments and KamLAND in (a) the $\left(\tan ^{2} \theta_{12}, \Delta m_{21}^{2}\right)$ and (b) the $\left(\tan ^{2} \theta_{12}, \sin ^{2} \theta_{13}\right)$ space, in the LMA region. The $\chi^{2}$ was minimized with respect to the undisplayed parameters.

parameters measured by solar neutrino experiments. Since KamLAND is sensitive to anti-neutrinos coming from nuclear reactors, one must assume the solar $\nu$ 's and reactor $\bar{\nu}$ 's share the same oscillation parameters, as implied by CPT invariance, to perform a combined analysis.

The details of the treatment of the 16 KamLAND rates can be found in Subsection C.2.1 of Appendix C. The KamLAND and solar $\chi^{2}$ tables were added directly because the experiments have independent flux sources and systematic uncertainties. Therefore the respective $\chi^{2}$ values penalize each other such that the best common region of the oscillation parameter space remain. 


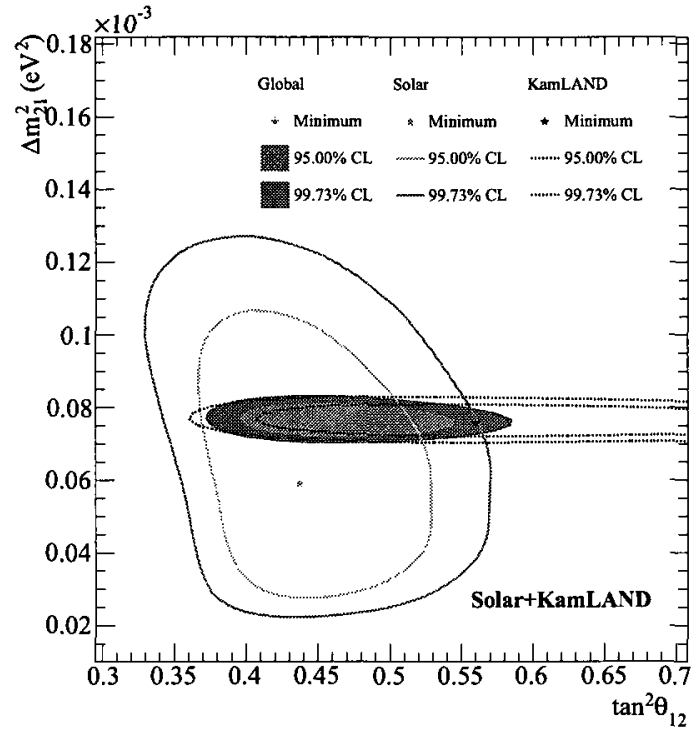

(a) Separate and combined regions.

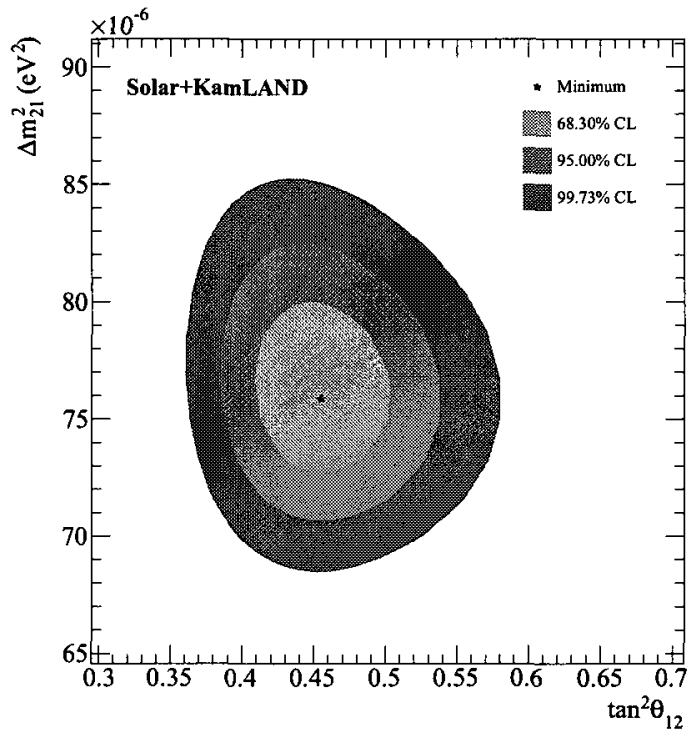

(b) Combined region.

Figure 7.9: Confidence regions of the oscillation parameters obtained with the combined fit of solar and KamLAND experiments in the LMA region for the $2 \nu$ model. The $\chi^{2}$ was minimized with respect to the undisplayed parameters. The minimum was $\chi^{2} / \mathrm{ndf}=$ $149 / 153$ at $\tan ^{2} \theta_{12}=0.457$ and $\Delta m_{21}^{2}=7.59 \times 10^{-5} \mathrm{eV}^{2}$.

\section{Two Neutrino Flavors}

Figure 7.9 shows the result of the global analysis performed with two neutrino flavors. The $2 \nu$-analysis gave a combined value of $\chi^{2} / \mathrm{ndf}=149 / 153$ with parameters $\tan ^{2} \theta_{12}=0.457 \underbrace{0.036}_{0.024}$ and $\Delta m_{21}^{2}=7.59_{-0.21}^{+0.21} \times 10^{-5} \mathrm{eV}^{2}$. Although the precision on $\Delta m_{21}^{2}$ is dominated by KamLAND, the precision on $\tan ^{2} \theta_{12}$ dropped to $+8 \%,-5 \%$, even if the best KamLAND value $\tan ^{2} \theta_{12}=0.56$ is significantly higher than the best solar value of $\tan ^{2} \theta_{12}=0.44$. Figure 7.9a shows the latter residual tension between the respective best-fit values of $\tan ^{2} \theta_{12}$, and Figure $7.9 \mathrm{~b}$ shows the resulting common confidence regions. Hence the $2 \nu$ parameters were: 


$$
\begin{gathered}
\theta_{12}=34.06_{-0.70}^{+1.05} \text { degrees } \\
\Delta m_{21}^{2}=7.59_{-0.21}^{0.21} \times 10^{-5} \mathrm{eV}^{2},
\end{gathered}
$$

where the reduction of $\sim 50 \%$ on the lower bound of the $\theta_{12}$ uncertainty compared to the results of $[27,42]$ is entirely due to the SNO-LETA rates.

\section{Three Neutrino Flavors}

Figure 7.10 shows the $3 \nu$ confidence regions in the $\left(\tan ^{2} \theta_{12}, \Delta m_{21}^{2}\right)$ and $\left(\tan ^{2} \theta_{12}\right.$, $\left.\sin ^{2} \theta_{13}\right)$ space obtained from the 140 solar neutrino and 16 KamLAND rates. The $3 \nu$-analysis gave a combined value of $\chi^{2} / \mathrm{ndf}=145.8 / 153$ with parameters $\tan ^{2} \theta_{12}=$ $0.45 \underbrace{+0.040}_{-0.025}, \Delta m_{21}^{2}=7.59_{-0.21}^{+0.23} \times 10^{-5} \mathrm{eV}^{2}$, and $\sin ^{2} \theta_{13}=2.51 \pm 1.29 \times 10^{-2}$. Although the precision on $\tan ^{2} \theta_{12}$ and $\Delta m_{21}^{2}$ is similar to the $2 \nu$-analysis, $\sin ^{2} \theta_{13}$ was found to be different than zero and constrained at the $1 \sigma$ level!

Figure 7.11 summarizes the $3 \nu$ constraints on the three dominant oscillation parameters, where the $99.73 \%(5 \sigma)$ and $68.30 \%(1 \sigma)$ levels are shown separately for the SNO-LETA, Solar, KamLAND, and Solar+KamLAND (Global) grid scans. The $1 \sigma$ errors of the global analysis were also checked with the MN method for comparison. Although the central values and errors were similar, limitations were encountered due to the coarse binning of the survival probability tables. Future analyses will need finer step sizes in all oscillation parameters to allow the MN method to get more precise errors.

One surprising feature arises in Figure 7.11f, where the global minimum for $\sin ^{2} \theta_{13}$ was found to be greater than the respective minima of the Solar and KamLAND analyses. This shift is explained by the correlations of $\theta_{13}$ with $\theta_{12}$. The global 


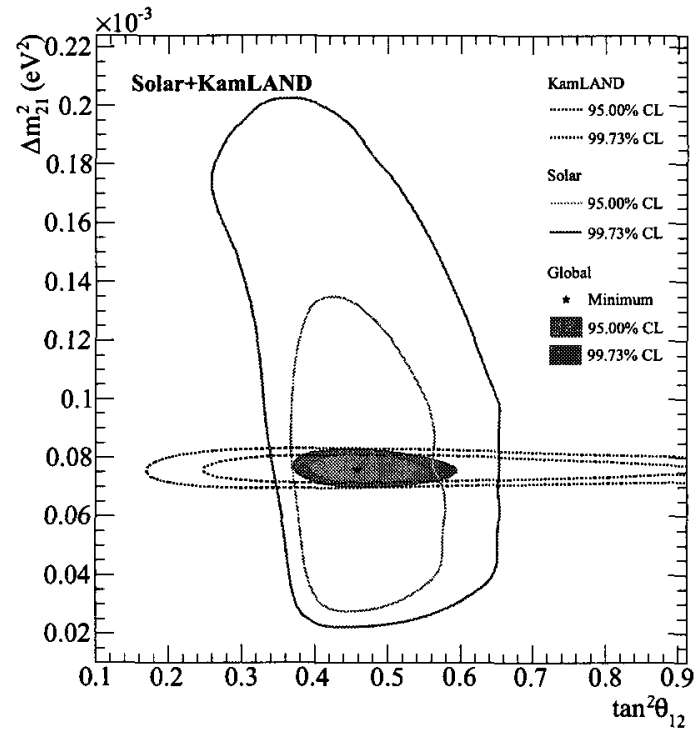

(a) Separate and combined regions.

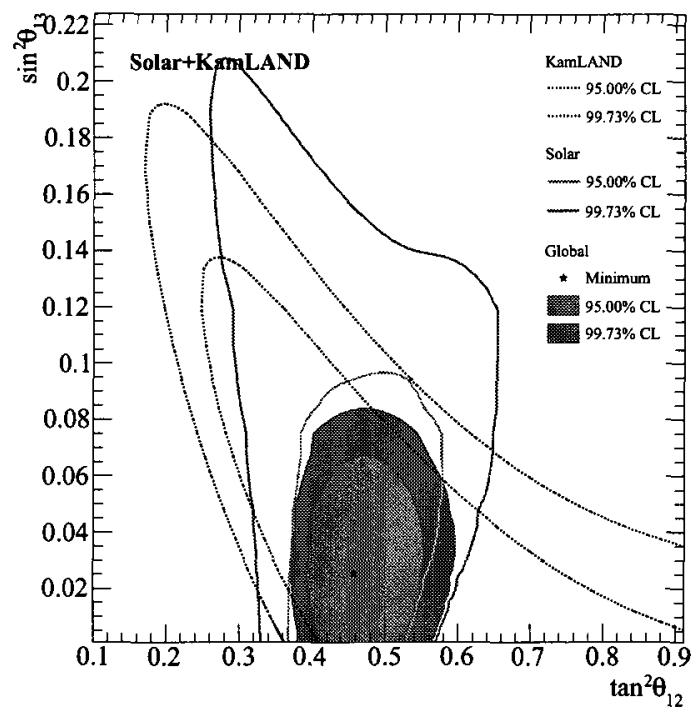

(c) Separate and combined regions.

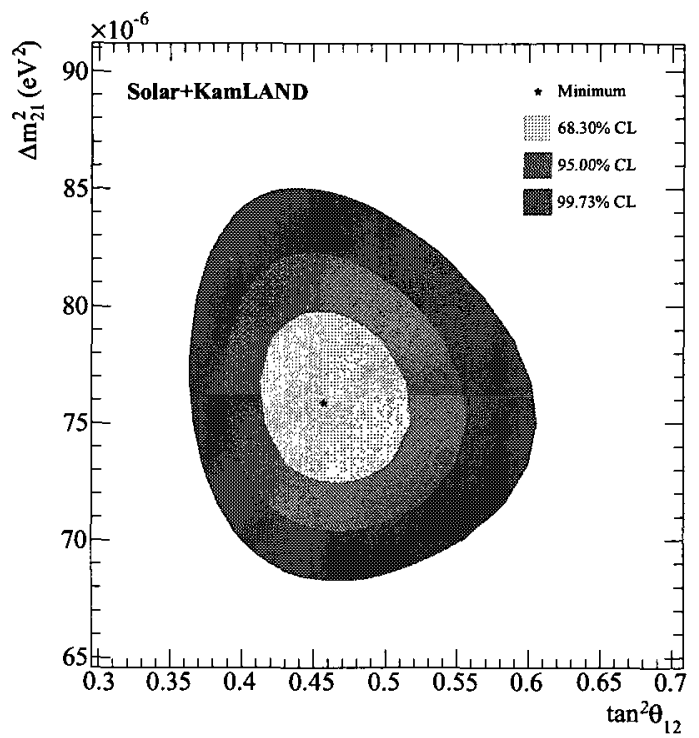

(b) Combined region.

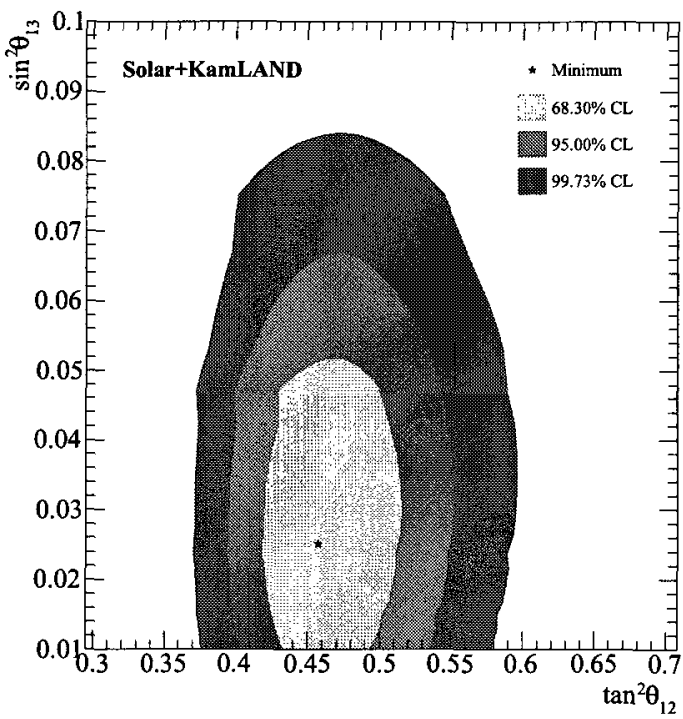

(d) Combined region.

Figure 7.10: Confidence regions of the oscillation parameters obtained with the combined fit of solar and KamLAND experiments in the LMA region for the $3 \nu$ model. The $\chi^{2}$ was minimized with respect to the undisplayed parameters. The minimum was $\chi^{2} / \mathrm{ndf}=$ $145.8 / 153$ at $\tan ^{2} \theta_{12}=0.457, \Delta m_{21}^{2}=7.59 \times 10^{-5} \mathrm{eV}^{2}$, and $\sin ^{2} \theta_{13}=0.025$. 
minimum in $\theta_{12}$ stands in between the Solar and KamLAND minima, as in Figure 7.9a, which simultaneously induces an increase in $\theta_{13}$ from solar (moving up-right) and KamLAND (moving up-left). Hence the residual discrepancy in $\theta_{12}$ between solar and KamLAND analyses is absorbed and is consistent with an overall larger common value of $\theta_{13}$.

Finally, the $3 \nu$ oscillation parameters were found to be:

$$
\begin{aligned}
\theta_{12} & =34.06_{-0.73}^{+1.16} \text { degrees } \\
\Delta m_{21}^{2} & =7.59_{-0.20}^{+0.23} \times 10^{-5} \mathrm{eV}^{2} \\
\theta_{13} & =9.1_{-2.4}^{+3.4} \text { degrees } .
\end{aligned}
$$

While the global analysis of Solar+KamLAND gives the world's best estimate of $\theta_{12}$, other experiments have set limits on $\theta_{13}$, such as CHOOZ [44] and SK-I with atmospheric neutrinos [152], that could potentially enhance the precision on $\theta_{13}$. The literature is rich in global analyses of all neutrino oscillation data. For example, see $[153,154]$.

\subsubsection{Summary}

The parameters relevant to the phenomenon of solar neutrino oscillation, $\tan ^{2} \theta_{12}$, $\Delta m_{21}^{2}$, and $\sin ^{2} \theta_{13}$, were extracted from the 140 solar $\nu$ - and 16 KamLAND $\bar{\nu}$-rates. The results, due to the improvement of the uncertainties in the LETA, were a better determination of $\tan ^{2} \theta_{12}$ and the first-time investigation of the effect of $\sin ^{2} \theta_{13}$.

Table 7.3 summarizes the measurements of the neutrino oscillation parameters given with the $1 \sigma$ errors evaluated from marginalized 1D-projections. The precision 


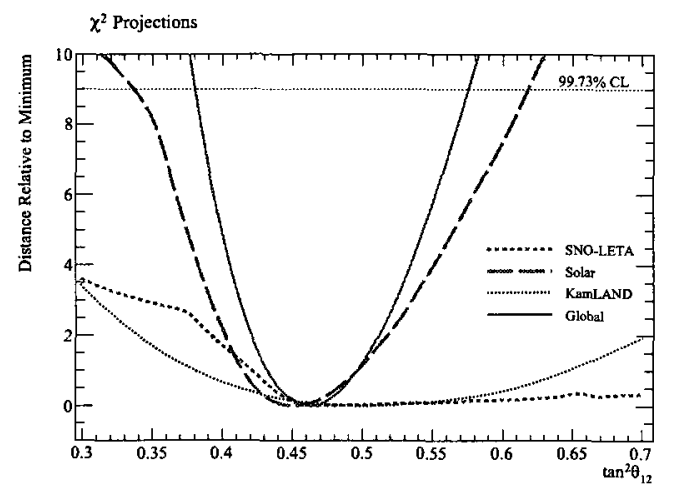

(a) $\Delta \chi^{2} \lesssim 10$ as a function of $\tan ^{2} \theta_{12}$.

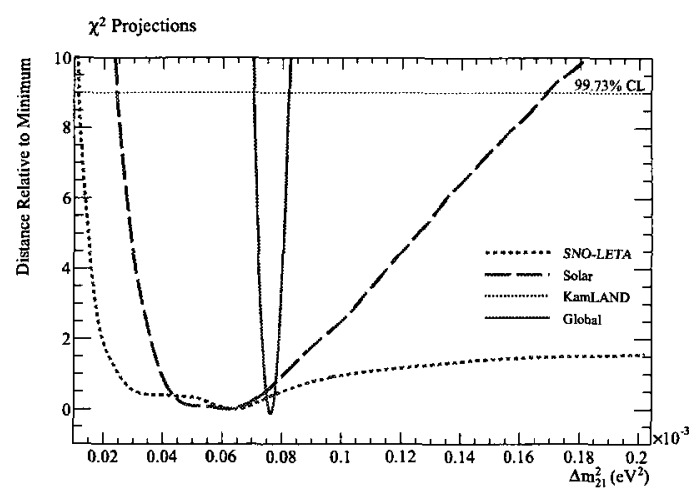

(c) $\Delta \chi^{2} \lesssim 10$ as a function of $\Delta m_{21}^{2}$.

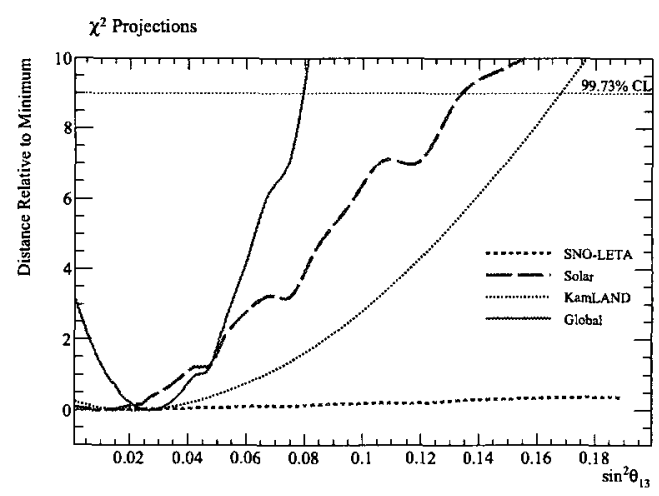

(e) $\Delta \chi^{2} \lesssim 10$ as a function of $\sin ^{2} \theta_{13}$.

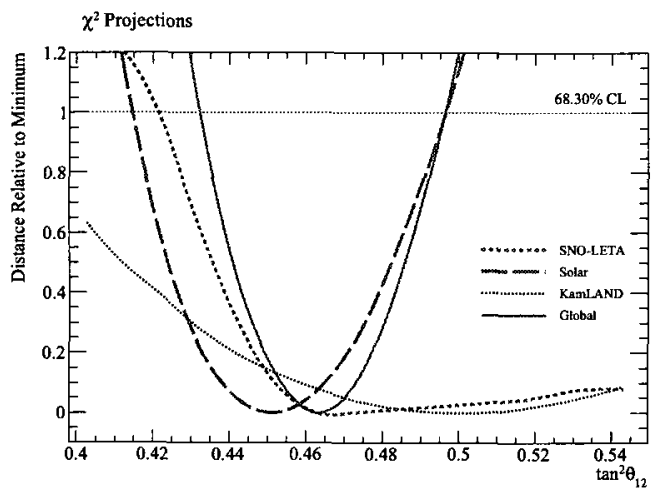

(b) $\Delta \chi^{2} \lesssim 1$ as a function of $\tan ^{2} \theta_{12}$.

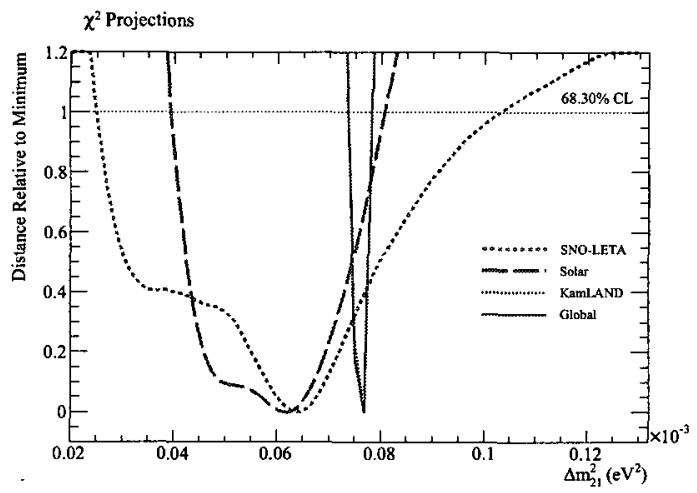

(d) $\Delta \chi^{2} \lesssim 1$ as a function of $\Delta m_{21}^{2}$.

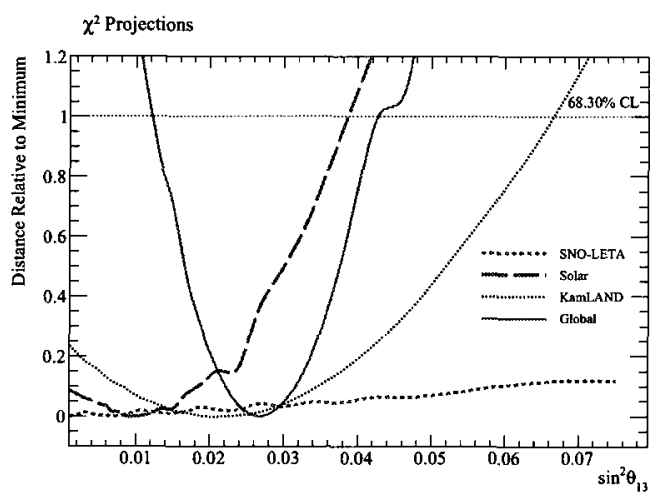

(f) $\Delta \chi^{2} \lesssim 1$ as a function of $\sin ^{2} \theta_{13}$.

Figure 7.11: Projections of the $\chi^{2}$-space onto each parameter axis for the $3 \nu$ analysis. The $\chi^{2}$ was minimized with respect to the undisplayed parameters. The differences $\Delta \chi^{2}=$ $\chi^{2}-\chi_{\min }^{2}$ are shown with two scales, corresponding to $5 \sigma(99.73 \% \mathrm{CL})$ on the left and $1 \sigma$ (68.3\% CL) on the right, for the $\tan ^{2} \theta_{12}, \Delta m_{21}^{2}$, and $\sin ^{2} \theta_{13}$ parameters, respectively. 


\begin{tabular}{|c|c|ccc|}
\hline \hline Model & Configuration & $\tan ^{2} \theta_{12}$ & $\begin{array}{c}\Delta m_{21}^{2} \\
\times 10^{-5} \mathrm{eV}^{2}\end{array}$ & $\begin{array}{c}\sin ^{2} \theta_{13} \\
\times 10^{-2}\end{array}$ \\
\hline \hline \multirow{3}{*}{$2 \nu$} & SNO-LETA & $0.457_{-0.036}^{+0.052}$ & $6.31_{-2.10}^{+2.40}$ & - \\
\cline { 2 - 5 } & Solar & $0.436_{-0.023}^{+0.041}$ & $5.89_{-2.09}^{+1.75}$ & - \\
\cline { 2 - 5 } & Solar+KamLAND & $0.457_{-0.024}^{+0.036}$ & $7.59_{-0.21}^{+0.21}$ & - \\
\hline \hline \multirow{3}{*}{$3 \nu$} & SNO-LETA & $0.457_{-0.036}^{+0.480}$ & $6.31_{-3.86}^{+3.65}$ & $0.10_{-0.1}^{+25.0}$ \\
\cline { 2 - 5 } & Solar & $0.447_{-0.032}^{+0.050}$ & $6.17_{-2.28}^{+1.90}$ & $0.89_{-1.36}^{+3.17}$ \\
\cline { 2 - 5 } & Solar+KamLAND & $0.457_{-0.025}^{+0.040}$ & $7.59_{-0.21}^{+0.23}$ & $2.51_{-1.29}^{+1.84}$ \\
\hline \hline
\end{tabular}

Table 7.3: Summary of the measurements of the neutrino oscillation parameters.

on $\tan ^{2} \theta_{12}$ is primarily due to the lower bound constrained by the SNO-LETA spectra, while the precision on $\Delta m_{21}^{2}$ parameter is dominated by KamLAND.

Finally the second-order effects of $\sin ^{2} \theta_{13}$ could be constrained in the global fit. Although the uncertainty was of the order of $60 \%$, the best-fit value was found to disagree with zero by about $2 \sigma$. Hence the current solar neutrino and terrestrial data are precise enough to be able to see a hint that the value of $\theta_{13}$ is small but not zero. Therefore, more information was provided on the structure of the MNSP neutrino mixing matrix that will perhaps guide the design of future neutrino experiments.

Based on the above interesting results, Chapter 8 concludes this thesis. by comparing the survival probabilities obtained in Chapters 6 and 7 , and discusses the prospects towards future precision in neutrino physics. 


\section{CHAPTER 8}

\section{Conclusion}

The quest for the understanding of neutrino properties, started nearly 80 years ago, has led today's physicists to a better knowledge of the neutral light leptons. Experimental and theoretical research groups from all around the world have contributed to broaden the search from the simple confirmation of the hypothesis of their existence to the development of detection techniques and the measurement of their fundamental properties. Although the questions of their role in the standard model of particle physics, their nature, and their masses are still unanswered, it is reasonable to predict that clear explanations will come in the near future, primarily due to the rich and beautiful variety of experiments that have been proposed by the scientific community.

One major contribution to neutrino and astroparticle physics was made by the Canadian SNO experiment with the discovery of solar neutrino oscillation, solving a long-standing problem of rate deficits observed by former neutrino detectors. Since this discovery, the SNO and solar neutrino experiments have turned into a collective precision program targeted at measuring the flux of all neutrino flavors, to validate the physics of the Sun, and the theoretical parameters at the source of neutrino flavor change. This thesis presented these classical solar neutrino physics measurements in Chapter 5,6 , and 7 , respectively. Undoubtedly these measurements will be refined in 
the future with new generations of multi-purpose experiments, probing the enormous fluxes of low energy neutrinos coming from the Sun.

\section{One Last Plot}

The survival probability extraction method presented in Chapter 6 is rather unconventional, but represents a novel alternative to present the solar neutrino spectral information. One important verification that must be made, in order to confirm the validity of the matter-enhanced oscillation hypothesis, is the compatibility of the weighted average of all relevant survival probabilities predicted by the oscillation model, at the LMA solution, with the model-independent average survival probability function. Figure 8.1 shows that comparison. The analytic fit function was imported from Figure $6.7 \mathrm{~b}$, while the LMA prediction was obtained by averaging the 42 probability tables at the minimum $\chi^{2}$ point, and using the $3 \mathrm{D}$ contours $(1 \sigma$ with $\left.\Delta \chi^{2}=3.53\right)$ in the three oscillation parameters $\tan ^{2} \theta_{12}, \Delta m_{21}^{2}$, and $\sin ^{2} \theta_{13}$ to obtain the model error band. The agreement is excellent, since the two $1 \sigma$-bands overlap, confirming matter-enhanced oscillation as a viable mechanism to explain not only the deficits of $\nu_{e}$ rates observed on Earth, but also the different behaviors of low and high energy neutrinos. The so-called MSW-effect with three neutrino flavors therefore yields a large dominating mixing angle, $\theta_{12} \sim 34^{\circ}$, a small mass difference squared, $\Delta m_{21}^{2} \sim 8 \times 10^{-5} \mathrm{eV}^{2}$, and possibly an additional tiny mixing component, $\theta_{13} \sim 9^{\circ}$. Hence the solar neutrino experiments have come a long way from questioning the SNP to confirming the physical mechanism at the origin of neutrino oscillation. Three of the eight oscillation parameters were constrained in this dissertation, which constitutes a great experimental outcome. 
Solar Neutrino Average Survival Probabilities

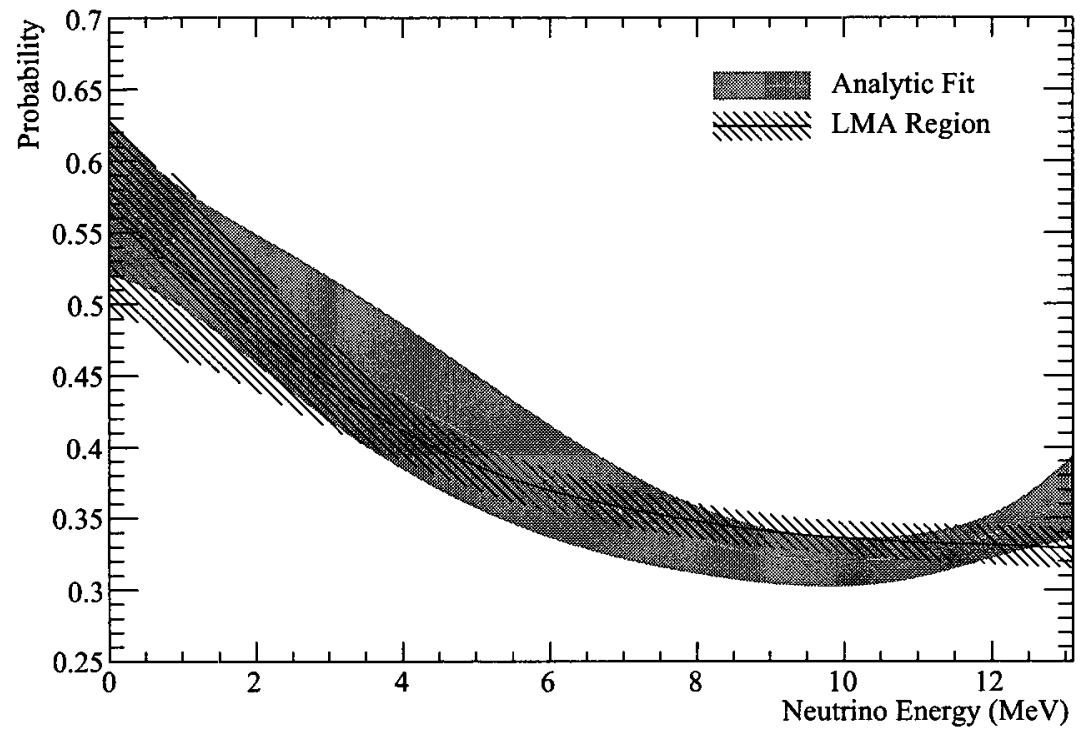

Figure 8.1: Survival probability functions extracted from solar neutrino data. The analytic fit is taken from Figure 6.7b and the LMA region curve was extracted from the parameter space in Figure 7.7.

\section{The Thesis Summary}

After the introduction of the general problem in solar neutrino physics in Chapter 1 - whether the flux normalization and survival probability could be measured simultaneously - the SNO experiment was described as the key to the SNP because of its multiple observation channels. The SNO detector, detailed in Chapter 2, could measure the NC, CC, and ES fluxes with three different configurations, employing independent means to measure the neutron rate produced by the neutrinos through the NC reaction.

The enhancements in the optical calibrations of SNO developed at the beginning of the third phase, described in Chapter 3, were employed in both the low energy threshold analysis of the first and second phases and in the first integral flux extraction with 
the third phase data. In the low energy threshold analysis, the calibration constants and Monte Carlo PMT models were refined, leading to a better agreement between the simulations and data, which indirectly affected the assessment and treatment of systematic uncertainties in the combined analysis.

These improvements also led, in the NCD phase, as demonstrated in Chapter 4, to an alternate procedure to characterize the optics of the detector. The method had an overall precision comparable to the previous phases, despite the complications introduced by the shadowing and reflection effects in the PMT data due to the NCD array. The benefits were the determination of the positions of the 40 NCDs and the confidence that shadows and reflections were not biasing either the optics, energy, or position reconstruction algorithms in the third phase of SNO.

The low energy threshold analysis was introduced and performed in Chapter 5. As a consequence of hard collaborative effort, the analysis results were the most precise determination of the SNO NC flux to date and will be published in the near future. The NC flux, in units of the solar standard model prediction, was $0.91(1.00 \pm 0.04)$, compared to $1.00 \pm 0.16$ in the $\mathrm{BS} 05(\mathrm{OP})$ model. Therefore the measured total neutrino production rate in the Sun agrees well with the prediction, resulting in high confidence in the accuracy of the complex model of stellar evolution.

The energy-unconstrained fit used to extract the signals allowed the day/night $\mathrm{CC}$ and ES spectra to be treated independently, enabling a further investigation of spectral distortions. These spectra were the inputs to the survival probability extraction method introduced in Chapter 6. The survival probabilities were obtained from the SNO and solar neutrino experiments using two different parameterizations in $E_{\nu}$-space. The resulting functions were smooth decreasing probabilities without 
measurable distortions due to Earth effects.

The survival probabilities were then calculated in Chapter 7 using the most accepted explanation of neutrino oscillation, the enhancement of resonant flavor transitions in the Sun in the presence of high matter densities. The model-predicted probabilities were then tested with the same experimental data to obtain precise estimates of the neutrino oscillation parameters. The three relevant parameters were extracted with solar data, and the combined analysis with the KamLAND anti-neutrino data improved the precision on the second mixing angle, in the form of $\sin ^{2} \theta_{13}$, which was found, for the first time, to be different than zero by two standard deviations. Although the latter angle was small, the effect was important enough to diminish the residual discrepancies between the solar neutrino experiments and KamLAND in the conventional two-flavor oscillation analysis.

The precise determination of the oscillation parameters in the solar neutrino sector, presented in this thesis, opens the door for future experiments to unveil the remaining unknown parameters, measure $\theta_{13}$ with increased precision, and constrain all the elements of the leptonic mixing matrix. With the current matrix parameterization, a non-zero value of $\theta_{13}$ might allow one to probe the effects of CP-violation in the lepton sector, towards the understanding of the role of neutrinos in weak interactions. In addition, the sign of the second mass difference squared, $\Delta m_{31}^{2}$, will clarify the hierarchy of the neutrino masses. And of course eventually, the small masses must also be determined. The hope is that the next generation of hard-working physicists will take over this work and narrow down the few remaining mysteries about neutrinos. And then, after the immense feeling of satisfaction, and perhaps a short vacation, they will move on to tackle dark matter and dark energy... 


\section{APPENDIX A}

\section{More on Optical Calibration}

This appendix contains additional information that complements the results of Chapters 3 and 4. Mainly, the PMT occupancy correction formulae are found in Section A.1 and the OCA systematic uncertainties are described in detail in Section A.2. The media attenuations are tabulated in Section A.3. The construction of the PMT response banks used by SNOMAN is explained in Section A.4.

\section{A.1 PMT Occupancy Corrections}

PMT occupancy corrections modify the raw number of counts measured by a PMT. This section explains the two formulae referred to in the analysis of Chapters 3 and 4: the multiple photo-electron (MPE) and combined corrections. The combined correction takes into account the contributions of MPE and NCD reflections (NCDRs).

The raw estimator of the $j^{\text {th }}$ PMT's occupancy in run $i$ is a quantity relative to the number of input laser pulses $N_{i}^{\text {pulses }}$ :

$$
O_{i j}^{\text {obs }}=\frac{N_{i j}^{\text {counts }}}{N_{i}^{\text {pulses }}}
$$

which, at the single PE level, is constrained to take values between zero and one because $N_{i j}^{\text {counts }}$ is the number of counts in the \pm 4 ns window which cannot exceed 
$N_{i}^{\text {pulses }}$. Thus, the occupancy $O_{i j}^{\text {obs }}$ is interpreted as the probability of detecting a signal.

\section{A.1.1 Multiple Photo-Electron Correction}

MPE effects arise in the occupancy when simultaneous pulses coming from many PEs are registered as single counts. The MPE correction therefore corrects for the lack of counts observed.

The correction is based on the Poisson probability of observing $n$ counts in PMT $j$ due to the true occupancy, $\mu_{i j}$, the mean of the Poisson distribution $P\left(\mu_{i j}, n\right)$. The probability of detecting a signal $O_{i j}^{\text {obs }}$ is one minus the probability of getting no signal:

$$
O_{i j}^{\text {obs }}=1-P\left(\mu_{i j}, 0\right)
$$

Thus solving for $\mu_{i j}$ gives the corrected PMT occupancy:

$$
\mu_{i j}=-\ln \left(1-O_{i j}^{\mathrm{obs}}\right)
$$

This operation effectively adds counts to the underestimated quantity $O_{i j}^{\text {obs }}$. This correction is applied to all PMT occupancies of all optical scans presented in this thesis, except in the NCD phase where an extra term is added to correct for NCD reflections (see Section A.1.2).

\section{Uncertainty of the MPE Correction}

The error propagation formula [101] is used to evaluate the statistical error due to the MPE correction. The formula assumes that the error on $N_{i}^{\text {pulses }}$ is very small and 
that the covariance between $N_{i}^{\text {pulses }}$ and $N_{i j}^{\text {counts }}$ is negligible:

$$
\Delta \mu_{i j}=-\sigma_{N_{j}^{\mathrm{c}}} \frac{\partial}{\partial N_{j}^{\mathrm{c}}}\left\{\ln \left(1-O_{i j}^{\mathrm{obs}}\right)\right\}=\frac{\sigma_{N_{j}^{\mathrm{c}}}}{N_{i}^{\mathrm{p}}-N_{j}^{\mathrm{c}}}
$$

A similar formula can be found when other terms are added to the correction formula, but in general Equation (A.4) gives the leading statistical component from $N_{i j}^{\text {counts }}$.

\section{A.1.2 NCD Reflection Correction}

This subsection details how the NCD reflection correction described in Section 4.4 is obtained. The intensity of the light reflected off the NCDs is estimated from the geometry and the relative positions of the LB, NCD array, and PMT. It is then used as input in the modified correction formula. The modified correction formula arises from the fact that the effects of NCD reflections in the occupancy of a PMT cannot be corrected alone without including the MPE effect that is always present. The NCD reflection part of the correction must be applied to decrease the PMT occupancy such that the overall MPE correction does not suffer from an increase that is too large. The next few paragraphs explain how the new terms are approximated.

\section{First Order Approximation}

The correction corresponds to the observed occupancy minus the expected contribution from the NCD reflections:

$$
\mu_{i j}^{\prime}=\mu_{i j}+\mu_{i j}^{\mathrm{ref}}=a_{i j}\left(1+w_{i j}\right)
$$


where $a_{i j}$ is a normalization term, proportional to the PMT acceptance, and $w_{i j}$ is the probability of NCD reflections hitting the PMT $j$ in run $i$. Defining $\xi \equiv w_{i j}$, the NCD reflection correction to $\mu_{i j}$ is the ratio of the observed to the true occupancy:

$$
O_{i j}^{\text {corr }}=\frac{\mu_{i j}^{\prime}}{\mu_{i j}}=1+\xi
$$

such that the corrected occupancy is

$$
\mu_{i j}=\frac{\mu_{i j}^{\prime}}{1+\xi} .
$$

The correction of Equation (A.7) can be applied after $\mu_{i j}^{\prime}$ is pre-corrected using Equation (A.2). However, it is desirable to obtain $\mu_{i j}$ directly from a combined occupancy correction. Since the NCD reflections are expected to contribute weakly to the overall number of photons, a term describing the probability of registering a count from a PE produced by NCD reflections, $P\left(\mu_{i j}, 1\right)$, is added to Equation (A.2):

$$
O_{i j}^{\mathrm{obs}}=1-P\left(\mu_{i j}, 0\right)+w_{i j} P\left(\mu_{i j}, 1\right)
$$

where $w_{i j}$ weights the probability of the NCD-reflected photons to hit the PMT face. Assuming the form of Equation (A.8), the verification that the correction corresponds to the ratio in Equation (A.7) is made by developing the Taylor series of $1-P\left(\mu_{i j}^{\prime}, 0\right)$ 
around $\xi=0$ at first order:

$$
\begin{aligned}
P\left(\mu_{i j}^{\prime}, 0\right) & =e^{-\mu_{i j}(1+\xi)} \\
& \approx e^{-\mu_{i j}}+\left.\frac{d}{d \xi} e^{-\mu_{i j}(1+\xi)}\right|_{\xi=0} \times \xi \\
& =e^{-\mu_{i j}}+\left.\xi e^{-\mu_{i j}} \frac{d}{d \xi} e^{-\mu_{i j} \xi}\right|_{\xi=0} \\
& =e^{-\mu_{i j}}-\mu_{i j} \xi e^{-\mu_{i j}}=e^{-\mu_{i j}}\left(1-\mu_{i j} \xi\right) .
\end{aligned}
$$

Comparing the expression for $1-P\left(\mu_{i j}^{\prime}, 0\right)$ obtained with this approximation and Equation (A.8), the result is equivalent:

$$
\begin{aligned}
1-P\left(\mu_{i j}^{\prime}, 0\right) & \approx 1-e^{-\mu_{i j}}\left(1-\mu_{i j} \xi\right)=1-e^{-\mu_{i j}}+e^{-\mu_{i j}} \mu_{i j} \xi \\
& =1-P\left(\mu_{i j}, 0\right)+\xi P\left(\mu_{i j}, 1\right) .
\end{aligned}
$$

Hence the correction factor $\xi$ must be used to weight the term $P\left(\mu_{i j}, 1\right)$ in the NCD reflection part of the PMT occupancy correction formula.

\section{NCD Reflection Probability}

To apply the combined correction in the form of Equation (A.8), the probability $w_{i j}$ must be estimated from the detector geometry. For a LB position $i$ with PMTs $j$ and NCDs $k$, the acceptance $A_{i j}$ of the PMTs to the reflected photons is defined as the sum of the products of the PMT and NCD solid angles, times a factor that takes into account the fraction of illuminated surface seen by the PMT, within a predefined time window $t_{w}$ :

$$
A_{i j}\left(t_{w}\right)=\sum_{k}^{N_{\mathrm{NCD}}} \Omega_{i k} \Omega_{k j} F_{k j}
$$


where the solid angle $\Omega_{i k}$ is relative to $4 \pi$, and $\Omega_{k j}$ to $2 \pi$. The time window value determines what fraction of a given NCD cylinder is used in the solid angle calculation given the different path lengths from the LB to the different sub-sections on the NCDs. The wider the time window, the larger the solid angles $\Omega_{i k}$ because reflections then can come from a larger surface on the NCDs and reach the PMTs. Because of the cylindrical symmetry of the NCDs, the fraction of illuminated NCD surface is a simple cosine distribution with an arbitrary maximum:

$$
F_{k j}=\frac{1}{2}(1+\cos \theta)
$$

where $\theta$ is $180^{\circ}$ minus the angle between the LB-NCD and NCD-PMT vectors, after they have been projected in the $x y$-plane. The $F_{k j}$ factor weights the probability of PMTs that are at the back of a NCD with respect to the LB to see the reflection due to the small fraction of illuminated surface available. Because the above assumption is such that the reflections are diffuse, the reflectivity is used to weight the acceptance $A_{i j}$ such that the reflection probability $G_{i j}$ is

$$
G_{i j}=R(\lambda) A_{i j}=R(\lambda) \times \sum_{k}^{N_{\mathrm{NCD}}} \Omega_{i k} \Omega_{k j} F_{k j} .
$$

The factor $G_{i j}$, when evaluating the probability of detecting reflected photons relative to regular photons, must be expressed as a fraction of the solid angle $\Omega_{i j}$ :

$$
\frac{G_{i j}}{\Omega_{i j}}=w_{i j}
$$




\section{Combined Correction of the PMT Occupancy}

Based on Equation (A.13) and the geometrical quantity $w_{i j}$, the occupancy correction formula is:

$$
O_{i j}^{\text {obs }}=1-P\left(\mu_{i j}, 0\right)+w_{i j} P\left(\mu_{i j}, 1\right)=1+\left(\mu_{i j} w_{i j}-1\right) \times e^{-\mu_{i j}}
$$

Equation (A.15) is defined as the combined MPE and NCDR occupancy correction formula, also Equation (4.6), and needs to be solved for each LB-PMT combination.

\section{A.2 Systematic Uncertainties in the NCD Phase}

This section outlines the systematic errors included in the total uncertainties quoted in Section 4.5. Table A.1 briefly describes the main classes of systematic effects. The existing, or pre-NCD, systematic errors, including the errors related to the source, first defined in [56], are reviewed in Subsection A.2.1. The systematic errors of the PMT efficiencies and detector asymmetries, introduced in Chapter 3, are covered in Subsection A.2.2. Lastly, the new effects such as shadows and reflections are discussed in Subsection A.2.3; a more detailed discussion on the systematic errors specific to the NCD phase can be found in $[97,155]$.

The systematic errors were evaluated by smearing observables or fixing parameters of the optical model in order to perform secondary fits. The effects were then quantified from the differences between the nominal and secondary shifted fits. The applied shifts were in most cases larger than the expected shifts, such that the effect of a given systematic error could be distinguished from statistical fluctuations. The 


\begin{tabular}{|l|c|l|l|c|}
\hline \hline Class/Origin & $i$ & Effect & Application & $f_{i}$ \\
\hline & 1 & Radial position scale & $R^{\prime}=R \times 1.01$ & 0.20 \\
Source position & 2 & Radial position shift & $R^{\prime}=R+5 \mathrm{~cm}$ & 0.20 \\
Subsection A.2.1 & 3 & Radial position smear & $R^{\prime}=R+\mathrm{N}(0,5 \mathrm{~cm})$ & 0.20 \\
& 5 & Source $z$-position & $Z^{\prime}=Z+5 \mathrm{~cm}$ & 0.40 \\
& 6 & Source $x$-position & $X^{\prime}=X+5 \mathrm{~cm}$ & 0.20 \\
& 7 & Amplitude & $d_{\text {PMT }}^{\prime}=d_{\mathrm{PMT}}-3 \mathrm{~cm}$ & 0.50 \\
\hline Source distribution & 8 & Uniformity & $L_{i j}^{\prime}=L_{i j}^{2}$ & 0.05 \\
Subsection A.2.1 & 9 & PMT variability & $L_{i j}^{\prime}=1$ & 0.05 \\
\hline Statistics & 10 & $\chi_{i j}^{\prime}=0$ & 0.20 \\
Subsection A.2.1 $(4 \sigma)$ & $\chi^{2}<16$ & 1.00 \\
& 11 & $\chi^{2}$ cut $(3 \sigma)$ & $\chi^{2}<9$ & 1.00 \\
\hline Method/Response & 12 & PMT efficiencies & $\epsilon_{j}^{\prime}=\epsilon_{j}+\mathrm{N}(0,10 \%)$ & 0.50 \\
Subsection A.2.2 & 13 & Response asymmetry & Selection in PMT $(z)$ & 0.50 \\
\hline NCD phase & 14 & Tolerance parameter & $\Delta L^{\prime}=2.5 \mathrm{~cm}$ & 0.50 \\
Subsection A.2.3 & 15 & NCD reflectivity & $R(\lambda)^{\prime}=0$ & 1.00 \\
& 16 & Reflection probability & $w_{i j}^{\prime}=w_{i j} \times 10$ & 0.20 \\
\hline \hline
\end{tabular}

Table A.1: Systematic uncertainties of the optical constants in the NCD phase. The errors are evaluated by identifying an effect, applying it through a shift in the fit, and then by weighting the shifts with the factors $f_{i}$. See text for details.

differences were then weighted with factors to adjust them to the true size of the shifts.

\section{A.2.1 Existing Systematic Errors}

The definitions of these systematic errors are the same as in [56]. They were consistently evaluated for all NCD-phase scans. Minor upgrades enforced that identical PMT subsets, before and after the virtual shifts, were compared. This was particularly important to prevent PMTs identified by the NCD shadow cut from amplifying the differences of other systematic effects. 


\section{Source Position}

The systematic errors due to the LB positions were obtained by applying virtual shifts to the LB coordinates by amounts corresponding to the total average uncertainty of $2 \mathrm{~cm}$. The observables impacted by such shifts were the distances in the three media $\left(d_{i j}^{\mathrm{d}, \mathrm{a}, \mathrm{h}}\right)$ and PMT solid angles $\left(\Omega_{i j}\right)$.

Radial Position Scale (1) : A radial scaling of $1 \%$ was applied to all LB positions to assess uncertainties on the detector size in time units; ECA, PCA, and assumed optical properties such as group velocities and wavelengths. This systematic affected the attenuations significantly, through an increase of $\Omega_{i j}$ as high as $2 \%$. The resulting changes in the fitted attenuations ranged between 20\% and 50\%. Applying the weighting factor $f_{1}=0.2$ to obtain an effective $0.2 \%$ shift, the observed change in the attenuations was about $10 \%$ for the $\mathrm{D}_{2} \mathrm{O}$ and $20 \%$ for the $\mathrm{H}_{2} \mathrm{O}$ attenuations. The change in PMTR was small, except at high angles where the refracted light paths were displaced by $2-5 \mathrm{~cm}$, smearing the incident angles and causing them to fall in a neighboring bin. The effect on the PMTR was less than $1 \%$ after the weighting factor was applied.

Radial Position Shift (2) : An absolute $5 \mathrm{~cm}$ shift was applied to all LB positions. Different from the previous systematic, this shift was the same in size for all runs. This affected the attenuations the most, where variations of at most $100 \%$ were seen. Because the $L B$ radial position uncertainty is symmetric with a size of $2 \mathrm{~cm}$, this effect was weighted by $f_{2}=0.2$ to bring it down to an effective $1 \mathrm{~cm}$ shift outwards. The induced changes were about $15 \%$ for the $\mathrm{D}_{2} \mathrm{O}$ and $25 \%$ for the $\mathrm{H}_{2} \mathrm{O}$ attenuations. 
As for the previous systematic, the PMTR was affected by less than $1 \%$ after the weighting factor was applied.

Radial Position Smear (3) : The third radial systematic effect was a random normal smearing of the radial distance by $5 \mathrm{~cm}$, similar to the previous, except that it allowed for negative shifts. Differences in the attenuations were small because most position shifts were smaller than $5 \mathrm{~cm}$, and because of accidental position-to-position shift cancellations in the parameters. The changes in the attenuations were nevertheless larger than the nominal statistical uncertainties. A weighting factor of $f_{3}=0.2$ was used for the same reasons as before, which resulted in an approximate $5 \%$ change in both the $\mathrm{D}_{2} \mathrm{O}$ and $\mathrm{H}_{2} \mathrm{O}$ attenuations. The change in the PMTR was within the statistical variation in each bin of incident angle.

Source $z$-Position (4): This was a positive shift of $5 \mathrm{~cm}$ of all $z$-coordinates, to take into account the $z$-shifts observed between the manipulator and the fitted LB positions and possible shifts between the detector coordinate systems. This shift primarily affected the $\mathrm{H}_{2} \mathrm{O}$ attenuations which increased by about $7 \%$ on average. The large effect on $\alpha_{\mathrm{h}}$ is a consequence of the limitations of the manipulator system, which allowed for more longer distances $d_{\mathrm{h}}^{i j}$ at the bottom of the AV. Since positions are shifted up, it leads to shorter paths in the AV and $\mathrm{H}_{2} \mathrm{O}$ and resulted in larger attenuation coefficients. This single coordinate shift was weighted with a factor of $f_{4}=0.4$ to bring back the $5 \mathrm{~cm}$ to $2 \mathrm{~cm}$. The effective uncertainty on the $\mathrm{H}_{2} \mathrm{O}$ attenuations was therefore closer to $2.5 \%$. The effect in the $\mathrm{D}_{2} \mathrm{O}$ attenuations and PMTR were negligible. 
Source $x$-Position (5) : This was a positive shift of $5 \mathrm{~cm}$ of the LB $x$-coordinates. A slightly smaller weighting factor of $f_{5}=0.2$ was applied to take into account the correlations with the previous shifts. The attenuations and PMTR did not vary by more than the statistical uncertainties. The fitted LB distribution $L_{i j}$ was influenced by such a shift, as the fit interpreted the shift as the source being brighter along the positive $x$-axis, but the changes were smaller than $1 \%$.

Source Size (6) : This shift accounted for the physical size of the LB ( $R_{\mathrm{LB}}=$ $5.45 \mathrm{~cm}$ ) by shortening the distances $d_{i j}$ by $3 \mathrm{~cm}$. Differences of the order of $15 \%$ in the attenuations were observed on average. Because the actual optical center of the $\mathrm{LB}$ cannot be resolved to better than $1 \mathrm{~cm}$, a conservative weighting factor of $f_{6}=0.5$ was assigned. The effect was therefore estimated to $8 \%$ on the $\mathrm{D}_{2} \mathrm{O}$ and $\mathrm{H}_{2} \mathrm{O}$ attenuations. The change in the PMTR was negligible.

\section{Source Distribution and Statistics}

Changes in the LB light intensity can affect the rest of the parameters. To evaluate this effect the $L_{i j}$ obtained with the nominal fit were altered and the fit repeated. The systematic errors associated with the fit statistics were studied by either removing the PMT variability $v_{i j}^{2}$ from the $\chi^{2}$ or by imposing more restrictive $\chi^{2}$ cuts.

LB Amplitude (7) and Uniformity (8): The effect of the amplitude was obtained by squaring the relative intensity therefore amplifying the residual differences in the nominal fit. The shifts observed in the $\mathrm{D}_{2} \mathrm{O}$ and $\mathrm{H}_{2} \mathrm{O}$ attenuations were smaller than $5 \%$, and the PMTR changes were negligible. Because the $L_{i j}$ function is part of the optical model, a factor of $f_{7}=0.05$ was used to weight down this effect already 
included in part in the statistical uncertainties. The effect of the uniformity was obtained by assuming the LB was ideal by setting the relative intensity to 1.0 (flat) in all directions. The effects were similar to the amplitude, and for the same reason a factor of $f_{8}=0.05$ was applied. Overall these LB effects were mostly negligible.

PMT Variability (9): This systematic error was obtained by simply removing the additional uncertainty $v_{i j}^{2}$ in the denominator of Equation (3.6). The result was a change in the individual PMT $\chi^{2}$ values. From Figure 3.4, the maximum $v_{i j}$ value for short wavelengths was about $15 \%$ (except at $620 \mathrm{~nm}$ where it could be as large as $35 \%$ ); the weighting factor was set to a value of $f_{9}=0.2$. The resulting shifts in the attenuations were about $5 \%$ and $75 \%$ for the $\mathrm{D}_{2} \mathrm{O}$ and $\mathrm{H}_{2} \mathrm{O}$, respectively, revealing the large coupling between the $\mathrm{H}_{2} \mathrm{O}$ attenuation and PMTR bins at large incident angles. The change in the PMTR was as large as $2 \%$. After the weighting factors were applied the contribution from this effect was about $1 \%(5 \%)$ for the $\mathrm{D}_{2} \mathrm{O}\left(\mathrm{H}_{2} \mathrm{O}\right)$ attenuation and less than $1 \%$ for the PMTR.

$\chi^{2}$ Cuts (10) and (11) : These systematic errors consisted in more restrictive $\chi^{2}$ cuts than the nominal fit $\left(\chi^{2}<25\right)$. They were useful to understand how the PMT selection affected the fit results. They do not account for physical uncertainties therefore there were no weighting factors applied $\left(f_{10}=f_{11}=1.0\right)$. The shifts in the attenuations and PMTR were of the order of 1-2\% such that these systematic errors could contribute up to $3 \%$ when added together. 


\section{A.2.2 PMT Efficiencies and Detector Response}

In Chapter 3, the systematic errors associated with the input efficiencies used with the Occupancy method and the asymmetry of the PMTR were evaluated with the NCD commissioning phase data. The same procedure was followed during the NCD phase.

PMT Efficiencies (12) : A scale of $10 \%$ was used to shift the efficiencies, corresponding to twice the statistical spread of the uncertainties in the efficiencies. The effect on the $\mathrm{D}_{2} \mathrm{O}$ attenuations was an increase greater than $30 \%$ for all wavelengths. The effect on the $\mathrm{H}_{2} \mathrm{O}$ attenuations cannot be interpreted directly because these attenuation lengths are correlated to the PMTR at large angle. Specifically in this case, the PMTR bins were systematically shifted down by $1 \%$ to $2.5 \%$. To accommodate the fact that the spread in the efficiencies was $5 \%$, not $10 \%$, a scale factor of $f_{12}=0.5$ was used. Although reduced after applying the weight, this systematic effect was one of the most important on the optical parameters.

PMT Response Asymmetry (13) : The PMTR was obtained independently for the top $(z>0 \mathrm{~cm})$ and bottom $(z<0 \mathrm{~cm})$. One problem in the NCD phase was the level of statistics in each bin of incident angle prevented from performing a precise extraction of independent PMTRs. Nevertheless, top-down differences as large as $4 \%$ were observed. Assuming there were statistical fluctuations in the $4 \%$ difference, the estimate of this effect was obtained, after applying a factor of $f_{13}=0.5$, to be $1 \%$ on the averaged PMT response. 


\section{A.2.3 New Systematic Errors for the NCD Phase}

OCAs in the NCD phase rely on the knowledge of the NCD positions to apply the shadow cut and correct PMT occupancies for reflections. Unsurprisingly the new systematic errors must incorporate the change in the optical parameters due to these new steps. In the following it is assumed that the systematic uncertainties of the LB positions has propagated into the uncertainties on the NCD positions, which allows one to treat the NCD effects independently from the systematic errors described in Subsection A.2.1.

\section{NCD Shadows}

The shadow cut used the fitted NCD positions and the mean radial uncertainty to flag shadowed PMTs through the tolerance $\Delta L \sim 5 \mathrm{~cm}$. The efficiency of the shadow cut must be tested by either shifting the NCD positions by amounts that are within their individual radial uncertainties, or equivalently by reducing the tolerance.

Tolerance Parameter (14) : For technical reasons, altering the tolerance is easier than shifting individual NCD positions. The tolerance value was set to $\Delta L^{\prime}=2.5 \mathrm{~cm}$, half of its nominal value. This allowed the effect of shadowed PMTs to be seen or, in the case where the cut efficiency was very high, the effect of including more statistics. The change affected the $\mathrm{D}_{2} \mathrm{O}$ attenuation primarily by about $2-3 \%$. Hence the efficiency of the cut was high, as wanted. Reducing the shadow tolerance from $5 \mathrm{~cm}$ to $2.5 \mathrm{~cm}$ yielded a conservative weighting factor of $f_{14}=0.5$. The same applies for the PMTR and generally to all wavelengths. 


\section{NCD Reflections}

The PMT occupancy correction was affected by the LB and NCD positions because of the geometry of the solid angles involved in the calculation of the probability $w_{i j}$. In addition, $w_{i j}$ contained a weight from the NCD surface reflectivity $R(\lambda)$. The two quantities were altered to obtain their effects on the parameters.

NCD Reflectivity (15): The reflectivity was set to $R(\lambda)=0$ to cancel the effect of the reflections. The occupancy correction then reduced to the pure MPE correction, but still with additional counts from the reflections in the data; this was approximately equivalent to fitting without applying the NCD reflection correction. A factor of $f_{15}=1$ was used to get the full systematic due to the modified correction. The shifts in the parameters were small, confirming that the reflectivity correction was small. The attenuations shifted slightly, but agreed with the nominal values considering the statistical uncertainties. The same rule applied for the PMTR.

Reflection Probability (16) : The reflection probability was multiplied by a factor of 10. This accounted for errors in the solid angles, time windows, and NCD reflectivity. This translated the facts that the NCD reflectivity scale was not known precisely, and that the acceptance calculation in the occupancy correction only modeled the diffuse nature of the reflections. The effect on the $\mathrm{D}_{2} \mathrm{O}$ attenuations was significant, and away from the reach of the statistical uncertainties of the nominal values. The increase in the $\mathrm{D}_{2} \mathrm{O}$ attenuations was of the order of $25 \%$. The $\mathrm{H}_{2} \mathrm{O}$ attenuations and PMTR were almost insensitive to that change, as expected; the absolute scale of the $\mathrm{NCD}$ reflectivity only affected the $\mathrm{D}_{2} \mathrm{O}$ attenuations. This effect was weighted with a 
factor of $f_{16}=0.2$, assuming the errors associated with this shift could not be greater than an equivalent increase in the NCD reflectivity of $100 \%$.

\section{A.2.4 Summary}

The systematic shifts of the previous subsections, independently of their numerical values, were automatically generated, calculated and weighted in the final results of Section 4.5. The contribution of each systematic was calculated from the weighted difference to the nominal fit, and the total systematic uncertainty was the quadrature sum of all systematic errors:

$$
\Delta_{\mathrm{syst}}^{p}=\sqrt{\sum_{i=1}^{N_{\mathrm{syst}}}\left(f_{i} \times\left|p_{i}-p_{0}\right|\right)^{2}},
$$

where $p_{0}$ and $p_{i}$ are the nominal and shifted parameters, and $f_{i}$ are the weights. Finally, the statistical fit uncertainty was added in quadrature to $\Delta_{\text {syst }}^{p}$ which gave the total error

$$
\Delta^{p}=\sqrt{\left(\Delta_{\text {syst }}^{p}\right)^{2}+\left(\Delta_{\text {stat }}^{p}\right)^{2}}
$$

The list of systematic uncertainties and their quantitative effects on the media attenuations and PMTR were summarized in Table 4.4. The following includes sample tables showing the fractional contribution of each of the 16 systematic errors to the total uncertainty $\Delta^{p}$.

\section{Tabulated Uncertainties}

Table A.2 shows the detailed decomposition of the errors on the $\mathrm{D}_{2} \mathrm{O}$ and $\mathrm{H}_{2} \mathrm{O}$ attenuations at 420 and $500 \mathrm{~nm}$ obtained from the February/05 scan. The errors extracted 
from the February/05 scan are among the largest throughout all selected scans. The systematic errors are identified by their indices given in Table A.1. The weighted differences $\delta_{i}=f_{i} \times\left|p_{i}-p_{0}\right|$ are given in percent for clarity. The total error on the $\mathrm{D}_{2} \mathrm{O}$ attenuation, $\alpha_{\mathrm{d}}$, gets a non-negligible contribution from the NCD phase systematic uncertainties. The main contribution comes from not knowing the NCD reflectivity precisely. As a result the $\mathrm{D}_{2} \mathrm{O}$ attenuations, sensitive to this input, are greatly affected.

The $\mathrm{H}_{2} \mathrm{O}$ attenuation sources of uncertainty are more spread, and less affected by the NCD effects. The total error is dominated by six systematic uncertainties, from which only one is a consequence of the NCD counters. The strongest $\chi^{2}$ cut $(i=11)$ contributes because it removes many PMTs in high radius runs which measure high incident angles in the PMTR function and $\alpha_{\mathrm{h}}$ simultaneously. The input efficiencies $(i=12)$ also affect the $\mathrm{H}_{2} \mathrm{O}$ attenuations for the same reason.

Table A.3 shows the detailed decomposition of the errors in the PMTR measurements at $10^{\circ}$ and $35^{\circ}$ at 365 and $386 \mathrm{~nm}$, obtained from the February/05 and May/05 scans. The main contribution in each case comes from the $\chi^{2}$ cut $(i=11)$, the input efficiencies $(i=12)$, and the up-down asymmetries $(i=13)$. Nevertheless, in general, the PMTR systematic uncertainty is of the same order as the statistical uncertainty. The PMTR is measured to better than $1 \%$ even with the additional contributions of the NCD-phase systematic uncertainties.

\section{A.3 Media Attenuation Lengths}

The numerical values from Figure 4.6 are given in this section showing the decomposition of the pure attenuation and Rayleigh scattering (RS) lengths. 


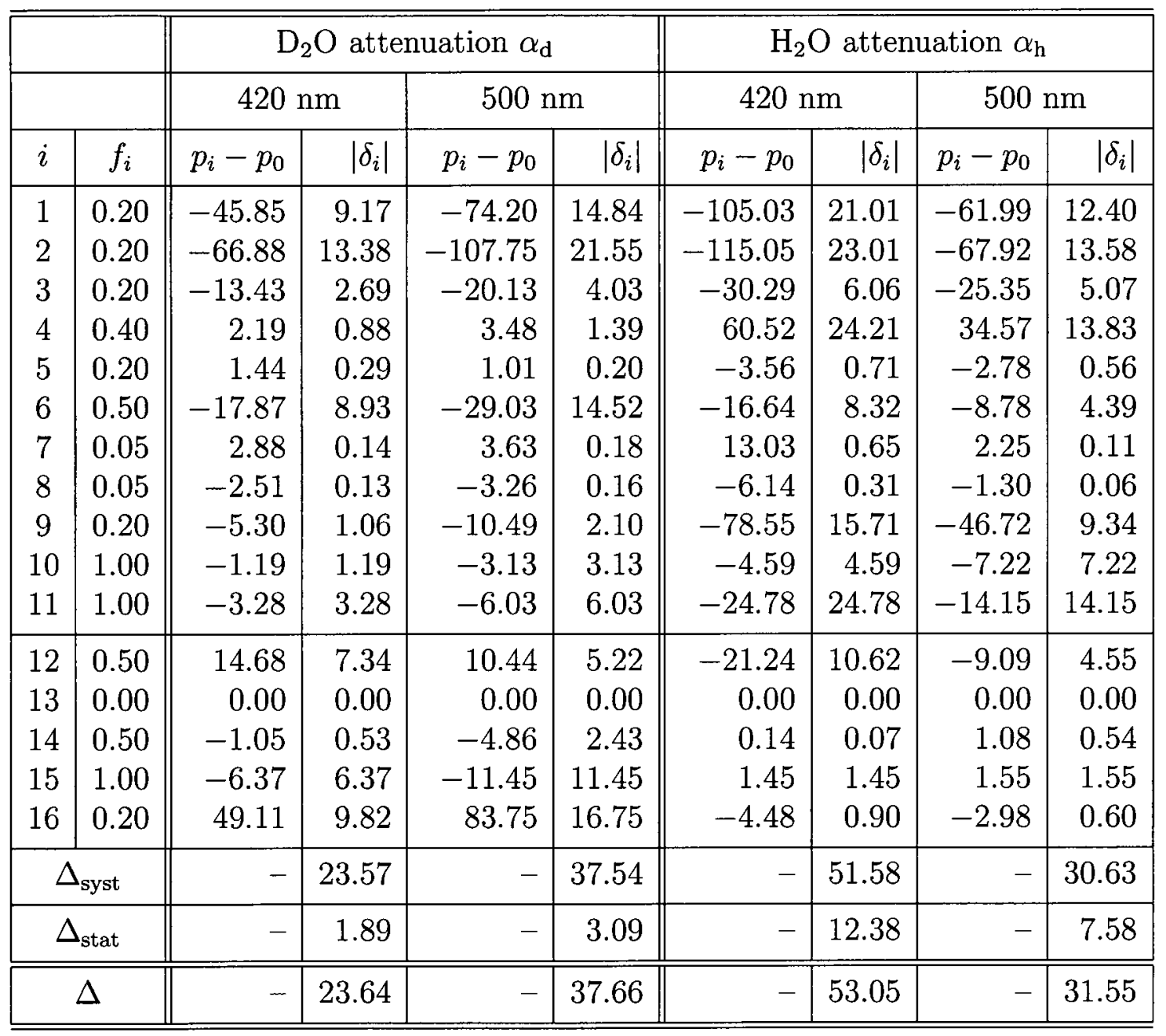

Table A.2: Decomposition of the systematic errors on the media attenuations obtained with the February/05 scan. The systematic indices $i$ correspond to those of Table A.1. The absolute differences $p_{i}-p_{0}$ are scaled by the factors $f_{i}$ to obtain the differences $\delta_{i}$. The differences and uncertainties are given in percent. 


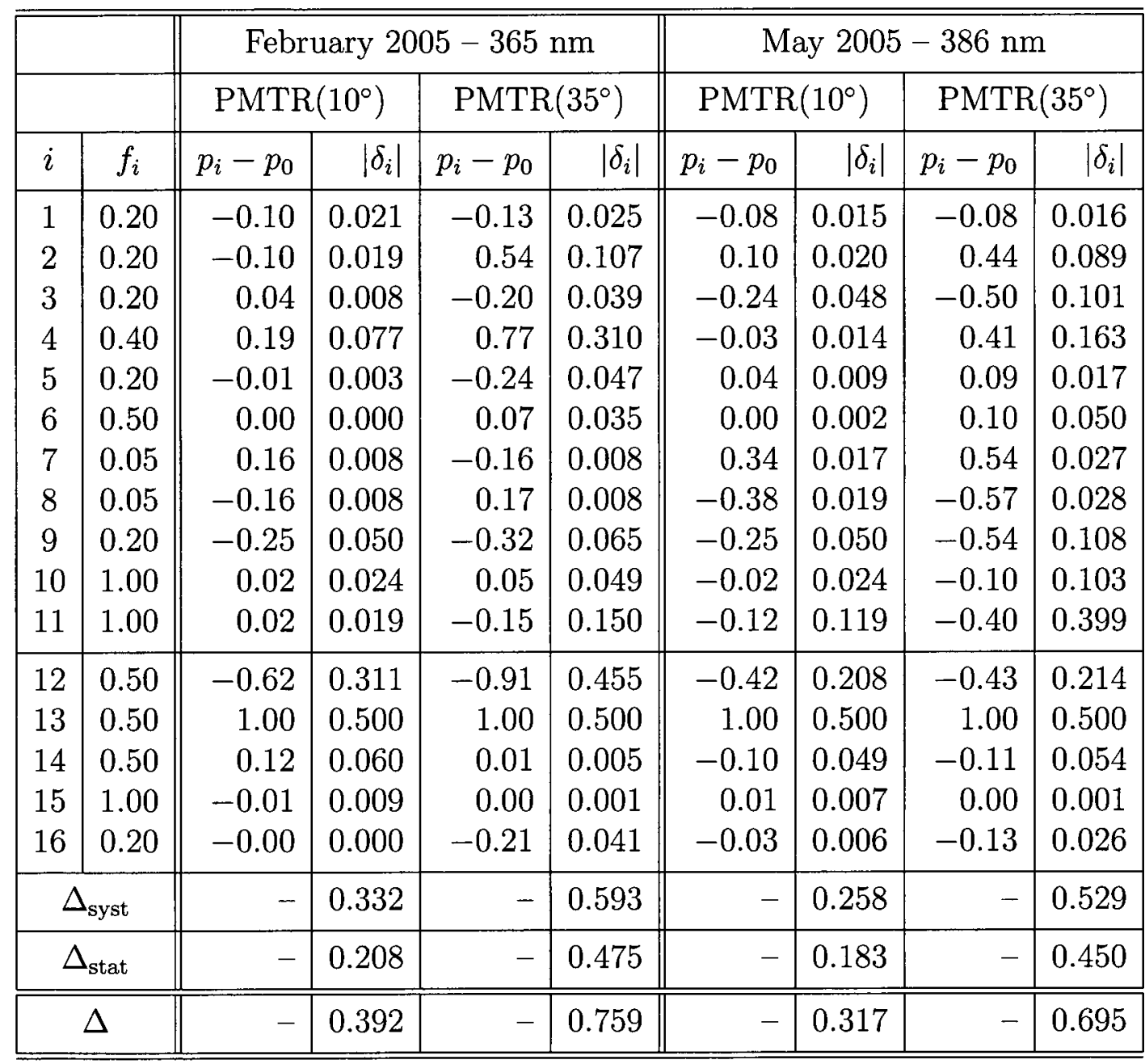

Table A.3: Decomposition of the systematic errors on the PMTR obtained with the February $/ 05$ and May/05 scans. The systematic indices $i$ correspond to those of Table A.1. The absolute differences $p_{i}-p_{0}$ are scaled by the factors $f_{i}$ to obtain the differences $\delta_{i}$. The differences and uncertainties are given in percent. 


\begin{tabular}{|c||r|r|r|r|r|r|r|}
\hline \hline$\lambda(\mathrm{nm})$ & October 2003 & October 2004 & February 2005 & May 2005 & February 2006 & August 2006 & NCD Average \\
\hline \hline 337 & $16.22 \pm 1.24$ & $11.71 \pm 1.33$ & $13.19 \pm 1.61$ & $12.99 \pm 1.37$ & $12.35 \pm 1.23$ & $12.81 \pm 1.43$ & $12.55 \pm 0.62$ \\
365 & $12.05 \pm 1.21$ & $7.96 \pm 1.30$ & $10.52 \pm 1.52$ & $10.01 \pm 1.34$ & $9.14 \pm 1.24$ & $10.72 \pm 1.40$ & $9.58 \pm 0.60$ \\
386 & $10.46 \pm 1.19$ & $7.40 \pm 1.30$ & $8.92 \pm 1.50$ & $8.60 \pm 1.32$ & $8.33 \pm 1.27$ & $9.10 \pm 1.38$ & $8.43 \pm 0.60$ \\
420 & $7.86 \pm 1.19$ & $4.99 \pm 1.29$ & $6.43 \pm 1.52$ & $6.85 \pm 1.33$ & $3.84 \pm 1.27$ & $6.93 \pm 1.36$ & $5.72 \pm 0.60$ \\
500 & $5.17 \pm 1.19$ & $2.49 \pm 1.32$ & $3.89 \pm 1.47$ & $3.29 \pm 1.30$ & $3.34 \pm 1.26$ & $4.01 \pm 1.33$ & $3.38 \pm 0.60$ \\
620 & $5.23 \pm 1.16$ & $2.92 \pm 1.47$ & $5.51 \pm 1.94$ & $5.66 \pm 1.66$ & $3.60 \pm 1.45$ & $5.28 \pm 1.59$ & $4.41 \pm 0.71$ \\
\hline \hline
\end{tabular}

Table A.4: $\mathrm{D}_{2} \mathrm{O}$ inverse total extinction lengths in the NCD phase, in units of $10^{-5} \mathrm{~cm}^{-1}$.

\begin{tabular}{|c||r|r|r|r|r|r|r|}
\hline \hline$\lambda(\mathrm{nm})$ & October 2003 & October 2004 & February 2005 & May 2005 & February 2006 & August 2006 & NCD Average \\
\hline \hline 337 & $5.26 \pm 1.71$ & $-0.24 \pm 1.36$ & $1.36 \pm 1.43$ & $1.41 \pm 1.47$ & $1.51 \pm 1.68$ & $2.11 \pm 1.23$ & $1.24 \pm 0.63$ \\
365 & $3.19 \pm 1.17$ & $-1.23 \pm 1.25$ & $1.55 \pm 1.39$ & $1.47 \pm 1.24$ & $0.89 \pm 1.51$ & $2.57 \pm 1.16$ & $1.09 \pm 0.58$ \\
386 & $3.89 \pm 1.35$ & $0.27 \pm 1.33$ & $2.31 \pm 1.46$ & $2.23 \pm 1.23$ & $2.07 \pm 1.53$ & $2.04 \pm 1.09$ & $1.79 \pm 0.58$ \\
420 & $3.96 \pm 1.36$ & $0.65 \pm 1.28$ & $2.62 \pm 1.39$ & $2.58 \pm 1.21$ & $1.13 \pm 1.54$ & $2.54 \pm 1.16$ & $1.97 \pm 0.58$ \\
500 & $5.94 \pm 1.32$ & $2.66 \pm 1.27$ & $4.35 \pm 1.37$ & $3.95 \pm 1.22$ & $4.78 \pm 1.47$ & $4.70 \pm 1.05$ & $4.10 \pm 0.56$ \\
620 & $33.21 \pm 1.70$ & $29.54 \pm 1.72$ & $29.85 \pm 2.03$ & $29.94 \pm 1.77$ & $30.60 \pm 1.91$ & $31.96 \pm 1.31$ & $30.62 \pm 0.75$ \\
\hline
\end{tabular}

Table A.5: $\mathrm{H}_{2} \mathrm{O}$ inverse total extinction lengths in the NCD phase, in units of $10^{-4} \mathrm{~cm}^{-1}$.

\section{A.3.1 Total Extinctions}

Tables A.4 and A.5 give the total extinction lengths in the $\mathrm{D}_{2} \mathrm{O}$ and $\mathrm{H}_{2} \mathrm{O}$ measured in the NCD phase at the six wavelengths available with the SNO calibration system.

\section{A.3.2 Pure Media Attenuation Lengths}

Table A.7 gives the values of the pure media attenuation lengths after the RS lengths from Table A.6 were subtracted. The fraction of RS that contribute to the prompt peak are $0.82,1.00$, and 1.03 in the $\mathrm{D}_{2} \mathrm{O}$, acrylic, and $\mathrm{H}_{2} \mathrm{O}$, respectively. These fractions correspond to the values measured in the $\mathrm{D}_{2} \mathrm{O}$ phase. 


\begin{tabular}{|c||c|c|c|c|c|c|}
\hline \hline \multicolumn{1}{|c||}{} & \multicolumn{3}{c|}{ Measured } & \multicolumn{3}{c|}{ Calculated } \\
\hline$\lambda(\mathrm{nm})$ & $\mathrm{D}_{2} \mathrm{O}$ & Acrylic & $\mathrm{H}_{2} \mathrm{O}$ & $\mathrm{D}_{2} \mathrm{O}$ & Acrylic & $\mathrm{H}_{2} \mathrm{O}$ \\
\hline \hline 337 & 13.6225 & 56.40 & 17.5594 & 8.19450 & 29.6955 & 8.04839 \\
365 & 9.55891 & 23.00 & 12.3214 & 5.93698 & 20.8544 & 5.83747 \\
386 & 7.48047 & 12.20 & 9.64233 & 4.73799 & 16.3286 & 4.66271 \\
420 & 5.13529 & 7.70 & 6.61939 & 3.37214 & 11.3332 & 3.32363 \\
500 & 2.46663 & 7.09 & 3.17948 & 1.67239 & 5.39509 & 1.65476 \\
620 & 1.00171 & 7.09 & 1.29120 & 0.704985 & 2.19365 & 0.701905 \\
\hline \hline
\end{tabular}

Table A.6: Reference values of Rayleigh scattering inverse attenuation lengths in units of $10^{-5} \mathrm{~cm}^{-1}$. Measured values in $\mathrm{D}_{2} \mathrm{O}$ and $\mathrm{H}_{2} \mathrm{O}$ are taken from [116]. Measured values in acrylic are from $[30,56]$, where the values are reported from ex situ measurements using the SNO AV panels before the construction of the SNO detector. Theoretical values are calculated with the QOptics code.

\section{A.3.3 Contributions from NCD Reflections}

In the nominal fit, a correction is applied to the data, which makes the nominal total extinctions free of NCDR effects. In that latter case, only a fraction of RS needs to be subtracted to obtain the pure media attenuation lengths. The NCDR correction can be turned off to obtain attenuation with the effects of the reflections. Knowing the difference between the nominal attenuations and the attenuations obtained without correction allows one to estimate the absorption length due to the NCDRs, assuming the RS is present in the same proportion. The terminology absorption length is used although this quantity must be negative for NCDRs because they add counts to the occupancy. The NCDR absorption length, $\alpha^{\mathrm{NCDR}}$, is the difference:

$$
\alpha_{m}^{\mathrm{NCDR}}=\alpha_{m}^{R(\lambda)=0}-\alpha_{m},
$$




\begin{tabular}{|l|l|c|c|c|c|c|c|}
\hline \hline Media & Scan & $337 \mathrm{~nm}$ & $365 \mathrm{~nm}$ & $386 \mathrm{~nm}$ & $420 \mathrm{~nm}$ & $500 \mathrm{~nm}$ & $620 \mathrm{~nm}$ \\
\hline \hline \multirow{5}{*}{$\mathrm{D}_{2} \mathrm{O}$} & October 2003 & 5.533 & 4.596 & 4.399 & 3.610 & 3.081 & 4.197 \\
\cline { 2 - 8 } & October 2004 & 0.5377 & 0.1234 & 1.262 & 0.7761 & 0.4657 & 2.096 \\
& February 2005 & 2.015 & 2.684 & 2.784 & 2.215 & 1.872 & 4.688 \\
& May 2005 & 1.823 & 2.175 & 2.462 & 2.641 & 1.270 & 4.836 \\
& February 2006 & 1.184 & 1.303 & 2.200 & -0.3749 & 1.316 & 2.774 \\
& August 2006 & 1.643 & 2.881 & 2.969 & 2.717 & 1.991 & 4.463 \\
\cline { 2 - 8 } & NCD Average & 1.383 & 1.739 & 2.292 & 1.504 & 1.361 & 3.593 \\
\hline \hline \multirow{5}{*}{$\mathrm{H}_{2} \mathrm{O}$} & October 2003 & 19.58 & 16.95 & 28.65 & 31.94 & 60.21 & 321.8 \\
\cline { 2 - 8 } & October 2004 & -10.69 & -18.29 & -2.053 & 3.027 & 24.88 & 294.7 \\
& February 2005 & 5.343 & 9.454 & 18.32 & 22.82 & 41.84 & 297.8 \\
& May 2005 & 5.828 & 8.728 & 17.52 & 22.34 & 37.80 & 298.6 \\
& February 2006 & 6.856 & 2.851 & 15.93 & 7.851 & 4.608 & 305.3 \\
& August 2006 & 12.80 & 19.72 & 15.55 & 21.99 & 45.28 & 318.9 \\
\cline { 2 - 8 } & NCD Average & 4.142 & 4.915 & 13.12 & 16.30 & 39.28 & 305.5 \\
\hline \hline
\end{tabular}

Table A.7: Media pure inverse attenuations lengths in the NCD phase, in units of $10^{-5} \mathrm{~cm}^{-1}$. The amount of Rayleigh scattering assumed is 0.82 for $\mathrm{D}_{2} \mathrm{O}$ and 1.03 for $\mathrm{H}_{2} \mathrm{O}$.

\begin{tabular}{|c|c|c|}
\hline \hline$\lambda(\mathrm{nm})$ & $\alpha_{\mathrm{d}}^{\mathrm{NCDR}}$ & $\alpha_{\mathrm{h}}^{\mathrm{NCDR}}$ \\
\hline 337 & $(-1.96 \pm 1.01) \times 10^{-6}$ & $(+3.39 \pm 27.6) \times 10^{-6}$ \\
365 & $(-2.08 \pm 0.86) \times 10^{-6}$ & $(+4.29 \pm 23.3) \times 10^{-6}$ \\
386 & $(-2.14 \pm 0.80) \times 10^{-6}$ & $(+7.36 \pm 21.9) \times 10^{-6}$ \\
421 & $(-2.40 \pm 0.77) \times 10^{-6}$ & $(+7.29 \pm 20.3) \times 10^{-6}$ \\
500 & $(-2.77 \pm 0.76) \times 10^{-6}$ & $(+5.01 \pm 20.8) \times 10^{-6}$ \\
620 & $(-3.28 \pm 0.78) \times 10^{-6}$ & $(+7.40 \pm 21.7) \times 10^{-6}$ \\
\hline \hline
\end{tabular}

Table A.8: Average absorption coefficients from NCD reflections in units of $\mathrm{cm}^{-1}$. 
where $m$ is the medium index $\left(\mathrm{D}_{2} \mathrm{O}\right.$, acrylic, and $\left.\mathrm{H}_{2} \mathrm{O}\right)$ and the notation $R(\lambda)=0$ means the NCD reflectivity is zero which turns off the correction. Table A.8 gives the $\mathrm{D}_{2} \mathrm{O}$ and $\mathrm{H}_{2} \mathrm{O}$ NCD-phase averages and weighted errors for all wavelengths. The error in $\alpha_{m}^{\mathrm{NCDR}}$ is taken as the sum in quadrature of the statistical errors for both $\alpha_{m}^{R(\lambda)=0}$ and $\alpha_{m}$. The negative inverse attenuation lengths obtained in the $\mathrm{D}_{2} \mathrm{O}$ are smaller than the pure attenuation lengths by an order of magnitude. Although the effect is small, the total statistical errors indicate that the effect can be measured above statistical fluctuations. On the other hand, the $\mathrm{H}_{2} \mathrm{O}$ values are all consistent with zero since the errors are large. Plots of $\alpha_{d}^{\mathrm{NCDR}}$ against wavelength [97] showed that the effect followed a polynomial function, in agreement with the function $R(\lambda)$ in Figure 4.5a.

\section{A.4 PMT Angular Response}

The grey disk (GD) model in SNOMAN is based on ex situ PMT response measurements [75] and the result of the optical calibrations. This section explains how the PMT response bank, PMTR, read by SNOMAN, is constructed from various measurements.

\section{Quantum Efficiency}

The PMT quantum efficiency (QE) was measured in [75] and is plotted in Figure A.1a relative to its maximum value of $21.5 \%$. The $\mathrm{QE}$ as a function of wavelength is used to rescale the wavelength dependence of the relative PMTR functions, from which the QE has been already taken into account in the fit. 


\section{Angle of Incidence Extrapolation}

Optical calibrations cannot measure the PMTR beyond $45^{\circ}$ from LB positions inside the AV. Although there were positions taken outside the AV, in the $\mathrm{H}_{2} \mathrm{O}$, the data were never fully understood due to the complex reflection patterns on the exterior of the AV, PMT reflectors, and PSUP. To enable the response to all angle of incidence in the simulation, the ex situ measurements are used to extrapolate the function up to $90^{\circ}$. The scale of the optical calibration measurement has predominance. The $e x$ situ curve is scaled such that the last bin matches the optics measurement, and from that point, the extrapolation curve is inserted and used as the PMTR. Figure A.1b shows the result of the procedure for an arbitrary measurement. The shaded part is the extrapolation from the ex situ curve.

\section{PMTR Surface}

Both the QE profile and extrapolated PMTR curves are used for all six wavelengths of the calibration to build a 2D PMTR surface. Figure A.2 shows the a surface read by SNOMAN when calculating the GD model probabilities. The scale of the figure is relative since the absolute PMT collection efficiency is set using ${ }^{16} \mathrm{~N}$ calibrations rather than the PMT response measurements. However the shape of the PMTR function is kept intact, and SNOMAN interpolates between the bins to access a particular probability as a function of both incident angle and wavelength. 


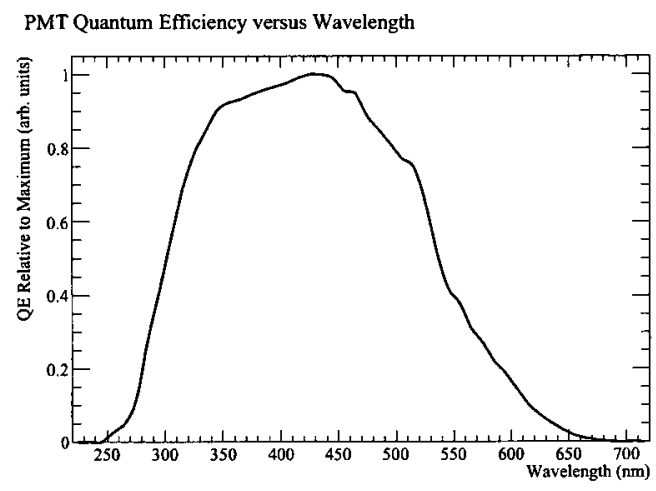

(a) Relative quantum efficiency.

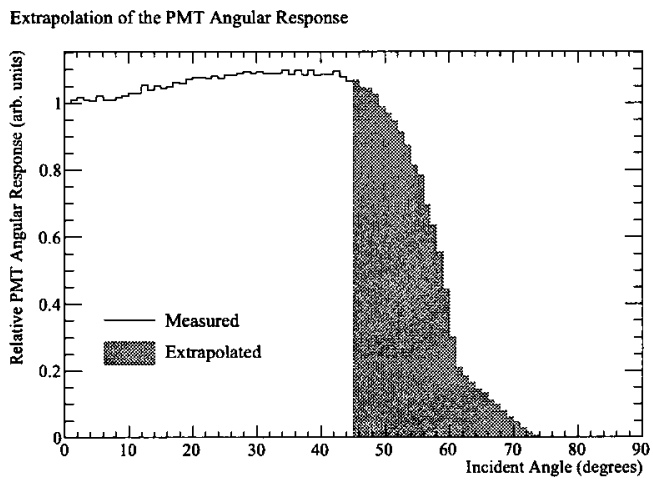

(b) PMTR extrapolation.

Figure A.1: PMT quantum efficiency and angular response extrapolation.

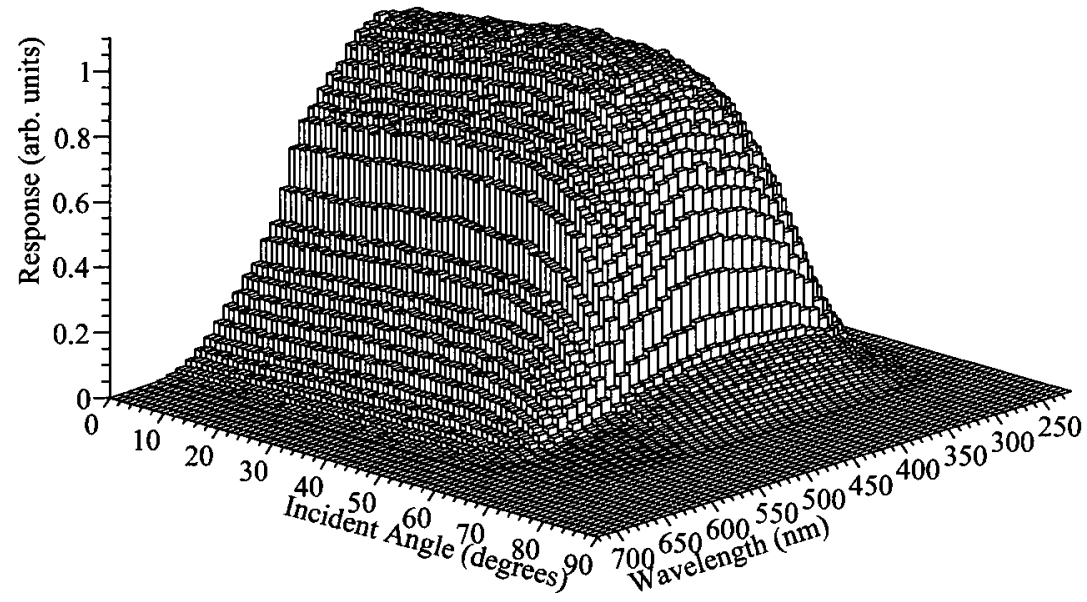

Figure A.2: PMT response surface as a function of wavelength and incident angle in the PMTR bank read by SNOMAN. 


\section{APPENDIX B}

\section{Parameters in Signal Extraction}

The variables names of the fitted fraction of signal events, with energy ranges and zenith orientations, background events, and systematic parameters are given in Table B.1 for reference to Chapter 5.

The $\mathrm{PMT}_{\gamma}^{\beta}$ background PDF was parameterized with an analytic shape given by:

$$
f\left(T_{\text {eff }}, \beta_{14}, \rho\right)=\exp \left\{\epsilon T_{\text {eff }}\right\} \times N\left(\beta_{14} \mid \omega_{0}+\omega_{1} \rho, \beta_{\text {sm }}\right) \times(B+\exp \{\nu \rho\})
$$

where $N(\mu, \sigma)$ is a normal distribution with the given mean $\mu=\bar{\beta}_{14}=\omega_{0}+\omega_{1} \rho$ and width $\sigma=\beta_{s m}$. The PDF was assumed to be constant (flat) in $\cos \theta_{\odot}$. 


\begin{tabular}{|c|c|c|}
\hline Signals & $\begin{array}{l}\text { CC-D }\left[T_{\mathrm{eff}}^{\min }, T_{\mathrm{eff}}^{\max }\right] \\
\text { CC-N }\left[T_{\mathrm{eff}}^{\min }, T_{\mathrm{eff}}^{\max }\right] \\
\text { ES-D }\left[T_{\mathrm{ef}}^{\min }, T_{\mathrm{eff}}^{\max }\right] \\
\text { ES-N }\left[T_{\mathrm{eff}}^{\min }, T_{\mathrm{eff}}^{\max }\right] \\
\text { NC-A }\end{array}$ & $\begin{array}{l}\text { Unconstrained CC day bin with } T_{\text {eff }} \text { range } \\
\text { Unconstrained CC night bin with } T_{\text {eff }} \text { range } \\
\text { Unconstrained ES day bin with } T_{\text {eff }} \text { range } \\
\text { Unconstrained ES night bin with } T_{\text {eff }} \text { range } \\
\text { Constrained NC average in the full } T_{\text {eff }} \text { range }\end{array}$ \\
\hline BGs & $\begin{array}{l}\text { d2o_av_neutrons } \\
\text { d2o_bi_d2o } \\
\text { d2o_tl_d2o } \\
\text { d2o_bi_av_bulk } \\
\text { d2o_tl_av_bulk } \\
\text { d2o_bi_h2o } \\
\text { d2o_tl_h2o } \\
\text { salt_av_neutrons } \\
\text { salt_bi_d2o } \\
\text { salt_tl_d2o } \\
\text { salt_24na } \\
\text { salt_bi_av_bulk } \\
\text { salt_tl_av_bulk } \\
\text { salt_bi_h2o } \\
\text { salt_tl_h2o } \\
\text { d2o_pmt } \\
\text { salt_pmt }\end{array}$ & $\begin{array}{l}\text { Neutrons from the AV surface in the } \mathrm{D}_{2} \mathrm{O} \text { phase } \\
\text { Internal } \mathrm{Bi} \text { in the } \mathrm{D}_{2} \mathrm{O} \text { phase } \\
\text { Internal } \mathrm{Tl} \text { in the } \mathrm{D}_{2} \mathrm{O} \text { phase } \\
\mathrm{Bi} \text { from the } \mathrm{AV} \text { in the } \mathrm{D}_{2} \mathrm{O} \text { phase } \\
\mathrm{Tl} \text { from the } \mathrm{AV} \text { in the } \mathrm{D}_{2} \mathrm{O} \text { phase } \\
\text { External } \mathrm{Bi} \text { in the } \mathrm{D}_{2} \mathrm{O} \text { phase } \\
\text { External } \mathrm{Tl} \text { in the } \mathrm{D}_{2} \mathrm{O} \text { phase } \\
\text { Neutrons from the } \mathrm{AV} \text { surface in the salt phase } \\
\text { Internal } \mathrm{Bi} \text { in the salt phase } \\
\text { Internal } \mathrm{Tl} \text { in the salt phase } \\
\text { Neutrons from }{ }^{24} \mathrm{Na} \text { in the salt phase } \\
\mathrm{Bi} \text { from the AV in the salt phase } \\
\text { Tl from the AV in the salt phase } \\
\text { External } \mathrm{Bi} \text { in the salt phase } \\
\text { External Tl in the salt phase } \\
\mathrm{PMT}_{\gamma}^{\beta} \text { in the } \mathrm{D}_{2} \mathrm{O} \text { phase } \\
\mathrm{PMT}_{\gamma}^{\beta} \text { in the salt phase }\end{array}$ \\
\hline Systs & $\begin{array}{l}\text { escale_u } \\
\text { d2o_escale_u } \\
\text { salt_escale_u } \\
\text { d2o_eres } \\
\text { salt_eres_e } \\
\text { salt_eres_n } \\
\text { b14scale0eD20n_u } \\
\text { b14scale0Saltn } \\
\text { b14scale1 } \\
\text { d2o_pmt_epsilon_m } \\
\text { salt_pmt_epsilon_m } \\
\text { d2o_pmt_omega0_m } \\
\text { salt_pmt_omega0_m } \\
\text { d2o_pmt_nu_m } \\
\text { salt_pmt_nu_m } \\
\text { d2o_pmt_b_m } \\
\text { salt_pmt_b_m }\end{array}$ & $\begin{array}{l}\text { Energy scale (common) } \\
\text { Energy scale in the } \mathrm{D}_{2} \mathrm{O} \text { phase } \\
\text { Energy scale in the salt phase } \\
\text { Energy resolution in the } \mathrm{D}_{2} \mathrm{O} \text { phase } \\
\text { Energy resolution to electrons in the salt phase } \\
\text { Energy resolution to neutrons in the salt phase } \\
\beta_{14} \text { scale for electrons and } \mathrm{D}_{2} \mathrm{O} \text {-phase neutrons } \\
\beta_{14} \text { scale for salt-phase neutrons } \\
\beta_{14} \text { dependence on } T_{\text {eff }} \\
\mathrm{PMT}_{\gamma}^{\beta} \in \text { parameter in the } \mathrm{D}_{2} \mathrm{O} \text { phase } \\
\mathrm{PMT}_{\gamma}^{\beta} \in \text { parameter in the salt phase } \\
\mathrm{PMT}_{\gamma}^{\beta} \omega_{0} \text { parameter in the } \mathrm{D}_{2} \mathrm{O} \text { phase } \\
\mathrm{PMT}_{\gamma}^{\beta} \omega_{0} \text { parameter in the salt phase } \\
\mathrm{PMT}_{\gamma}^{\beta} \nu \text { parameter in the } \mathrm{D}_{2} \mathrm{O} \text { phase } \\
\mathrm{PMT}_{\gamma}^{\beta} \nu \text { parameter in the salt phase } \\
\mathrm{PMT}_{\gamma}^{\beta} B \text { parameter in the } \mathrm{D}_{2} \mathrm{O} \text { phase } \\
\mathrm{PMT}_{\gamma}^{\beta} B \text { parameter in the salt phase }\end{array}$ \\
\hline
\end{tabular}

Table B.1: Name and description of the parameters in the signal extraction procedure of Chapter 5. The $\mathrm{PMT}_{\gamma}^{\beta}$ parameters can be found in Equation (B.1). 


\section{APPENDIX C}

\section{More on the Survival Probability and Oscillation Parameters}

This appendix gives technical details complementary to the analyses presented in Chapters 6 and 7. Section C.1 is specific to Chapter 6 and Section C. 2 to Chapter 7.

\section{C.1 More on Survival Probabilities}

This section refers to Chapter 6. Subsection C.1.1 discusses the SNO neutron response analytic function. Subsection C.1.2 explains the method to set the number of bins

of the binned survival probability function in Equation (6.10). Subsection C.1.3 describes and lists the rates from other solar neutrino experiments that are included in the global fit of the survival probability, but also in the global fit of the oscillation parameters presented in Chapter 7 .

\section{C.1.1 SNO Response to Neutrons}

The neutron response function was not necessary in the rate calculation of Equation $(6.4 \mathrm{~b})$ since the integration of the normalized $1 \mathrm{D}$ function effectively removed the effect of the NC signal shape as a function of $T \equiv T_{\text {eff }}$. However the response is 
given below for completeness.

The energy of the neutron and $\gamma$ 's emitted by the capture reaction are uncorrelated such that the neutron response only depends on $T$ :

$$
R^{n}(T)=\frac{1}{\sqrt{2 \pi}}\left\{\frac{q}{\sigma_{1}} \exp \left[-\frac{\left(\mu_{1}-T\right)^{2}}{2 \sigma_{1}^{2}}\right]+\frac{1-q}{\sigma_{2}} \exp \left[-\frac{\left(\mu_{2}-T\right)^{2}}{2 \sigma_{2}^{2}}\right]\right\}
$$

where $T$ can be affected by energy scale and offset parameters, as in the electron response case. The general neutron double-normal response was implemented for the salt phase, because of the $\gamma$-multiplicity, but was assumed to follow a single distribution for the $\mathrm{D}_{2} \mathrm{O}$ and NCD phases by setting $q=1$. Figure C.1 shows the neutron response obtained from the three phases of SNO, where $q=0.5433$ [156] for the salt phase. The three distributions are proportional to the amounts of MC neutron events that were available in each phase. The fits are not particularly good and in general the behavior in the tails are not well described by the response of Equation (C.1). Therefore the MC distributions, or PDFs made from them, are a better representation of the neutron response than the analytic function.

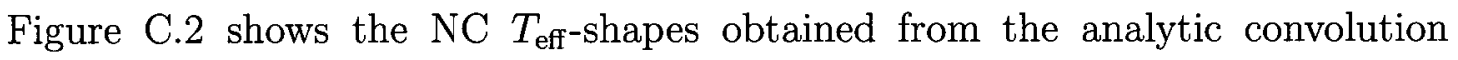
method compared to the MC simulation. They do not agree extremely well but these differences did not affect the integrated NC rate calculations.

\section{C.1.2 Survival Probability Binned Function}

The number of bins $N$ in Equation (6.10) and their widths were set by measuring the change in the shape of the SNO CC spectrum predicted by the analytic rate calculation. A typical LMA-region survival probability function (c.f. Chapter 7) was used to 


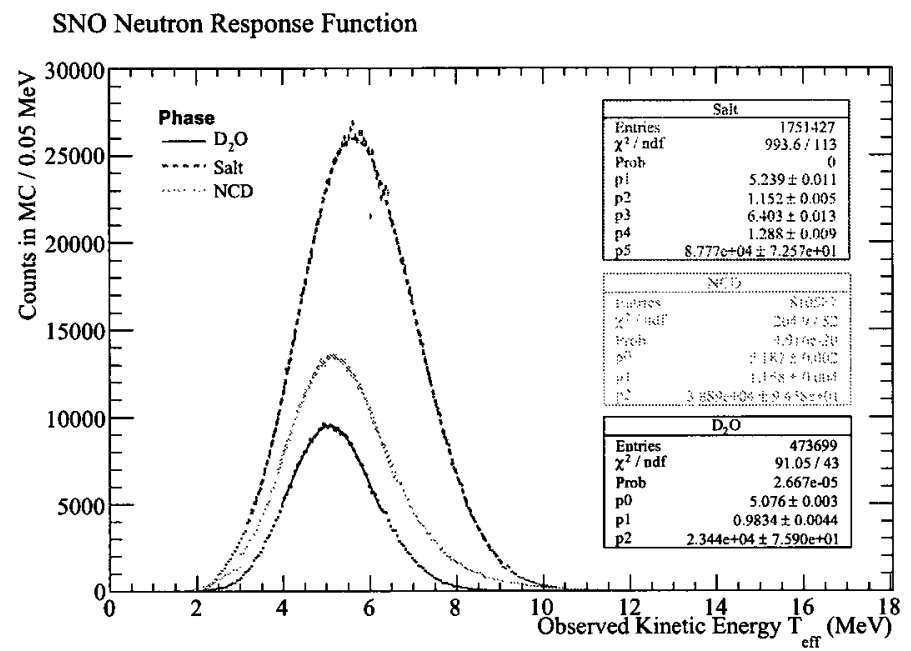

Figure C.1: SNO neutron response function. In the $\mathrm{D}_{2} \mathrm{O}$ and salt phases, the energy estimator is FTK; in the NCD phase the energy estimator is RSP.

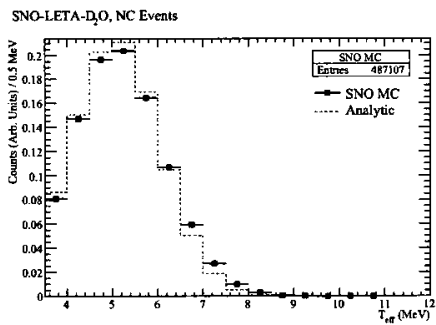

(a) $\mathrm{D}_{2} \mathrm{O} \mathrm{NC}$.

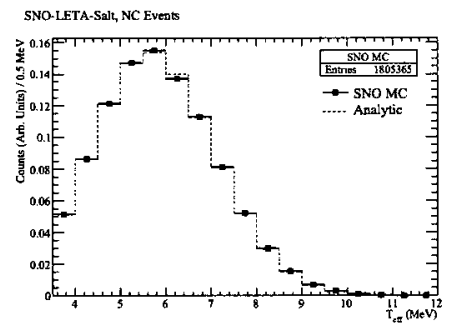

(b) Salt NC.

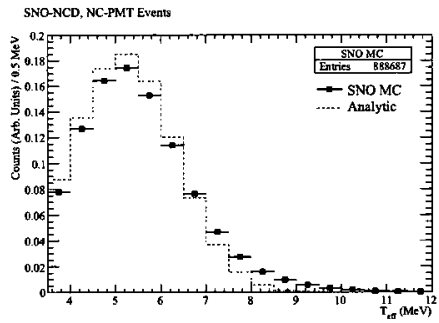

(c) NCD NC.

Figure C.2: Comparison of the SNO NC signal shapes obtained from the analytic model and MC simulation. The horizontal axis is $T_{\text {eff }}$ from 3.5 to $12 \mathrm{MeV}$.

calculate the nominal $\mathrm{CC}$ spectrum in $T_{\text {eff }}$, as shown in Figure C.3. The uncertainty band represents the uncertainties in the $\nu\left({ }^{8} \mathrm{~B}\right)$ spectrum shape, energy scale, and resolution, calculated by smearing the detector electron response parameters. The smooth survival probability curve was gradually binned from a large number of bins $(N \rightarrow \infty)$ to a single bin. For each binned function a new CC shape was obtained 


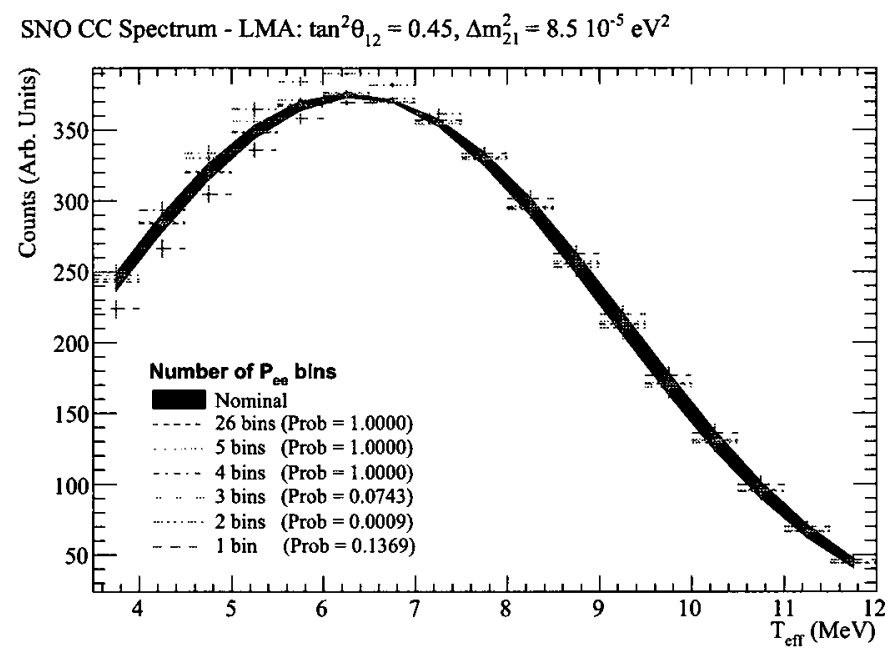

Figure C.3: Effect of binning the survival probability on the expected SNO CC spectrum. The nominal uncertainties are shown as an uncertainty band.

and compared to the nominal shape quantitatively using the $\chi^{2}$ function:

$$
\chi_{\text {shape }}^{2}=\sum_{i}^{N_{\text {bins }}^{\mathrm{CC}}} \frac{\left(\mathrm{CC}_{i}(N)-\mathrm{CC}_{i}(\infty)\right)^{2}}{\sigma_{i}^{2}(N)+\sigma_{i}^{2}(\infty)}
$$

where $N_{\text {bins }}^{\mathrm{CC}}=16$ is the number of $T_{\text {eff }}$ bins, and $N$ is the number of bins in $P_{b}\left(E_{\nu}\right)$. Requiring that the probability calculated from $\chi_{\text {shape }}^{2}$ obtained for each binned function shown in Figure C.3 must be higher than $\left(1-10^{-5}\right)$, the minimum number of bins that does not affect the CC spectrum considerably was found to be $N_{\min }=4$. However, wider bin sizes gave better results at low and high $E_{\nu}$ due to the low effective response below $6 \mathrm{MeV}$ and above $13 \mathrm{MeV}$. Therefore, $N=5$ weights were used in Equation (6.10), with three $2-\mathrm{MeV}$-wide bins in the most sensitive range in neutrino energy. 


\section{C.1.3 Solar Neutrino Experiments}

Subsection 6.3.2 referred to solar neutrino data taken from external sources. The data from these experiments are tabulated in Table C.1, as found in the publications released by the respective collaborations. Each experiment's normalization was adapted to match the BS05(OP) SSM prediction for the purpose of this thesis. In general these normalizations can be rescaled to match any model, assuming the original shape of the neutrino spectra did not change. The fractions $F_{n}^{\mathbf{e x}}$ with respect to the SSM prediction were obtained after dividing the rates by the normalizations.

In Table C.1, the Homestake and Gallium rates are given in SNUs; the Borexino rate is given in counts per day per 100-ton. The SK rates were obtained by adding the $\nu\left({ }^{8} \mathrm{~B}\right)$ and $\nu$ (hep) components together and are given in units of events per kiloton per year.

The SK-I rate groups $1-7,8-14,15-21,22-28,29-35$, and $36-42$ correspond to the observed total energy ranges $[5.5,6.5],[6.5,8.0],[8.0,9.5],[9.5,11.5],[11.5,13.5]$, and $[13.5,16.0] \mathrm{MeV}$, respectively. Each rate within these energies correspond to different zenith angle orientations, in order, from day $\left(\cos \theta_{z} \in[-1,0]\right)$ to mantle/core layers, $\cos \theta_{z} \in[0,0.16],[0.16,0.33],[0.33,0.50],[0.50,0.67],[0.67,0.84],[0.84,1]$, respectively. The rates 0 and 43 correspond to energies between $[5.0,5.5]$ and $[16,20] \mathrm{MeV}$, and are averaged over all orientations.

The SK-II rates are ordered according to the day/night orientation, and the energy bins are typically $0.5-\mathrm{MeV}$ wide with an analysis threshold of $7.0 \mathrm{MeV}$. For instance, rate 0 is averaged with $E \in[7.0,7.5] \mathrm{MeV}$, rates 1 and 2 are the day and night rates with $E \in[7.5,8.0] \mathrm{MeV}$, and so forth. 


\begin{tabular}{|c|c|c|c|c|c|c|}
\hline Experiment & $n$ & Meas. & Norm. & $n$ & Meas. & Norm. \\
\hline Homestake [13] & 0 & $2.56 \pm 0.23$ & 8.12 & & & \\
\hline Gallium [16] & 0 & $66.1 \pm 3.1$ & 126.08 & & & \\
\hline Borexino [39] & 0 & $49 \pm 5$ & 74 & & & \\
\hline \multirow[t]{22}{*}{ SK-I [19] } & 0 & $74.7_{-6.5}^{+6.6}$ & 180.06 & 22 & $57.1+1.9$ & 141.56 \\
\hline & 1 & $127 \pm 6$ & 315.08 & 23 & $\begin{array}{l}56.5+5.2 \\
-4.8\end{array}$ & 141.56 \\
\hline & 2 & $124 \pm 15$ & 315.08 & 24 & $\begin{array}{l}63.3 \pm 5.0 \\
-4.6\end{array}$ & 141.56 \\
\hline & 3 & $106+14$ & 315.08 & 25 & $56.8+4.9$ & 141.56 \\
\hline & 4 & $132+{ }_{12}^{+13}$ & 315.08 & 26 & $59.6_{-3.9}^{+4.2}$ & 141.56 \\
\hline & 5 & $146 \pm 12$ & 315.08 & 27 & $60.1+4.6$ & 141.56 \\
\hline & 6 & $140 \pm 13$ & 315.08 & 28 & $60.9+5.2$ & 141.56 \\
\hline & 7 & $119+15$ & 315.08 & 29 & $18.7+0.9$ & 44.01 \\
\hline & 8 & $149+4$ & 352.62 & 30 & $20.0 \pm 2.4$ & 44.01 \\
\hline & 9 & $166_{-10}^{+11}$ & 352.62 & 31 & $13.8_{-2.0}^{+2.3}$ & 44.01 \\
\hline & 10 & $158 \pm 9$ & 352.62 & 32 & $15.3+2.0$ & 44.01 \\
\hline & 11 & $137 \pm 8$ & 352.62 & 33 & $19.5 \pm 2.2$ & 44.01 \\
\hline & 12 & $150 \pm 8$ & 352.62 & 34 & $17.0 \pm 2.0$ & 44.01 \\
\hline & 13 & $141+9$ & 352.62 & 35 & $20.4_{-2.3}^{+2.7}$ & 44.01 \\
\hline & 14 & $137_{-9}^{+10}$ & 352.62 & 36 & $4.28+0.48$ & 9.38 \\
\hline & 15 & $87.8 \pm 2.6$ & 220.59 & 37 & $4.78+1.45$ & 9.38 \\
\hline & 16 & $90.7+7.2$ & 220.59 & 38 & $6.97+1.56$ & 9.38 \\
\hline & 17 & $\begin{array}{l}92.1+6.7 \\
-6.4\end{array}$ & 220.59 & 39 & $\begin{array}{l}5.82+1.22 \\
-0.98\end{array}$ & 9.38 \\
\hline & 18 & $90.5+5.8$ & 220.59 & 40 & $\begin{array}{l}5.58+1.19 \\
-0.95\end{array}$ & 9.38 \\
\hline & 19 & $99.8+5.96$ & 220.59 & 41 & $3.70+1.14$ & 9.38 \\
\hline & 20 & $90.3+6.4$ & 220.59 & 42 & $\begin{array}{l}3.93+1.27 \\
-0.93\end{array}$ & 9.38 \\
\hline & 21 & $88.5+7.0$ & 220.59 & 43 & $0.341+0.077$ & 0.669 \\
\hline \multirow[t]{17}{*}{ SK-II $[20]$} & 0 & $43.7_{-5.1}^{+5.2}$ & 110.7 & & & \\
\hline & 1 & $\begin{array}{r}36.4+5.1 \\
-4.9\end{array}$ & 97.63 & 2 & $43.6+5.2+5$ & 97.63 \\
\hline & 3 & $34.4+3.5$ & 84.65 & 4 & $\begin{array}{l}35.5+3.5 \\
-3.4\end{array}$ & 84.65 \\
\hline & 5 & $27.0+2.8$ & 72.45 & 6 & $33.0+2.8$ & 72.45 \\
\hline & 7 & $23.9+2.3$ & 60.54 & 8 & $25.0+2.3$ & 60.54 \\
\hline & 9 & $20.7_{-1.9}^{+2.0}$ & 49.61 & 10 & $23.3_{-1.9}^{+2.0}$ & 49.61 \\
\hline & 11 & $15.4+1.7$ & 40.16 & 12 & $17.6_{-1.6}^{+1.7}$ & 40.16 \\
\hline & 13 & $13.5+1.5$ & 31.69 & 14 & $14.2_{-1.4}^{+1.5}$ & 31.69 \\
\hline & 15 & $11.3_{-1.2}^{+1.3}$ & 24.99 & 16 & $9.4+1.2$ & 24.99 \\
\hline & 17 & $7.11+0.00$ & 19.29 & 18 & $8.96+0.94$ & 19.29 \\
\hline & 19 & $6.82_{-0.84}^{+0.94}$ & 14.52 & 20 & $5.79+0.86$ & 14.52 \\
\hline & 21 & $4.18+0.73$ & 10.86 & 22 & $\begin{array}{l}3.97+0.70 \\
-0.61\end{array}$ & 10.86 \\
\hline & 23 & $\begin{array}{r}2.95+0.62 \\
-0.53\end{array}$ & 7.84 & 24 & $\begin{array}{l}3.66+0.61 \\
-0.53\end{array}$ & 7.84 \\
\hline & 25 & $2.95+0.57$ & 5.70 & 26 & $\begin{array}{l}1.59+0.44 \\
-0.35\end{array}$ & 5.70 \\
\hline & 27 & $2.99+0.60$ & 6.87 & 28 & $2.58+0.53$ & 6.87 \\
\hline & 29 & $1.37+0.42$ & 3.41 & 30 & $2.08+0.45$ & 3.41 \\
\hline & 31 & $1.11+0.37$ & 2.56 & 32 & $1.60+0.40$ & 2.56 \\
\hline
\end{tabular}

Table C.1: Data from other solar neutrino experiments. Normalization rates are rescaled to the $\mathrm{BS} 05(\mathrm{OP})$ predictions. In total there are $3+44+33=80$ rates. See text for details. 


\section{Systematic Uncertainties}

The rates in Table C.1 come with statistical uncertainties. Systematic uncertainties taken from the publications were added to the statistical uncertainties following the recommended prescription from each collaboration. For example, the SK energy systematic uncertainties were obtained by smearing the detector response parameters using the energy scale and resolution parameter shifts provided in $[19,20]$. The energy systematic uncertainties were assumed to be fully correlated in the energy bins they affected when the error matrix of Equation (6.7) was built.

The cross-section uncertainties applied to the Homestake and Gallium experiments were calculated separately for $\mathrm{HE}\left({ }^{8} \mathrm{~B}\right.$ and hep) and LE neutrinos (six other sources) and combined quadratically:

$$
u_{n}^{2}(\mathrm{cross})=\left(\sum_{i \in \mathrm{LE}} \Delta F_{n}^{\mathrm{th}}\right)^{2}+\left(\sum_{i \in \mathrm{HE}} \Delta F_{n}^{\mathrm{th}}\right)^{2}
$$

and added to the total uncertainties from Table C.1, following the procedure in [6].

\section{C.2 More on Neutrino Oscillation Parameters}

This section refers to Chapter 7. Subsection C.2.1 discusses the treatment of the KamLAND data. Subsection C.2.2 gives the decomposition of the uncertainties of the BS05(OP) model. Subsection C.2.3 gives the $2 \nu$ and $3 \nu$ model contours from the individual solar neutrino experiments. Finally Subsection C.2.4 explains how the results of the survival probability fits can be interpreted as oscillation parameters. 


\section{C.2.1 KamLAND}

The KamLAND survival probabilities of $\bar{\nu}_{e}$ 's were calculated using the vacuum formula of Equation (1.9), neglecting the small matter effects in the Earth crust. The survival probabilities were determined from the distances $L_{i}$ between a list of reactors $i$ and the KamLAND detector. The $2 \nu$ probabilities, $P_{e e}^{2 \nu}$, were calculated with the vacuum formula of Equation (1.9). The $3 \nu$ probabilities were obtained with [151]:

$$
P_{e e}^{3 \nu}=\cos ^{4} \theta_{13} P_{e e}^{2 \nu}+\sin ^{4} \theta_{13} .
$$

The KamLAND Collaboration provided the results of Fig. 1 in [42]. The 16 positron-energy rates were built by subtracting the measured backgrounds from the total rates. The unoscillated rates were used to calibrate the predicted rates in the analytic model. The energy resolution of $6.5 \% / \sqrt{E}$ was applied in the response function. The main systematic uncertainties were the reactor rates (4\%) and energy scale $(2 \%)$. With the above, the $2 \nu$ analysis gave results that were comparable to Fig. 2 in [42]. The KamLAND Collaboration has not yet published analyses in which they include detailed contour plots with $\theta_{13}$.

\section{C.2.2 SSM Partial Derivatives}

The SSM partial derivatives are a set of model systematic uncertainties affecting the predicted flux separately for each neutrino type. They correspond to uncertainties in the model input parameters propagated to the fluxes. In the BS05(OP) calculation, there are 20 input parameters, listed in Table C.2. The first column gives the name of the parameters, $p$, the second the uncertainty on the parameter, and the remaining 


\begin{tabular}{|cc|ccccccccc|}
\hline \hline & & 0 & 1 & 2 & 3 & 4 & 5 & 6 & 7 \\
\hline$p$ & $\Delta p$ & pp & pep & hep & ${ }^{7} \mathrm{Be}$ & ${ }^{8} \mathrm{~B}$ & ${ }^{13} \mathrm{~N}$ & ${ }^{15} \mathrm{O}$ & ${ }^{17} \mathrm{~F}$ \\
\hline$S_{11}$ & 1.000 & +0.001 & -0.001 & -0.000 & -0.004 & -0.010 & -0.010 & -0.011 & -0.011 \\
$S_{33}$ & 1.000 & +0.002 & +0.003 & -0.024 & -0.023 & -0.021 & +0.001 & +0.001 & +0.001 \\
$S_{34}$ & 1.000 & -0.005 & -0.007 & -0.007 & +0.080 & +0.075 & -0.004 & -0.004 & -0.004 \\
$S_{114}$ & 1.000 & -0.002 & -0.002 & -0.001 & +0.000 & +0.001 & +0.079 & +0.095 & +0.001 \\
$S_{17}$ & 1.000 & +0.000 & +0.000 & +0.000 & +0.000 & +0.038 & +0.000 & +0.000 & +0.000 \\
$S_{\text {hep }}$ & 1.000 & +0.000 & +0.000 & +0.151 & +0.000 & +0.000 & +0.000 & +0.000 & +0.000 \\
$L_{\odot}$ & 1.000 & +0.003 & +0.003 & +0.000 & +0.014 & +0.028 & +0.021 & +0.024 & +0.026 \\
$Z / X$ & - & -0.007 & -0.013 & -0.020 & +0.053 & +0.116 & +0.262 & +0.262 & +0.483 \\
$\mathrm{Age}$ & 1.000 & -0.000 & +0.000 & -0.000 & +0.003 & +0.006 & +0.004 & +0.006 & +0.006 \\
$\mathrm{Op}$. & 1.000 & +0.003 & +0.005 & +0.011 & -0.028 & -0.052 & -0.033 & -0.041 & -0.043 \\
$\mathrm{Diff}$ & 1.000 & +0.003 & +0.004 & +0.007 & -0.018 & -0.040 & -0.051 & -0.055 & -0.057 \\
$\mathrm{Be} 7 \mathrm{e}$ & 1.000 & +0.000 & +0.000 & +0.000 & +0.000 & -0.020 & +0.000 & +0.000 & +0.000 \\
$\mathrm{C}$ & 0.297 & -0.014 & -0.025 & -0.015 & -0.002 & +0.030 & +0.845 & +0.826 & +0.033 \\
$\mathrm{~N}$ & 0.320 & -0.003 & -0.006 & -0.004 & +0.002 & +0.011 & +0.181 & +0.209 & +0.010 \\
$\mathrm{O}$ & 0.387 & -0.006 & -0.011 & -0.023 & +0.052 & +0.121 & +0.079 & +0.093 & +1.102 \\
$\mathrm{Ne}$ & 0.539 & -0.005 & -0.005 & -0.017 & +0.049 & +0.096 & +0.057 & +0.068 & +0.076 \\
$\mathrm{Mg}$ & 0.115 & -0.005 & -0.005 & -0.018 & +0.051 & +0.096 & +0.060 & +0.070 & +0.078 \\
$\mathrm{Si}$ & 0.115 & -0.011 & -0.014 & -0.037 & +0.104 & +0.194 & +0.128 & +0.150 & +0.164 \\
$\mathrm{~S}$ & 0.092 & -0.008 & -0.017 & -0.028 & +0.074 & +0.137 & +0.094 & +0.109 & +0.120 \\
$\mathrm{Ar}$ & 0.496 & -0.002 & -0.006 & -0.007 & +0.018 & +0.034 & +0.024 & +0.028 & +0.031 \\
$\mathrm{Fe}$ & 0.115 & -0.023 & -0.065 & -0.069 & +0.209 & +0.515 & +0.342 & +0.401 & +0.444 \\
\hline \hline
\end{tabular}

Table C.2: Systematic uncertainties of the BS05(OP) SSM in the form of partial derivatives. An uncertainty $\Delta p=1$ means the derivative already includes the weight of the uncertainty. Values without enough digits can be neglected.

columns the partial derivatives for each neutrino flux component.

The details of the source and impact of each parameter can be found in [6]. The $S$ terms correspond to the low-energy cross-section uncertainties that impact the nuclear rates of each reaction directly: $S_{11}, S_{33}, S_{34}, S_{114}, S_{17}$, and $S_{\text {hep }}$ correspond to $p-p$, ${ }^{3} \mathrm{He}-{ }^{3} \mathrm{He},{ }^{3} \mathrm{He}\left({ }^{4} \mathrm{He}, \gamma\right){ }^{7} \mathrm{Be},{ }^{14} \mathrm{~N}(p, \gamma){ }^{15} \mathrm{O},{ }^{7} \mathrm{Be}(p, \gamma){ }^{8} \mathrm{~B}$, and hep reactions, respectively. Then follow the Sun luminosity $L_{\odot}$, the heavy metallicity factor $Z / X$, the age of the Sun (Age), the opacity of the Sun (Op.), the diffusion model uncertainty (Diff.), 
the theoretical rate of the electron capture reaction ${ }^{7} \mathrm{Be}\left(e^{-}, \nu\right)^{7} \mathrm{Li}$ (denoted $\mathrm{Be} 7 \mathrm{e}$ ), and the list of heavy element compositions. In the BS05(OP) model, the systematic uncertainty $Z / X$ is canceled on purpose and replaced by the decomposition of heavy element uncertainties (from $\mathrm{C}$ to $\mathrm{Fe}$ ).

The partial derivatives, often referred to as logarithmic derivatives $\alpha_{k i}[4]$, are then applied to each decomposed terms of the fractional rates to build the error matrix part due to the SSM:

$$
\begin{aligned}
F_{n}^{\text {th }} & =\sum_{i}^{8} F_{n}^{i} \\
\sigma_{\mathrm{SSM}}^{2} & =\sum_{i}^{8} F_{n}^{i} \sum_{j}^{8} F_{n}^{j} \sum_{k}^{20}\left(\Delta p_{k}\right)^{2} \alpha_{k i} \alpha_{k j} .
\end{aligned}
$$

\section{C.2.3 Contours from Individual Experiments}

This subsection provides individual contours in the $\left(\tan ^{2} \theta_{12}, \Delta m_{21}^{2}\right)$ plane obtained from the external data listed in Subsection C.1.3. Figures C.4 and C.5 show the confidence regions projected into the $\left(\tan ^{2} \theta_{12}, \Delta m_{21}^{2}\right)$ plane obtained from the LE (Homestake, Gallium, and Borexino) and HE (SK) experiments. In each plot, the comparison between the $2 \nu$ and the $3 \nu$ models is shown. The nomenclature SSMFloat means that the partial derivatives of the SSM were left out of the fit, and that the fluxes of the model were free to vary but were constrained with penalty terms corresponding to their $1 \sigma$ uncertainties.

From Figure C.4d, the conclusion is that the low-energy experiments constrain well the LMA and LOW regions, but are also very sensitive to low $\Delta m_{21}^{2}$ solutions, or the vacuum regime. From Figure C.5c, one can see that the SK ES data cannot 
constrain the space well without knowing the total neutrino flux provided by SNO. Nevertheless, once all low-energy and SK data are combined as in Figure C.5d, the space is constrained to the LMA and LOW regions. One interesting observation is that the LMA and LOW region, with the effect of $\theta_{13}$, prefer different sides of the $\tan ^{2} \theta_{12}$ boundary. With the addition of the SNO data the choice is made rather towards the LMA region, with $\tan ^{2} \theta_{12}<1$.

\section{C.2.4 Recipe to Interpret Survival Probabilities}

The subsection explains how to treat the survival probability fits shown in Chapter 6 in the extraction of the oscillation parameters. The method translates the $P_{e e}\left(E_{\nu}\right)$ function into mixing parameters, independently of the survival probability parameterization. The following describes how this method was used to obtain identical oscillation parameters and constraints as given in Chapter 7 .

The best-fit survival probability and flux scale parameters, $\vec{\mu}_{i}$, and covariance matrix, $V_{i j}$, were used to generate a large number (typically $10^{5}$ ) of random values of the parameters, $x_{i}$. The MC generation of the correlated parameters was achieved following the method outlined in Monte Carlo Techniques in [2]. For each parameter set $k$, the survival probability and flux scales $x_{i}^{k}$ were used as fixed parameters to calculate the model predicted rates $F_{n}^{\text {th }}(k)$, from which the value of the observables, systematic errors, and error matrix elements from Equations (6.6) and (6.7) were evaluated. After a large number of evaluations, the mean and spread of the $F_{n}^{\text {th }}$ distributions were taken as the effective value and total uncertainty, respectively, that were compatible with the survival probability $1 \sigma$-error band. The means $\vec{\mu}_{i}$ were known from the nominal survival probability fit, therefore the estimators for variance 


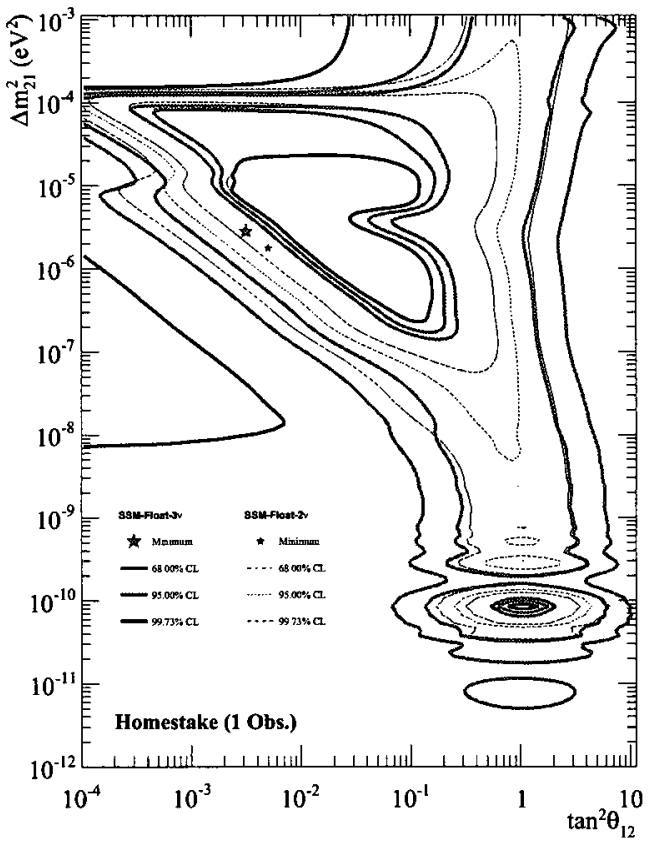

(a) Homestake.

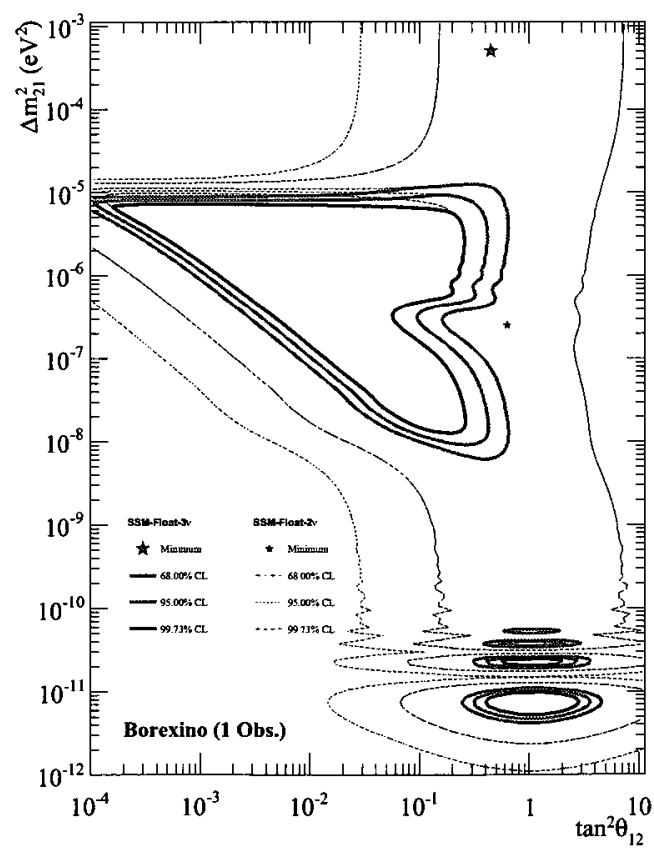

(c) Borexino.

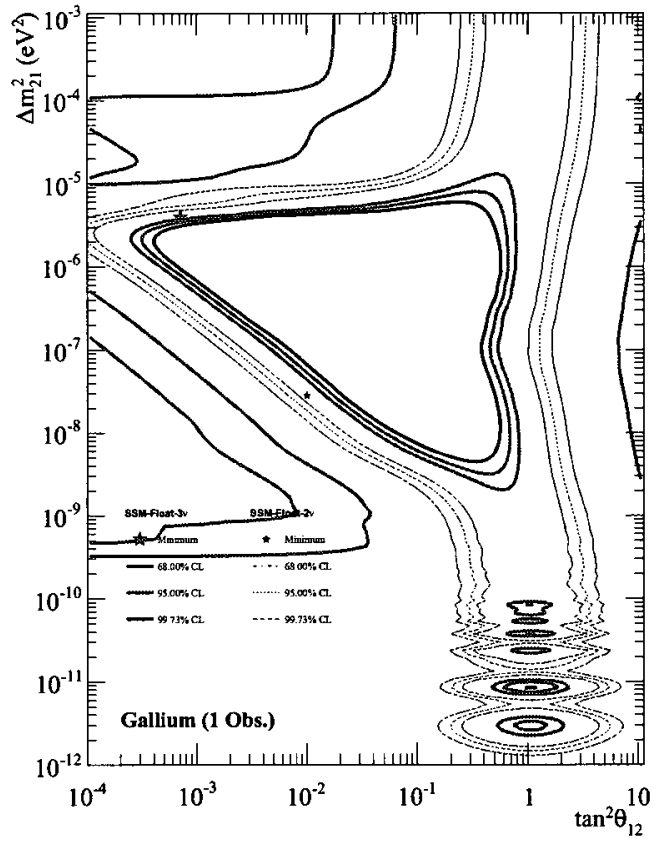

(b) Gallium.

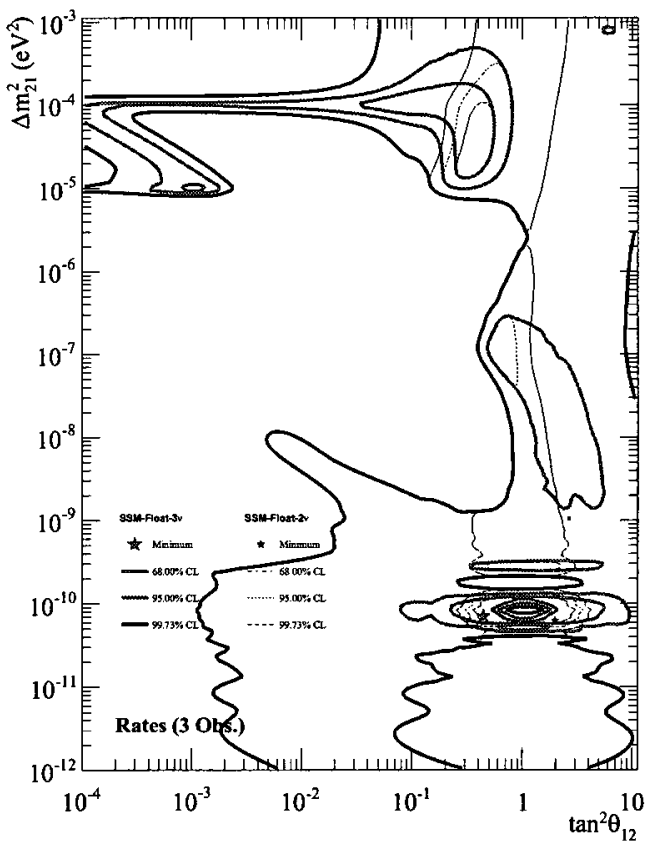

(d) Homestake+Gallium+Borexino.

Figure C.4: Confidence regions of the oscillation parameters obtained with the low-energy experiments. The $\chi^{2}$ was minimized with respect to the undisplayed parameters. 


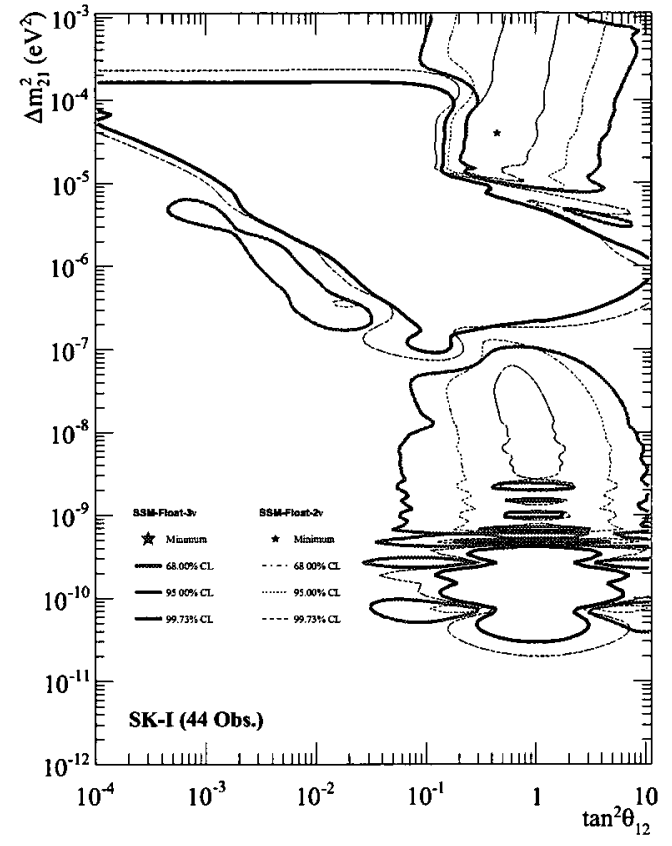

(a) SK-I.

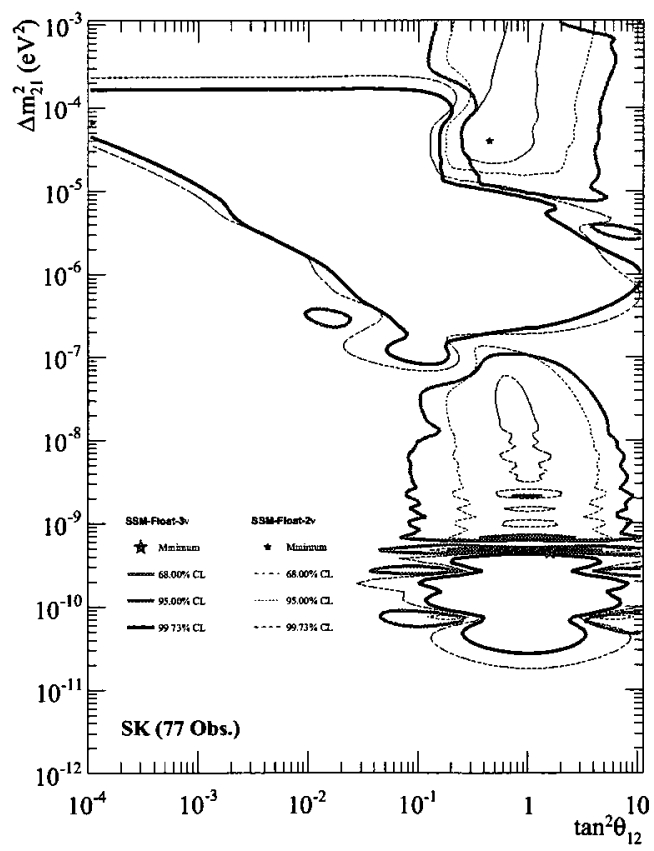

(c) SK $=$ SK-I + SK-II.

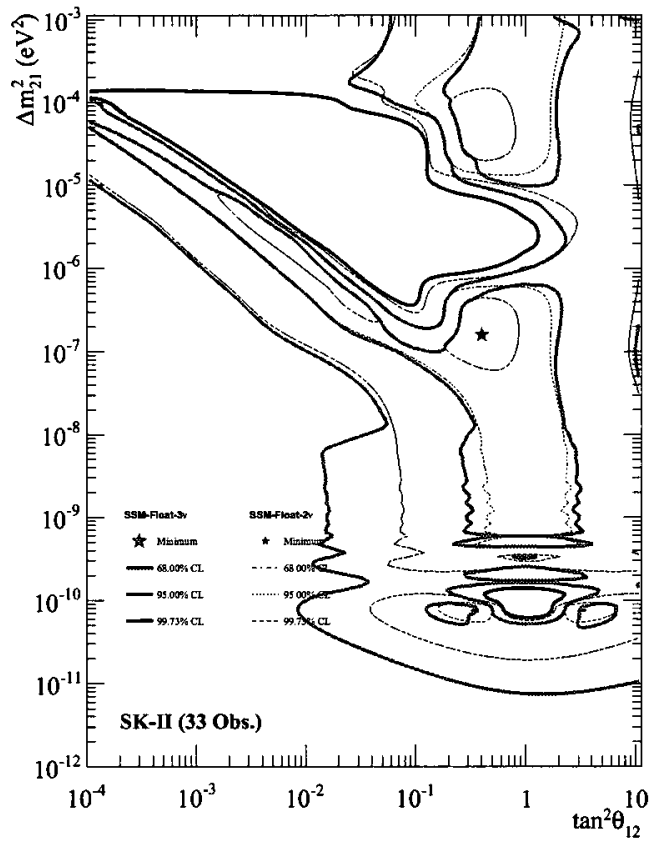

(b) SK-II.

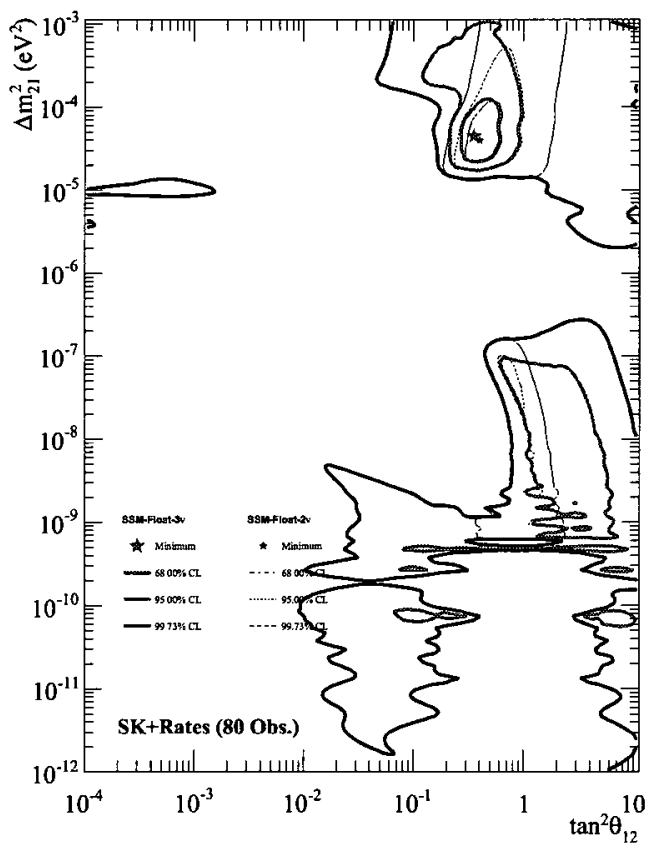

(d) Homestake+Gallium+Borexino+SK.

Figure C.5: Confidence regions of the oscillation parameters obtained with the low-energy and Super-Kamiokande experiments. The $\chi^{2}$ was minimized with respect to the undisplayed parameters. 
and covariance elements were:

$$
\begin{aligned}
& S_{i}^{2} \equiv S^{2}\left(x_{i}\right)=\frac{1}{n} \sum_{k=1}^{n}\left(x_{i}^{k}-\mu_{i}\right)^{2} \\
& \hat{V}_{i j}=\frac{1}{n} \sum_{k=1}^{n}\left(\left(x_{i}^{k}-\mu_{i}\right)\left(x_{j}^{k}-\mu_{j}\right)\right) .
\end{aligned}
$$

The elements of the error matrix were used to calculated the correlations between observables due to the survival probability. Finally the effective set of rates, uncertainties, and correlation matrix were tested as a regular data set using the grid-scan method outlined in Chapter 7, and the oscillation parameters were recovered with the same accuracy.

After the final extraction of the SNO signals is performed with the combined fit of the three phases, one should be able to test if the survival probability fit performed directly in the SigEx code results in smaller constraints in the oscillation parameters, due to the constrained nature of the SigEx formalism. 


\section{Bibliography}

[1] K. Winter, Neutrino Physics, Second ed. (Cambridge University Press, 2000).

[2] Particle Data Group, C. Amsler et al., Phys. Lett. B667, 1 (2008).

[3] Super-Kamiokande Collaboration, Y. Fukuda et al., Phys. Rev. Lett. 81, 1562 (1998).

[4] J. N. Bahcall, Neutrino Astrophysics (Cambridge University Press, 1989).

[5] J. N. Bahcall et al., Astrophys. J. 621, L85 (2005).

[6] J. N. Bahcall et al., Astrophys. J. Suppl. 165, 400 (2006).

[7] L. C. Stonehill, J. A. Formaggio, and R. G. H. Robertson, Phys. Rev. C69, 015801 (2004).

[8] Z. Maki et al., Prog. Theor. Phys. 28, 870 (1962).

[9] V. Gribov and B. Pontecorvo, Phys. Lett. B28, 493 (1969).

[10] L. Wolfenstein, Phys. Rev. D17, 2369 (1978).

[11] S. P. Mikheev and A. Y. Smirnov, Sov. J. Nucl. Phys. 42, 913 (1985).

[12] P. C. de Holanda and A. Y. Smirnov, Phys. Rev. D69, 113002 (2004).

[13] B. T. Cleveland et al., Astrophys. J. 496, 505 (1998).

[14] GNO Collaboration, M. Altmann et al., Phys. Lett. B616, 174 (2005).

[15] SAGE Collaboration, J. N. Abdurashitov et al., J. Exp. Theor. Phys. 95, 181 (2002).

[16] SAGE Collaboration, J. N. Abdurashitov et al., Phys. Rev. C80, 015807 (2009). 
[17] Super-Kamiokande Collaboration, S. Fukuda et al., Nucl. Instrum. Meth. A501, 418 (2003).

[18] Kamiokande Collaboration, Y. Fukuda et al., Phys. Rev. Lett. 77, 1683 (1996).

[19] Super-Kamiokande Collaboration, J. Hosaka et al., Phys. Rev. D73, 112001 (2006).

[20] Super-Kamiokande Collaboration, J. P. Cravens et al., Phys. Rev. D78, 032002 (2008).

[21] SNO Collaboration, J. Boger et al., Nucl. Instrum. Meth. A449, 172 (2000).

[22] G. T. Ewan et al., Sudbury Neutrino Observatory Proposal, SNO-87-12 (1987).

[23] H. H. Chen, Phys. Rev. Lett. 55, 1534 (1985).

[24] SNO Collaboration, Q. R. Ahmad et al., Phys. Rev. Lett. 87, 071301 (2001).

[25] SNO Collaboration, Q. R. Ahmad et al., Phys. Rev. Lett. 89, 011301 (2002).

[26] SNO Collaboration, B. Aharmim et al., Phys. Rev. C75, 045502 (2007).

[27] SNO Collaboration, B. Aharmim et al., Phys. Rev. Lett. 101, 111301 (2008).

[28] SNO Collaboration, Q. R. Ahmad et al., Phys. Rev. Lett. 89, 011302 (2002).

[29] SNO Collaboration, S. N. Ahmed et al., Phys. Rev. Lett. 92, 181301 (2004).

[30] SNO Collaboration, Q. R. Ahmad et al., Phys. Rev. C72, 055502 (2005).

[31] SNO Collaboration, B. Aharmim et al., Astrophys. J. 653, 1545 (2006).

[32] SNO Collaboration, B. Aharmim et al., Phys. Rev. D72, 052010 (2005).

[33] L. Heelan, A Search for Periodic Time Variations in the Solar Neutrino Data from the Sudbury Neutrino Observatory, Master's thesis, Carleton University, Ottawa, Canada (2005). 
[34] SNO Collaboration, B. Aharmim et al., Accepted for publication in Phys. Rev. D (2009).

[35] SNO Collaboration, B. Aharmim et al., Low Energy Threshold Analysis: to be published (2009).

[36] SNO Collaboration, B. Aharmim et al., The Three-Phase Analysis: to be published (2010).

[37] Borexino Collaboration, G. Alimonti et al., Submitted to Nucl. Instrum. Meth., arXiv.0806.2400 (2008).

[38] Borexino Collaboration, C. Arpesella et al., Phys. Lett. B658, 101 (2008).

[39] Borexino Collaboration, C. Arpesella et al., Phys. Rev. Lett. 101, 091302 (2008).

[40] KamLAND Collaboration, K. Eguchi et al., Phys. Rev. Lett. 90, 021802 (2003).

[41] KamLAND Collaboration, T. Araki et al., Phys. Rev. Lett. 94, 081801 (2005).

[42] KamLAND Collaboration, S. Abe et al., Phys. Rev. Lett. 100, 221803 (2008).

[43] J. R. Klein, J. Phys. Conf. Ser. 136, 022004 (2008).

[44] CHOOZ Collaboration, M. Apollonio et al., Phys. Lett. B466, 415 (1999).

[45] F. Ardellier et al., hep-ex/0405032 (2004).

[46] K2K Collaboration, E. Aliu et al., Phys. Rev. Lett. 94, 081802 (2005).

[47] MINOS Collaboration, P. Adamson et al., Phys. Rev. Lett. 101, 221804 (2008).

[48] SNO+ Collaboration, http://snoplus.phy.queensu.ca (2009).

[49] EXO Collaboration, http://www-project.slac.stanford.edu/exo (2009).

[50] J. F. Amsbaugh et al., Nucl. Instrum. Meth. A579, 1054 (2007). 
[51] L. C. Stonehill, Deployment and Background Characterization of the Sudbury Neutrino Observatory Neutral Current Detectors, PhD thesis, University of Washington, Seattle, USA (2005).

[52] J. R. Klein et al., The SNO Trigger System, SNO Technical Report, SNO-STR97-036 (1997).

[53] S. Biller et al., SNO Electronics Calibration Constants, SNO Technical Report, SNO-STR-2001-005 (2001).

[54] P. Skensved, ECA, PCA and Laserball Reconstruction, SNO Technical Report, SNO-STR-2000-007 (2000).

[55] B. A. Moffat et al., Nucl. Instrum. Meth. A554, 255 (2005).

[56] B. A. Moffat, The Optical Calibration of the Sudbury Neutrino Observatory, $\mathrm{PhD}$ thesis, Queen's University, Kingston, Canada (2001).

[57] M. R. Dragowsky et al., Nucl. Instrum. Meth. A481, 284 (2002).

[58] R. F. MacLellan, The Energy Calibration for the Solar Neutrino Analysis of All Three Phases of the Sudbury Neutrino Observatory, PhD thesis, Queen's University, Kingston, Canada (2009).

[59] N. J. Tagg et al., Nucl. Instrum. Meth. A489, 178 (2002).

[60] A. W. P. Poon et al., Nucl. Instrum. Meth. A452, 115 (2000).

[61] M. S. Kos, Low Energy Solar Neutrino Analysis of the Salt Phase of the Sudbury Neutrino Observatory, PhD thesis, Queen's University, Kingston, Canada (2007).

[62] S. Brice et al., First Generation Acrylic Encapsulated U/Th Sources, SNO Technical Report, SNO-STR-99-023 (2000).

[63] M. Thomson, Radon Spike Source Calibration of the SNO Detector, Master's thesis, Queen's University, Kingston, Canada (2005). 
[64] G. A. Cox-Mobrand, Data Integrity and Electronic Calibrations for the Neutral Current Detector Phase Measurement of the ${ }^{8} B$ Solar Neutrino Flux at the Sudbury Neutrino Observatory, PhD thesis, University of Washington, Seattle, USA (2008).

[65] J. C. Loach, Measurement of the Flux of ${ }^{8} B$ Solar Neutrinos at the Sudbury Neutrino Observatory, PhD thesis, University of Oxford, Oxford, UK (2008).

[66] C. Howard, Injection of a Uniform ${ }^{24} \mathrm{Na}$ Radioactive Source into the Sudbury Neutrino Observatory, Master's thesis, Queen's University, Kingston, Canada (2006).

[67] T. C. Andersen et al., Nucl. Instrum. Meth. A501, 386 (2003).

[68] T. C. Andersen et al., Nucl. Instrum. Meth. A501, 399417 (2003).

[69] B. Aharmim et al., Nucl. Instrum. Meth. A604, 531 (2009).

[70] SNO Collaboration, The NCD Analysis Group, The NCD Unidoc v8, SNO Internal Note, MANN-5D54GA (2002).

[71] SNO Collaboration, The SNOMAN User Manual, Internal reference manual (2009).

[72] CERN Program Library Writeups Q100/101, CERN, Geneva, Switzerland, http://cernlib.web.cern.ch/cernlib (1995).

[73] CERN Program Library Long Writeup Q180, CERN, Geneva, Switzerland, http://cernlib.web.cern.ch/cernlib (1995).

[74] W. R. Nelson, H. Hirayama, and D. W. O. Rogers, The EGS4 Code System, Stanford Linear Accelerator Center Report, SLAC-265 (1985).

[75] M. D. Lay, Creation and Detection of Čerenkov Light in the Sudbury Neutrino Observatory, PhD thesis, Oxford University, Oxford, UK (1994).

[76] J. Briesmeister et al., MCNP4A A General Monte Carlo N-Particle Transport Code, Los Alamos National Laboratory, LA-12625-M (1993). 
[77] G. Ingelman et al., Comp. Phys. Comm. 101, 108 (1997).

[78] P. Aarnio et al., FLUKA: hadronic benchmarks and applications, Proc. Int. Conference on Monte Carlo Simulation in High Energy and Nuclear Physics (MC93), http://www.fluka.org (1993).

[79] N. S. Oblath et al., NCD MC Readiness Report for the First NCD Paper, SNO Internal Note, MANN-79662C (2008).

[80] G. D. Orebi-Gann, An Improved Measurement of the ${ }^{8} B$ Solar Neutrino Energy Spectrum at the Sudbury Neutrino Observatory, $\mathrm{PhD}$ thesis, University of Oxford, Oxford, UK (2008).

[81] D. R. Grant, Optical Calibration of the Sudbury Neutrino Observatory and Determination of the ${ }^{8} B$ Solar Neutrino Flux in the Salt Phase, PhD thesis, Carleton University, Ottawa, Canada (2004).

[82] C. V. Ouellet, A Study of Relative Efficiencies of Photomultiplier Tubes in the Sudbury Neutrino Observatory, Master's thesis, Queen's University, Kingston, Canada (2003).

[83] M. Dunford, Measurement of the ${ }^{8} B$ Solar Neutrino Energy Spectrum at the Sudbury Neutrino Observatory, PhD thesis, University of Pennsylvania, Philadelphia, USA (2006).

[84] O. Simard and J. C. Maneira, Optical Constants Extraction using the PMT Occupancy Fit Method, SNO Internal Note, MANN-6HGQGQ (2005).

[85] J. C. Maneira, PMT variability with incidence angle in salt optics, SNO Internal Note, MANN-5SUSDE (2003).

[86] J. C. Maneira, Update on PMT Efficiencies, SNO Internal Note, MANN6G7L7K (2005).

[87] O. Simard, An Enhanced Measurement of the Angular Response of the Photomultiplier Tubes at the Sudbury Neutrino Observatory, Master's thesis, Carleton University, Ottawa, Canada (2005). 
[88] O. Simard, Changes to the Optics Scan Plan during the NCD Phase, SNO Internal Note, MANN-6A7TY3 (2005).

[89] O. Simard, Low Statistics Effects on Optical Constants Fits, SNO Internal Note, MANN-6HWSMQ (2005).

[90] N. F. de Barros and J. C. Maneira, Tests on the Optical Calibration of batch 3 reflectors, SNO Internal Note, MANN-6R2PH6 (2006).

[91] J. R. Klein, Realistic Simulated Charge Spectrum for SNO and the Prediction of Tube-by-tube Efficiencies, SNO Internal Note, MANN-6EX424 (2005).

[92] J. R. Klein, Simulating Threshold- and Gain-Dependent Channel Efficiencies and the Solution to the Multi-pe Efficiency Problem, SNO Internal Note, MANN-6FQPK6 (2005).

[93] J. C. Maneira, Laserball Deployment Plan Proposal for NCD Phase, SNO Internal Note, MANN-5W4NN4 (2004).

[94] C. Kraus, Calibration Schedules and Run Plans, SNO Internal Note, http://www.sno.phy.queensu.ca/ tine/private/calschedule_draft.html (2008).

[95] A. Hallin, Fitting the Laserball Positions without PCA information, SNO Internal Note, MANN-6N4G4Z (2006).

[96] N. Oblath, Multipoint PCA Description, SNO Internal Note, LANL-6663YV (2004).

[97] O. Simard et al., Optical Calibrations in the NCD Phase, SNO Internal Note, MANN-6YSTJB (2007).

[98] K. Boudjemline and A. Hallin, PCA Offset Correction for the NCD Phase, SNO Internal Note, MANN-6Z2KX4 (2007).

[99] H. Robertson, Private Communication (2007). 
[100] O. Simard, Verifying the NCD Positions using Laserball Data, SNO Internal Note, MANN-6LM526 (2006).

[101] G. Cowan, Statistical Data Analysis (Oxford University Press, 1998).

[102] R. Brun et al., ROOT - An Object Oriented Data Analysis Framework, http://root.cern.ch (2009).

[103] P.-L. Drouin, FTN: A Time Fitter for the NCD Phase, SNO Internal Note, Version 7.0, MANN-78VP3B (2007).

[104] J. Radigan and C. Kraus, Measurements of the NCD Reflectivity, SNO Internal Note, LANL-6EKGLB (2005).

[105] R. S. Dosanjh, End-of-Salt Optics Extraction, SNO Internal Note, Version 1.2, MANN-5VTMZT (2004).

[106] W. H. Press et al., Numerical Recipes in C++: The Art of Scientific Computing, Second Edition (Cambridge University Press, 2002).

[107] C. Waltham, Concentrator Reflectometry, SNO Internal Note, MANN-59339D (2002).

[108] O. Simard, A New Laserball Model for the Optics in the NCD Phase, SNO Internal Note, MANN-6RTNUE (2006).

[109] R. F. MacLellan, RSP for the NCD Phase, SNO Internal Note, MANN78WMNH (2007).

[110] M. G. Boulay, Direct Evidence for Weak Flavor Mixing with the Sudbury Neutrino Observatory, PhD thesis, Queen's University, Kingston, Canada (2001).

[111] R. F. MacLellan, Private Communication (2007).

[112] O. Simard, Checks on Current NCD-Phase PMT Efficiencies, SNO Internal Note, MANN-6UG7QZ (2006). 
[113] S. R. Seibert, A Low Energy Measurement of the ${ }^{8} B$ Solar Neutrino Spectrum at the Sudbury Neutrino Observatory, $\mathrm{PhD}$ thesis, University of Texas, Austin, USA (2008).

[114] SNO Collaboration, The LETA Working Group, The Low Energy Threshold Analysis Unidoc, SNO Internal Note (2009).

[115] M. S. Neubauer, Evidence for Electron Neutrino Flavor Change Through Measurement of the ${ }^{8} B$ Solar Neutrino Flux at the Sudbury Neutrino Observatory, PhD thesis, University of Pennsylvania, Philadelphia, USA (2001).

[116] J. Wilson, A Measurement of the ${ }^{8} B$ Solar Neutrino Energy Spectrum at the Sudbury Neutrino Observatory, PhD thesis, Oxford University, Oxford, UK (2004).

[117] P.-L. Drouin, Thesis in preparation, PhD thesis, Carleton University, Ottawa, Canada (2010).

[118] CERN Program Library Writeup D506, CERN, Geneva, Switzerland, http://cernlib.web. cern. ch/cernlib (1998).

[119] O. Simard, Physics Interpretation - The Solar Standard Model, SNO Internal Note, MANN-7B5445-leta00 (2008).

[120] O. Simard, Physics Interpretation - The Survival Probability at the Surface of the Sun, SNO Internal Note, MANN-7B5445-leta01 and leta02 (2008).

[121] O. Simard, Physics Interpretation - The ${ }^{8} \mathrm{~B}$ Flux Scale, SNO Internal Note, MANN-7B5445-leta03 (2008).

[122] O. Simard, Physics Interpretation - The Survival Probability Formulae, SNO Internal Note, MANN-7B5445-leta04 and leta05 (2008).

[123] O. Simard, Physics Interpretation - The Analytic Response Functions, SNO Internal Note, MANN-7B5445-leta06 (2008).

[124] O. Simard, Physics Interpretation - The Day-Night Asymmetry, SNO Internal Note, MANN-7B5445-leta07 (2008). 
[125] O. Simard, Physics Interpretation - The Survival Probability Dependence on $\Delta m_{31}^{2}$, SNO Internal Note, MANN-7B5445-leta08 (2008).

[126] O. Simard, Physics Interpretation - The Survival Probability Precision, SNO Internal Note, MANN-7B5445-leta09 (2008).

[127] O. Simard, Physics Interpretation - The Survival Probability Fit, SNO Internal Note, MANN-7B5445-leta10 (2008).

[128] P.-L. Drouin, SNO Likelihood Function and CC and ES Probability Density Functions, SNO Internal Note, MANN-7SA5NN (2009).

[129] C. E. Ortiz et al., Phys. Rev. Lett. 85, 2909 (2000).

[130] W. T. Winter et al., Phys. Rev. C73, 025503 (2006).

[131] M. Butler, J.-W. Chen, and X. Kong, Phys. Rev. C63, 035501 (2001).

[132] A. Kurylov et al., Phys. Rev. C65, 055501 (2002).

[133] S. Nakamura et al., Nucl. Phys. A707, 561 (2002).

[134] B. Mosconi et al., Phys. Rev. C75, 044610 (2007).

[135] J. N. Bahcall et al., Phys. Rev. D51, 6146 (1995).

[136] M. Chen and A. W. P. Poon, NC cross sections, SNO Internal Note, MANN7EFAAX (2008).

[137] Super-Kamiokande, M. B. Smy et al., Phys. Rev. D69, 011104 (2004).

[138] J. S. Kim and K. Lee, Comp. Phys. Comm. 135, 176 (2001).

[139] A. J. Baltz and J. Weneser, Phys. Rev. D35, 528 (1987).

[140] A. J. Baltz and J. Weneser, Phys. Rev. D37, 3364 (1988).

[141] R. Martin, Numerical Methods and Approximations for the Calculation of Solar Neutrino Fluxes in Three Flavours Focused on Results for the Sudbury Neutrino Observatory, Master's thesis, Queen's University, Kingston, Canada (2006). 
[142] M. Blennow et al., Phys. Rev. D69, 073006 (2004).

[143] A. de Gouvêa, A. Friedland, and H. Murayama, Phys. Lett. B490, 125 (2000).

[144] M. Huang, Impact of $N_{\text {prod }}^{\odot}$ and $N_{z e n}^{\oplus}$ on Solar $\nu_{e}$ Survival Probability, SNO Internal Note, MANN-7FNSEX (2008).

[145] G. Tešić, Extraction of Active and Sterile Neutrino Mixing Parameters with the Sudbury Neutrino Observatory, $\mathrm{PhD}$ thesis, Carleton University, Ottawa, Canada (2009).

[146] B. Nickel, Bernie's Adiabatic Method, SNO Internal Note, http://eta.physics.uoguelph.ca/sno/restricted/BGN/ (2008).

[147] J. N. Bahcall, Standard solar model for solar neutrino research, http://www.sns.ias.edu/ jnb (2005).

[148] J. M. Dziewonski and D. L. Anderson, Phys. Earth Planet. Inter. 25, 297 (1981).

[149] D. L. Anderson, Theory of the Earth (Blackwell Scientific Publications, 1989).

[150] J. N. Bahcall and P. I. Krastev, Phys. Rev. C56, 2839 (1997).

[151] C. Giunti and C. W. Kim, Fundamentals of Neutrino Physics and Astrophysics (Oxford University Press, 2007).

[152] Super-Kamiokande, J. Hosaka et al., Phys. Rev. D74, 032002 (2006).

[153] G. L. Fogli et al., Phys. Rev. D66, 053010 (2002).

[154] G. L. Fogli et al., Prog. Part. Nucl. Phys. 57, 742 (2006).

[155] O. Simard, Systematics Definitions for NCD Optics, SNO Internal Note, MANN-6RCN9M (2006).

[156] F. Zhang, Energy response functions for the LETA $D_{2} O$ and salt dataset, SNO Internal Note, MANN-7EDSS6 (2008). 
[157] D. Binosi and L. Theußl, Comp. Phys. Comm. 161, 76 (2004).

[158] SNO Collaboration, P. Harvey et al., QSNO Official Web Page, http://owl.phy.queensu.ca/private/qsno (2009). 